\title{
Leaf Venation Networks
}

\author{
Dissertation \\ for the award of the degree \\ "Doctor rerum naturalium" \\ of the Georg-August-Universität Göttingen
}

within the doctoral program
Physics of Biological and Complex Systems
of the
Georg-August University School of Science (GAUSS)

submitted by

Henrik Michael Ronellenfitsch

from Homburg

Göttingen 2016 


\section{Thesis Committee}

Prof. Eleni Katifori, PhD

Department of Physics and Astronomy, University of Pennsylvania, Philadelphia

Department Physics of Biological Organization, Max Planck Institute for Dynamics and SelfOrganization, Göttingen

Prof. Dr. Annette Zippelius

Institute for Theoretical Physics, Georg-August-Universität Göttingen

Prof. Dr. Jürgen Vollmer

Department Dynamics of Complex Fluids, Max Planck Institute for Dynamics and Self-Organization, Göttingen

\section{Members of the Examination Board}

First Referee: Prof. Eleni Katifori, PhD

Department of Physics and Astronomy, University of Pennsylvania, Philadelphia

Department Physics of Biological Organization, Max Planck Institute for Dynamics and Self-Organization, Göttingen

Second Referee: Prof. Dr. Annette Zippelius

Institute for Theoretical Physics, Georg-August-Universität Göttingen

\section{Further members of the Examination Board}

Prof. Dr. Jürgen Vollmer

Department Dynamics of Complex Fluids, Max Planck Institute for Dynamics and Self-Organization, Göttingen

Prof. Dr. Christoph F. Schmidt

Institute for Theoretical Physics, Georg-August-Universität Göttingen

Prof. Dr. Stefan Luther

Department Biomedical Physics, Max Planck Institute for Dynamics and Self-Organization, Göttingen

Prof. Dr. Reiner Kree

Institute for Theoretical Physics, Georg-August-Universität Göttingen

Date of oral examination: February 15, 2016 
To the memory of my grandfather,

Dipl.-Ing. Werner Arens 


\section{Contents}

I Introduction and Biology of Plant Vasculature 9

1 Introduction 11

1.1 Aim of this thesis . . . . . . . . . . . . . . . . . 12

1.2 Organization . . . . . . . . . . . . . . . 12

2 Biology of Leaves and Leaf Venation 15

2.1 Functional leaf biology . . . . . . . . . . . . . . . . . . 16

2.1 .1 Basic functions . . . . . . . . . . . . . . . . . 16

2.1.2 Water transport and photosynthesis . . . . . . . . . . . 18

2.1.3 Transport of photoassimilates . . . . . . . . . . . 22

2.1.4 Phloem sieve element structure . . . . . . . . . . . 22

2.1 .5 Active transport in the phloem . . . . . . . . . . . . 23

2.1 .6 The leaf in cross section . . . . . . . . . . . . . . . 25

2.1 .7 The shape of a leaf . . . . . . . . . . . . . . . 27

2.1.8 Structure and hydraulics of the leaf venation network . . . . 28

2.1.9 "Design" in nature and the constructal law . . . . . . . . . 32

2.2 Developmental leaf biology . . . . . . . . . . . . . . . . 32

2.2.1 Leaf initiation and the shoot apical meristem . . . . . . . . 32

2.2.2 Vein morphogenesis . . . . . . . . . . . . . . . . 33

2.2.3 Global geometry and folded growth . . . . . . . . . 36

2.2.4 The gymnosperm needle . . . . . . . . . . . . . . 36

2.3 Evolution of leaf venation . . . . . . . . . . . . . . . . 37

2.3.1 Evolution of land plants and leaves . . . . . . . . . . . 37

2.3.2 Evolution of the angiosperm venation network . . . . . . . 38

2.4 Summary . . . . . . . . . . . . . . . . . . 38

II Topological Phenotypes in Leaf Vascular Networks 41

3 Phenotyping Leaf Venation Networks 43

4 Metrics for Planar Networks $\quad 47$

4.1 Graph representation of leaf venation . . . . . . . . . . . . . . 48

4.2 Geometric leaf traits . . . . . . . . . . . . . . . . . . . . 48

4.2 .1 Vein density . . . . . . . . . . . . . . . . . . . . 49 49

4.2 .2 Vein distance . . . . . . . . . . . . . . . . . . . . . . . . . 49 49

4.2 .3 Areole area . . . . . . . . . . . . . . . . . . 49 
4.2 .4 Areole density . . . . . . . . . . . . . . . . . . . 50

4.2 .5 Weighted vein thickness . . . . . . . . . . . . 50

4.3 Topological leaf traits . . . . . . . . . . . . . . . . . . 50

4.3.1 Hierarchical decomposition . . . . . . . . . . . . . . . 50

4.3.2 Quantifying the nesting tree . . . . . . . . . . . . . . 52

4.3.3 Statistical distance between topologies . . . . . . . . . . . . 54

4.3.4 Topological tapering length . . . . . . . . . . . . . . 54

4.3.5 Topological length and nesting number . . . . . . . . . . 55

5 Results 57

5.1 Full leaf networks . . . . . . . . . . . . . . . . . . . . 57

5.1 .1 Principal Component Analysis . . . . . . . . . . . . . . . . 59

5.1 .2 Factor Analysis . . . . . . . . . . . . . . . . . . . . . . . 59

5.1 .3 Topological similarity . . . . . . . . . . . . . . . . 60

5.1 .4 Hierarchical clustering . . . . . . . . . . . . . . . 60

5.2 Leaf network fragments . . . . . . . . . . . . . . . . 61

5.2.1 Linear Discriminant Analysis . . . . . . . . . . . . . . 63

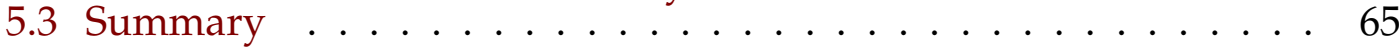

6 Empirical Growth Model $\quad 67$

6.1 The model . . . . . . . . . . . . . . . . . . . . . . . . 68

6.2 Results . . . . . . . . . . . . . . . . . . 70

6.2 .1 Phase diagrams . . . . . . . . . . . . . . . 70

6.2 .2 Comparison with the data set . . . . . . . . . 72

6.3 Summary and discussion . . . . . . . . . . . . . 74

7 Discussion and Outlook $\quad 77$

7.1 Summary and discussion of results . . . . . . . . . . . . . . 77

7.2 Future work . . . . . . . . . . . . . . . . . . 78

7.2.1 Global leaf topology survey . . . . . . . . . . . . . 78

7.2.2 Improving data acquisition techniques . . . . . . . . . . 79

7.2 .3 Systematic study of developmental noise . . . . . . . . . . . 79

7.2 .4 Applications to other fields . . . . . . . . . . . . . . 79

7.2 .5 Improved topological metrics . . . . . . . . . . . . . . 79

III Network Modeling 81

8 Introduction $\quad 83$

8.1 Fitness function models of evolution . . . . . . . . . . . . . . 84

9 Optimal Geometry of Pine Needle Phloem 87

9.1 Introduction . . . . . . . . . . . . . . . . 87

9.2 Mathematical model of sugar flow in needle phloem . . . . . . . 87

9.2.1 Transport equations . . . . . . . . . . . . . . . . . 87

9.2.2 Optimizing the energetic cost of transport . . . . . . . . . . . . 990

9.3 Experimental results . . . . . . . . . . . . . . . . . . 92

9.4 Alternative objective functions . . . . . . . . . . . . . . 93 
9.4.1 Flow rate and volume flow do not explain needle phloem . . 94

9.5 Summary and further work . . . . . . . . . . . . . . . . . . 94

10 Robust Optimal Transport Networks and Auxin Canalization 97

10.1 Robust optimal transport networks . . . . . . . . . . . . . . . . 97

10.1.1 Modeling leaf xylem networks . . . . . . . . . . . . . . 998

10.1.2 Pressure drop minimization . . . . . . . . . . . . . 100

10.1.3 Effects of partial damage on topology . . . . . . . . . . . 102

10.1.4 Increasing resolution . . . . . . . . . . . . . . 105

10.1.5 Summary, challenges, and future work . . . . . . . . . . . 107

10.2 Auxin canalization models of vein morphogenesis . . . . . . . . . . 109

10.2.1 Correlated fluctuations lead to realistic networks . . . . . . . 110

10.2.2 A continuous transition between network topologies . . . . . 112

10.2.3 Summary and future work . . . . . . . . . . . . 113

11Final Summary $\mathbf{1 1 5}$

11.1 Biology of Leaves and Leaf Venation . . . . . . . . . . . . . . . 115

11.2 Topological Phenotypes in Leaf Vascular Networks . . . . . . . . . 115

11.3 Network Modeling . . . . . . . . . . . . . . . . . . . . . . 116

11.3.1 Optimal Geometry of Pine Needle Phloem . . . . . . . . . . 116

11.3.2 Robust Optimal Transport Networks . . . . . . . . . . . . . 116

11.3.3 Auxin Canalization . . . . . . . . . . . . . . . . . . . . 117

11.4 Have we achieved our aims? . . . . . . . . . . . . . . . . . . . 117

$\begin{array}{ll}\text { Appendix } & 119\end{array}$

$\begin{array}{ll}\text { A Statistical Techniques for Phenotyping } & 121\end{array}$

A.1 Statistics . . . . . . . . . . . . . . . . . . . . . 121

A.1.1 The Gaussian distribution . . . . . . . . . . . . . . 122

A.1.2 Statistical tests . . . . . . . . . . . . . . . . . . 122

A.1.3 Validation techniques . . . . . . . . . . . . . . . . . 124

A.2 Information theory and statistical distances . . . . . . . . . . . . 125

A.2.1 Entropy . . . . . . . . . . . . . . . . . 125

A.2.2 Distance measures . . . . . . . . . . . . . . . . 126

A.3 Data and Covariance . . . . . . . . . . . . . . . . . . . . . 129

A.4 Covariance analysis . . . . . . . . . . . . . . . . . . . . . 130

A.4.1 Principal Component Analysis . . . . . . . . . . . . . . . 130

A.4.2 Factor Analysis . . . . . . . . . . . . . . . . . . 131

A.5 Clustering algorithms . . . . . . . . . . . . . . . . . . 132

A.5.1 k-means . . . . . . . . . . . . . . . . . . 133

A.5.2 Gaussian Mixing Models . . . . . . . . . . . . . . . . . . . . 133

A.5.3 Hierarchical clustering . . . . . . . . . . . . . . . . . 134

A.6 Classification algorithms . . . . . . . . . . . . . . . . . 135

A.6.1 Linear and Quadratic Discriminant Analysis . . . . . . . . 135

$\begin{array}{ll}\text { B The Leaf Data Set } & 137\end{array}$ 
B.1 The data set . . . . . . . . . . . . . . . . . . . . . . . . . 137

B.2 Vectorization . . . . . . . . . . . . . . . . . . 138

B.3 Automatic pre-processing of the leaf graphs . . . . . . . . . . 139

B.3.1 Removal of nonplanar artifacts . . . . . . . . . . . . . 140

B.3.2 Removal of collinear edges . . . . . . . . . . . . . . . . . 140

B.3.3 Cycle pruning . . . . . . . . . . . . . . . . . . . . 140

B.3.4 Choice of connected component . . . . . . . . . . . . . . 141

B.4 Constructing the fundamental cycle basis. . . . . . . . . . . . . 141

C Nesting Number Analytics $\quad 143$

D Linear Flow Networks 147

D.1 Linear algebra of graphs . . . . . . . . . . . . . . . . . 147

D.1.1 The node vector space . . . . . . . . . . . . . . . . . . 148

D.1.2 The edge vector space . . . . . . . . . . . . . . . . . . . . 149

D.1.3 The cycle vector space . . . . . . . . . . . . . . . . . . . . 149

D.1.4 Algebraic topology of graphs . . . . . . . . . . . . . 150

D.2 Linear flow networks . . . . . . . . . . . . . . . . . . . 151

D.3 Edge perturbations in linear flow networks . . . . . . . . . 153

D.3.1 Power dissipation . . . . . . . . . . . . . . . . . . . . 154

D.3.2 Cycle flows and decay of perturbations in 2D . . . . . . 155

D.3.3 Decay of perturbations in the continuum approximation . . . 155

D.4 Summary . . . . . . . . . . . . . . . . . . . . . 161 

Part I

\section{Introduction and Biology of Plant Vasculature}





\section{Introduction}

Das Blatt, auf dem die Raupe lebt, ist für sie eine Welt, ein unendlicher Raum.

(Ludwig Feuerbach)

As Hallé states in [1], "Humans have always preferred animals to plants, no matter what time and place". The reason is simple: animals tend to exhibit motion immediately exciting our mind, whereas plants, seemingly sessile, barely even appear alive. ${ }^{1}$

Clearly, plants are very different from animals. Their mode of being proceeds on timescales too large to be immediately recognized by us. Their form, even though ubiquitous in daily life, is almost alien to us: a strange, fractal-like body, each part seemingly consisting of a small copy of the whole. Yet, we are completely dependent on them as they alone can perform photosynthesis, converting energy from sunlight into a form usable by us. Despite their strangeness (that we may not even notice because of their omnipresence!) and the fact that we are entirely dependent on them for survival, we often neglect them save for the admiration for a pretty flower.

Plants deserve recognition. From the overall space-filling, fractal growth of tree branches to the phyllotactic patterns of individual leaves and leaflets, their form exhibits remarkable mathematical symmetries and a degree of strict order and regularity that is not often found in animals. Because they lack centralized control (there is no equivalent of the mammalian central nervous system or a central fluid pump such as a heart), a plant's life is much more governed by local, decentralized, often passive, self-organized processes. These include growth, transport of fluids, induction of flowering, and many more.

In this thesis, we study the leaf, together with the roots arguably the most crucial organ of modern vascular plants. Its function is to perform photosynthesis, thus keeping the rest of the plant body alive. The forces of evolution have shaped the leaf into a remarkably optimized machine whose transport system delivers vital water throughout the leaf blade and transports away sugars, chemically preserving the sun's energy.

In modern vascular plants, the leaf venation system arises through a selforganized morphogenetic process, resulting in a dense, highly reticulate planar network structure that is optimized to be efficient and resilient to external damage and fluctuations. This thesis is an attempt to further our knowledge of the structure, topology, and morphogenesis of the leaf venation network which is, despite its ubiquity, poorly understood.

\footnotetext{
${ }^{1}$ In some languages that possess a grammatical category of animacy, or aliveness, plants are considered inanimate.
} 


\subsection{Aim of this thesis}

Understanding the leaf venation network is a multifaceted endeavor that requires tools from several fields of study. This thesis addresses a broad range of questions, all guided by a central theme: "How does the geometry and topology of leaf venation depend on its function, development, and evolution?". The aim of this thesis is to make a small contribution towards answering this immensely broad question and naturally, not every question can be answered. We attempt a multi-pronged approach that we hope can serve as a first stepping stone to more integrative studies in the future. To begin to attack it, it is necessary to break the question down into smaller pieces that can be addressed individually.

We ask, broadly, "What is the connection between the geometry and the topology of leaf networks?", and "What is the connection between the physics of evolution and development of leaf networks?". The first question is attacked in Part II, the second in Part III.

Still, these questions are too broad to be answered in a single dissertation. Nonetheless, we hope that this work may provide a modest contribution to the science of plant vasculature from a physicist's perspective. In particular, we hope that further studies will be facilitated through the software package nest ing that grew out of the work for Part II and that is freely available for anyone to use and measure the topological structure of leaf venation networks and even more general planar weighted graphs. The software package available at http://www.github.com/hronellenfitsch/nesting.

\subsection{Organization}

The rest of this dissertation is organized in three parts and an appendix. In Part I, we review the basic biology of plant leaf vasculature, including function, development, and evolution. We also review leaf and plant hydraulics.

In Part II, we investigate the topological organization of leaf vascular networks, providing the first ever study of the hierarchical nesting of loops in a large data set of real leaves and showing that topology appears to be a new, previously undescribed phenotypic trait of the leaf. We present a simple, empirical model that explains much of the observed variation in topology and demonstrate the usefulness of this new trait.

Finally, in Part III, we study the physics of leaf venation networks more directly by investigating models of its evolution and development. We show that very simple models of development can produce the type of network topology predicted by evolutionary models that is remarkably close to real leaves. 


\section{Acknowledgments}

This thesis would have been impossible without the help of many persons. First of all, I wish to thank Eleni Katifori for providing the opportunity to conduct the research projects contained here and for many stimulating discussions and continued support. Additionally, many enlightening discussions were had with fellow group members Jana Lasser, Johannes Gräwer, and Jonathan E. Dawson. I thank Johannes Gräwer, Jana Lasser, and Eleni Katifori for thoroughly reading the thesis manuscript and many helpful comments. For the results in Part II, Douglas C. Daly provided access to the cleared, stained, and plasticized leaf samples that were scanned by Eleni Katifori. Jana Lasser wrote the software package to vectorize the digitized samples. The work described in Chapter 9 was done in collaboration with Kåre H. Jensen, Alexander Schulz, Johannes Liesche, and N. Michele Holbrook, who performed measurements and provided invaluable physical and biological insight. The cycle flow idea used in Chapter D of the appendix was developed by Dirk Witthaut. Marc Timme also gave valuable insight here. I also wish to express my immense gratitude for the institute secretaries Barbara Kutz and Monika Teuteberg, and the IT administrators Denny Fliegner and Yorck-Fabian Beensen who kept things running smoothly in the background.

Finally, I wish to thank my family and friends who continually supported me through my studies.

\section{License}

The content of this thesis is licensed under the terms of the Creative Commons Attribution-ShareAlike license (CC BY-SA 4.0) with the exception of figures and images that were reprinted with permission of the original copyright holders. The full license text can be found at

https://creativecommons.org/licenses/by-sa/4.0/.

In addition, this thesis contains attributed reproductions and adaptations of works licensed under the CC BY-SA 3.0/4.0 and the CC BY licenses. The full license texts for the original images can be found at

https://creativecommons.org/licenses/by-sa/3.0/

and

https://creativecommons.org/licenses/by/3.0/.

Image source links are provided in the List of Figures. 



\section{Biology of Leaves and Leaf Venation}

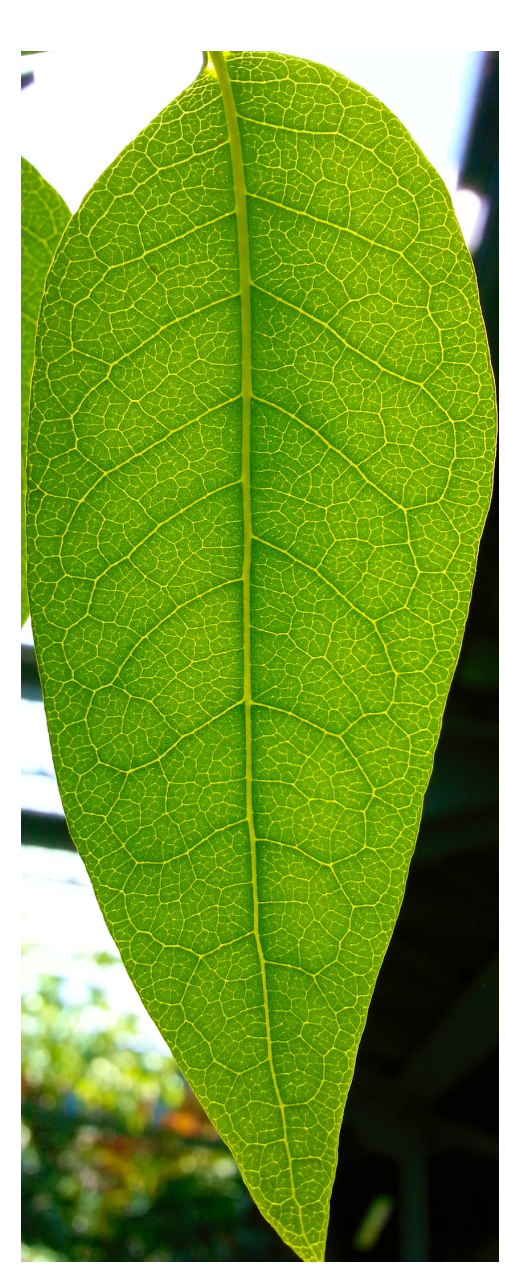

FIGURE 2.1: Leaf blade of a dicot. The highly complex venation network is clearly visible.

In this section, we give an overview of the biology and biomechanics of our main object of study, the angiosperm dicotyledonous (dicot) plant leaf. We discuss the leaf's basic biological structure, function, evolution and development, as well as its relation to the rest of the plant body.

The leaf (see Figure 2.1) is an extraordinarily complex plant organ that in addition shows great variability and diversity of form amongst the angiosperms. Still, even given this staggering diversity, all leaves follow a basic recipe of form and function which we will try to present here. The strategy will be to in a way look at the leaf from various different "angles", each time uncovering new physical and biological properties.

In Section 2.1, we first focus on the functional aspects of the fully matured leaf. We describe its basic functions, and how its form allows it to accomplish them in an efficient manner. We then proceed to look at a leaf in cross section, observing in detail the various sub-organs inside, in particular focusing on form and function of the venation system. Finally, we look at the leaf from the outside, exploring the various possible shapes that occur and how they are related to function.

In Section 2.2, we then proceed to describe the mechanism used by the plant to produce new leaves from the shoot apical meristems, hosts of totipotent stem cells. We then pay particular attention to vein morphogenesis and the current hypotheses and models that are used to describe it. Finally, we give a short account of conifer needle development even though our main focus lies on the angiosperms, not the gymnosperms. This is done because conifers provide a one-dimensional model of venation in contrast to the more complicated two-dimensional venation found in angiosperm dicots.

Last, in Section 2.3, we give a short account of the evolution of leaves and leaf venation, with a particular focus on the development of the angiosperms and the reasons behind their remarkable evolutionary success. 
The content of this chapter is well-known biology and biomechanics, and draws from many good textbooks. For the basic functional biology and development we found [2] particularly helpful and clear. An excellent introductory book about leaf biomechanics is [3]. Wherever the content presented here goes beyond these introductory texts, we will refer to the relevant scholarly articles. In order to make this introduction more palatable to the physically inclined reader, we will intersperse the biological facts with the relevant physics describing the particular biological system in question.

\subsection{Functional leaf biology}

Leaves are ubiquitous in the world around us. This is no accident, as together with the roots, they are the most important organ of all living higher plants. The leaf is the result of hundreds of millions of years of evolutionary adaptation, and as such it showcases several crucial functions that assure survival for both the tiniest meadow flowers and the tallest trees. The most important function is fixation of solar energy by photosynthesis. In this section, we will first follow [3] in our description of the basic functions, and later move on to the leaf vasculature in more detail, since this is what will be of interest in the rest of this thesis. We should keep in mind though that this reductionist approach ignores many of the subtleties of leaf biology. The leaf is a highly intricate, interconnected machine, and discarding parts of it we will necessarily lose some aspects that may be crucial. Nevertheless, this is the ap-

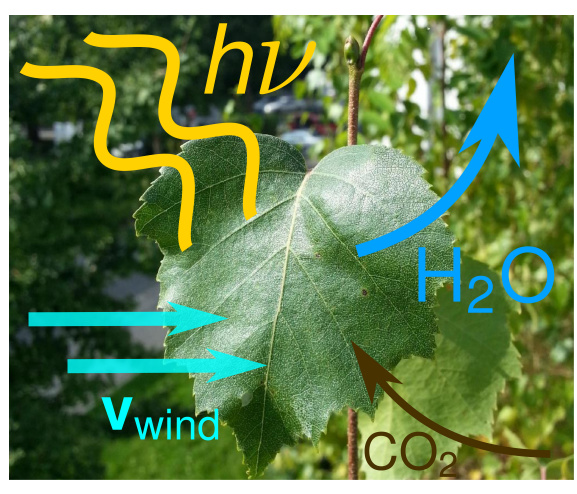

FIGURE 2.2: Schematic diagram showing the main leaf interactions with the environment. Sunlight (yellow waves) hits the leaf blade, providing energy for photosynthesis as well as heating up the leaf blade. Wind (light blue arrow) leads to drag as well as convective cooling. Water (dark blue arrow) evaporates from the leaf blade during gas exchange with carbon dioxide (brown arrow).

\subsubsection{Basic functions}

In games of strategy such as Chess or Go, the prime property of a good move is that is achieves more than one goal at a time given a set of highly challenging constraints. Evolution has had billions of years to perfect its moves, and indeed, the dicot leaf is an excellent example of a multifaceted strategy. Four prime goals for the leaf to achieve are identified in [3]. They are all based on how the leaf needs to interact with its immediate environment, which typically contains sunlight providing the energy for photosynthesis as well as heating up the leaf, and wind that provides convective cooling as well as drag. The leaf constantly exchanges gases $\left(\mathrm{O}_{2}\right.$ and $\left.\mathrm{CO}_{2}\right)$ with the environment, losing water in the process (see Figure 2.2). These basic functions can be summarized as [3]: 
1. Intercept light. Performing photosynthesis is the main function of the leaf. The leaf needs to provide the appropriate chemical environment for this life-giving reaction that converts the energy of the sun into sugars (photoassimilates). Sugars which serve as the basis for all other metabolic processes in the plant body, and indirectly those of all animals on the planet. The leaf must be such that it maximizes the amount of sunlight it can catch while at the same time being mechanically rigid enough to keep its shape.

2. Get water up. Photosynthesis requires copious amounts of water, much more than what could be absorbed from the atmosphere. Thus, the plant relies on its root system together with an intricate network of vascular tissue (the xylem) to provide the leaf with water from the ground. Inside the leaf itself, there is a complex network of interconnected veins that provide every part of the leaf with sufficient water. The method employed to get the water moving is entirely passive, relying on negative pressure due to evaporation at the leaf surface.

3. Do not overheat. Leaves are exposed to the sun, a necessity for photosynthesis to occur. Yet, they cannot use all of the energy contained in sunlight efficiently, and therefore they heat up. So much so that the leaf needs to deploy a host of additional measures to keep its temperature in an acceptable range and prevent death from overheating.

4. Do not be too draggy. All larger plants and especially trees are exposed to wind. Since leaves are necessarily numerous and almost flat, they are subject to a considerable amount of drag - possibly a dangerous amount, as wind forces may be strong enough to topple a tree. In order to minimize drag, the leaf's mechanical properties allow it to bend, curl and cluster in high winds, reducing drag.

Because this thesis is about physical properties of the vascular network in leaves, we will mostly ignore basic functions 3 and 4 in the remainder of the text. However, in addition to the functions from [3], we propose another one.

*5. Get photoassimilates out. As a living organ, the chemical processes in the leaf itself consume some of the energy captured from sunlight. However, almost all other plant organs such as shoots, roots, flowers and fruits are unable to photosynthesize on their own and therefore energy must be transported to them. Thus, the leaf needs to contain an additional vascular system (the phloem) whose function it is to remove photoassimilates (sugars) and effectively "feed" the rest of the organism.

The rest of this section will be concerned with the structure of the leaf and its vascular system, showing the principles that nature employs to facilitate efficient water uptake and delivery and efficient translocation of photoassimilates and thus efficient photosynthesis. 


\subsubsection{Water transport and photosynthesis}

Water is arguably the most crucial substance that plants need, and therefore evolution has provided them with a highly complex system of water uptake, transport, and delivery, surpassed in complexity maybe only by the vascular system of animals, but certainly not surpassed in ingeniousness of mechanism. This section discusses the interplay between water transport and photosynthesis. It is based mainly on the expositions in $[2,4,5]$ and [6].

\subsubsection{Gas exchange and the evolution of photosynthesis}

Even though water is one of the most abundant substances on the planet, plants struggle to obtain a sufficient amount of it. In fact, they retain only about $5 \%$ of all water taken up by the roots, the rest being lost to the atmosphere through a process called transpiration. The reason lies in photosynthesis. The basic net chemical reaction is [7]

$$
\mathrm{CO}_{2}+2 \mathrm{H}_{2} \mathrm{X}+\text { light energy } \longrightarrow\left[\mathrm{CH}_{2} \mathrm{O}\right]+2 \mathrm{X}+\mathrm{H}_{2} \mathrm{O},
$$

where $\mathrm{X}$ is an oxidizing agent (e.g., $\mathrm{O}$ ) and $\left[\mathrm{CH}_{2} \mathrm{O}\right]$ represents a sugar. The carbon dioxide necessary for this reaction to occur comes from the surrounding air, thus the leaf needs to open up in order to facilitate intake of $\mathrm{CO}_{2}$. This is achieved through the stomata (see Figure 2.3), small pores in the leaves the openings of which the plant is able to control. Opened stomata allow gas molecules to freely diffuse between the inside of the leaf and the surrounding air, leading to the $\mathrm{CO}_{2}$ assimilation rate [8]

$$
A=g_{\mathrm{CO}_{2}}\left(\mathrm{C}_{\text {air }}-\mathrm{C}_{\text {leaf }}\right),
$$

where $C_{\text {airleaf }}$ are the effective $\mathrm{CO}_{2}$ pressures outside and inside of the leaf and $g_{\mathrm{CO}_{2}}$ is the effective diffusivity for $\mathrm{CO}_{2}$.

Because water is abundant inside the leaf but $\mathrm{CO}_{2}$ is rare in the atmosphere, a staggering amount of water is lost in this process of gas exchange. Indeed, for one molecule of carbon dioxide gained, 400 molecules of water are lost [4]. This is the ratio between the $\mathrm{CO}_{2}$

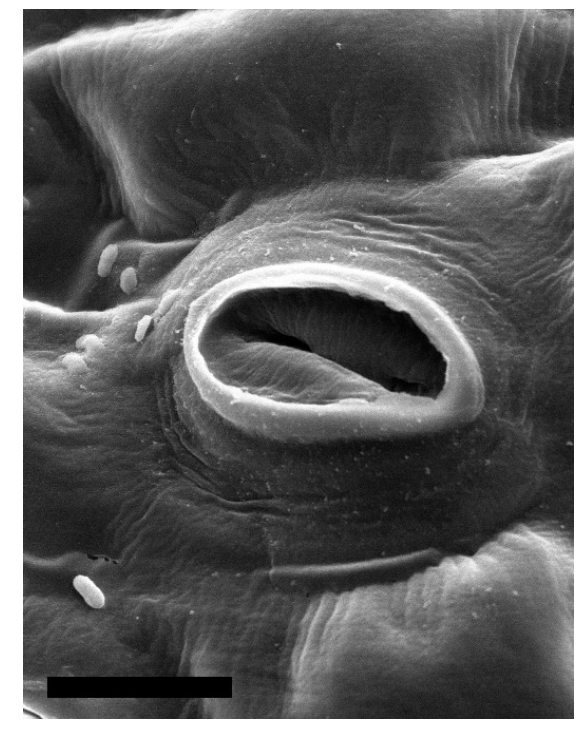

FIGURE 2.3: Electron micrograph of a single stoma on a tomato leaf surface. The stoma is partially opened to facilitate gas exchange. The scale bar is $10 \mu \mathrm{m}$. (Image by Louisa Howard, Dartmouth College, public domain.) assimilation rate $A$ and the water transpiration rate $E[6]$,

$$
\frac{A}{E}=\frac{1}{1.6} \frac{C_{\text {air }}-C_{\text {leaf }}}{p_{\text {air }}-p_{\text {leaf }}}
$$


where $p_{\text {air,leaf }}$ is water vapor pressure outside and inside of the leaf and 1/1.6 is the ratio of effective diffusivities for $\mathrm{CO}_{2}$ and water vapor.

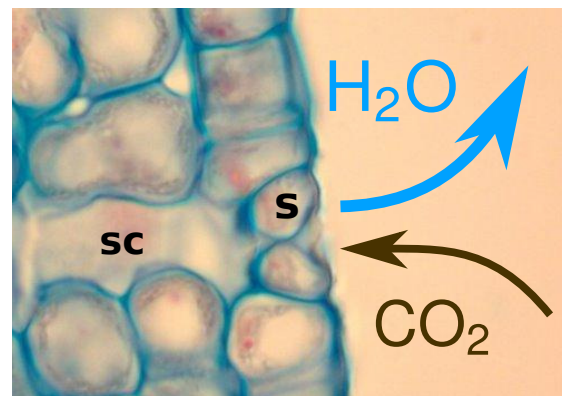

FIGURE 2.4: Gas exchange through a stoma. Water evaporates inside the stomatal cavity (sc) and diffuses through the stoma (s) It is exchanged for carbon dioxide which diffuses in from the outside. ("Dwars doorgesneden huidmondje in het blad van een dicotyle" by Yvan Lindekens, CC BYSA 3.0 / cropped and annotated.)

One possible reason for this remarkable squandering of precious water is the fact that when photosynthesis first evolved roughly 2.9 billion years ago in methanotrophic archaea, it did so in the ocean, where access to water is not a concern [9]. The crucial protein necessary for carbon fixation that evolved at that time, RuBisCO I, is still used by all modern plants, algae, and cyanobacteria [9-11], making it the most abundant protein on the planet [12]. Its catalytic efficiency is also remarkably low [12], showing that evolution appears to favor the "never change a running system" approach to engineering. For modern dicots, this leads to a fundamental dilemma: Open your stomata and risk dehydration, or close them and risk starvation.

\subsubsection{Uptake at the roots}

Water is taken up from the soil through the roots, a complex branching network of individual strands often covered by fine hairs that increase surface area and thus the ability to absorb water. They have the ability to grow away from dry areas (hydrotropism) and form huge exploratory networks in order to find wet soil deep underground. The exact mechanism of this hydrosensing is not known, but the root cap is likely responsible.

After being absorbed by the root, water travels either through the cell walls (the apoplast) or inside the cells (the symplast) until it reaches the specialized transport tissue responsible for efficient movement through the plant [13].

\subsubsection{Transport through the plant: cohesion-tension theory}

Specialized water transport cells (the xylem vessels) are responsible for keeping an intact column of water between the root and the leaf, where finally, transpiration happens. The mechanism thought to underlie this transport is called Cohesion-Tension (CT) theory $[13,14]$. The basic logic behind CT theory can be summarized as follows.

- There is a continuous column of water starting at the plant roots and extending up into the leaves.

- Inside the stomatal cavities, water evaporates, thus creating tension on the water column. This tension is responsible for pulling up more water. 
- Tension on the water column is counteracted by cohesive forces (hydrogen bonds) in water.

- The water inside the xylem is in a metastable state, effectively at pressures below vacuum, and thus prone to cavitation.

We note that the entire mechanism transporting water inside the plant as described by CT theory is passive, meaning that there are no metabolic pumps doing any sort of work to move liquid. The forces responsible for the motion of water are generated by evaporation alone. ${ }^{1}$

Although very successful in describing the flow of liquid in plants, CT theory, established over 100 years ago [16] is not free from controversy, in part because of a perceived "improbability" of water under high tension $[13,14,17]$. However, several physical arguments exist that render the idea plausible after all. The simplest one estimates the cohesion pressure by the energy stored in the hydrogen bonds to about $1400 \mathrm{MPa}$. This is unrealistically high, and more sophisticated estimates find values between $50 \mathrm{MPa}$ and $300 \mathrm{MPa}$ [13], sufficient to offset the tensions necessary to transport water in a tall tree. Typical values of tensile pressure in plants tend to be around $-2 \mathrm{MPa}$ (in crop plants) with the record at around $-12 \mathrm{MPa}$ (in plants adapted to highly arid climate) [3]. Other critics invoke spontaneous nucleation of gas bubbles as a weakness of the theory, but arguments against this exist as well [13], and in fact, evolution appears to have endowed plants with mechanisms to recover from such gas embolisms [18, 19]. An additional argument towards the validity of CT theory consists of the construction of microfluidic "synthetic trees," modeling the hydraulic properties of a real tree. Such models have been built and successfully used to demonstrate the plausibility of CT theory [20].

The physical model underlying CT theory is similar to Darcy's law from the theory of porous
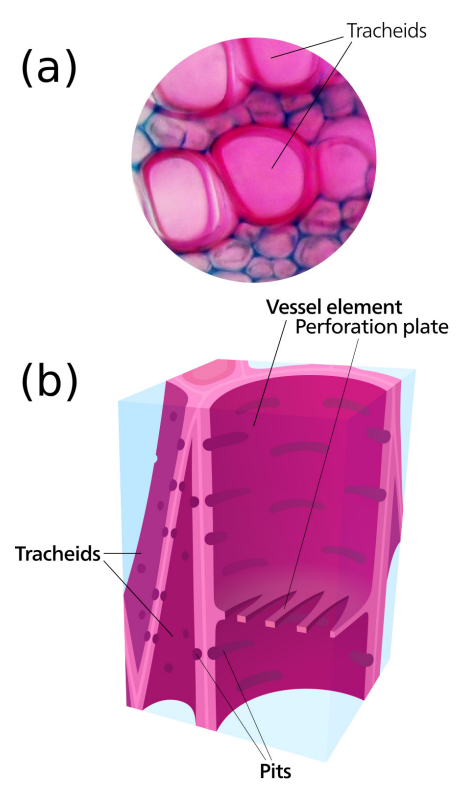

FIGURE 2.5: Anatomy of the xylem. (a) Cross-sectional micrograph of xylem tissue showing tracheid cells. (b) Sketch of xylem cell anatomy. Vessels are subdivided by perforation plates and connected to adjacent tracheids through pits in the cell wall. ("Diagram of xylem tissue in plants" by Kelvin Song, CC BYSA 3.0 / rearranged and simplified). flow [21] as well as the law of Hagen-Poiseuille. Volume flow of water through a small xylem section is described by

$$
F=-K \frac{d p}{d x}
$$

\footnotetext{
${ }^{1}$ There is some "pushing" of water from the roots, but the effect is only relevant in herbs and shrubs [15] and mostly negligible in trees.
} 
where $F$ is volume flow, $p$ is hydrostatic pressure, and $K$ is xylem hydraulic conductivity. In the case of a cylindrical cell of radius $r$, the conductivity works out to be

$$
K=\frac{\pi r^{4}}{8 \eta}
$$

where $\eta$ is the dynamic viscosity of water. This is the law of Hagen-Poiseuille. The dependence on the fourth power of the radius means that the contribution of small transport tissue can be negligible.

This law also resembles Ohm's law of electrical circuits

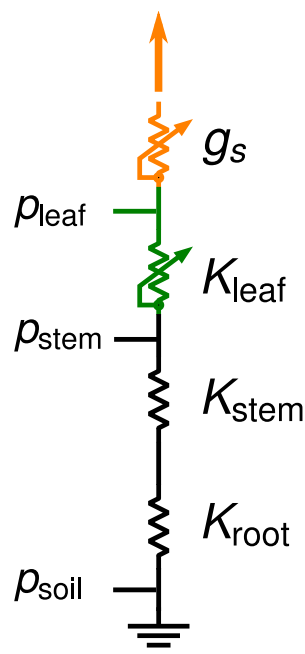

FIGURE 2.6: Hydraulic resistor model for a tree. The hydraulic conductance $K$ is partitioned into components for root, stem, and leaf. Additionally, the stomatal conductance $g_{s}$ is shown. Both $K_{\text {leaf }}$ and $g_{s}$ are variable because their values strongly depend on whether and how many stomata are opened or not (Simplified after [5]). and indeed, it is possible to model water flow in plants and microfluidic devices in terms of networks of hydraulic resistors (see Figure 2.6 and [22]). It is important to note that equations (2.4) and (2.5) are valid only for laminar flow. Inside plants, the Reynolds number is of the order $R e \approx 0.01$ to 0.1 , far below the critical regime for turbulence [23]. Typical flow speeds are $20-40 \mathrm{~cm} \mathrm{~h}^{-1}$ in conifers and $5 \mathrm{~m} \mathrm{~h}^{-1}$ in broad-leaved trees [24].

The xylem tissue consists of two separate kinds of conducting cells, tracheids and vessels. Tracheids are smaller in length and diameter and taper off at the end. Long vessels consist of stacks of individual vessel elements. Vessel diameters range between 20 and $100 \mu \mathrm{m}$, vessel lengths between 2 and $20 \mathrm{~cm}$ [25]. Immediately after maturation, xylem cells undergo apoptosis, shedding their nucleus and other internal cell organelles. What is left over is a dead, hollow cell that nonetheless is only now ready to fulfill its function as both carrier of water and provider of mechanical stability through strong lignin fibers in its walls.

The vessels themselves are connected by overlapping their ends, forming networks. Once liquid has reached the end of one vessel, it moves to the next one through membranes in openings along the lateral cell walls called pits. The pit membranes allow water to pass but act as a stop to air bubbles, preventing the spreading of cavitation through the entire plant. Thus, damage to the transport system is limited. Across species, the structure of the pit walls varies considerably [26]. In order to create a single vessel tube, the cell walls between neighboring consecutive vessel elements need to be partially dissolved so as to permit liquid to flow. This results in the appearance of perforation plates inside xylem vessels. Several different morphological types of perforation plates exist. Hypotheses as to why perforation plates exist at all (minimizing flow resistance would mean doing away with them completely) invoke increased resistance against embolisms both 
during freeze-thaw cycles and after cavitation. Instead of one large air bubble, perforation plates would create several smaller bubbles that dissolve more easily. Additionally, air is trapped by the perforation plates, preventing several small embolisms from coalescing in a fatal manner [23]. Figure 2.5 contains both a representative micrograph of xylem tracheids as well as a sketch of the vessel anatomy described.

Finally, water enters the leaf through the petiole where it is distributed by a network of veins and eventually evaporates or is used up in the photosynthetic pathway. The venation network inside the leaf itself is complex enough to warrant its own discussion, and will be presented in detail in Section 2.1.6 and Section 2.1.7.

\subsubsection{Transport of photoassimilates}

All organs of the plant need to be continuously supplied with chemical energy in the form of sugar molecules in order to perform their metabolic functions. However, not all plant organs possess the necessary cellular apparatus (chloroplasts) to photosynthesize, and some lack exposure to the sun entirely (the roots). Thus, it is necessary to transport energy, stored chemically in sugar molecules (photoassimilates), from the sites of photosynthesis (the leaves) to sites of consumption and growth such as roots, shoots and fruits (the heterotrophic organs, in contrast to the autotrophic leaves). Transport of photoassimilates is taken over by the phloem, a type of vascular tissue distinct from the xylem but still closely related. Aside from its primary function, the phloem is also responsible for transporting defensive compounds and information signals in the form of phytohormones throughout the plant body, rendering it comparable to a sort of combination of animal vascular, lymphatic, and nervous system. In this section we describe the basic mechanism of phloem translocation in a plant. The exposition is based on the reviews $[27,28]$.

\subsubsection{Phloem sieve element structure}

The phloem cells which are responsible for transport of sugars are called sieve cells or sieve elements. Unlike the xylem, they are alive even though they shed their nucleus and most of the internal cell organelles during maturation. Because they do not possess the machinery necessary for most of standard cell metabolism, they rely on associated companion cells (or in conifers, Strasburger cells) to keep them alive. Like xylem vessels, the sieve elements are elongated and optimized for transport of sap and form long stacks. Single sieve cells have typical diameters up to $20 \mu \mathrm{m}$ with records around $50 \mu \mathrm{m}$ [29]. The length/diameter ratio tends to be between 10 and 100 [29].

In order to facilitate flow, the cell walls between adjacent sieve elements are perforated, similar to the perforation plates of the xylem. These walls are called sieve plates, and some work has been done to estimate their effect on hydraulics $[30,31]$. Figure 2.7 shows a micrograph of phloem tissue as well as an 
illustration of the cell anatomy described.

The sap inside the sieve elements is rich in energy, containing 10-30\% sugar by weight (mainly sucrose) $[28,30]$, as well as various other substances such as flower-inducing phytohormones, and is therefore highly valuable to the plant. It has been suggested that a certain protein (P-protein, or proteinacious sieve tube slime) is used by the plant in response to damage in order to plug the sieve pores and thus prevent loss of sap, but the idea remains controversial $[33,34]$. It is interesting to note the difficulty of sampling phloem sap due to the plant's defense mechanisms. Certain aphids have evolved a highly specialized organ (the stylus) that is able to penetrate the plant epidermis and directly tap into the phloem. These animals provide the most efficient way to obtain phloem sap for analysis, better than any other method [35].

Contact of sieve cells with life-preserving, ontogenetically related companion cells is made through extensive sets of intercellular pores called plasmodesmata. In contrast to the sieve cells, the companion cells contain a large number of ribosomes and mitochondria, rendering them highly metabolically active and allowing them to provide the sieve cell with the necessary enzymes, membrane proteins, and metabolites to ensure survival [28]. In addition to this function, the companion cells are often responsible for loading and unloading of photoassimilates into and out of the sieve cells [36]. This is the crucial step for the functioning of the Münch mechanism driving active long-range translocation described below.

\subsubsection{Active transport in the phloem}

Unlike flow of water in the xylem which is passively driven by evaporation in the stomatal cavities, phloem translocation is actively driven by osmotic pressure gradients along the whole length of the phloem sieve tube network. The mechanism was first proposed by Münch in the 1920s [37]. Sugars produced in the leaf are loaded into phloem sieve elements by companion cells (depending on species using one of various available mechanisms). This generates an osmotic concentration gradient with respect to the surrounding tissue, driving water into the sieve elements. At the sink tissue, sugars are unloaded from the phloem and carried into the surrounding cells. Again, this leads to an osmotic gradient driving water out of the phloem. In total, the result is a net bulk flow of water

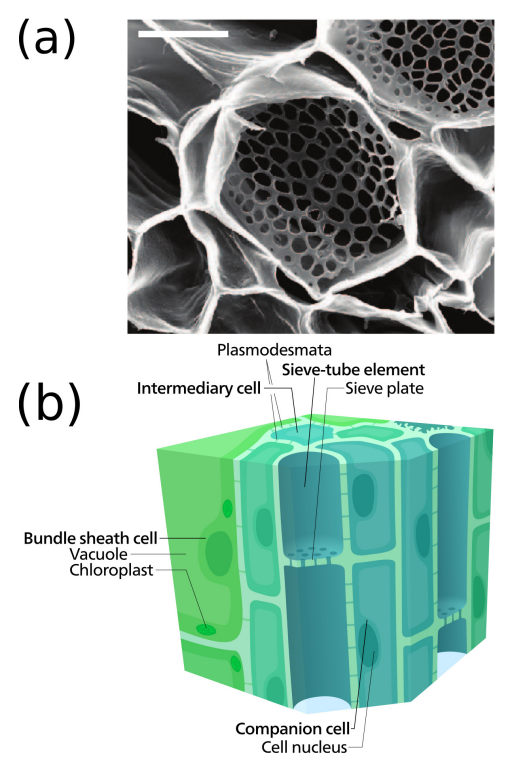

FIGURE 2.7: Phloem anatomy. (a) SEM image of phloem cell showing sieve plates. The scale bar is $10 \mu \mathrm{m}$. (b) Sketch of phloem cell anatomy. Sieve tubes are separated by sieve plates and connected to companion intermediary cells into the sieve tubecompanion cell complex. ("Diagram of phloem tissue in plants" by Kelvin Song, CC BY-SA 3.0 / cropped and simplified). 
from source to sink tissues, driven by an osmotic gradient [30]. Photoassimilates are carried advectively by this bulk flow (the Péclet number has been estimated to $P e \approx 10^{5}$ [38]; diffusion of solutes is irrelevant). Typical bulk flow speeds are in the range of $1 \mathrm{~m} \mathrm{~h}^{-1}$ for angiosperms and $0.1 \mathrm{~m} \mathrm{~h}^{-1}$ for conifers [30].

Translocation of solutes in a whole plant can be modeled using a hydraulic resistor model (much like the xylem, see Section 2.1.2.3 and Figure 2.6) with total hydraulic resistance [30, 39]

$$
\begin{aligned}
R_{\text {tot }} & =1 / K_{\text {tot }} \\
& =R_{\text {source }}+R_{\text {stem }}+R_{\text {sink }} .
\end{aligned}
$$

Water must traverse a semipermeable membrane in the sink tissue. Assuming a cylindrical sieve tube of radius $r$ and length $\ell$ with lateral membrane permeability $L_{p}$, the hydraulic resistance is $R_{\text {source }}=1 /\left(2 \pi r \ell L_{p}\right)$. Similarly, in the sink tissue of length $s$ we have $R_{\text {sink }}=1 /\left(2 \pi s \ell L_{p}\right)$. In the stem of height $h$, the standard Hagen-Poiseuille formula can be used with $R_{\text {stem }}=8 \eta h /\left(\pi r^{4}\right)$. This yields water velocity $u$ in the stem as

$$
u=\frac{1}{\pi r^{3}} \frac{\Delta p}{R_{\text {source }}+R_{\text {stem }}}=\frac{2 r^{2} L_{p} \ell}{r^{3}+16 \eta L_{p} \ell h} \Delta p .
$$

Here $\Delta p$ is the pressure drop along the length of the tree. Surprisingly, this number does not scale with height and can be estimated to a constant $\Delta p \approx$ $10 \mathrm{MPa}[40,41]$. The sugar transport rate is then $j=c u$, where $c$ is sugar concentration. This model allows for several interesting estimates on maximum tree height and leaf size using transport rate optimization arguments [39]. It can also be used to derive a scaling relation between sieve element radius and plant height that has been empirically verified [30,39,42]. A more refined model is able to predict concentration profiles and translocation speeds analytically on the whole-tree level $[43,44]$. In reality, phloem tubes do not occur in isolation but instead are part of a vascular bundle consisting of several similarly sized tubes. Still, continuous models are possible [45].

So far, we have neglected the details of phloem loading and unloading even though they are the crucial step that makes bulk flow possible. In the source tissue, photoassimilates need to be transported against the concentration gradient into the phloem. In most plants, active accumulation in the region close to a sieve element-companion cell complex occurs. Several different mechanisms have been proposed, and different plants use different means to accrue sugars [28, 36]. Apoplastic loaders release photoassimilates into the water-filled space inside the cell wall (the apoplast) from where it is taken up by the adjacent cells. For this mechanism, active transporter proteins are necessary to pump sucrose out of the cytoplasm.

Symplastic loaders utilize pores (plasmodesmata) between neighboring cells to move sugars between them. Typically, plants that utilize the symplastic route 
employ raffinose or higher polymers of sucrose. In contrast to apoplastic loaders, symplastic loaders possess very large numbers of plasmodesmata. The polymer trap mechanism allows these plants to accrue sugar against the concentration gradient through the symplast. Sucrose diffuses freely into intermediary cells where it is enzymatically polymerized into raffinose or higher sugars. These are too large to diffuse back but small enough to diffuse further into the sieve tube-companion cell complex. ${ }^{2}$

Finally, some plants such as Salix (willow) employ phloem that is directly linked to the surrounding tissue, such that here, diffusion along the concentration gradient occurs. The motor of "phloem loading" in this case is simply the process that transports sucrose out of the photosynthesizing chloroplasts [28, 46].

In summary, the phloem, together with its associated companion cells as well as the intermediary cells partly responsible for loading, appears to be a remarkably complex system, much more than the comparatively simple xylem. Instead of passive, dead tissue in the xylem, the phloem is alive, active and thus more difficult to study. Evolution has brought forth several different, complicated mechanisms for the accumulation of sugars in the transport tissue. In the case of gymnosperms, the whole process is still even more poorly understood [47]. In the light of this, it appears remarkable that simple physical models are even possible. One should realize though that the difficult steps (i.e., sugar loading) have been mostly omitted from these models. Sugar loading still poses a challenge if a more complete understanding of the entire machinery of long range photoassimilate transport is desired.

\subsubsection{The leaf in cross section}

In this section, we focus on the leaf structure, identifying the different internal parts and their spatial relation to each other. This perspective allows us to see the hydraulic transport system that we considered at the whole-tree level in more detail inside the leaf, focusing on the structure of the vascular bundle and its relation to the surrounding tissue. We base the explanations on [2].

Figure 2.8 shows the cross section of a typical dicot leaf. The outer layer is called the epidermis. It covers and protects the more delicate internal structure from the environment (e.g., from bacteria, fungi, or strong sunlight). Embedded in the epidermis are the stomata which allow gas exchange with the surrounding air.

Next, mesophyll tissue consists of the main photosynthesizing cells. In many plants, the mesophyll is separated into the palisade layer, a layer of tightly packed elongated cells directly beneath the epidermis, and the spongy layer beneath it, consisting of less elongated cells with large air spaces between them. The palisade cells' total surface area tends to be much larger than that of the spongy cells.

${ }^{2} \mathrm{~A}$ sort of biological realization of Maxwell's demon. Because energy is spent in the polymerization process, there are no thermodynamical paradoxes. 


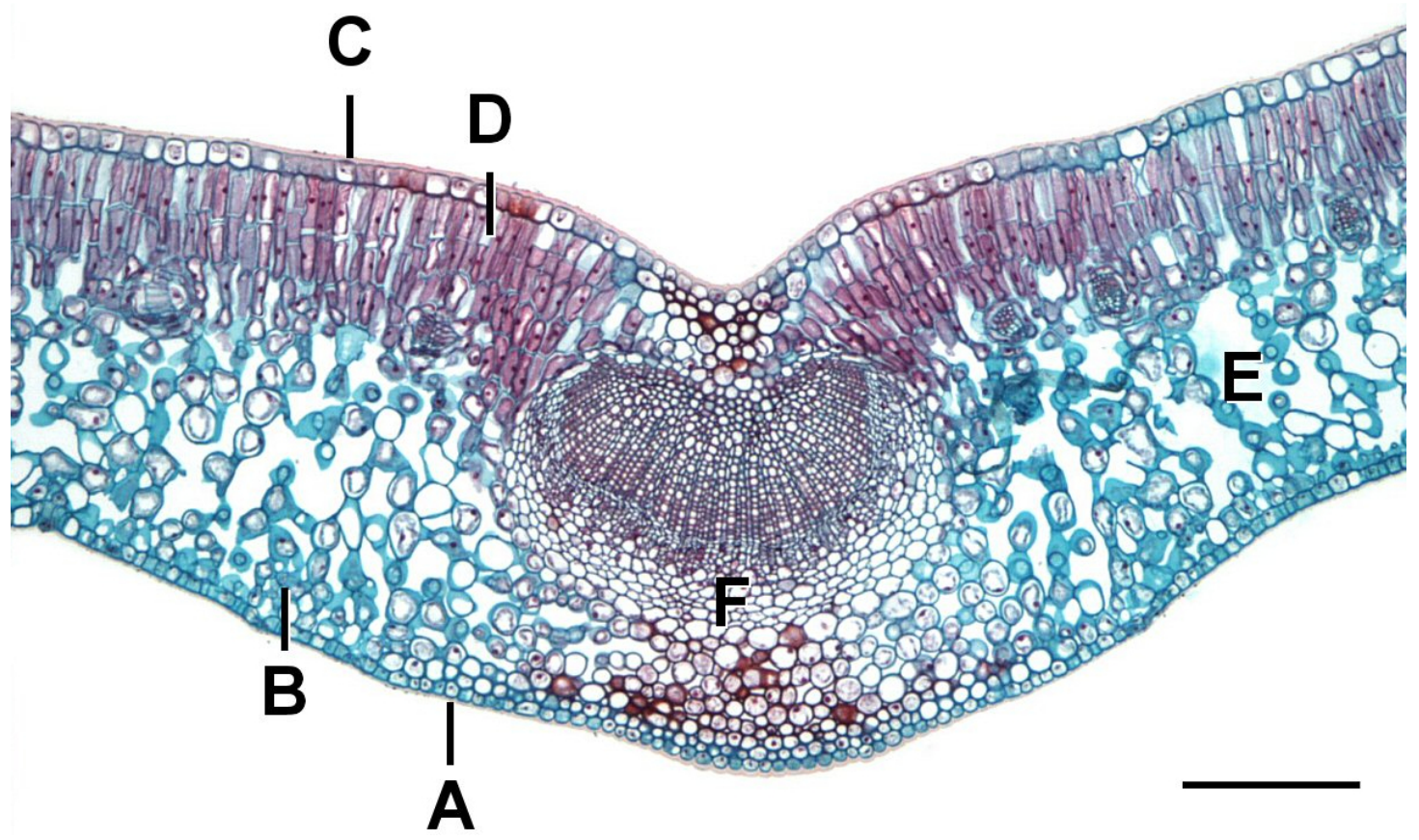

FIGURE 2.8: Cross section of a dicot leaf. The epidermis $(\mathrm{A}, \mathrm{C})$ is the outer protective layer, surrounding the palisade cells (B, D) and the spongy mesophyll (E). Water and photoassimilates are transported in the vascular bundle $(\mathrm{F})$, which also provides structural support. The scale bar corresponds to $0.2 \mathrm{~mm}$. ("Photomicrograph of a dicot leaf" by Jon Houseman and Matthew Ford, CC BY-SA 4.0).

Chloroplasts are also more abundant in the palisade tissue, suggesting that it is the primary site of photosynthesis. On the other hand, the air spaces in the spongy tissue allow for more rapid diffusion of gases.

Finally, vascular bundles are embedded within the mesophyll. Figure 2.9 shows a more detailed cross section of a single vascular bundle. The vascular bundle is divided into several distinct subsystems. We have already discussed xylem and phloem extensively as those tissues which are responsible for transport of water into the leaf and photoassimilates out of the leaf, respectively. They almost always occur together in the same vein (in a number of different patterns, see [48]). The smallest, freely ending veins often contain only xylem [49]. Larger (major) veins are less often integrated in the mesophyll and instead become associated with non-photosynthesizing rib tissue.

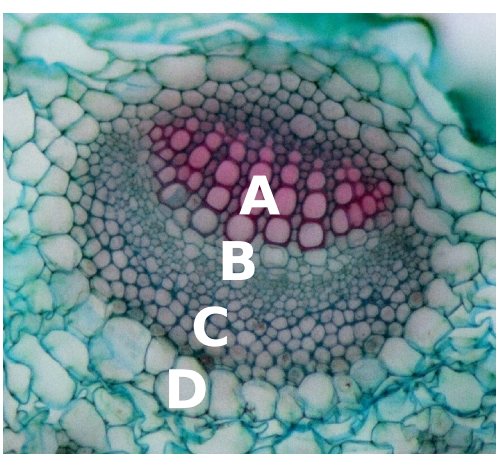

FIGURE 2.9: Cross section of the vascular bundle in a leaf of Taraxacum officinale (common dandelion). Xylem (A) transports water, phloem (B) photoassimilates. Collenchyma (C) gives structural support, bundle sheath cells (D) envelop the vein. ("Taraxacum officinale, central leaf vein, Etzold green" by Micropix, CC BY-SA 3.0 / cropped and annotated). Collenchyma found in the vascular bundle has 
particularly thick cell walls and provides structural stability. Finally, the entire bundle is surrounded by tightly packed bundle sheath cells which control substances entering and leaving the vascular tissue. Still, pathogens such as fungi may penetrate into the vascular bundle and thrive within. The plant is able to compensate by producing additional vascular tissue [50].

Some plants (in particular many grasses, among them many economically important crops such as corn and sugarcane) employ a particularly efficient pathway for photosynthesis (called $C_{4}$ photosynthesis) that is reflected in a markedly different arrangement of the leaf parts and in particular the vascular bundle, leading to more efficient transport of water and photoassimilates (for details see [2]).

\subsubsection{The shape of a leaf}

In this section, we describe the overall outer leaf morphology. In particular, we note the extreme variability in shape of the lamina, as well as the similarly high variability in the organization of the visible venation network.

The leaf typically consists of a short stalk connecting it to the rest of the plant called petiole as well as a broad, flat portion called the leaf blade or lamina (see Figure 2.11). Sometimes, the petiole is missing. Leaves can be simple or compound, i.e., the lamina consists of smaller leaflets that are typically attached to the rachis, an extension of the petiole. Sometimes, the rachis is missing, and sometimes it contains smaller petiolules from which even smaller sets of leaflets arise.
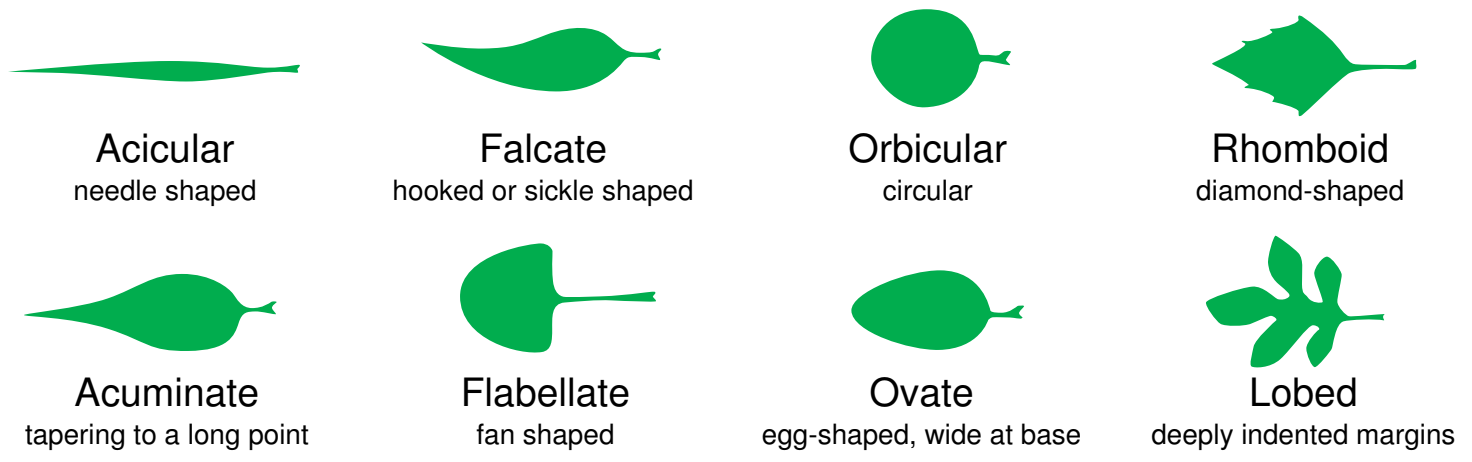

FIGURE 2.10: Examples of various leaf shapes and their nomenclature. Many more possible shapes exist, presenting adaptive trade-offs between many factors. ("Chart of leaf morphology characteristics" by McSush, CC BY-SA 3.0 / cropped and rearranged).

The possible shapes of a single leaflet or simple leaf are manifold, and an extensive amount of nomenclature has been developed to classify leaf shapes [51]. Important characteristics of the lamina are the appearance of lobes and the type of margin (which can be serrated in various ways or not). Figure 2.10 shows some examples of the variety found in nature. Some leaves even fall out of the standard classification completely, such as those of carnivorous plants 
whose leaves are adapted in various ways to catch and digest small prey animals.

Inside the lamina, the venation network is often conspicuous. In angiosperms, it typically consists of one main vein running from the petiole to the tip. Branching off of it are the secondary veins, sometimes ending freely, but sometimes connected to each other through anastomoses. In the space between the secondaries, higher order veins are embedded in a highly reticulate, hierarchically organized fashion. At the highest level, small veins enclose polygonal patches of lamina, the areoles. Sometimes, even smaller veinlets can be found inside the areoles, but these are not reticulate and end freely in the mesophyll tissue. Similar to the overall shapes, a vast nomenclature classifying the venation structure at all levels exists [51]. We will discuss the venation network in more detail in Section 2.1.8.

Functionally, many explanations for leaf shape have been brought forth. They include thermoregulation, hydraulic constraints, biomechanical constraints [52], and even drag resistance [3]. The relative importance of these possible explanations is unclear, conventional wisdom identifying thermoreg-

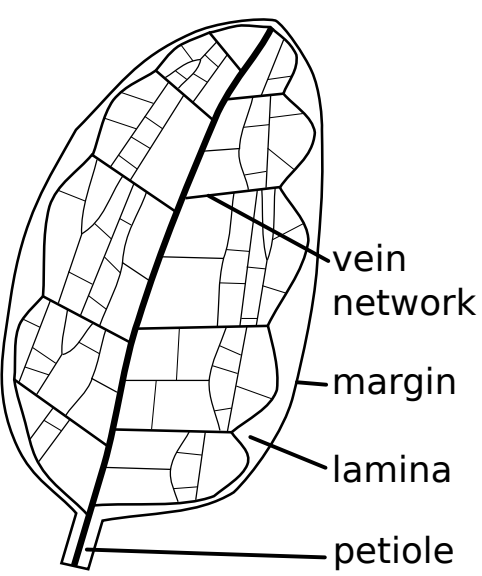

FIGURE 2.11: The basic shape of a leaf. The petiole connects the lamina with the rest of the plant. In this example, the margin is entire and shows no serrations. The hierarchical vein network is embedded in the lamina. ulation as the most likely driver of leaf shape evolution [52]. Because the leaf performs several functions at once, leaf shape evolution is likely driven towards a trade-off solution.

\subsubsection{Structure and hydraulics of the leaf venation network}

In this section, we discuss the structure of the dicot leaf venation network, first in a descriptive manner, then in the context of leaf economy and robust optimal hydraulics. In the context of the whole tree as an integrated system (but ignoring the details of leaf venation), great descriptive success came from scaling and minimum hydraulic resistance arguments. West-Brown-Enquist theory is able to predict allometric scaling laws in animals [53] and plants [54, 55] but fails when it comes to leaf networks, presumably due to the assumption of a treelike, space-filling network.

As discussed above, the leaf venation network is mostly embedded in the leaf mesophyll tissue and consists of the vascular bundles. It is effectively twodimensional, i.e., veins do not cross over each other. Instead, they form junctions where branching occurs. Topologically, these are almost always $T$-junctions, where one parent vein branches into two daughter veins.

In a typical dicot leaf (see Figure 2.12), there is one main vein (or rarely several) which connects the leaf network to the vascular network in the rest of the plant through the petiole. Branching off from it, there are secondary veins which 


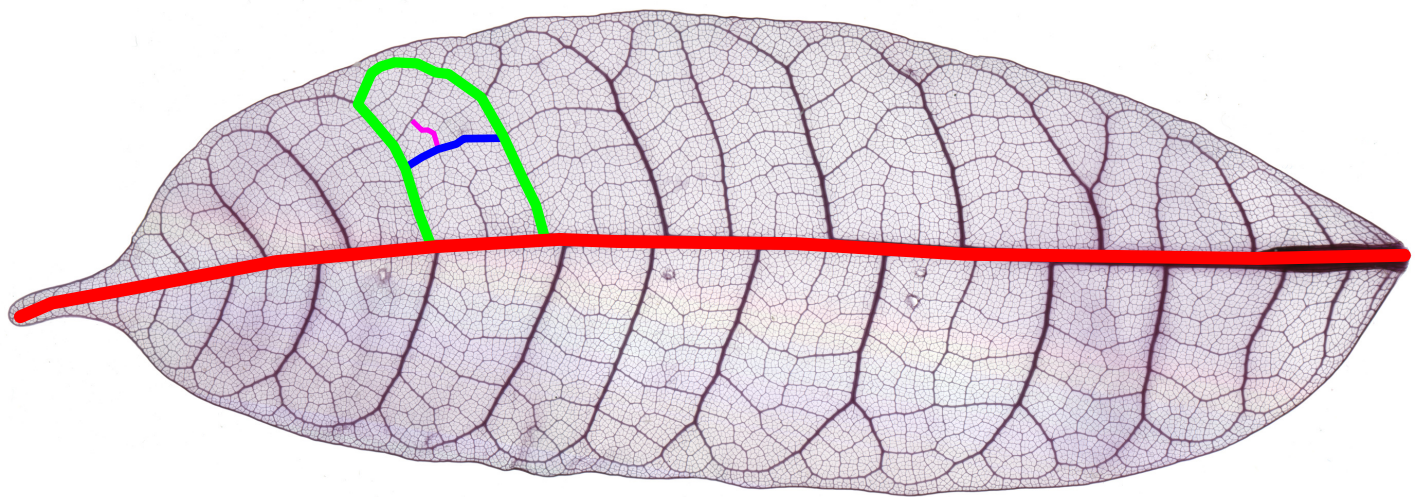

FIGURE 2.12: Hierarchical organization of the venation network in a leaf of Protium wanningianum (the leaf was chemically cleared and the network stained). Branching off from the main vein (red) are the secondaries (green). Third order veins (blue) connect the secondaries, and fourth order veins (purple) the third order veins. The network is reticulate with hierarchically organized loops.

are often connected through anastomoses. From the secondaries, third order veins branch off, and so forth. The different vein orders are characterized by decreasing typical width [51].

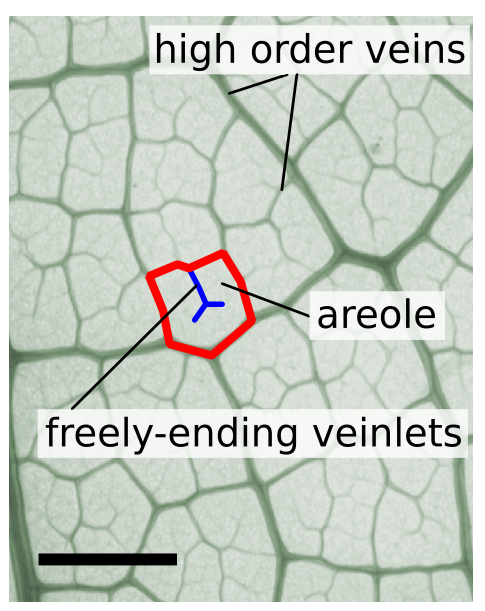

FIGURE 2.13: Highest order venation network in a leaf of Protium grandifolium. Even at the highest level, veins are nested hierarchically. The smallest loops (areoles) enclose treelike veinlets that end freely in the mesophyll. The scale bar corresponds to $1 \mathrm{~mm}$.

The highest order of veins that can be well recognized is associated with the leaf rank, a crude, semi-quantitative measure of network complexity that heavily relies on human learned recognition of form $[56,57]$. Again, there is a vast nomenclature for classification of venation diversity at all orders [51]. Despite this fact, the functional relevance of this structural diversity is unknown.

Instead of focusing on the branching structure of the network, it is possible to consider the loop nesting structure $[58,59]$, recognizing that the network consists of hierarchically nested loops starting from the outer margin which is subdivided by the main vein and descending all the way down to the smallest loops, the areoles (see Figure 2.13). This idea will be the main driver behind the work in Part II, and we shall expand on it there.

Recently, the idea of the leaf economics spectrum was been brought forth, connecting whole-leaf traits such as mass per area and photosynthetic assimilation rates to habitat and climate, and identifying the key investment trade-offs that evolution has to work with in leaves in order for species to remain competitive by maximizing leaf carbon gain $[60,61]$. Even though the leaf economics spectrum is based on summary, whole-leaf traits, the venation network is intimately linked to most 
of them. Thus, a "low-level" description of the leaf economic spectrum can be obtained from geometric venation traits such as areole size, vein density, and vein distance [62]. Developmental models further link geometric traits of the mature leaf to development [63].

This leads to the general idea of a phenotypic space describing leaf venation characteristics. The work described above has done the first steps of mapping out such a space, but the geometric leaf traits analyzed so far tend to be highly correlated due to developmental effects [63]. It is one of the aims of this thesis to extend the known phenotypic space of leaf venation, identifying new, uncorrelated traits in the topological structure of the network.

The topology of the vascular network dictates many aspects of its function. The leaf network can be seen as a collection of hydraulic resistors in the sense of CT theory (see Section 2.1.2.3). The xylem contributes to the total leaf conductivity via [64]

$$
\frac{1}{K_{\text {leaf }}}=\frac{1}{K_{\mathrm{x}}}+\frac{1}{K_{\mathrm{ox}}}
$$

where $K_{\mathrm{x}}$ is total xylem conductivity and $K_{\mathrm{ox}}$ is total outside-xylem conductivity (this includes hydraulic pathways from the vascular bundle through the mesophyll and then through the stomata, see also the simplified model in Figure 2.6). It should be noted that the xylem conductivity empirically appears to be of the same order of magnitude as the outside-xylem conductivity (in [64], a ratio of $K_{\mathrm{x}} / K_{\mathrm{ox}} \approx 6$ was found). Thus, the effect of $K_{\mathrm{ox}}$ on $K_{\text {leaf }}$ is slightly greater than that of $K_{\mathrm{x}}$.

One interesting question is how the venation network should be organized to confer the maximum fitness advantage to the plant. In the context of animal vasculature, Murray's law was brought forth as a guiding principle [65, 66]. It relates the radii of branching vessels according to

$$
r_{p}^{3}=r_{d_{1}}^{3}+r_{d_{2}}^{3}
$$

where $r_{p}$ is the radius of the parent branch and $r_{d_{i}}$ the radii of the daughter branches. ${ }^{3}$ It is derived under the assumption that blood is a costly substance that should be moved under minimum energy expenditure. For the xylem in plants, this assumption does not seem to be valid as water is not costly and the CT mechanism is externally powered by the sun, requiring no energy expenditure [68]. Interestingly, it still appears to hold [69]. As we will show in Chapter 9, the geometry of the phloem can be explained well by minimum energy expenditure arguments (phloem sap is indeed costly and the Münch mechanism does require expenditure of energy). A different model proposes that the network is arranged in such a way as to minimize water stress, meaning the network supplies all parts of the leaf with sufficient amounts of water $[68,70]$.

${ }^{3}$ Leonardo da Vinci already developed a similar rule for tree branching. He proposed the relation $r_{p}^{2}=r_{d_{1}}^{2}+r_{d_{2}}^{2}$ from the principle of conservation of area [67]. 
Equivalently, the network can be understood to minimize the average pressure drop on the leaf blade because a more homogeneous distribution of hydrostatic pressures results in less water stress. In the case of one-dimensional pine needles, this model was successfully used to explain xylem geometry [71]. The total cross-sectional area $A(x)$ of the xylem was shown to scale as

$$
A(x) \sim x^{1 / 2}
$$

where $x$ is the distance from the tip.

Combined with the requirement of robustness against damage, the idea has led to remarkably realistic simulated 2D network topologies [72]. The model minimizes the objective function

$$
\Delta p=\frac{1}{N} \sum_{i=0}^{N}\left(p_{i}-p_{0}\right)
$$

under a fixed-cost constraint for the amount of material invested in the network. Here, $p_{i}$ is the hydrostatic pressure at node $i$ of a planar network containing $N+1$ nodes (node 0 is the petiole). This objective function can be shown in the limit of uniform evaporation on the leaf blade to be equivalent to both

$$
\begin{aligned}
& \Delta p \sim P=\sum_{e} \frac{F_{e}^{2}}{K_{e}} \\
& \Delta p \sim \frac{1}{K_{\mathrm{x}}},
\end{aligned}
$$

where the sum in (2.13) runs over all edges $e$ in the network and $F_{e}$ is volume flow through edge $e$. Thus, (2.13) is the total viscous power dissipation in the network. The simple objective function (2.12) can be modified to take into account robustness against damage. An example simulated network is shown in Figure 2.14. We will extend these ideas slightly in

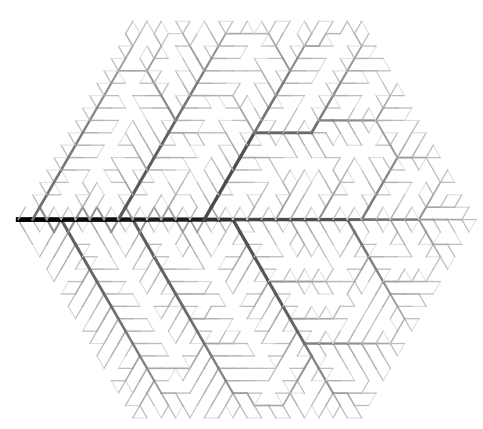

FIGURE 2.14: Example simulated leaf network using the damageresistant model from [72]. Many of the features of real leaf networks (compare to Figure 2.12) are present such as hierarchy of main, secondary and higher order veins, anastomoses connecting secondaries, and hierarchically nested loops. Chapter 10.

Thus, the venation network optimizes three objectives at the same time: Pressure drop, hydraulic conductivity, and power dissipation. Pressure drop and hydraulic conductivity are connected with efficient xylem operation and power dissipation is linked to the phloem (see Chapter 9).

The fact that both vascular systems almost always occur in the same vascular bundle (see also Figure 2.9) may be a reflection of this multiple optimality, and an example of the principle of parsimony in nature: There is no need to build two different networks if the same network architecture optimizes transport 
through both xylem and phloem.

\subsection{9 "Design" in nature and the constructal law}

Many natural systems (by which we mean systems on all scales, e.g., forests, trees, leaves, venation networks, etc...) have the appearance of design. Of course this is a wrong observation because evolution through natural selection is non-teleological [73]. Still, as a metaphor it is useful to speak of functional "goals" and "design" of natural systems. The principles of optimization (of optimal design) described above can be put into the larger context of the constructal law, which stipulates that there exists a global architecture facilitating cyclical flow of water (in the plant-atmosphere-river-soil continuum) as well as flow of mechanical stresses (from atmosphere to ground) [74, 75]. The constructal law can be seen as a guiding principle for the appearance of design in nature. Any natural system should be expected to optimize flow. This leads to the prediction of some well-known allometric scaling laws [76] such as Kleiber's law, stating that an animal's metabolic rate scales as $M^{3 / 4}$, where $M$ is its mass [77]. It also puts the objective functions we used to explain venation structure into a broader context.

It should be noted that the constructal law is not a fully fleshed-out mathematical theory of biological systems but rather a set of (vague) ideas that may guide the modeler in the right direction.

\subsection{Developmental leaf biology}

In this section, we discuss the developmental biology of the dicot leaf. First, we describe the emergence of leaves from specialized tissue found at the tip of the shoots, the shoot apical meristem (SAM). The SAM contains totipotent stem cells able to differentiate into any plant tissue and produces leaf "embryos," the leaf primordia. Next, we describe the growth of the primordia in several phases during which the vascular system is differentiated. The growth of vascular tissue (and many other plant tissues) is thought to be regulated by the phytohormone auxin. Finally, for completeness, we discuss the slightly different growth mechanism of gymnosperm needles.

\subsubsection{Leaf initiation and the shoot apical meristem}

The shoot apical meristem is a collection of undifferentiated (meristematic) cells found at the very tip (apex) of a shoot. Its function is to act as the initial site of growth and formation of new plant organs such as leaves and flowers. At the very center of the SAM (the central zone), slowly dividing stem cells continuously replenish the supply of tissue which migrates away from the apex to the peripheral zones. There, organ initiation takes place. Even though there is a 
constant flux of cells as well as budding off of organs, SAM size and organization remain unchanged [78].

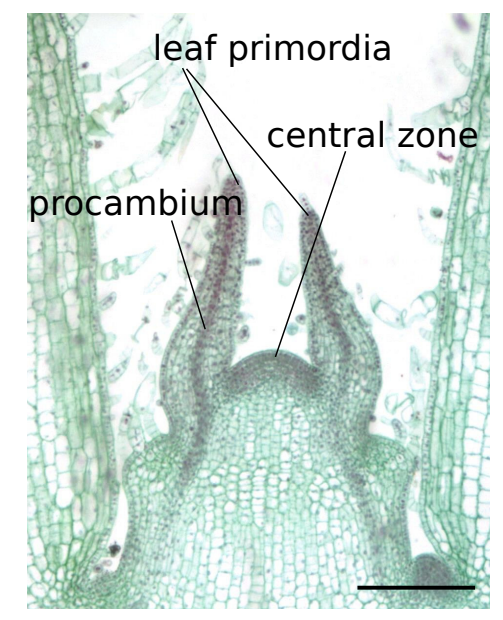

FIGURE 2.15: Shoot apical meristem of Coleus sp. Two leaf primordia have been formed by the meristem tissue. Inside the primordia, vascular tissue (procambium) has already differentiated. The scale bar corresponds to $0.2 \mathrm{~mm}$. ("Photomicrograph of a Coleus stem tip" by Jon Houseman and Matthew Ford, CC BY-SA 4.0 / cropped and annotated).

suppression that later develop into the distinct serrations and folds. tion [79-83]. both xylem and phloem are derived.

Figure 2.15 shows a micrograph of a SAM where the central zone as well as two leaf primordia can be discerned. As they grow, leaf primordia move downward (from the perspective of the SAM), forming intricate phyllotactic patterns. A combination of mechanical stresses and hormonal regulation is believed to be responsible for both initiation of the primordia and their phyllotactic spatial organiza-

The formation of a new primordium is accompanied by an accumulation of the plant hormone auxin which is transported actively towards the site of initiation. Once initiation is complete, transport reverses away from the primordium which now acts as a source of auxin [84]. Inside the growing primordium, auxin is believed to be the main driver of vascular development [84, 85], responsible for differentiation of cells into procambium, from which

In leaves with an entire margin, two distinct narrow bands (the marginal meristem) form, which develop into the leaf blade. The tissue between them develops into the main vein [2]. In lobed or serrated leaves, the marginal meristem is further subdivided into alternating areas of growth enhancement and

\subsubsection{Vein morphogenesis}

The development of the vascular system in angiosperm leaves proceeds in several overlapping phases $[63,86]$. In the first phase, growth in the leaf primordium occurs primarily by cell division. In this phase, the main vein and the secondary veins are formed, starting from the tip of the leaf downward to the petiole. In the second phase, growth is mainly by cell expansion. Now, minor veins successively subdivide the space between higher order veins, forming an intricate network (see Figure 2.17).

Similar to the process of leaf initiation at the SAM, vascular differentiation is believed to be controlled mainly by flow of the hormone auxin, even though the precise mechanism is unknown $[84,85,87,88]$.
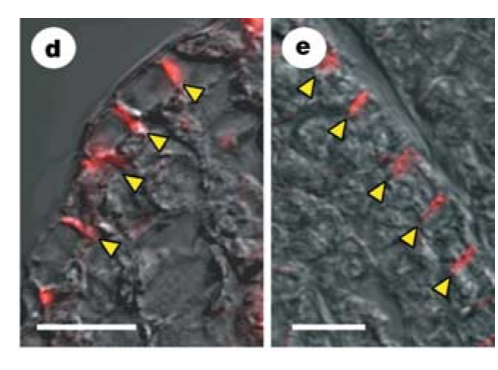

FIGURE 2.16: Polar accumulation of PIN in a leaf primordium of Arabidopsis. PIN is marked in red. Scale bars correspond to $10 \mu \mathrm{m}$. (From [82], reprinted with permission). 

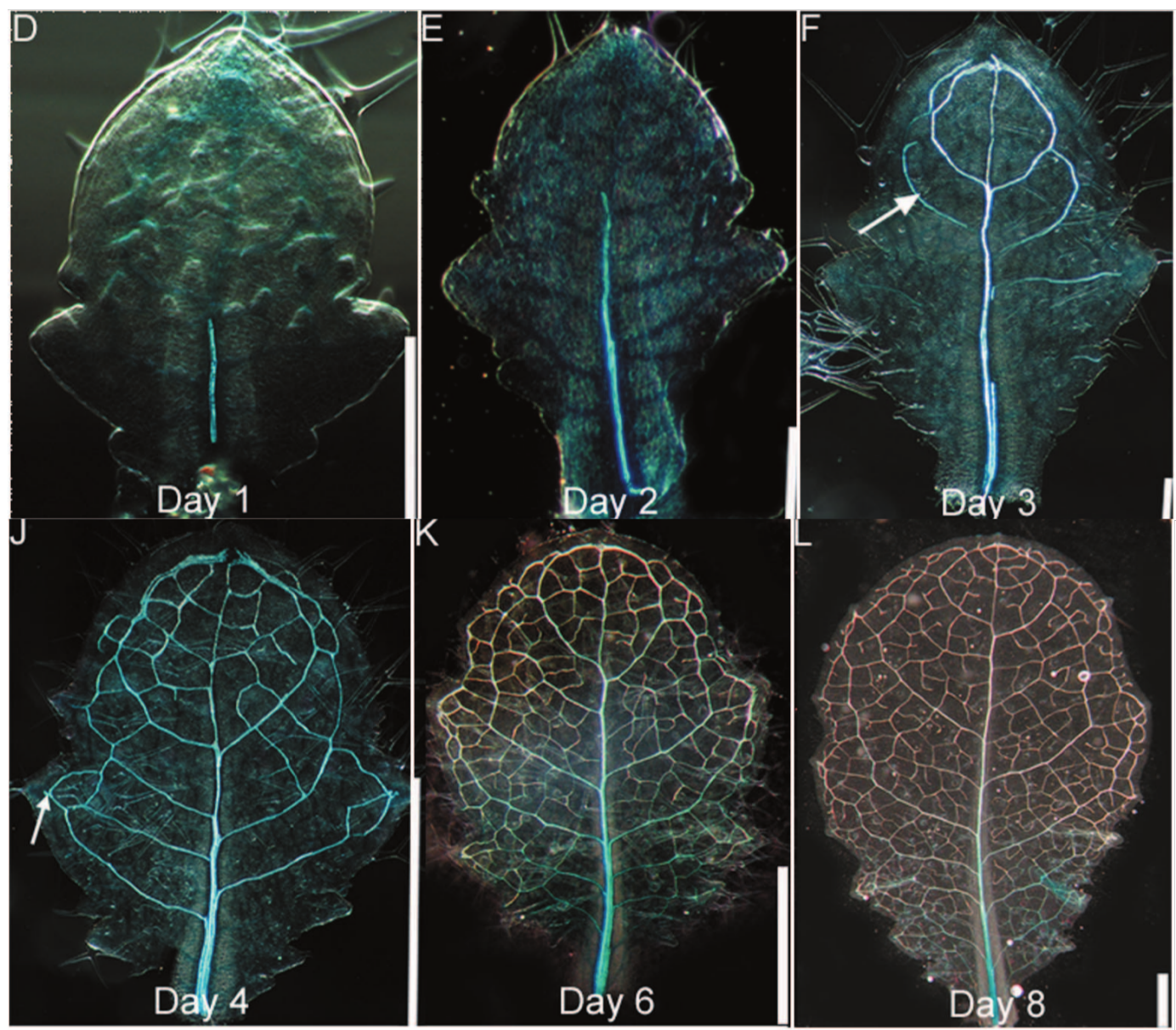

FIGURE 2.17: Development of the vascular pattern in Arabidopsis thaliana. D-F, J-L, dark field microscopy of differentiated xylem. Development of a cotyledon is shown from day 1 to day 8 after germination. The main vein differentiates from bottom to top, higher order veins differentiate from top to bottom. D-F, scale bars correspond to $200 \mu \mathrm{m}$, J-L, scale bars correspond to $1 \mathrm{~mm}$. (From [86], reprinted with permission, (C) 2004 by The University of Chicago. All rights reserved.)

It is generally believed that auxin is produced in special cells within the developing lamina from which it moves away by diffusion. The petiole acts as a sink. In addition to diffusion, it has been shown that auxin is actively transported through plant cells by means of a class of efflux carrier proteins called PIN. They accumulate polarly in the cell walls (see Figure 2.16), facilitating transport of auxin from one cell to the next [89-91]. Cells sense either auxin concentration or auxin flux which triggers differentiation into vascular tissue. Increased flux leads to more differentiation, which in turn further increases flux. This positivefeedback mechanism is called auxin canalization theory and is, together with polar auxin transport by PIN, the main accepted explanation for vascular tissue development. Auxin canalization theory is not without criticism, and models have been brought forth that invoke mechanical effects as drivers of vein pat- 
terning [92-95]. One fundamental problem in assessing such models is the high difficulty of directly measuring auxin in the developing leaf.

Mathematical models of vascular development are plentiful, the first being Mitchison's seminal contribution [96]. The review [85] alone lists 16 different approaches, many with only minor differences. Fundamentally, the models can be divided into two classes: flux-based and concentration-based, differing in which quantity (flux or concentration of auxin) the cell measures and adapts to. The large number of models shows a fundamental difficulty, it is very hard to verify (or falsify) any of them because their predictions are largely similar, and no sufficiently sensitive experimental techniques exist to rule out at least some of them.

A simple way to model the flux of auxin through a cell wall connecting two cells is [97]

$$
F=D \Delta c
$$

where $\Delta c$ is the difference in auxin concentration and $D$ is an effective diffusion coefficient (e.g., incorporating facilitated transport via PIN but no effects of polar transport) that depends on flux through

$$
\frac{d D}{d t}=\alpha F^{2}+\beta-\gamma D,
$$

where $\alpha, \beta$, and $\gamma$ are parameters. Equation (2.16) describes a positive feedback mechanism. If the flux is large, $D$ increases, further facilitating higher flux. Similarly, if the flux is small, $D$ decays, shutting off the connection. Most fluxbased models are variants of this basic idea, either using a different feedback function (some $f(F)$ instead of $\alpha F^{2}$ ) or more sophisticated models for facilitated diffusion. Concentration based models tend to be more complicated as they often incorporate various additional processes such as auxin influx, efflux, saturation, production and degradation. A comparatively simple one-dimensional model is described in [98]. It is interesting to note that equation (2.15) is formally equivalent to cohesion-tension theory upon replacing the effective diffusion constant by conductivity and concentration by hydrostatic pressure. We discuss the general theory of such linear flow models in Chapter D of the Appendix.

One major modeling question is how to explain the formation of closed loops in the venation system. The "bare bones" models result in networks that are topological trees, and one needs to add additional "bells and whistles" to make closed loops persist $[99,100]$. In Part III, we show that fluctuating sources and sinks can account for closed loops. Despite continuous work over the past decades, the morphogenesis of vascular networks in leaves is so far not wellunderstood. 


\subsubsection{Global geometry and folded growth}

The global geometry of plant leaves, i.e., the overall shape, is extremely variable (see Figure 2.11). As discussed above, it has been explained as an adaptation to minimize air drag or maximize convective cooling of the lamina. However, it is important to take into account possible developmental constraints. Two basic developmental modes exist. The so-called pre-formed leaves grow and develop within a protective bud. Many temperate species of trees employ this strategy, presumably to be able to have functional leaves immediately available upon start of the growing season and to take advantage of good conditions in the previous season [101]. In contrast, neo-formed leaves do not grow inside a bud and can expand freely. Many tropical and subtropical species employ this strategy.

It has been recently observed that there is a rich interplay between pre-formed folded growth and leaf shape [102-104]. In essence, it is possible to predict the lobed leaf shape as well as serrated margins of many temperate tree species (e.g., hornbeams, or maple) by the constraint that the folded leaf needs to be space-filling inside the bud. Additionally, leaf shape is closely linked to vascular patterning [105]. Lobes and serrations in the margin act as points of termination for veins and, during development, as localized auxin sources [90]. This is also supported by simple computer simulations [106].

Two basic venation patterns have often been

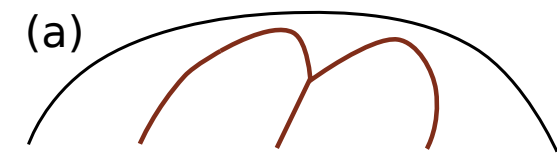

(b)

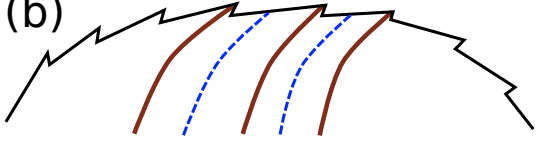

FIGURE 2.18: Venation types and folded growth. Secondary veins are in brown. (a) Brochidodromous venation, related to neo-formed leaves. (b) Craspedodromous venation, related to folded growth. Blue dashed lines are folds. used as proxy for climate. In brochidodromous leaves, secondary veins are connected by thick anastomoses. Such leaves are often found in tropical climates. In craspedodromous leaves, the secondary veins terminate in the serrations of the leaf margin and are not connected by anastomoses. Such leaves are predominantly found in temperate regions. Folded growth provides an explanation for this, as the temperate species' pre-formed leaves could be subject to developmental constraints preventing anastomoses and promoting lobes and serrated margins (see Figure 2.18). Limited studies in species that produce both pre-formed and neo-formed leaves appear to support this [107, 108].

\subsubsection{The gymnosperm needle}

In gymnosperms and monocots, leaf formation proceeds differently from the angiosperms. If one compares the process in angiosperms with drawing on an expanding balloon, where the network is formed in the embryo and then later continually added upon as the leaf lamina expands, gymnosperms can be more aptly compared to an inkjet printer. After leaf initiation, the primordium becomes hood-like, enclosing the central zone of the SAM. The zone of growth is restricted to a small band where the lamina attaches to the SAM. New cells are added in a 
linear fashion to the growing leaf from the bottom [2]. Because of this process, the venation network is not nearly as intricate as in dicots. Gymnosperms tend to form only a single or double midvein, monocots form a set of parallel veins running from the tip of the leaf to the petiole, interconnected by smaller veins running perpendicularly.

Thus, the topology of the monocot and gymnosperm venation network is much less rich than that of the angiosperms. Still, this simplicity allows for a mathematical treatment of the flow processes that can admit analytical solutions. In the case of the phloem in conifer needles (gymnosperms), we show in Chapter 9 that a very simple optimization model can explain the observed pattern of total cell area.

\subsection{Evolution of leaf venation}

In this section, we describe the evolution of the angiosperm leaf venation network. The unique structure of their venation network has been a major contributor to the angiosperms' evolutionary success. Today, they are the dominant land plants on earth, having replaced the conifers around 60-100 million years ago. This section is based on the reviews [49, 68, 109], a survey of extant and extinct venation patterns in plants is [110].

\subsubsection{Evolution of land plants and leaves}

From the first appearance of land plants in the Ordovician (ca. 450 million years ago), atmospheric $\mathrm{CO}_{2}$ fluctuated strongly. The lowest concentration was found during the late Carboniferous (ca. 300 million years ago) and the highest when the first land plants appeared. High $\mathrm{CO}_{2}$ conditions favored relatively low hydraulic conductance and high water storage capacity, both features found in the earliest plants. However, even at optimum conditions, there still was a need for water transport. The CT mechanism is likely to have evolved rather directly, as the driving force comes for free and all it takes is a hydrophilic cell wall to act as a wick for water. The precursors for such a mechanism would have been present in the primitive algae that terrestrial plants evolved from [109]. Thus, the basic transport tissue had already evolved by the time the leaf arose.

The earliest land plants lacked leaves. Yet, by the mid Carboniferous (ca. 320 million years ago), leaves had already independently evolved five or more times. Macrophylls (i.e., leaves with multiple veins) likely arose as lateral branches that exhibited in-filling, or webbing, between branches in the same plane [49]. Because of the origin of venation from branches, all early venation was topologically treelike, without loops. The same venation topology can be seen today in the "living fossil" Ginkgo biloba. Simple reticulate venation and hierarchical organization evolved in ferns and early gymnosperms (e.g., in Gnetum) during the Upper Carboniferous, but did not reach the sophistication of the later angiosperm system. 


\subsubsection{Evolution of the angiosperm venation network}

During the early and mid Cretaceous (between 145 and 66 million years ago), the angiosperms rapidly began to dominate all land [111]. This "angiosperm radiation" was so extreme that even Charles Darwin found it at odds with his theory of gradual evolution, even calling it an "abominable mystery" [112]. ${ }^{4}$ The rapid expansion of the angiosperms is closely linked with falling atmospheric $\mathrm{CO}_{2}$ concentration during and directly before this extreme event. This is thought to have put the early angiosperms under considerable selective pressure to improve their $\mathrm{CO}_{2}$ exchange capabilities. This was primarily achieved by increasing stomatal conductance $g_{s}$. In order to minimize the bottleneck length scale of diffusive transport, the stomata became smaller but increased in number [111].

In order to efficiently supply a large number of stomata with water, vein density (total vein length per area) increased in two phases during the Cre-
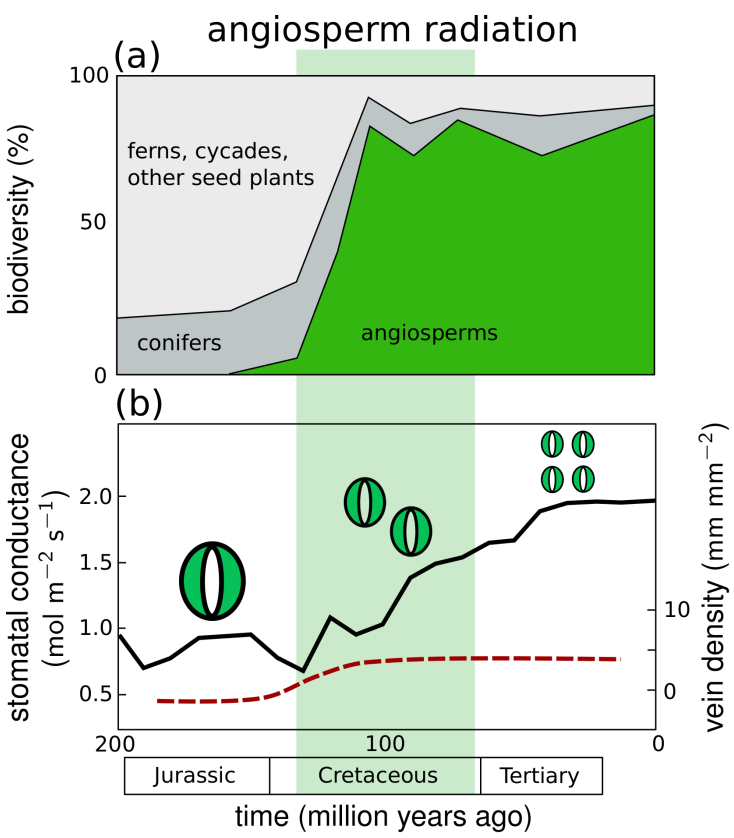

FIGURE 2.19: The angiosperm radiation event in evolutionary time (green shaded region). (a) Relative abundance of plant groups (filled regions). (b) Angiosperm stomatal conductance (black line) and reconstructed vein density (red dashed line). (Redrawn and simplified after [111]).

taceous, first during the Late Albian (ca. 100 million years ago), when the nonangiosperm maximum density $\left(6 \mathrm{~mm} \mathrm{~mm}^{-2}\right)$ was surpassed, and then again at the border between Cretaceous and Tertiary (ca. 60 million years ago), when the first modern values of the vein density were observed [111] (on the order of $10 \mathrm{~mm} \mathrm{~mm}^{-2}$ ). This massive increase was achieved through the highly reticulate, hierarchically organized venation topology we find today. The timeline of the angiosperm radiation event together with the environmental conditions is shown in Figure 2.19.

\subsection{Summary}

In this introduction, we discussed aspects of function, development, and evolution of the dicotyledonous plant leaf with special focus on physical modeling. We began by summarizing the numerous functions a leaf needs to accomplish effectively in order for the plant to survive and thrive. We then described the

\footnotetext{
${ }^{4}$ Now that we have a much better fossil record, the apparent incompatibility with the theory of evolution has all but vanished [113].
} 
basic theory of photosynthesis and how it relates to water and nutrient transport within plant and leaf. Further, we gave a description of the cohesion-tension theory of passive, evaporation-driven water movement through the plant. We discussed the xylem as water transport tissue as well as the mathematics behind CT theory in terms of local (vein level) and global (whole tree level) models of hydraulic resistors. Next, we discussed the Münch mechanism of active, osmoticgradient driven photoassimilate transport through the phloem. Following, we gave a short overview over the structural biology of a leaf in cross-section, bringing the vascular tissue into context. We then discussed aspects of leaf shape and presented a global view of the leaf venation network and its hydraulics. We especially focused on the fact that the venation network appears to be highly optimized by evolution for its task of water and photoassimilate transport. Following, we discussed development of the dicot and monocot leaf from the shoot apical meristem as well as vein morphogenesis and simple auxin canalization models. Finally, we turned to evolution, giving a brief history of the dicots and their venation network, focusing specifically on the evolutionary advantage and eventual success of the modern efficient and highly dense, reticulate venation network. 



\section{Part II}

\section{Topological Phenotypes in Leaf Vascular Networks}





\section{Phenotyping Leaf Venation Networks}

As we saw in the introduction, transport networks are of critical importance to the efficient and robust functioning of biological systems. In man-made systems, optimality is achieved through careful design and engineering. In biological systems such as leaf networks, evolution through natural selection appears to drive vein patterning towards networks that tend to optimize certain proxies for biological fitness (e.g., energy expenditure), see $[72,114]$ as well as Part III of this thesis. In the case of leaf venation, the optimized network has a highly reticulate, hierarchically nested topology that certain models are able to reproduce (see Part III). However, comparisons between model topology and real leaf networks
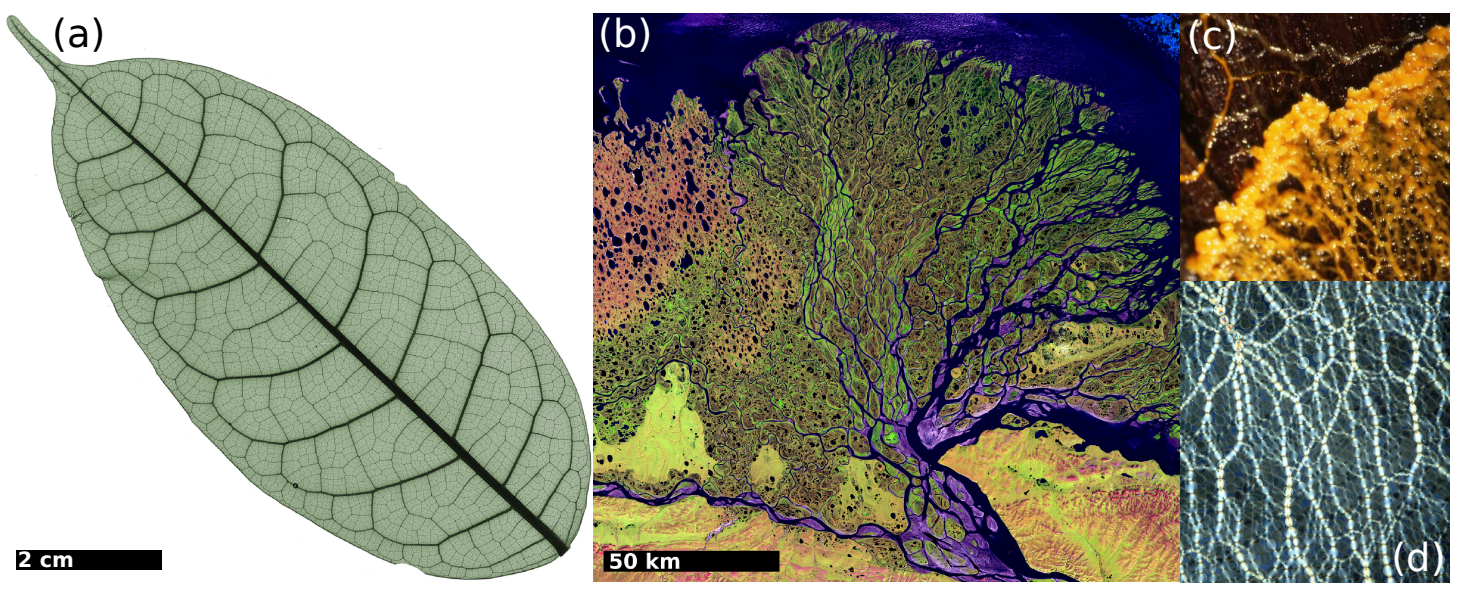

FIGURE 3.1: Examples of reticulate topology in natural and man-made networks. (a) Cleared leaflet of Protium nervosum from the database analyzed in this thesis. The highly reticulate, hierarchically organized venation topology is clearly visible. (b) False color composite of the delta of the river Lena, flowing into the Arctic Ocean. Thermal erosion due to the local seasonal variability (for seven months of the year, the delta is frozen tundra, the rest of the time, wetland) has resulted in the particular, highly reticulate topology of the river delta network [115]. (c) The slime mold Physarum psittacinum forages for food by producing a network of cytoplasm-filled tubes. (d) Jammed photoelastic disks show reticulate force-chains. (b) by NASA Landsat 2000, in the public domain. (c) "Physarum psittacinum" by Helen Ginger, CC BY-SA 3.0 / cropped. (d) From [116], reprinted with permission.)

still largely rely on the capability of humans to detect similar patterns. In this part of the thesis, we make a contribution towards producing a quantitative tool which is able to encode the topological structure of planar, reticulate, weighted networks such as leaves, blood vessels in the brain, river deltas, slime molds, force chain networks in granular materials or even human-made street networks (see Figure 3.1 for examples). The topological metric we use is based on ideas from computational topology [117] for finding persistent cycles in discretized 


\section{Phenotyping Leaf Venation Networks}

data (in particular representations of proteins) which were generalized to the case of hierarchically nested, weighted networks [58, 59, 118].

Leaf venation networks provide an excellent test case for such topological metrics because their diversity and variability is staggering-even within a single family (see Figure 3.2).
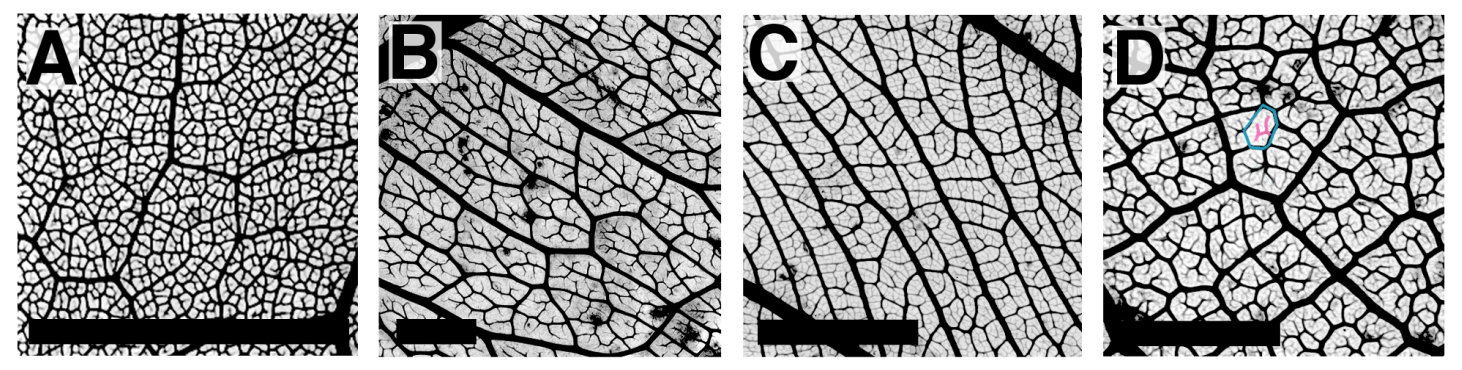

FIGURE 3.2: Examples of the diversity of leaf network morphology in the Burseraceae family. We show the highest order venation morphology in leaf scans from A Protium ovatum, B Protium madagascariense, C Pouteria filipes, D Canarium betamponae. In D, one areole (smallest loop) is marked together with freely ending veins that are typically found inside. Scale bars correspond to $2 \mathrm{~mm}$.

Researchers have developed a variety of geometric metrics characterizing venation properties in adult $[62,63,94]$ and developing leaves [121] and showed that they correlate with photosynthetic efficiency [122-124] (indeed, the ability of dicots to form highly dense, nested venation is thought to be a major contributing factor to their evolutionary success as discussed in the introduction), as well as climate and vegetation type [61, 63, 125, 126]. These metrics typically involve the measurement of quantities such as vein density (total vein length per area), thickness, or areole area. They were shown to scale with leaf size according to a developmental model [63]. Essentially, during morphogenesis, large veins form first during a phase of growth by cell division, whereas smaller veins form later during a partly overlapping phase of growth by cell expansion (see Figure 3.3). Thus, the ge-

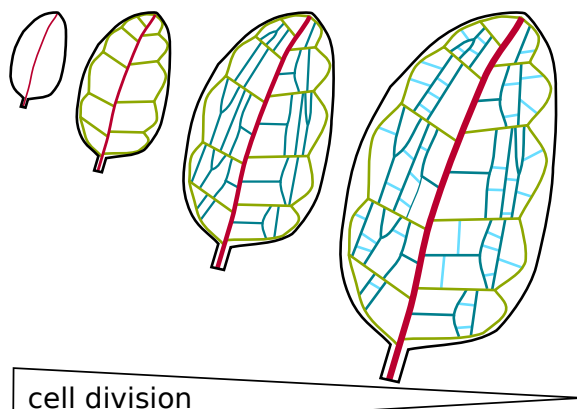

cell division

cell expansion

FIGURE 3.3: Leaf venation morphogenesis in Arabidopsis thaliana [86, 119-121]. Leaf blade morphogenesis is characterized by overlapping phases of (slow) growth by cell division and (fast) growth by cell expansion. Vein orders develop in successive fashion, the main vein being formed first, then the secondaries, then finally higher order veins. (Redrawn after [63]). ometric properties of large veins (such as the main vein) turn out to correlate with leave size whereas the properties of small, higher order veins do not.

Little work exists analyzing the architecture of leaf venation beyond simple geometry, researchers so far having concentrated on a qualitative, descriptive nomenclature [51]. A semi-quantitative approach different from ours was pursued in [56]. However, both these approaches still rely on human inspection 
of photographs or leaf specimens for identification of traits. In this part of the thesis, we develop an automatized quantitative method that is able to extract topological venation traits from cleared, scanned and vectorized leaf networks. The groundwork for this method was laid in [58]. The method focuses on the hierarchically nested nature of the loops that make up the venation and that is characteristic for many transport networks, including leaf venation, and that will be of importance in Part III as well. Our work is the first ever study of these topological leaf traits in a large data set.

In conjunction with geometric traits such as those investigated in $[62,63,121]$, we are able to compute a leaf fingerprint, or quantitative phenotype, from local geometry and topology (i.e., using only information available in the higher order venation pattern) that can be used to improve identification of leaf specimen (or even species) as compared to using geometry alone. With the help of a custom dataset of 186 fully vectorized leaf networks, we use eight local phenotypic traits (or features) comprising the fingerprint, three from topology and five from geometry, and show that the feature space is spanned by two orthogonal dimensions approximately given by geometry and topology (in the sense of Principal Component Analysis and Factor Analysis). Identification of leaf specimen and leaf species from fragments is significantly improved when using topological information, thus establishing the relevance of both the fingerprinting method and our topological metrics. Even though we produce a proof-of-principle instead of a fine-tuned identification system, the basic idea is relevant to both the field botanist and the palaeobotanist, who may only have access to small leaf fragments or leaf litter. Albeit rare, sufficiently well-preserved fossil fragments exist (e.g., [127]). We finally present a simple, empirical developmental model that reproduces the measured topological statistics for a wide range of leaf networks and that is controlled by a set of parameters regulating noise.

The rest of Part II is organized as follows. Chapter 4 introduces the metrics we use to quantify local geometry and topology, most prominently the hierarchical decomposition method used to extract the hierarchical nesting structure of loops [58]. In Chapter 5 we summarize the results of our quantitative analysis of 186 leaves in our data set. Chapter 6 presents a phenomenological growth model that is able to reproduce a wide range of topological statistics measured in the dataset. In addition, Chapter A of the appendix contains an overview over the statistical and machine learning techniques that we used to analyze our data.

The results from Part II were published in part in [128]. 



\section{Metrics for Planar Networks}

In this chapter we discuss metrics that can be applied to the problem of phenotyping planar networks such as leaf venation. We use the word "metrics" to refer to quantitative methods which assign some number to the whole or a part of a network that is chosen to provide a summary statistic of some abstract property.

As discussed before, in the study of leaf networks and phenotyping in general, geometrical metrics have had a long tradition. They can be shown to be linked to photosynthetic efficiency [122-124], high venation density being one of the main factors contributing to the evolutionary success of the angiosperms [109]. Indirectly, geometric metrics are also known to be correlated with climate and vegetation type $[61,63,125,126]$, in particular as part of the global leaf economics spectrum [60]. In addition, they are known to scale with leaf area due to universal properties of morphogenesis (see [63] and Figure 3.3). Here, we concentrate on local traits which can be estimated from a small fragment of the leaf without knowledge of global properties such as total leaf area. In the study of fossilized leaves, often only such fragments exist.

In contrast to geometry, the topological metrics we employ have not been used before in botany. Some unrelated topological metrics have been applied to semi-automatic quantification of leaf venation [56], but these still rely on visual inspection of the network by a human. A qualitative classification of leaf traits exists as well [51] but cannot be easily automated. Our metrics [58, 59] rely on encoding the hierarchical nesting structure of loops in the network as a tree graph and can be computed automatically. This tree graph is then analyzed statistically using methods inspired by neuroscience [129-131], where they were devised to quantify the branching structure of dendrites. As an additional metric, we quantify the topological length of tapered veins.

We use the statistics of our topological metrics to define a statistical distance between local topologies, quantifying the degree of similarity between hierarchically nested structures.

A convenient representation of leaf networks is a weighted planar graph. Such a representation is able to capture the essential features: small stretches of venation between junctions correspond to edges, junctions correspond to nodes, and areoles correspond to facets of the planar polygon associated to the graph. Basic geometry of the network such as vein widths are encoded as weights.

The rest of this chapter is organized as follows. We first describe the graph representation of leaf network geometry which we use. Then, we describe five geometrical leaf traits, following [63] and [62]. The traits are vein density, vein distance, weighted vein thickness, areole area, and areole density. Furthermore, we describe two different topological traits. First, the (weighted) nesting number 
which is derived from the nesting tree $[58,59]$, as well as a detailed description of the hierarchical decomposition algorithm used to construct it. Second, the topological length of tapered veins.

\subsection{Graph representation of leaf venation}

Representing a leaf venation network as a weighted planar graph is a natural choice in many respects. The network is twodimensional and veins do not cross without forming junctions (see also Figure 4.1). For a given leaf network, we can therefore define a graph $G=(N, E)$ consisting of node set $N$ and edge set $E \subset N \times N$. A planar embedding is given by a set of coordinates $\left(x_{n}, y_{n}\right)$ associated to each node $n \in N$. Weights representing vein diameter $a_{e}$ and length $\ell_{e}$ are associated to each edge $e \in E$. The lengths are not necessarily the Euclidean distances between nodes but are the measured actual lengths of possibly curved pieces of venation.

Because of the planar embedding, G contains additional structure as a planar polygon when removing all treelike components (see Figure 4.1). In practice, this is achieved by removing all edges from the graph that cannot be expressed as a linear combination of its topological oriented cycles (for the mathematics of topological cycles see Chapter D of the appendix).

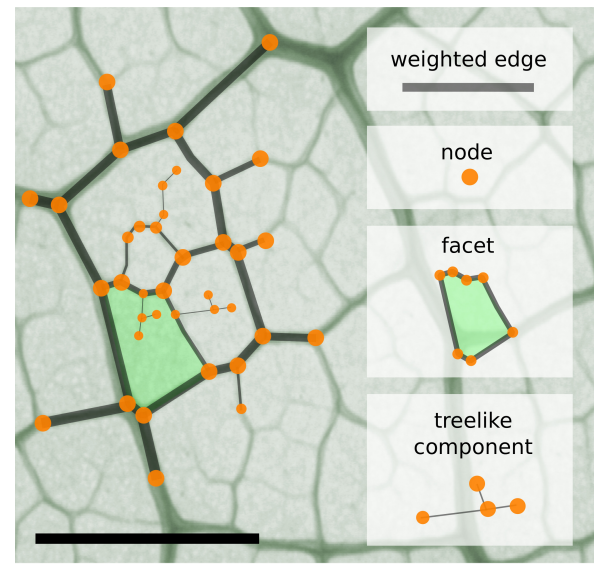

FIGURE 4.1: Partial representation of leaf venation as a weighted planar graph. Venation between junctions is represented by edges (dark lines, line thickness corresponds to vein width), junctions are represented by nodes (orange dots). True venation length (including the effect of vein curvature) is encoded as a second edge weight. The graph can be seen as a planar polygon by removing all treelike components, keeping only the facets (green). The scale bar is $1 \mathrm{~mm}$.

\subsection{Geometric leaf traits}

In this section, we describe all the geometric leaf traits that are analyzed in this thesis. Where appropriate, we also briefly present the algorithms used to calculate them. The most important geometric leaf traits are schematically represented in Figure 4.2. They are the areole area, vein distance, vein density and weighted vein thickness. These metrics have been used extensively, e.g., in [62, 63]. 


\subsubsection{Vein density}

Vein density $\sigma$ is calculated by summing the total length of all veins and dividing by total leaf area or the area of the leaf segment that is being analyzed,

$$
\sigma=\frac{\text { total length of all veins }}{\text { total area }}
$$

\subsubsection{Vein distance}

The mean distance between veins $d$ is not easy to calculate directly from the graph description. Given a binary image of a leaf and the associated Euclidean distance map, one can find the maxima of the distance map inside the areoles and take these as estimates of the vein distance [62]. For computational ease, we use a slightly different procedure that only uses the graph description, but is potentially less accurate.

For each facet $i$ in the network, we first compute the convex hull, and from this the largest inscribed circle (this is now a linear programming problem [132]). This circle's diameter $d_{i}$ is taken as an estimate of the vein distance. The mean vein distance is now simply the mean over

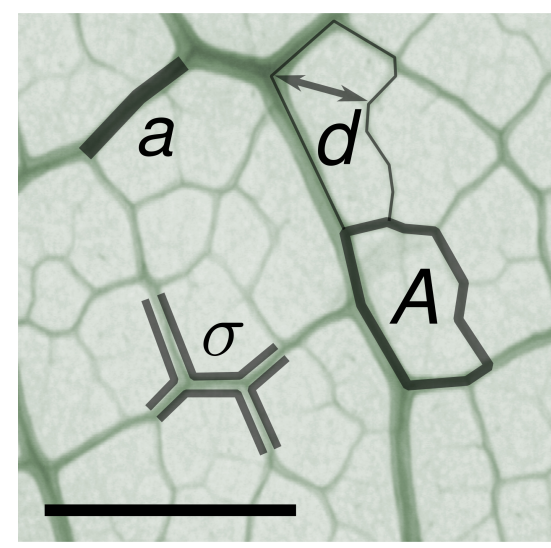

FIGURE 4.2: Geometric leaf traits schematically overlaid on top of an image of the vein network in one leaflet of Protium grandifolium. The traits shown are areole area $A$, distance between adjacent veins $d$, vein density (total length of venation per area) $\sigma$ and vein diameter a. We additionally consider (not shown) areole density (number of areoles per area) $\rho_{A}$ and vein radius weighted by length of venation between two junctions $a_{\ell}$. The scale bar is $1 \mathrm{~mm}$. (Redrawn and extended after [62]). all $d_{i}$.

\subsubsection{Areole area}

Mean areole area $A$ is calculated by taking the facets of the network and calculating their areas using the standard formula for the area of a general polygon. The formula for a single areole area $A_{i}$ is

$$
A_{i}=\frac{1}{2}\left|\sum_{k=0}^{N-1}\left(x_{k} y_{k+1}-x_{k+1} y_{k}\right)\right| .
$$

Here, the sum runs over all $N$ vertices $\left(x_{k}, y_{k}\right)$ in the closed polygon, i.e., the first and last coordinate pairs are identical. Then, the mean over the $A_{i}$ is taken. 


\subsubsection{Areole density}

Areole density $\rho_{A}$ is simply the number of areoles divided by the total area of the leaf or leaf segment,

$$
\rho_{A}=\frac{\text { total number of areoles }}{\text { total area }} .
$$

\subsubsection{Weighted vein thickness}

Weighted vein thickness $a_{\ell}$ is calculated as the average

$$
a_{\ell}=\frac{1}{w} \sum_{j} a_{j} \ell_{j}, \quad w=\sum_{j} \ell_{j},
$$

where $a_{j}$ are the diameters of individual veins (i.e., straight venation segments between two junctions), and $\ell_{j}$ are their lengths. We chose this weighting to prevent over-representation of very short segments that are sometimes spurious.

\subsection{Topological leaf traits}

In this section, we describe the hierarchical decomposition algorithm and show how to use the resulting nesting tree to extract a measure of the quantitative hierarchical topology of nested loops in vein networks. Additionally, we show how the topological length of tapered veins can be used to complement loop nesting (see Figure 4.3).

\subsubsection{Hierarchical decomposition}

The hierarchical decomposition algorithm itself is rather straightforward (see Figure 4.3 (a) and Figure 4.6). However, given a weighted

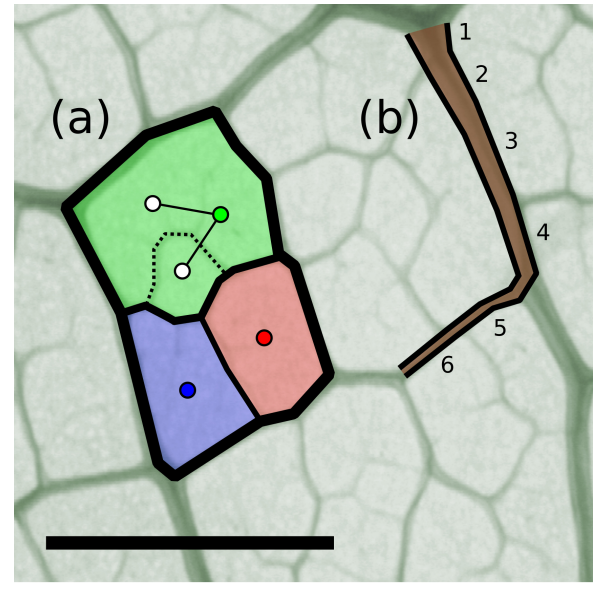

FIGURE 4.3: Topological leaf traits schematically overlaid on top of an image of the vein network in one leaflet of Protium grandifolium (the same as in Figure 4.2). (a) Hierarchically nested structure as measured by the nesting tree. Two areoles (represented by white dots) with the smallest width intersection (dotted line) are merged to form a larger facet (represented by green dot), and the intersection is removed. The white dots are connected to the green dot, forming the first part of the nesting tree. The algorithm is presented in detail in Section 4.3.1. (b) Topological vein tapering. Starting from an edge, we walk on the network to that adjacent edge with the largest diameter that is still smaller than that of the current edge. The scale bar is $1 \mathrm{~mm}$. graph representing the venation network of a leaf (or even other planar networks), several pre-processing steps need to be applied first to convert a general network from our data set into a planar polygon which can be decomposed. Because the vectorization procedure we use for our leaf images can produce errors and artifacts such as spurious crossing edges, we take some heuristic measures to ensure planarity. 

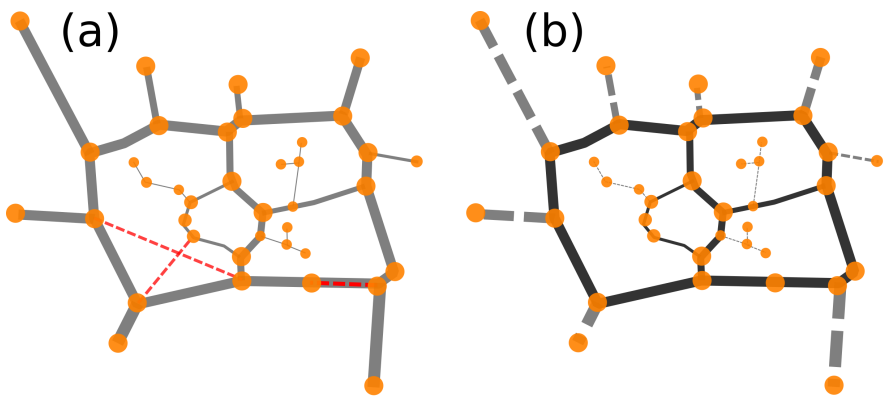

(c)

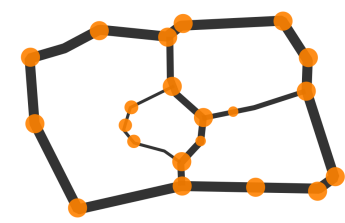

FIGURE 4.4: Illustration of the pre-processing steps. (a) Edge crossings and collinear edges (red dashed lines) are detected and removed. (b) Treelike components (dashed edges) are detected and removed. (c) The resulting planar polygon represents the "loopy backbone" $G$ of the network.

We use the general rule to remove rather than try to fix "broken" parts of a network. As long as the excised regions are small enough, this should not bias the estimates of our metrics too strongly. The basic idea is to remove errors from the network and prune treelike components, such that we are left with the "loopy backbone" G of the network (see Figure 4.4). This loopy backbone is then used to detect the fundamental cycles of the network (the facets of the planar polygon) that are used for hierarchical decomposition. For a detailed description of the vectorization process, the pre-processing steps, and an algorithm to compute the fundamental cycle basis, see Chapter B of the Appendix.

We now describe the hierarchical decomposition algorithm. We split up the procedure into sub-algorithms for the construction of the cycle dual graph, and then the extraction of the nesting tree.

\subsubsection{Constructing the cycle dual graph}

The cycle dual graph $G^{*}$ is constructed to represent neighborhood relationships between the fundamental cycles. An efficient algorithm to achieve this proceeds by iterating through all cycles, storing for each edge in the cycle which cycle(s) it belongs to. This way, one can obtain for each edge a pair of cycles that the edge belongs to by inverting the obtained relationship because each edge can belong to at most two cycles in a planar graph. This information be used to construct the cycle dual graph $G^{*}$ in which each node corresponds to one cycle, and cycles that share at least one edge are connected. Finally, the outer boundary cycle is removed, and each cycle sharing edges with the boundary is assigned its own "virtual" boundary cycle

(a)

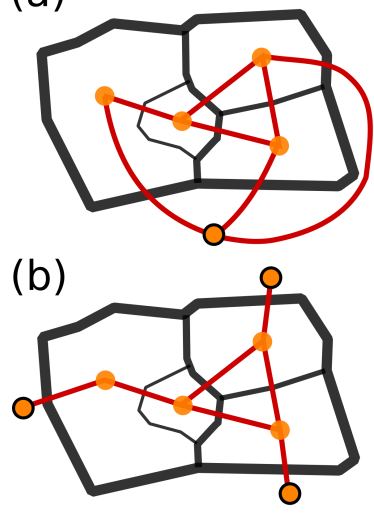

FIGURE 4.5: Dual graphs used for hierarchical decomposition. The graph-theoretical dual (a) is turned into the cycle dual (b) by introducing "virtual" boundary nodes. (replacing the single boundary cycle). This way, more information about the cycles on the network boundary is retained. The resulting graph is very similar to the dual from Chapter D in the appendix, except that self-loops represent- 

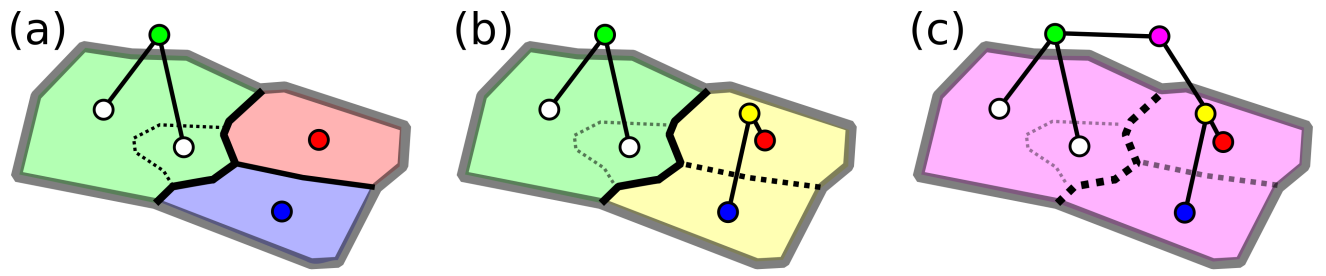

FIGURE 4.6: Example hierarchical decomposition. (a-c) Successive detection of smallest intersection between cycles and construction of the nesting tree on top of the original graph. Nesting tree nodes have the same color as the graph cycles they represent.

ing boundaries are replaced by connections to "virtual" boundary loops. The procedure effectively first constructs Figure 4.5 (a) and turns it into Figure 4.5 (b).

\subsubsection{Hierarchical decomposition}

The hierarchical decomposition algorithm proceeds as follows. We start with the cycle dual graph $G^{*}$ and a set of disconnected, single nodes $S=\left\{c_{i}\right\}$ representing the fundamental facets that will eventually be leaf nodes of the nesting tree $T$. At each step,

1. find a pair of facets $c_{i}, c_{j}$ from $S$ such that $\min _{e \in c_{i} \cap c_{j}} a_{e}$ is minimal over all pairs of adjacent cycles, where $a_{e}$ is the edge diameter. Effectively, we find the two cycles sharing the minimum diameter edge.

2. construct the cycle $c_{k}=c_{i} \triangle c_{j}$, where $\triangle$ denotes set-theoretic symmetric difference on the set of edges. The cycle $c_{k}$ is the facet formed by adding facets $c_{i}$ and $c_{j}$ and removing their intersection.

3. add a new node $c_{k}$ to the tree $T$ and connect it to the original cycles using the new edges $\left(c_{i}, c_{k}\right),\left(c_{j}, c_{k}\right)$.

4. remove the nodes $c_{i}, c_{j}$ from $G^{*}$ and add $c_{k}$ instead. Ensure that all neighboring cycles of $c_{i}$ and $c_{j}$ are neighbors of $c_{k}$.

The steps are repeated only one cycle remains in $G^{*}$. The result of this algorithm is a binary tree graph $T$, called the hierarchical decomposition tree or nesting tree, representing the nesting structure of loops within the original graph $G$. An example is shown in Figure 4.6. We note that the map $G \mapsto T$ is not injective. Many inequivalent planar graphs map to the same nesting tree.

\subsubsection{Quantifying the nesting tree}

The hierarchical decomposition algorithm produces a nesting tree which encodes the hierarchical nesting of loops in the planar network. Although binary trees are highly constrained combinatorial structures, they still have measurable degrees of freedom which can be used to extract information about the underlying nesting 
structure. In neuroscience, binary trees have been used extensively as models for dendritic growth of neurons [133], and several methods for quantifying their topology were developed $[129,130,134]$. The metric found to be most highly discriminative between different topologies in a statistical sense was shown to be the one described in [130], and in the following, we will use a variant of this (the nesting number) to quantify the nesting tree structure. We note that other metrics have been proposed to quantify binary trees. ${ }^{1}$

Another metric inspired from complex network science is the subtreedegree distribution of the nesting tree. It is defined in a similar way to the degree distribution for complex networks (see [139]) but instead captures the size distribution of subtrees. It has been applied to the characterization of urban street grids, exhibiting power law tails [140]. However, we do not use this metric because estimating it automatically in a reliable fashion tends to be difficult due to the fitting procedures involved. We now describe the nesting number.

Each node $j$ in the nesting tree can

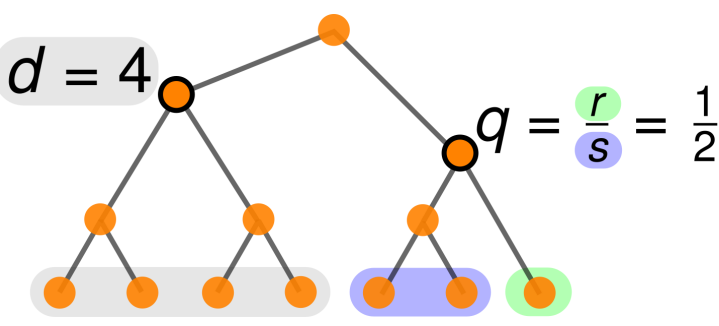

FIGURE 4.7: Properties of the binary nesting tree. Each node is assigned a subtree degree $d$, the number of leaf nodes in subtree rooted at the node, and a partition ratio $q=\frac{r}{s}$, the ratio of the left and right subtree degrees that have been ordered such that $s \geq r$. The (weighted) average partition ratio is called the (weighted) nesting number, providing a summary statistic of the binary tree properties. be assigned a subtree degree $d_{j}$, the number of leaf nodes in the subtree rooted at $j$. For nodes with $d_{j}>1$, we define the left and right subtree degrees $s_{j}, r_{j}$ as the degrees of the subtrees rooted at the left and right child nodes. We order the nodes such that $r_{j} \geq s_{j}$. It is now sensible to consider the partition ratio

$$
q_{j}=\frac{s_{j}}{r_{j}}
$$

measuring how the leaf nodes are distributed to the left and right subtrees. As a summary statistic, one can look at the nesting number

$$
Q=\frac{\sum_{j} w_{j} q_{j}}{\sum_{j} w_{j}}
$$

the weighted mean of all nesting ratios. Sensible choices of weights are $w_{j}=1$, resulting in a simple average (we call this the unweighted nesting number $Q_{u}$ ), and $w_{j}=d_{j}-1$, the degree-weighted nesting number $Q_{w}$.

Since the number of nodes at level $n$ (distance from the root) tends to grow like $2^{n}$ in a fairly balanced tree, the unweighted nesting number $Q_{u}$ will be dominated

\footnotetext{
${ }^{1}$ Most well-known are the Horton-Strahler number [135, 136] and the Shreve number [137] which were introduced to quantify the geomorphology of rivers. However, they are to a large degree uninformative when it comes to detecting structure or randomness [138]. Therefore, we will not use them in this work.
} 
by the contribution of nodes far away from the root. Theses contributions correspond to local features at a high level of the hierarchy. In contrast, $Q_{w}$ corrects for this by giving nodes in the upper level of hierarchy a very large weight, such that the local features become negligible in comparison, and $Q_{w}$ is dominated by global features of the hierarchy.

\subsubsection{Statistical distance between topologies}

It is an interesting problem to quantify how similar (or different) two nesting trees are in a statistical sense. This allows us to make quantitative statements about topological similarity of networks in contrast to qualitative, subjective statements. The problem of assigning a distance measure between binary trees has some history in the mathematics of botany and phylogeny. One approach has the tree represented as a point in a space of nontrivial curvature and then calculate geodesics [141], another defines a tree-edit distance, counting the minimum number of node insertions, removals, and re-labelings necessary to turn one tree into the other $[142,143]$. Though elegant, both approaches suffer from problems. For the curved-space method, nodes need a label and between two trees to compare, the leaf nodes must be identical, which is natural for phylogenies but impossible for leaves. The edit-distance is dominated by the effect of size differences as soon as two trees differ in their number of nodes appreciably. Again, this is common in nesting trees for different leaves.

Thus, we decide to employ a different measure which is based on a distance between the statistical distributions of nesting ratios $q_{j}$. Let $F_{1,2}(q)$ be the empirical cumulative distribution functions of nesting ratios for two nesting trees. Then we define the Kolmogorov-Smirnov distance

$$
D_{\mathrm{KS}}=\max _{q}\left|F_{1}(q)-F_{2}(q)\right| \text {. }
$$

The distance (4.7) is a heuristic measure that is robust when tree sizes differ and that captures statistical similarity well. Being derived from the corresponding Kolmogorov-Smirnov statistic (see Chapter A of the Appendix), it is also wellunderstood.

\subsubsection{Topological tapering length}

Tapering is a hallmark of plant venation, and can be observed both in flat leaves (see Figure 4.3 (b)) and needles (see Chapter 9 of Part III), where it is responsible for efficient transport $[45,71]$. Because of the two-dimensional, complex network-like nature of leaf venation, results similar to those found for needles, i.e., measuring tapering of the conduits themselves, are difficult to obtain. Instead, we opt for a topological characterization of tapering, the topological tapering length. We define this topological length for an edge $e$ by the following algorithm. 
1. Starting from the current edge $e$, find all adjacent edges $\left\{f_{i}\right\}$ with diameter less than the diameter of $e$.

2. Find the edge $f$ with maximum diameter among the $\left\{f_{i}\right\}$.

3. Set the current edge to $f$.

These steps are repeated until no more edges with smaller diameter can be found. The topological tapering length $L_{e}$ is then defined as the number of edges traversed in this fashion, starting from $e$. For a full network or network fragment, it is sensible to define the mean topological length

$$
L_{\text {top }}=\frac{1}{|E|} \sum_{e \in E} L_{e}
$$

as a measure of overall tapering. Because in our data set, some nodes with degree 2 exist that would distort this metric (each edge can in principle be subdivided an arbitrary number of times, changing the associated tapering length), these nodes are removed and the associated edges merged.

\subsubsection{Topological length and nesting number}

Topological tapering length and nesting tree metrics encode complementary features of the weighted network topology. Large nesting numbers correspond to a highly nested structure, large topological lengths correspond to a large amount of long range tapering. Small values of either metric essentially correspond to a random distribution of edge diameters. These features can be present or absent independently from each other (see also Figure 4.8), thus furnishing two complementary metrics.

It should be noted that in order to calculate the different metrics, only the network topology and the relative ranks in terms of diameter of the edges are strictly necessary, not the exact values of edge diameters. Thus, the metrics should be expected to be robust against noise in the data. In practice, the nesting number is very robust even when a part of the smallest veins are pruned away. The topological tapering length on the other hand is more sensitive
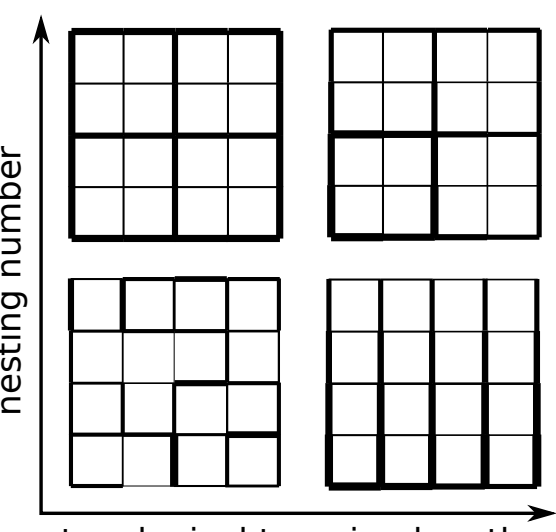

topological tapering length

FIGURE 4.8: Topological tapering length and nesting number encode complementary information about network topology. High values of the tapering length correspond to long, tapered venation structures, high values of the nesting number correspond to a large amount of hierarchical order. to noise. This will be seen explicitly in the later chapters. 



\section{Results}

In this chapter we collect and present the analysis results of our leaf data set. The data set consists of 207 different vouchered leaves and leaflets ${ }^{1}$ from diverse species of dicotyledonous plants mainly from the Burseraceae family. Even though most of the species represented are closely related, the leaf networks present a striking diversity of form. Before further analysis, all leaves containing fewer than 256 areoles were discarded because they are either too small to be able to provide meaningful statistics or come from damaged samples. This reduced data set contains 186 good quality specimens representing 137 different species.

We present the results in two separate sections. First, we analyze the complete leaf venation networks, calculating averages of the various geometric and topological metrics described in Chapter 4 for each whole network. The data is then further analyzed by investigating its principal components and latent factors. Leaves with similar topology are identified according to a statistical distance between nesting ratio distributions. The calculated distances are also used to perform hierarchical clustering, showing a tendency to cluster specimens of the same species and separating the Burseraceae family from the other families to some degree. However, detailed phylogeny is not represented.

Second, we split each leaf network into fragments in-silico of equal size, computing the metrics for each fragment. We then proceed to employ discriminant analysis to compare how well a given leaf specimen can be identified from its fragments, showing that using topology in addition to standard geometry provides a highly significant improvement.

It should be noted that we did not try to design a "production quality" leaf identification system but rather strove to create a proof-of-concept that topological information can be highly relevant in such tasks.

In Chapter 6, we move from the purely descriptive analysis of this chapter to an attempt at explaining the observed data in terms of a simple, phenomenological model of venation growth.

\subsection{Full leaf networks}

In this section we show the analysis results for the full leaf networks. For each leaf specimen in the reduced data set, the geometric and topological metrics were calculated. In order to minimize effects from different size leaves, the nesting ratios were taken only from those subtrees of the nesting tree with degree $d \leq 256$. These were used to calculate the two averaged nesting numbers $Q_{u, w}$ as well as

\footnotetext{
${ }^{1}$ Vouchered specimens are representative for a given species and are typically collected as part of an ecological survey.
} 

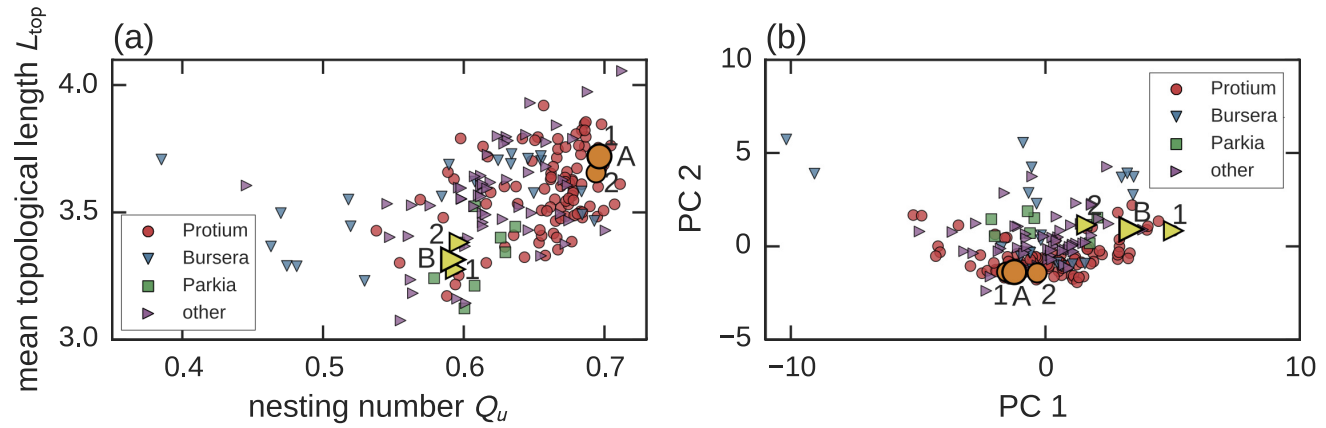

FIGURE 5.1: Topological degrees of freedom and projection on the first two principal components. We mark two test leaves (orange circle, A, Protium grandifolium; yellow triangle, B, Dalbergia miscolobium) together with their two nearest neighbors $(1,2)$ according to the statistical KS distance between nesting ratios. (a) Plot of the data projected onto the topological degrees of freedom $Q_{u}, L_{\text {top }}$. The test leaves' two nearest KS neighbors are also very close in their means $Q_{u}$, and reasonably close in $L_{\text {top. }}$. We explicitly marked the three most abundant genera in the data set, revealing a weak clustering trend. (b) Plot of the data projected onto the first two principal components. The test leaves' two nearest neighbors are very close in PC 2 (topology) and also reasonably close in PC 1 (geometry).

\begin{tabular}{rrrrrrrrr|r}
\hline & $L_{\text {top }}$ & $Q_{u}$ & $Q_{w}$ & $\sigma$ & $a$ & $A$ & $\rho_{A}$ & $d$ & rel. expl. var. \\
\hline PC 1 & -0.29 & -0.15 & -0.14 & 0.45 & -0.44 & -0.36 & 0.40 & -0.43 & $55.9 \%$ \\
PC 2 & -0.29 & -0.61 & -0.64 & -0.04 & 0.19 & 0.30 & -0.04 & 0.09 & $26.2 \%$ \\
PC 3 & -0.54 & 0.36 & 0.16 & 0.20 & 0.23 & 0.42 & 0.47 & 0.25 & $9.3 \%$ \\
PC 4 & -0.71 & 0.10 & 0.07 & -0.31 & -0.14 & -0.39 & -0.44 & 0.15 & $5.5 \%$ \\
\hline
\end{tabular}

TABLE 5.1: First four eigenvectors of the covariance matrix (principal components) as well as the relative explained variance. The first principal component is dominated by geometry whereas the second is dominated by topology. Topological length is shared equally between the two. The other components do not have a clear interpretation.

the nesting ratio statistics used to define topological distance between specimens. This means we calculated local features of the venation system. The scale on which these features occur is approximately $7 \mathrm{~mm}$.

Because the different metrics (different features) have different units, we rescaled the reduced data set to zero mean and unit variance in each feature before performing further data analysis, rendering them comparable. Figure 5.1 (a) shows the topological degrees of freedom in the data set. We marked two test leaves together with their two nearest neighbors according to the KS statistical distance. The test leaves are shown in Figure 5.4 together with their closest neighbors according to our statistical distance. Additionally, we marked the members of the three most abundant genera in our data set. They are 98 Protium, 21 Bursera, and 8 Parkia. There appears to be a weak trend towards members of the same genus clustering, but our data set is inadequate to draw firm conclusions at this stage. 

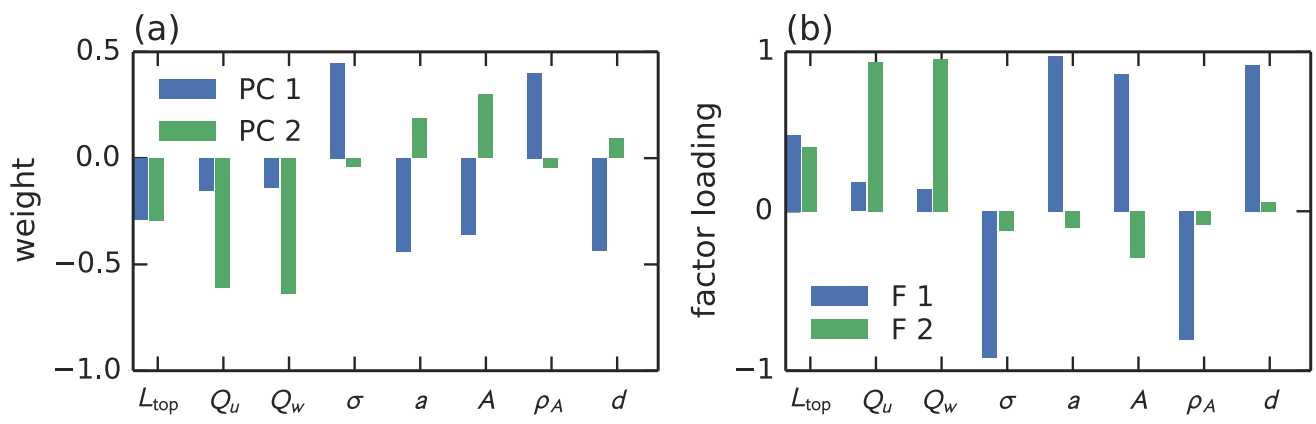

FIGURE 5.2: The first two principal component weights and the first two latent factors loadings for the eight topological and geometrical leaf features. (a) Principal component weights. (b) Factor loadings. Both show very similar trends, the first component (factor) being dominated by geometry and the second by topology. Mean topological length appears to be shared equally between the two.

\begin{tabular}{lrrrrrrrr|r}
\hline & $L_{\text {top }}$ & $Q_{u}$ & $Q_{w}$ & $\sigma$ & $a$ & $A$ & $\rho_{A}$ & $d$ & rel. expl. var. \\
\hline F 1 & 0.48 & 0.18 & 0.14 & -0.92 & 0.97 & 0.86 & -0.81 & 0.92 & $54.0 \%$ \\
F 2 & 0.40 & 0.94 & 0.96 & -0.12 & -0.10 & -0.29 & -0.09 & 0.06 & $25.9 \%$ \\
F 3 & -0.20 & 0.20 & 0.08 & 0.32 & 0.15 & 0.37 & 0.54 & 0.05 & $8.1 \%$ \\
F 4 & -0.27 & 0.09 & -0.13 & -0.06 & -0.00 & -0.06 & 0.08 & 0.21 & $2.0 \%$ \\
\hline rel. noise var. & $6.2 \%$ & $0.5 \%$ & $0.6 \%$ & $0.4 \%$ & $0.2 \%$ & $0.4 \%$ & $0.5 \%$ & $1.2 \%$ & \\
\hline
\end{tabular}

TABLE 5.2: Factor loading matrix entries for the leaf data set as well as estimated noise variances for factor analysis with four factors. Results for the factor loadings and explained variance are comparable to those of PCA (see Table 5.1). Relative noise variance and relative explained variance add up to $100 \%$.

\subsubsection{Principal Component Analysis}

The results of principal component analysis of the reduced full leaf data set are shown in Figure 5.1 (b) (projection onto the first two components) and Table 5.1. We find that the first principal component is dominated by geometric leaf network features, whereas the second is dominated by topological features. The topological lengths $L_{\text {top }}$ are roughly shared equally between the first two components. The first two principal component vectors are visualized in Figure 5.2 (a). We interpret this as a signature of two orthogonal ${ }^{2}$ directions in the phenotypic space of leaf venation (our feature space), one spanned primarily by leaf geometry, the other primarily by leaf topology.

\subsubsection{Factor Analysis}

A latent factor model with 4 factors was fit to the reduced full leaf data set. This is the maximum number of factors compatible with the feature space, with maximum $p$-value among all possible factor models. Even though $p=0.003$ (see Section A.4.2 for an explanation), the model still provides a good interpretation of the data. The results are shown in Figure 5.2 (b) and Table 5.2. They are

\footnotetext{
${ }^{2}$ In the sense of PCA.
} 

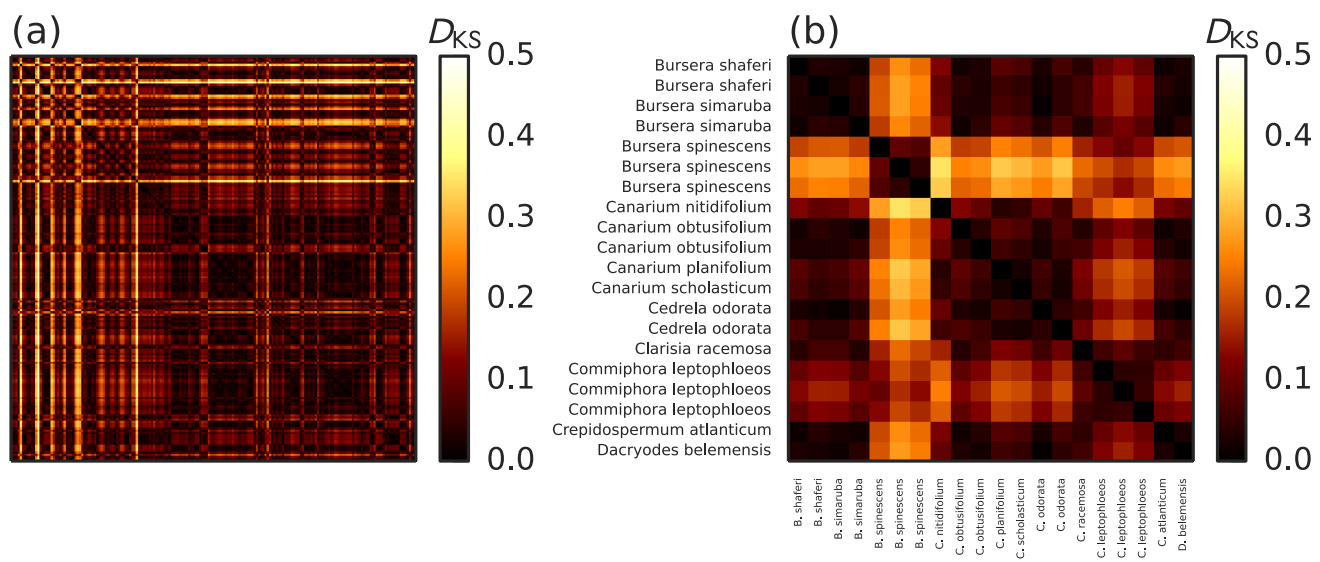

FIGURE 5.3: Topological similarity as measured by the KS distance between nesting ratio distributions. (a) Distance matrix between all analyzed 186 specimens. The matrix is ordered lexicographically by species name. (b) A small submatrix showing the relationships between 20 specimens. Identical species tend to be close but there do not appear to be clusters of significantly different hierarchical topology.

comparable to the results from PCA because again, the first factor is dominated by geometric features, whereas the second is dominated by topology, with the topological lengths contributing approximately equally to either.

\subsubsection{Topological similarity}

We quantify the topological similarity of leaves using a distance measure between the nesting ratio statistics (thresholded at $d \leq 256$ ) between leaves. We tested several statistical distances and found that the Kolmogorov-Smirnov distance $D_{\mathrm{KS}}=\max _{x}\left|F_{1}(x)-F_{2}(x)\right|$ worked best. ${ }^{3}$ In Figure 5.3, we show the distance matrix computed from pairwise KS distances between thresholded nesting ratio statistics of all 186 specimens. The structure of the distance matrix is such that specimens belonging to the same species tend to be close, but there do not appear to be clusters of significantly differing topology.

The data set therefore appears as a continuum of varying topologies with little internal structure or clustering. This may likely be due to the close relatedness of the species involved but could also point towards a universal mechanism during vein morphogenesis which leads to very similar vein topologies. We explore this idea further in the next chapter.

\subsubsection{Hierarchical clustering}

Hierarchical clustering was employed to further try to uncover similarities between groups of leaf network specimens based on topology. The completelinkage method (see Section A.5.3) was applied to the distance matrix $D_{\mathrm{KS}}$ to

\footnotetext{
${ }^{3}$ Other tested distances include the Jensen-Shannon divergence and the Bhattacharyya distance (see Section A.2.2 in the appendix).
} 

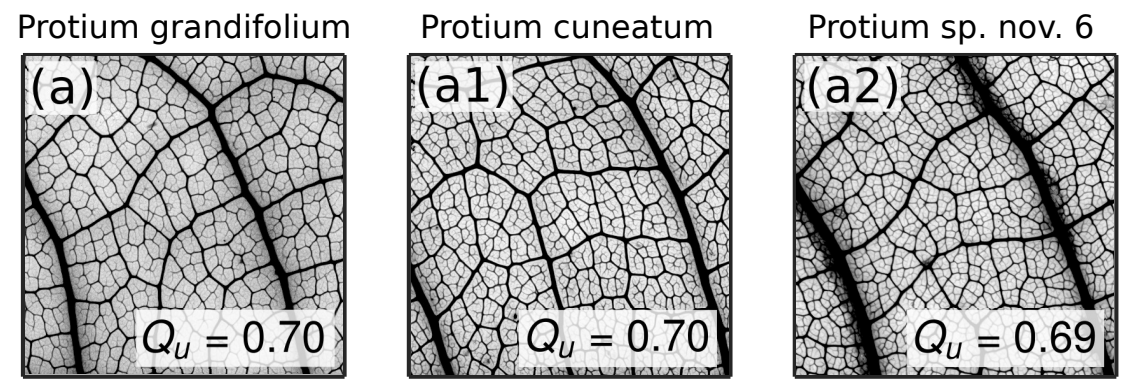

Dalbergia miscolobium

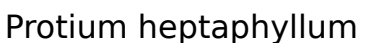

Parkia nitida
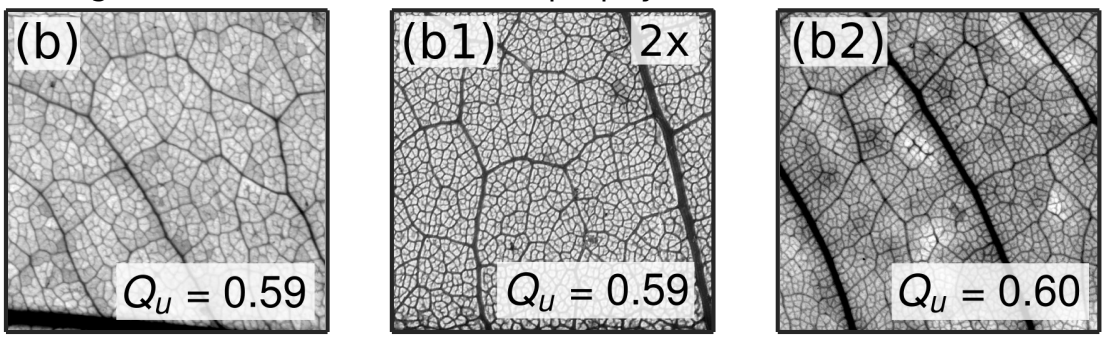

FIGURE 5.4: Two test leaves (the same as in Figure 5.1) together with their two nearest neighbors according to $D_{\mathrm{KS}}$ applied to the nesting ratio statistics. Shown are $1 \mathrm{~cm} \times 1 \mathrm{~cm}$ grayscaled crops of the original scans. The crop (b1) was zoomed in by a factor of 2 because of the very dense venation. (a) The two nearest topological neighbors $(\mathrm{a} 1,2)$ show high similarity to the test leaf. All leaves have a high nesting number $Q_{u}$. (b) The two nearest topological neighbors $(\mathrm{b} 1,2)$ show reasonable similarity to the test leaf. All leaves have a low nesting number $Q_{u}$.

produce the dendrogram in Figure 5.5. This method was chosen because it tends to produce clusters of approximately equal size and is not prone to the "chaining" effect [144].

Inspection of the dendrogram reveals that topology alone indeed clusters specimens belonging to the same species close together. However, comparison with phylogenetic trees derived from molecular genetic methods [145, 146] shows that topology alone does not appear to reflect phylogeny. A more sensitive phenotypic tool might uncover additional correlations, but it is possible that topology alone is insufficient to describe phylogenetic relationships.

We performed a similar analysis on a distance matrix computed from the cosine distances of normalized feature vectors (the same as in Section 5.1.1). Although the distances between same species appear smaller and those between different species larger, the clustering results do not appear to change significantly.

\subsection{Leaf network fragments}

In this section we present the results of the leaf fragment analysis. Each leaf was split in-silico into $1.2 \mathrm{~cm} \times 1.2 \mathrm{~cm}(3000 \mathrm{px} \times 3000 \mathrm{px})$ fragments, then the local geometric and topological features were calculated for each fragment individually. 


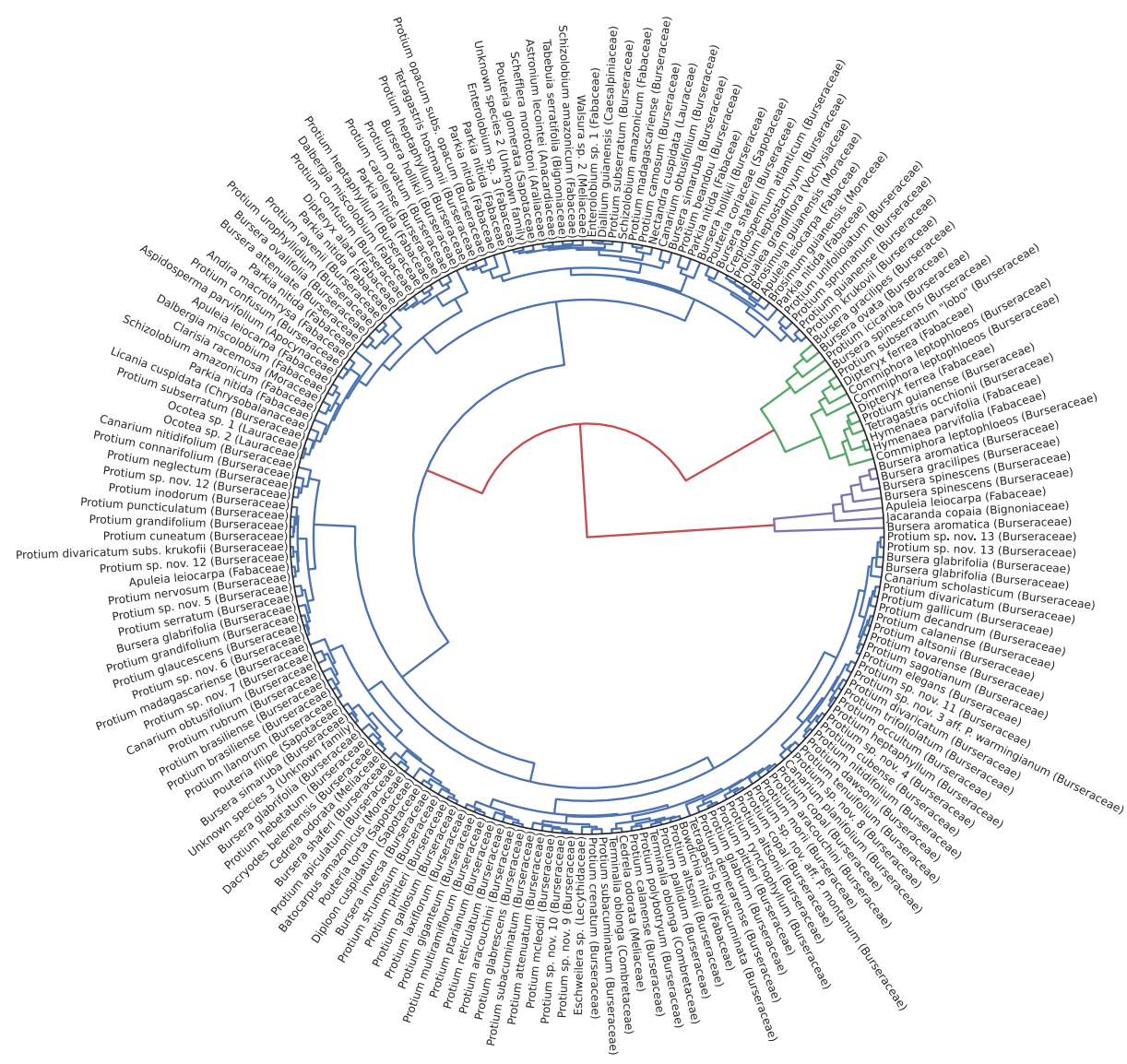

FIGURE 5.5: Hierarchical clustering of reduced full leaf data set. The dendrogram was produced using the KS distances between nesting ratio statistics and the complete-linkage (maximum distance) clustering method. The height of the U-shaped links corresponds to the distance between clusters, different colors were used when the distance between clusters was larger than 0.5 times the maximum distance. Specimen belonging to the same species tend to be clustered closely together, but there appears to be no correlation with phylogeny.

In order to estimate the usefulness of our new topological features, we test how well the features allow one to discriminate between different leaf specimens or species (or identify a particular specimen or species) first based on geometry alone, then on geometry combined with topology. We find a significant improvement of identification accuracy when using topology together with geometry. To this end, we employ the Machine Learning technique of Linear Discriminant Analysis (see Chapter A of the Appendix), which fits a hyperplane to a training subset of our data that optimally separates known specimens or species. This hyperplane is then used to predict specimen or species of a test subset of our data, and the prediction accuracy and receiver operating characteristic are measured. 


\subsubsection{Linear Discriminant Analysis}
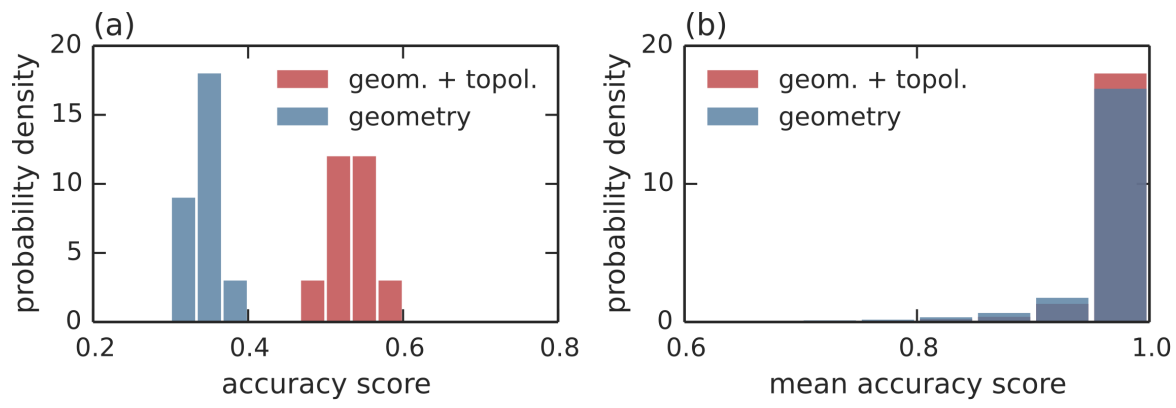

FIgURE 5.6: Accuracy estimation of Linear Discriminant Analysis for leaf specimen fragments. (a) Histograms of the distribution of accuracy scores obtained using 10-fold stratified cross-validation fitting LDA to all fragments. Combining geometrical and topological information improves the classification accuracy by roughly $54 \%$ from 0.35 (95\% CI: $[0.31,0.39])$ to 0.54 (95\% CI: $[0.48,0.60])$. The improvement is significant (Welch's $t(15.6)=15.8, p<0.001)$. (b) Histogram of pairwise Linear Discriminant Analysis mean accuracy score distribution. For each pair of specimens, LDA was fitted to the fragments and mean accuracy of the estimator was calculated using cross validation. Topology improves the mean scores significantly in distribution (KS $D=0.07, p<0.001)$.
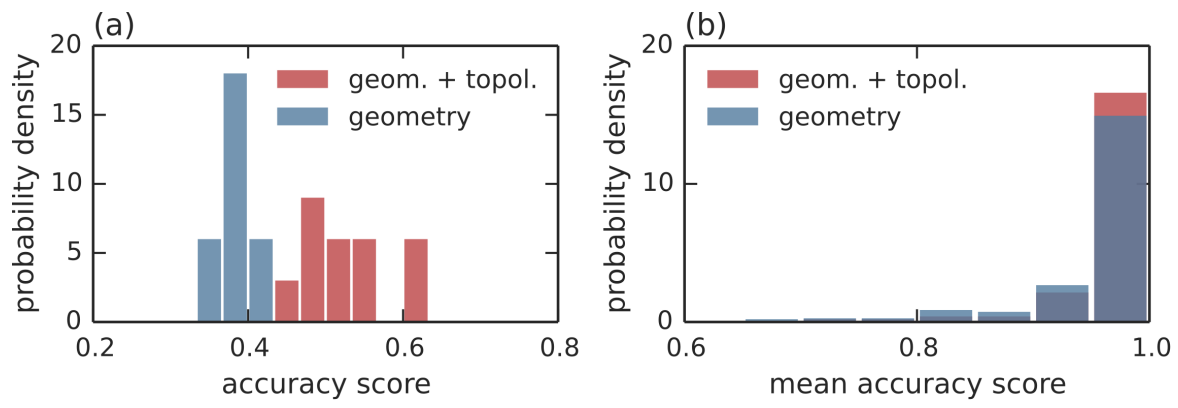

FIGURE 5.7: Accuracy estimation of Linear Discriminant Analysis for leaf species fragments. (a) Histograms of the distribution of accuracy scores obtained using 10-fold stratified cross-validation fitting LDA to all fragments. Combining geometrical and topological information improves the classification accuracy by roughly $37 \%$ from 0.38 (95\% CI: $[0.33,0.43])$ to 0.52 (95\% CI: $[0.42,0.63])$. The improvement is significant (Welch's $t(12.7)=7.4, p<0.001$ ). (b) Histogram of pairwise Linear Discriminant Analysis mean accuracy score distribution. For each pair of specimens, LDA was fitted to the fragments and mean accuracy of the estimator was calculated using cross validation. Topology improves the mean scores significantly in distribution (KS $D=0.21, p<0.001)$.

We used LDA to assess the separability of leaf specimen and leaf species based on geometric and topological metrics. Each leaf graph was cut into $3000 \mathrm{px} \times$ 3000 px fragments which were analyzed separately, calculating the same metrics as for the full leaves. For all 186 leaves, fragments were generated. Fragments with fewer than 128 areoles were discarded, leaving a total data set of 3446 fragments representing 183 specimen (some leaves were too small to be included in this analysis). Reducing the data set to only those species for which more than one specimen was available, we were left with 1123 fragments representing 80 

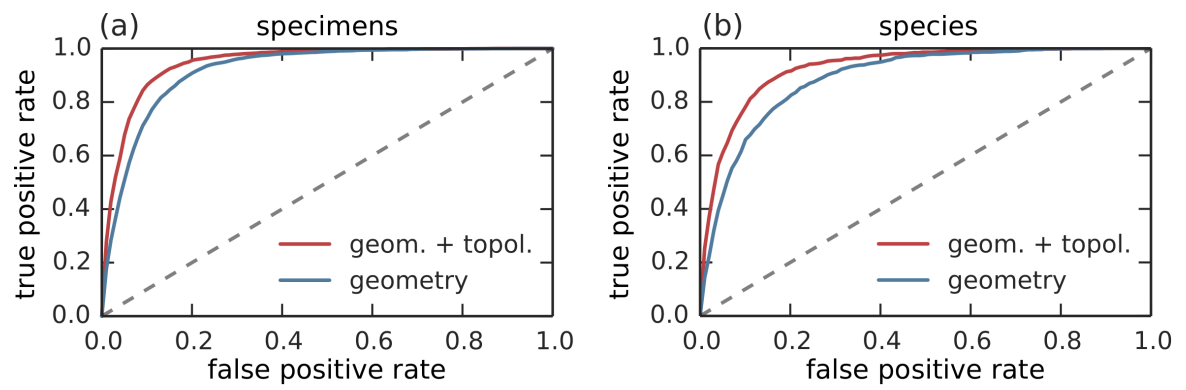

FIGURE 5.8: Micro-averaged Receiver Operating Characteristic (ROC, plotting true positive rate against false positive rate, see Chapter A of the Appendix) improves when using topological metrics. (a) Specimen ROC area-under-curve (AUC) improves from 0.92 (95\% CI: [0.88, 0.95]) to 0.95 (95\% CI: [0.91, 0.97]) using topology and geometry as compared to geometry alone. (b) Species ROC AUC improves from 0.89 (95\% CI: $[0.85,0.92])$ to 0.93 (95\% CI: $[0.90,0.95])$ using topology and geometry as compared to geometry alone.

specimens of 33 species.

Two test scenarios were considered. First, we performed LDA on the full data set, considering all fragments together with their specimen or species information in a 10-fold stratified cross-validation scheme. We then calculated the accuracy, the probability of correctly classifying a given sample, and the receiver operating characteristic given either only the geometric features or the geometric and topological features together. This was done for the specimen identification case in Figure 5.6 (a) and species identification in Figure 5.7 (a). Because we consider the entire point cloud consisting of all our data and try to separate it, this is a hard classification task.

Second, we performed a pairwise test. For each pair of leaf specimens or species, LDA was used to fit a separating hyperplane to a training subset, and then the accuracy was measured for a test subset. The pairwise test results are shown in Figure $5.6(\mathrm{~b})$ and Figure 5.7 (b). Because we only test small subsets of the data, this is a comparatively easy classification task.

In order to obtain robust estimates of the accuracies, we employed stratified cross-validation schemes (see Chapter A in the Appendix). For the species identification tests, we considered all leaf fragments from leaves of the same species as belonging to the same classification unit. We now present the detailed results.

\subsubsection{Full data set test}

In this test scenario, LDA was fit to the complete data set of leaf fragments. Because as a whole, the leaf data set forms a continuum without well-defined clusters, there is typically considerable overlap between data belonging to different leaf specimens. Therefore, this is a hard classification task, and we do not expect stellar performance in the first place, especially using a simple method such as LDA. 
Specimen identification. Using only geometrical venation traits, we found an accuracy of 0.35 (95\% CI: $[0.31,0.39])$. Adding topology, we found a significant increase to 0.54 (95\% CI: $[0.48,0.60])$ (Welch's $t(15.6)=15.8, p<0.001)$. This is a performance increase of roughly $54 \%$ when using geometry and topology, vs. geometry alone, see Figure 5.6 (a). In addition, the Receiver Operating Characteristic improves markedly, see Figure 5.8 (a).

Species identification. Using only geometrical venation traits, we found an accuracy of 0.38 (95\% CI: $[0.33,0.43])$. Adding topology, we found a significant increase to $0.52(95 \% \mathrm{CI}$ : $[0.42,0.63])$ (Welch's $t(12.7)=7.4, p<0.001)$. This is a performance increase of roughly $37 \%$, see Figure 5.7 (a). In addition, the receiver operating characteristic improves markedly as well, see Figure 5.8 (b).

\subsubsection{Pair test}

Unlike in the previous section, we now do not consider the data set as a whole but instead pick out all pairs of leaf specimens or species and calculate the cross-validated identification accuracy using LDA. Because for some leaves there were fewer than 10 fragments, we chose a $k$-fold cross-validation scheme with $k=\min \left(n_{a}, n_{b}\right)-1$, where $n_{a, b}$ is the number of fragments in specimen/species $a, b$. In comparison to the previous section, this is an "easy" classification task, and indeed accuracies tend to be high.

For most pairs the change in identification accuracy is not significant individually. However, in distribution (taking all pairs together), the improvement is significant as shown by the KS test. (see Figure 5.6 (b) for the specimen results and Figure 5.7 (b) for the species results).

\subsection{Summary}

We analyzed the phenotypic space of leaf venation networks. The features (phenotypes) investigated were a combination of traditional geometric leaf traits and novel topological traits. Principal Component Analysis and Factor Analysis revealed the topological traits as approximately orthogonal with respect to geometry, therefore constituting an entirely new dimension in the phenotypic space of leaf venation.

Quantifying topological similarity by a statistical distance and hierarchical clustering revealed that leaves from the same species indeed tend to be close in topological space. However, topology appears not sufficient to reproduce phylogenetic relationships. Similar results were obtained when using hierarchical clustering on feature vectors.

We showed that nevertheless, topological information may be highly useful for tasks such as leaf identification from fragments by splitting leaf networks into small pieces and using Linear Discriminant Analysis with and without topological features to distinguish between either specimen or species. In both 
5 Results

cases, we see a highly significant improvement in identification accuracy when using topological features in addition to geometric features. 


\title{
6 Empirical Growth Model
}

\author{
In past times when one lived in contact with nature, abstraction was \\ easy; it was done unconsciously. Now in our denaturalized age \\ abstraction becomes an effort.
}

(Piet Mondrian)

In this chapter, provide a tentative explanation for the data observed in Chapter 5 by developing an empirical model for vein topology morphogenesis that takes into account sources of randomness during development. It is compatible with both competing theories of higher order vein morphogenesis that invoke either auxin canalization $[84,87,99]$ (some aspects of which we will investigate in Part III), mechanical instabilities $[93,94]$ or a combination of both. There is much debate about the precise details of vein morphogenesis because of the inherent difficulty in measuring either mechanical stress or auxin gradients in the developing leaf.

Our model is agnostic to such problems because it abstracts from the underlying mechanism and simply stipulates that vein loops are subdivided roughly equally by new veins as the leaf grows. Thus, it tries to capture the essential features of loop formation leading to specific, measurable topological features while remaining blind with regard to the underlying biology. Still, the process our model intends to capture is empirically very well known [86, 147]. It should be noted that low order vein formation (main and secondary veins) can not be explained using our model because the growth rules are symmetric (there is no preferred direction for a vein to develop along). Low order veins require an explicit symmetry breaking mechanism (in auxin canalization models, this is provided by the presence of a sink at the petiole, see Chapter 10 . Mechanical models can also incorporate this aspect [92]). Similar models have been considered before but were mostly applied to the study of general network geometry or as toy models $[93,148]$. We stress again that our model does not aim to exactly reproduce vein morphogenesis in any way but only to serve as an investigation of possible effects leading to the observed topological statistics.

In our model, several parameters can be tuned explicitly in order to produce varying topologies as measured by nesting ratio and topological length statistics. We find that the statistics observed in our leaf data set can be reasonably well reproduced using the model, the topological length statistics even exceptionally well. This suggests that the topological lengths are dominated by random effects while nesting statistics can be explained in a large part by them. 


\subsection{The model}

(a)

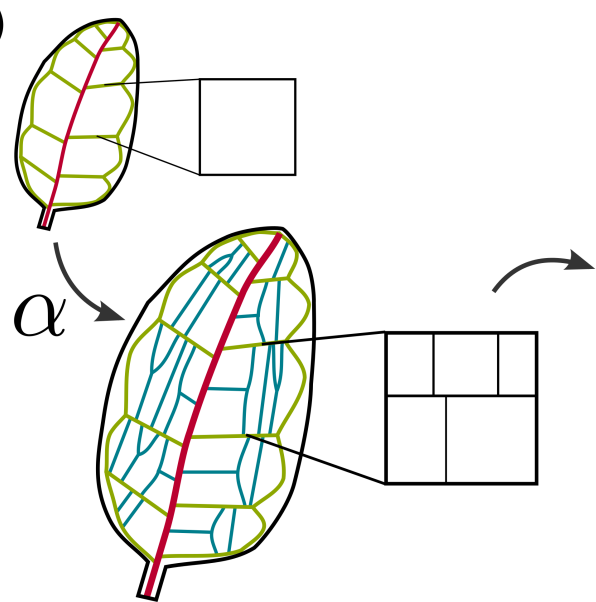

(b)

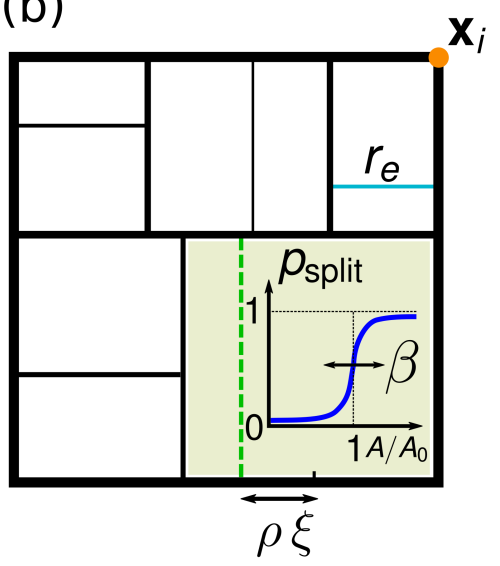

FIGURE 6.1: Sketch of the empirical growth model dynamics together with representation of leaf growth stages (compare Figure 3.3). (a) The model starts with one rectangular loop, representing one region in the leaf located inside at least a secondary vein loop. Node positions $\mathbf{x}_{i}$ grow exponentially while vein thicknesses $r_{e}$ grow linearly. The rectangular loops are successively subdivided once their area $A$ becomes close to the critical area $A_{0}$. (b) The position of a new vein is controlled by the parameter $\rho$, the relative distance from the center. The probability $p_{\text {split }}$ of forming a new vein during one time step $d t$ is a sigmoidal of width $\beta$.

Growth is modeled in phenomenological and probabilistic fashion, simulating the formation of nested loops by means of "growing rectangles which can divide." The model starts with a single rectangle. Each vertex with coordinates $\mathbf{x}_{i}$ moves according to the equation

$$
\frac{d \mathbf{x}_{i}(t)}{d t}=a \mathbf{x}_{i}(t),
$$

where $a$ is the tissue growth rate. This type of exponential growth law is popular for modeling uniform tissue dynamics in the continuum approximation [149, 150]. Here, it represents growth of the underlying tissue matrix in which the vein cells are embedded. The edges representing veins are modeled to grow linearly in width according to

$$
\frac{d r_{e}}{d t}=b .
$$

Here, $b$ is the linear thickness growth velocity and $r_{e}$ is the thickness of edge (vein) $e$.

During each time interval of length $d t$, there is a finite probability $p_{\text {split }}$ for each rectangle to instantaneously split in two by formation of a new vein. This 
probability depends on the loop area $A$ and a fixed critical area $A_{0}$ as follows:

$$
\begin{aligned}
p_{\text {split }}(A) & =\frac{w}{2}\left(1+\operatorname{Erf}\left(\frac{A-A_{0}}{\sqrt{2} \sigma_{A}}\right)\right) \\
& =w f\left(\frac{A-A_{0}}{\sigma_{A}}\right) .
\end{aligned}
$$

Here, $\sigma_{A}$ controls the width of the sigmoidal function $f$ and $w$ sets a maximum probability (the effective splitting rate). The motivation for this form is given by the fact that a single leaf tends to have roughly equally sized areoles. This can also be explained by simulation of hypothesized underlying biological mechanisms $[87,93]$. Crucially, we assume that the time scale on which veins appear is much smaller than the time scale for overall leaf growth (this is roughly justified, see Figure 2.17).

When a rectangle is divided, the position of the new edge is chosen such that it splits the rectangle along the long side. The position relative to one corner is

$$
x_{\text {rel }}=\frac{1}{2}+\rho \xi
$$

where $\xi$ is a uniform random number between $-1 / 2$ and $1 / 2$ and the parameter $\rho$ controls the strength of randomness. Due to the resulting networks' similarity to certain pieces of abstract art, they have been termed Mondrian lattices in the literature [148].

The five dimensionful parameters $a, b, A_{0}, \sigma_{A}, w$ can be combined to yield four dimensionless control parameters $\alpha=b\left(a \sqrt{A_{0}}\right)^{-1}, \beta=\sigma_{A} A_{0}^{-1}, \rho, w$. They can be interpreted as growth rate $(\alpha)$, growth noise $(\beta, \rho)$ and splitting rate $(w)$. We choose the length and time scales $x_{c}=\sqrt{A_{0}}, t_{c}=a^{-1}$, obtaining the nondimensional set of equations

$$
\begin{aligned}
\frac{d \mathbf{x}_{i}(t)}{d t} & =\mathbf{x}_{i}(t) \\
\frac{d r_{e}}{d t} & =\alpha \\
p_{\text {split }}(A) & =w f\left(\frac{A-1}{\beta}\right) .
\end{aligned}
$$

These equations can be discretized and solved using a simple forward Euler scheme. In all of the following, we choose $d t=0.01$ and $w=0.1$.

As the termination criterion we choose a final number of loops $N$ (in the following, we always arbitrarily set $N=2048$ ). After the termination criterion has been reached or exceeded (this can happen if more than one new vein is created during one time step), we add Gaussian noise with zero mean to all edge 
widths $r_{e} \cdot{ }^{1}$ The standard deviation is chosen to $f_{n} \mu_{r}$, where $\mu_{r}=\left\langle r_{e}\right\rangle$ is mean edge thickness and $f_{n}$ is a parameter. Edge widths may become negative during this procedure and such edges are removed from the network (this changes network topology). The addition of this noise is meant to represent measurement noise or intrinsic noise in the vein thicknesses. Simulations of growth conditions that produce nested vein structures but whose widths typically vary less than $f_{n} \mu_{r}$ will appear to have a more random, less highly nested topology.

We compare the simulation results to our cleared leaf data set by computing the topological features $Q_{u}$ and $L_{\text {top. }}$. In order to further constrain the model using geometric features, we also calculate for for each simulated network

$$
S_{A}=\frac{\sqrt{\frac{1}{n-1} \sum_{i}\left(A_{i}-A\right)^{2}}}{A},
$$

the standard deviation of the $n$ individual areole areas $A_{i}$ normalized by the mean areole area $A=\frac{1}{n} \sum_{i} A_{i}$. We choose this number because it is dimensionless, easy to compute and can be compared for both real and simulated leaf networks.

\subsection{Results}

We summarize the simulation results, give a general overview over the model behavior, and then compare to our cleared leaf data set. We find that most of the variation in the nesting number and indeed much of the nesting ratio statistics can be explained by the model. The topological length statistics are an even better fit, suggesting that they are in fact dominated by noise. We show this by comparing model topological lengths with $f_{n}=0$ to those with $f_{n}>0$. We further verify the model by comparing the normalized standard deviation of areole size $S_{A}$ with the data set, finding good agreement. To develop an intuition for the model, final networks for several simulation parameters are shown in Figure 6.2. Networks generated without or with very small noise parameters tend to be highly symmetric and hierarchically organized. This organization is gradually lost on all scales when increasing growth noise (i.e., $\beta, \rho$ ) and more strongly on small scales when increasing measurement noise $\left(f_{n}\right)$.

\subsubsection{Phase diagrams}

In this section, we show phase diagrams of the model behavior for varying parameters and noise levels.

The phase diagram Figure 6.3 shows the measured values of $Q_{u}$ and $L_{\text {top }}$ when varying the growth noise parameters $\beta, \rho$. At zero measurement noise $f_{n}=0$, the growth rate $\alpha$ has almost no influence on the measured values. We can

\footnotetext{
${ }^{1}$ Alternatively, noise could be added to the growth rate $\alpha$.
} 
(a)

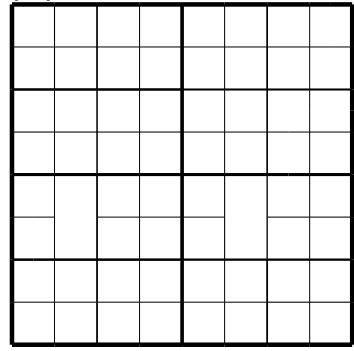

(b)

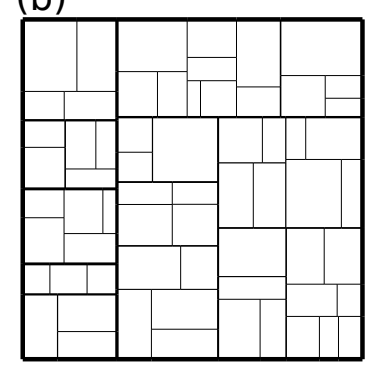

(c)

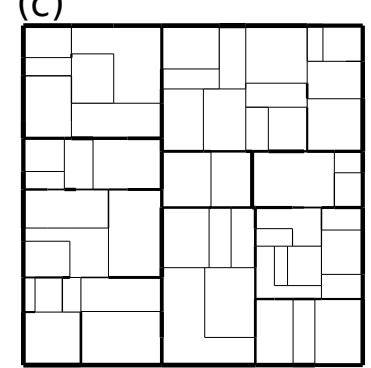

FIGURE 6.2: Hierarchical nesting of typical final networks at $N=64$. (a) No noise, $\alpha=0.5, \beta=0, \rho=$ $0, f_{n}=0$. The network is highly symmetric and hierarchically organized, $Q_{u} \approx 1$. (b) Growth noise but no measurement noise, $\alpha=0.5, \beta=0.5, \rho=0.5, f_{n}=0$. The network is much less symmetric but still shows a degree of hierarchical organization, $Q_{u}=0.79$ (c) Growth noise and measurement noise, $\alpha=0.5, \beta=0.5, \rho=0.5, f_{n}=0.2$. The network still shows some degree of hierarchical organization at the level of the thickest veins, but organization is destroyed at the level of very thin veins, $Q_{u}=0.63$.
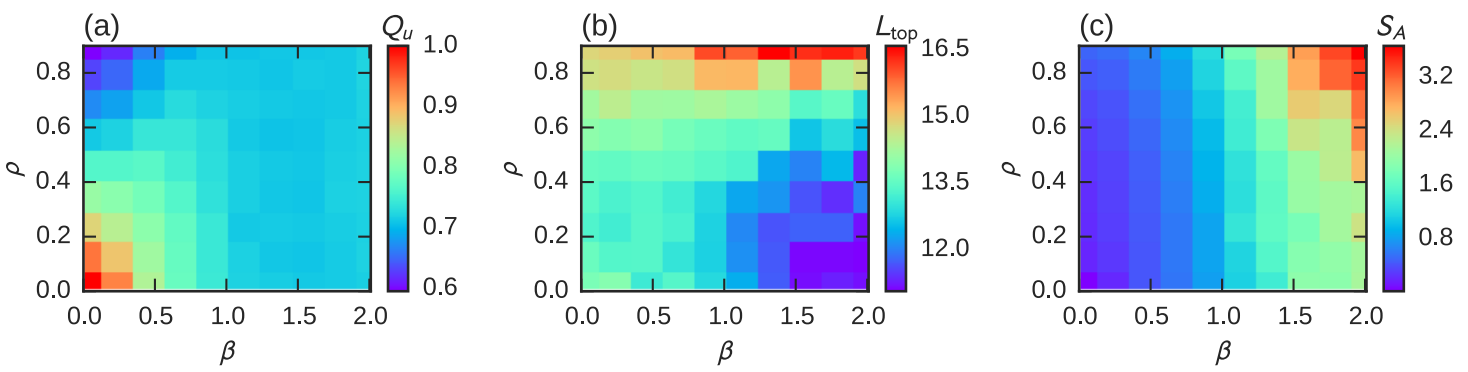

FIGURE 6.3: Phase diagrams of the model for varying $\beta$ and $\rho$ while fixing $\alpha=0.5, f_{n}=0$ and $N=2048$. At zero measurement noise $\left(f_{n}=0\right), \alpha$ has almost no effect on the measured topology. We performed 10 simulations for each combination of parameters and plot the mean results. (a) Unweighted nesting number $Q_{u}$ thresholded at $d \leq 256$. For $\beta<1, Q_{u}$ can be changed by tuning $\rho$. For $\beta>1, Q_{u}$ becomes independent of $\rho$ (at $Q_{u}^{\mathrm{eq}} \approx 0.75$ ). The model values are larger than but relatively close to measured ones. (b) Mean topological length $L_{\text {top }}$. For small $\rho$, topological length can be varied by tuning $\beta$, for large $\rho$, it becomes independent at $L_{\text {top }}^{\text {eq }} \approx 16$. The model values are much larger than measured ones. (c) Normalized areole area standard deviations. For $\beta<1, S_{A}$ is independent of $\rho$.

distinguish two different regimes. For values of $\beta<1$, the nesting number can be varied between approximately 0.6 and 1.0 by tuning $\rho$. The mean topological length is approximately constant at $L_{\text {top }} \approx 14$, and the normalized areole area standard deviation $S_{A}$ is independent of $\rho$. For $\beta>1$, the nesting number becomes independent of both $\rho$ and $\beta$ at a value of approximately 0.75 . The mean topological lengths vary between approximately 11 and 16.5 , and $S_{A}$ varies with $\rho$.

The independence of $Q_{u}$ from either parameter in the regime $\beta>1$ can be explained by the fact that if $p_{\text {split }}$ becomes large enough to introduce new veins at almost every time step, the effects of different loop sizes (controlled by $\rho$ ) become irrelevant.

Figure 6.4 further shows the same three observables in simulated networks with higher measurement noise $\left(f_{n}=0.2\right)$. The qualitative behavior of the 

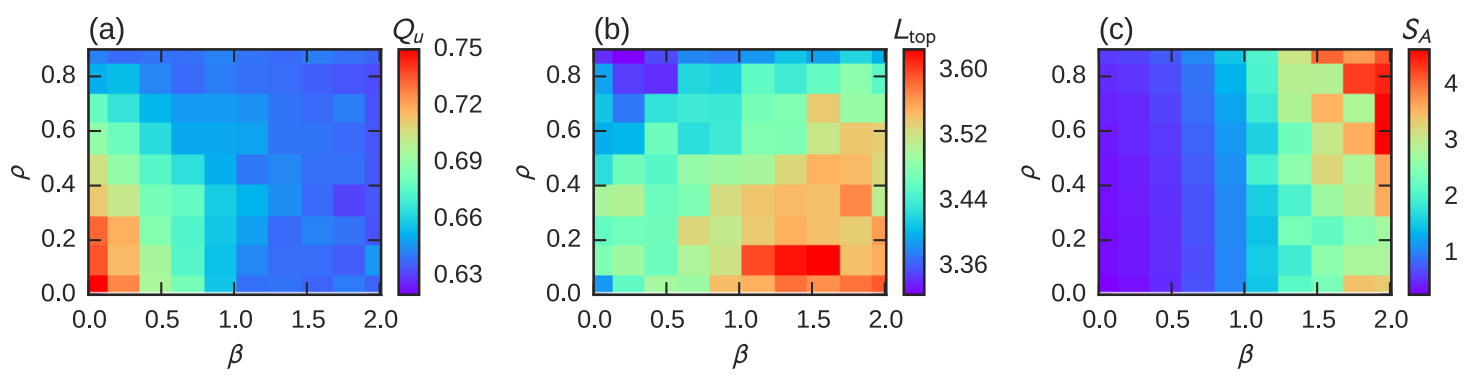

FIGURE 6.4: Phase diagrams of the model for varying $\beta$ and $\rho$ while fixing $\alpha=0.5, f_{n}=0.2$ and $N=2048$. The nesting number $Q_{u}$ behaves qualitatively similarly to the no-noise case Figure 6.3. The values of $L_{\text {top }}$ and $Q_{u}$ are significantly lowered by the presence of measurement noise, $S_{A}$ is not changed much.

nesting number is similar to the no-noise case, but the mean topological lengths are significantly shorter because even small amounts of noise break vein tapering in the model. Similarly, the maximum values of $Q_{u}$ are around 0.75 because hierarchical symmetry is broken by noise (see also Figure 6.2 for the visual effects of measurement noise). Thus, $L_{\text {top }}$ is much less robust against noise as compared to $Q_{u}$.

\subsubsection{Comparison with the data set}
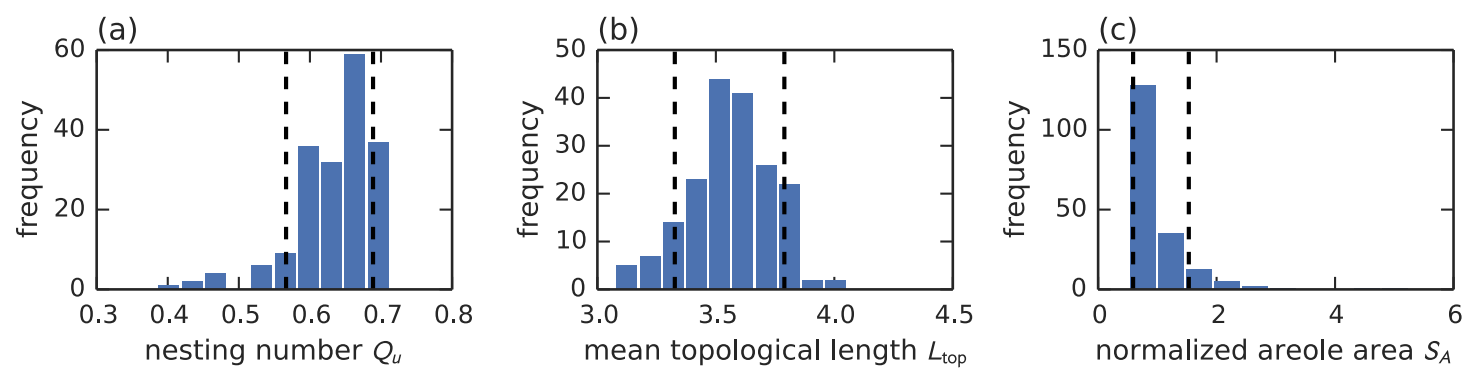

FIGURE 6.5: The same observables as in Figure 6.3 as measured in the data set. Vertical dashed lines are interdecile range, encompassing $80 \%$ of the observed data. (a) The interdecile range is $[0.57,0.69]$. (b) The interdecile range is $[3.33,3.79]$. (c) The interdecile range is $[0.59,1.53]$.

We proceed to compare the model results with our data set. Figure 6.5 shows the observed distributions of the three metrics considered together with their $10 \%$ and $90 \%$ percentiles (the interdecile range). The observed values of $L_{\text {top }}$ and $S_{A}$ are consistent with the model parameter range $f_{n}>0$ and $\beta<1$ and thus constrain the model parameter space. Clearly, measurement noise has a visible effect on the measurements while noise controlling areole size during morphogenesis must be comparatively small. It must be noted that what we term measurement noise in the simulations may be a combination of actual measurement noise and another kind of variability in vein thickness that cannot be disentangled using this model. 
Comparing Figure 6.5 to Figure 6.4, we see that roughly $80 \%$ of the topological variation in the cleared leaf data (as enclosed by the interdecile range) is reproduced by the model (i.e., the model produces the same range of values of the observables as found in the data set).
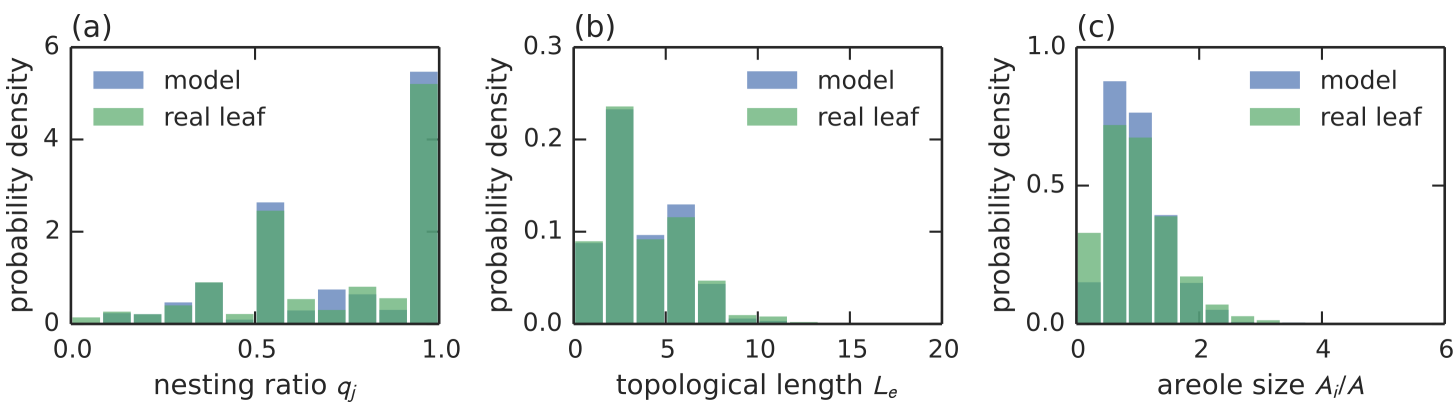

FIGURE 6.6: Comparison of topological length, nesting ratio, and areole area statistics between model and a real leaf with comparatively high nesting number (Protium grandifolium, see Figure 5.4 (a)). The parameters used are $\alpha=0.2, \beta=0.4, \rho=0.25, f_{n}=0.1$; the final network had 1038 loops. This is a low noise setting. Except for the areole sizes where the real leaf contains more small areoles, the distributions fit quite well, in particular the nesting ratios. This is quantified by KS tests. (a) $D_{\mathrm{KS}}=0.03, p=0.33$. (b) $D_{\mathrm{KS}}=0.02$, $p=0.12$. (c) $D_{\mathrm{KS}}=0.08, p<0.001$.

It is possible to reproduce the topological length and nesting ratio statistics of select leaves relatively well by tuning the model parameters. The parameters were tuned by hand until good agreement was reached as measured by KS tests between the distributions of nesting ratios and topological lengths. Very good agreement of the topological statistics can be achieved for highly nested leaves such as Protium grandifolium, the agreement for areole areas is not as good but still acceptable (see Figure 6.6). For less highly nested leaves such as Dalbergia miscolobium, less good agreement of the nesting ratio statistics is achieved (see Figure 6.7). This is due to the appearance of a large number of very small nesting ratios $(q<0.2)$ that may be due to additive nesting structures (see Chapter $\mathrm{C}$ of the Appendix for an explanation of this terminology) in the real leaf that the model does not reproduce. Curiously, the areole areas are much better reproduced in the less highly nested model as compared to the highly nested one.

Because the parameter tuning was done by hand, we do not expect the results to be optimal. Additionally, due to the stochastic nature of the model the same parameters can lead to different statistics of the final network. They are, however, distributed around a characteristic mean value. Still, good agreement of the statistics was achieved, further demonstrating the link between noise and topological features. Low noise parameters lead to highly symmetric, hierarchically nested networks with large topological lengths; high noise parameters lead to more asymmetric, less hierarchically nested networks with shorter topological lengths. 

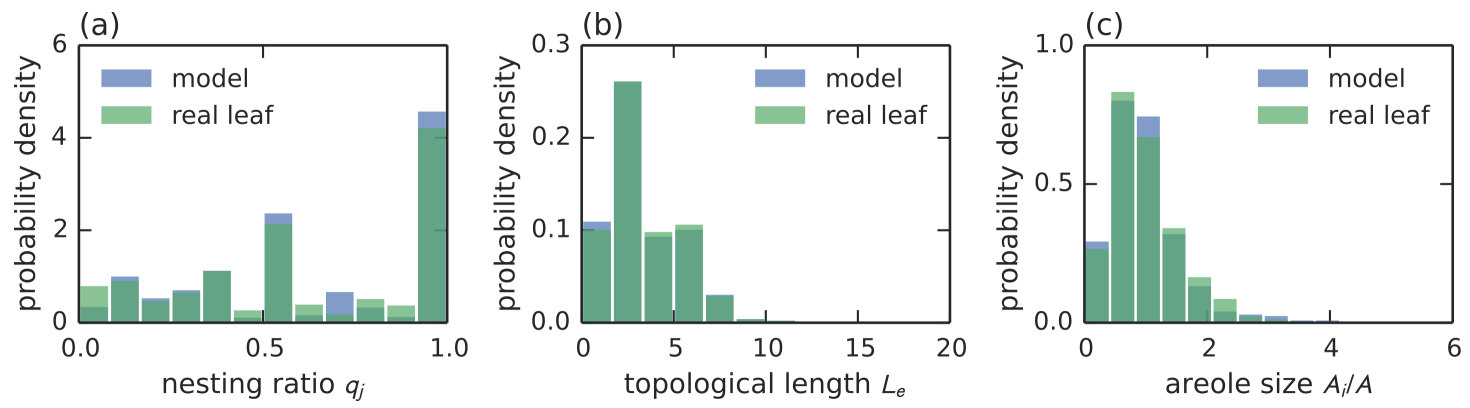

FIGURE 6.7: Comparison of topological length, nesting ratio, and areole area statistics between model and a real leaf with comparatively low nesting number (Dalbergia miscolobium, see Figure 5.4 (b)). The parameters used are $\alpha=0.2, \beta=0.2, \rho=0.75, f_{n}=0.35$; the final network had 896 loops. This is a high noise setting. All distributions fit rather well, the very lowest nesting ratios are not reproduced. (a) $D_{\mathrm{KS}}=0.04, p=0.17$. (b) $D_{\mathrm{KS}}=0.02, p=0.31$. (c) $D_{\mathrm{KS}}=0.03, p=0.40$.

\subsection{Summary and discussion}

We presented an empirical model for the growth and development of reticulate leaf venation patterns. The model is simple, consisting of rectangular loops that grow and divide by the introduction of new veins. This simplicity allows compatibility with the most important biological hypotheses of vein morphogenesis, auxin canalization and mechanical instabilities. The model is stochastic, with tunable noise strength at various stages of development.

We analyzed the model's phase diagram, identifying two phases when varying the growth noise parameter $\beta$. For $\beta>1$, the hierarchical topology of the final networks becomes quasi-independent of the noise parameters, for $\beta<1$ the other parameters can be varied to obtain different hierarchical topologies. Our cleared leaf data set imposes constraints on the possible parameter values of the model to reproduce realistic networks.

We then compared the model networks' mean topological lengths, nesting numbers, and normalized areole area standard deviations to the cleared leaf data set, finding that the bulk of the data (as measured by the interdecile range) can be reproduced by the model. The areole areas served as a geometrical constraint, ensuring that the model networks are realistic in terms of their areole size distributions. We went further and hand-tuned the model parameters to fit the nesting ratio, topological length, and normalized areole area statistics of two test leaves from the data set. We were able to obtain quantitatively good agreement, demonstrating the model's power in reproducing both topological and geometrical features of real leaf networks. We stress that there are some aspects of real leaf venation that are not captured by the model, in particular the existence of very low nesting ratios in some leaves. These may stem from biological processes not modeled.

We conclude that, whichever underlying biological mechanism is true, it is likely to involve growth patterns similar to those of the model. In particular, the influence of random noise appears to be a major component and cannot 
be neglected when striving for a realistic description of vein morphogenesis. It is important to stress the difficulty of separating true measurement noise in the data (from imperfect scanner focus, vibrations during scanning, image post-processing, etc...) from variations in vein thickness growth.

As we have shown in Chapter 5, although noise is a large factor, topology appears to be relatively stable at the level of leaf specimen and even species. This suggests the existence of a characteristic combination of random effects that define a specimen and to a lesser degree a species, partly explaining the earlier observations of Chapter 5. 



\section{Discussion and Outlook}

In this chapter, we summarize the results and conclusions presented in Part II once more and discuss their relevance with respect to biology. Furthermore, we propose future work and at the same time discuss possible sources of systematic errors in the data.

\subsection{Summary and discussion of results}

In Part II, we systematically analyzed the topological and geometrical traits of a set of 186 leaves and leaflets, mainly from the Burseraceae family. For each leaf we calculated a "fingerprint" consisting of five well-studied geometrical and three novel topological traits. The resulting feature vectors constituting the phenotypic space of leaf venation were then analyzed by Principal Component Analysis and Factor Analysis, revealing geometry and topology as complementary dimensions.

We then concentrated on topology in terms of nesting ratio statistics, calculating statistical distances between leaf topologies. The statistical distances were found to weakly reflect a genetic relationship between samples, with leaves belonging to the same species generally exhibiting smaller distances. We showed how in the case of two test leaves, there is high visual similarity between nearest neighbors according to our topological distance. However, hierarchical clustering based on topological statistics as measured by our metrics showed that phylogeny is not, or only to a very limited degree, encoded in leaf network topology.

In order to demonstrate the usefulness of our new topological metrics, we used them to improve accuracy in an identification task. The leaves were digitally cut into equally sized fragments, and the fingerprint data was computed for each fragment individually. We then used Linear Discriminant Analysis to identify which specimen or species within our leaf data set a single fragment belongs to. We showed that employing topological features in addition to geometry leads to a highly significant improvement in identification accuracy of the classifier. Additionally, pairwise comparison was performed, deciding whether two samples belong to the same specimen or species. Here, using topological features also lead to an improvement in identification accuracy.

In order to explain the observed values of the topological leaf traits and get an intuition about how they come to be, we considered a simple empirical growth model that is compatible with prevailing hypotheses of vein morphogenesis and includes stochasticity. The model can explain the bulk of our data set in terms of aggregate features such as mean topological length and nesting number. We 
additionally showed how by fine-tuning the model parameters, we were able to obtain good quantitative agreement between model and real leaf topological and geometrical statistics in the case of two test leaves.

Our results demonstrate that hierarchical topology consists an entirely new dimension in the phenotypic space of leaf venation that is approximately orthogonal to geometry, in the sense of PCA and FA. It contains a large amount of additional information about the venation network as shown by its ability to significantly improve specimen identification from fragments. Such identification tasks may be important especially when dealing with leaf fossils where only small parts are available.

Additionally, we showed how this new phenotype may be explained by random noise affecting vein morphogenesis. To our knowledge, modelers of venation development mostly dismiss the effects of stochasticity. Although they are sometimes incorporated, systematic study is severely lacking. Our work shows that noise may indeed be an absolutely crucial ingredient for a description of vein morphogenesis, and surely warrants significantly more work. This is further supported by our results in Part III, where we are able to produce realistic leaf networks from a model of auxin canalization in conjunction with random fluctuations. We stress that these results are complementary to those of Part II in the sense that here, we model the high level (minor vein) network architecture whereas our auxin model can be thought of as modeling the low level (major vein) structure.

\subsection{Future work}

In this section, we expand more on possible future work that would support and enhance the conclusions made so far. We distinguish several main paths of new work, each improving on some aspect or proposing new projects. The order in which we present these ideas is in no way intended to convey a sense of relative importance.

\subsubsection{Global leaf topology survey}

Despite the fact that our data set contains a large variety of leaves and leaf networks, it is severely limited because most specimens belong to the same family (Burseraceae), and even those that do not were all collected in tropical South America. As such, it provides only limited diversity in terms of habitats and growth environments. We believe that it is important to apply the methods outlined in this thesis to a much broader collection of leaves that constitute a truly diverse, global sample of leaf venation, similar to the work [60] but incorporating topology as well. This would imply also measuring more key traits, such as leaf mass per area and photosynthetic assimilation rates, that were unavailable to us. We would hope and expect to see interesting correlations between topology and ecological and environmental factors. 


\subsubsection{Improving data acquisition techniques}

Data acquisition is an inherent factor contributing to readout noise, rendering it difficult to disentangle systematic errors from true fluctuations in the leaf venation. The protocols for leaf clearing and staining appear mature [151], but vectorization or skeletonization methods are still being actively developed. This is valid for both our approach [152] and others such as [153]. Pre-processing steps requiring the use of filters such as Gaussian Blur can subtly distort vein geometry. Thus, even more accurate vectorization methods are needed. More problems with existing methods have been identified [154, 155]. Additionally, although our scans were captured ad very high resolution (6400 dpi), an even higher resolution with the use of microscopy rather than a flatbed scanner could improve data quality.

\subsubsection{Systematic study of developmental noise}

As we have already mentioned, to our knowledge no systematic study of the effects of random noise during vein morphogenesis exists. Our results suggest that random effects have a significant influence on venation geometry and topology, therefore we believe it important to further investigate other models of the underlying biology (auxin canalization [85] or mechanical instabilities [93]) specifically with regard to stochasticity. We believe that such an analysis might uncover the mechanism that is responsible for morphogenesis, or at least rule out some of the proposed models.

\subsubsection{Applications to other fields}

We strongly believe that many fields can strongly benefit from an analysis similar to the one in this thesis. This includes investigating planar or almost planar weighted networks such as those found in the retina, the mammalian neocortex, urban street patterns, power grids, and river deltas, possibly uncovering unexpected relationships. Additionally, our methods are applicable even when the network possesses only an embedding onto some surface of possibly nonzero genus [156].

\subsubsection{Improved topological metrics}

Even though we believe in the power of our topological metrics, nesting number and topological length, they still only capture a small part of the network topology. Topological lengths are affected by noise and because the hierarchical nesting tree is a binary tree, it underlies very strong constraints. Therefore, it would be interesting to consider new metrics that could possibly make use of the dual graph in a more direct fashion. 

Part III

Network Modeling 



\section{Introduction}
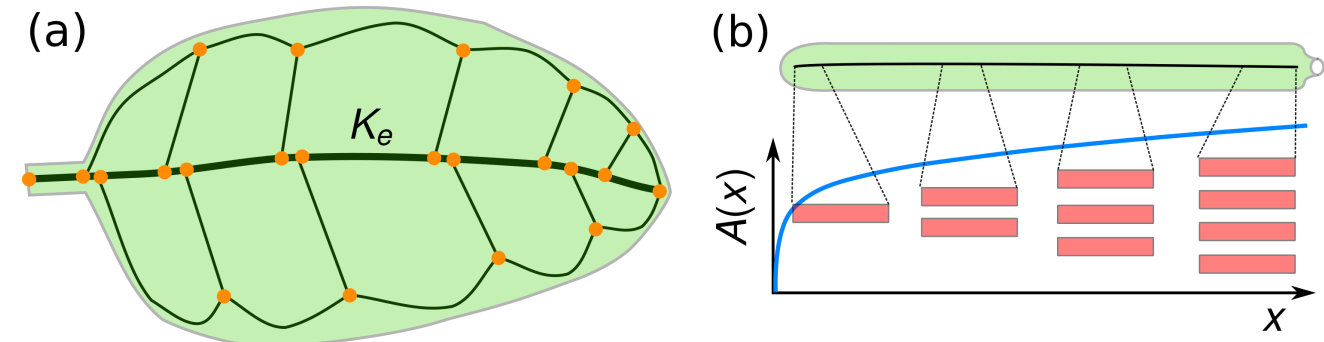

FIGURE 8.1: The levels of coarse-graining appropriate for the analysis of various transportation systems. (a) Two-dimensional, flat leaf venation is modeled as weighted flow network. Each edge $e$ has conductivity $K_{e}$ related to its vein thickness. (b) One-dimensional transport is modeled using a continuum description of total cross-sectional area $A(x)$ of the conducting cells (red rectangles), where $x$ is distance from tip.

In this part of the thesis, we present some results on models of evolution and development of leaf venation. We study the two transport systems xylem [157] and phloem [158]. The xylem, responsible for carrying water into the leaf, is analyzed from the perspectives of development and evolution in two-dimensional, flat angiosperm leaves using a network approach that results in models of overall network topology. The phloem, responsible for transporting photoassimilates (i.e., the results of photosynthesis) out of the leaf to sites of consumption, is analyzed from the perspective of evolution in one-dimensional gymnosperm needles using a microscopic geometric approach that results in a prediction for the scaling behavior of the total conduit cross sectional area as a function of distance from needle tip. Being effectively one-dimensional, conifer needles benefit from a microscopic description, whereas the relevant features of twodimensional dicot leaves are captured more effectively by a network model. Thus, we have two descriptions of different aspects of venation at different levels of coarse-graining. We find that fundamentally, an equivalent mathematical description applies to both evolution and development at both these levels. This may be a coincidence or a telltale sign that nature indeed selects not for the complicated topology or geometry of the venation patterns themselves, but instead for a simple, universal mechanism that is able to produce them in a self-organized fashion.

For modeling evolution, we take a fitness function approach [159], assuming that successful evolutionary designs maximize fitness (i.e., the expected number of offspring). However, because we are interested in very specialized plant organs such as leaves and needles, it is necessary to come up with a proxy for fitness that captures some feature of the organ's design that is likely to 
contribute to overall fitness. In our case it turns out that the proxy function that reproduces overall leaf venation topology is the mean hydrostatic pressure drop in the xylem elements (this was shown in earlier work [71, 72]), whereas the proxy reproducing phloem geometry is dissipated power (shown by the author and others in [45]). In a certain limit, we show that they are equivalent. Fundamentally, our evolutionary models are steady state models that are meant to capture the "average leaf topology" or "average needle geometry" that natural selection may care about.

A theory of linear flow and perturbations on networks is necessary for the network modeling section. Interesting as it is in its own right, we relegate most of the material that is not directly necessary to Chapter D of the appendix. Some of it has interesting applications in the computational physics of power grids (shown by the author and others in [160]).

For modeling development, we focus on the most well-supported hypothesis, called auxin canalization theory [84, 85]. Vein development is assumed to be controlled by the flow of the phytohormone auxin which is produced in the growing leaf embryo, at first diffusing freely and being removed at the petiole. Auxin is then part of a positive feedback mechanism where areas of high flow of an initially homogeneous cellular matrix are modified into vascular precursor cells, further increasing auxin flow. The model captures the essential venation growth dynamics and drives toward a steady state that represents the mature network topology. We find that the crucial ingredient leading to realistic networks similar to those obtained from optimization is the introduction of spatially correlated fluctuations of auxin sources and sinks. This ties in with and supports the results from Part II, where we identified random noise during development as an important factor for producing realistic network topologies.

The rest of this part is organized as follows. First, we introduce evolutionary models of venation networks that are based on the idea of optimizing fitness functions or fitness function proxies. We then apply these principles to the problem of finding the optimal phloem geometry in pine needles. We proceed to consider optimal xylem networks in two-dimensional leaves, extending earlier work [72] to much higher resolution using a clever choice of boundary conditions. Finally, we present a model of auxin canalization that shows how correlated fluctuations in auxin sources and sinks can produce networks very similar to those obtained from optimization procedures and how by tuning the correlation range we can access a continuous family of network topologies.

\subsection{Fitness function models of evolution}

The basic philosophy behind the models of evolution and adaptation we use was put forth very early by Wright [159]. He identified fitness, measured by the expected number of offspring, as the major objective function that is maximized by evolution through natural selection. In a most simplistic view, because populations in the absence of resource limitations or competition grow exponentially 
with growth rate proportional to fitness, the population that is, on average, fitter will in time always overtake the less fit competitors, thus selecting for traits that maximize fitness.

Essentially, the fitness function $f: P \rightarrow \mathbb{R}$ as proposed by Wright maps from the space $P$ of phenotypes to the positive real line. Here, $P$ depends on the model in question and can be continuous or discrete. ${ }^{1}$ Wright envisaged a continuous landscape of hills and valleys, with populations occupying the hills, representing large fitness. In order to increase their fitness, they would have to "move" through valleys or along ridges to the next higher hill. This intuition from picturing $P$ as "basically" $\mathbb{R}^{2}$ is wrong, or at least grossly misleading [161, 162]. Real phenotypic spaces are high-dimensional, possibly containing tens of thousands of phenotypic traits [162]. Such spaces behave quite counter-intuitively, containing various "short-cuts" that allow one to move quickly between high-fitness loci without crossing valleys. Thus, they are often better represented as networks of such connected high-fitness loci [161].

In our case, we need to take a step back and realize that one plant organ such as the leaf can not determine fitness alone, but it can make a contribution to the fitness of the whole organism. We quantify this contribution by considering a proxy function $\pi: P_{\text {leaf }} \rightarrow \mathbb{R}$, where $P_{\text {leaf }} \subset P$ is the phenotypic space of the leaf, which may be maximized or minimized in order to maximize fitness.

A fundamental question then becomes, what is the "right" choice of $\pi$ ? This question is very hard to answer definitively (and possibly, a definitive answer does not even exist!), and one must feel very content indeed when one has identified a candidate whose optimization correctly predicts the observed phenotypes and is consistent with the known biology. In our case, studying leaves and needles, there are a few clues (such as the constructal law, discussed in Section 2.1.9) that can guide one towards towards the right proxy function, but surprises may still be in store. Still, once a good candidate proxy has been identified, it can actually be more informative about biology than the very abstract fitness function itself. Finding and interpreting the proxy functions is the main goal of the following chapters dealing with evolutionary modeling.

${ }^{1}$ Indirectly, the phenotype is in some way determined also by the genotype, but this distinction further complicates the argument and is not relevant to our analysis. 



\section{Optimal Geometry of Pine Needle Phloem}

\subsection{Introduction}

Being effectively one-dimensional, conifer needles provide a particularly simple model system for transport phenomena in plants, because there are no complications arising from vein branching. Most conifer needles possess only one or two parallel veins close to the center of the needle. Despite their simplicity, transport of water through the xylem and nutrients through the phloem are not well understood.

In this chapter, we concentrate on an analytical description of photoassimilate transport in the phloem of conifer needles, explaining measured phloem bundle geometry using a power dissipation minimization argument. Our results complement earlier work on the scaling of single phloem cells on the scale of a whole tree [30, 39, 42]. Furthermore, we augment the simple analytical model by numerical simulations, relaxing simplifying assumptions. Finally, we show that, contrary to expectations, overall needle phloem geometry cannot be explained by flow rate optimization.

The results discussed in this chapter were published in part in [45].

\subsection{Mathematical model of sugar flow in needle phloem}

In this section, we describe a simple, analytically solvable model of sugar translocation, based on earlier work $[163,164]$. We consider a one-dimensional conifer needle of length $L$ containing a vascular bundle of phloem sieve elements with equal cross-sectional area $A_{0}$. This approximation can be justified by data [160]. The number of sieve elements (conducting channels) at position $x$ from the tip of the needle is $N(x)$, which is typically very small at the tip $(x=0)$ and steadily rises towards the petiole $(x=L)$ (see Figure 9.1). This continuum description is unaffected by the exact mechanism of adding new conducting channels (branching of existing tubes or appearance of additional, new tubes).

\subsubsection{Transport equations}

We define the volume flow rate of sap $Q(x)\left[\mathrm{m}^{3} \mathrm{~s}^{-1}\right]$ and solute concentration $c(x)\left[\mathrm{mol} \mathrm{m}^{-3}\right]$, as well as total sugar current $J(x)=Q(x) c(x)\left[\mathrm{mol} \mathrm{s}^{-1}\right]$. Because 


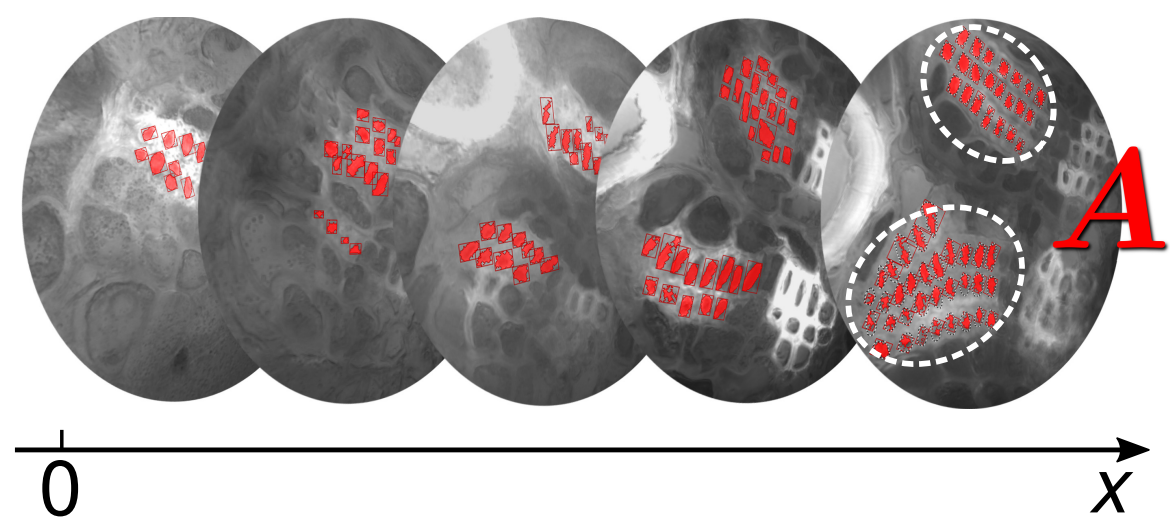

FIGURE 9.1: Phloem geometry in a pine needle. Micrograph cross sections of the phloem of an Abies nordmanniana needle taken at distances $x=0.4,0.8,1.6,2.9,4.9 \mathrm{~mm}$ from the tip. The diameter of the circular cross sections is $100 \mu \mathrm{m}$. The conductive phloem area $A(x)$ and the number of cells (red) increases with distance $x$ from the needle tip while the size of individual cells remains roughly constant.

phloem loading in conifers is thought to be passive (i.e., relying on cell-tocell diffusion) [34], the rate $\Gamma(x)$ of sugar loading per length depends only on mesophyll photosynthetic rate and needle circumference. Since the conifer needle circumference is constant to a very good approximation (except very close to the tip) and the photosynthetic rate should not depend on position, we assume that $\Gamma(x) \equiv \Gamma$ is a constant. The continuity equation for sugar can therefore be expressed as

$$
\frac{d J}{d x}=\Gamma
$$

Similarly, we can express the continuity equation for water in the form

$$
\frac{d Q}{d x}=2 \frac{L_{p} A(x)}{r_{0}}(f(\Delta c)-\Delta p) .
$$

Here, $L_{p}$ is the permeability of the sieve element membranes, $A(x)=N(x) A_{0}$ is the total cross sectional area of all sieve tubes, $r_{0}$ is the radius of a single tube, $\Delta p$ is the hydrostatic pressure difference between phloem cells and surrounding tissue, $\Delta c$ is the sugar concentration difference between phloem cells and surrounding tissue, and $f(\Delta c)$ is a law relating concentration gradient to osmotic pressure. An approximate choice for $f$ is the linear van 't Hoff law,

$$
f(\Delta c)=R T \Delta c
$$

where $R$ is the universal gas constant and $T$ is temperature. In practice, the error introduced by the van 't Hoff law for sugar concentrations in phloem sap is on the order of $10 \%$ ([165], $c<1 \mathrm{M})$.

We use Darcy's law to describe flow velocity in terms of hydrostatic pressure 
gradient,

$$
u(x)=-\frac{k}{\eta} \frac{d p}{d x}
$$

where $u(x)=Q(x) / A(x)$ is bulk flow velocity, $k$ is a geometrical constant (for cylindrical tubes, $\left.k=A_{0} / 8 \pi\right), \eta$ is the sap viscosity and $p$ is the hydrostatic pressure. Phloem sap viscosity is typically about 5 times that of water [39], and Darcy's law holds because flow in the phloem is laminar to a very good approximation.

Imposing the boundary condition $J(0)=0$ (no flow at the tip), we can integrate equation (9.1) to obtain $J(x)=\Gamma x$. The total sugar flow leaving the needle is therefore $J(L)=\Gamma L$. Using the van 't Hoff law and assuming that both the hydrostatic pressure and the sugar concentration in the tissue surrounding the vascular bundle are constant and uniform, we end up with the following set of differential equations approximately describing sugar loading and transport in a conifer needle:

$$
\begin{aligned}
Q(x) & =-\frac{k}{\eta} A(x) \frac{d p}{d x} \\
\frac{d Q}{d x} & =2 \frac{L_{p} A(x)}{r_{0}}\left(R T \frac{\Gamma x}{Q(x)}-p(x)+\Delta\right),
\end{aligned}
$$

where $\Delta=R T \tilde{c}-\tilde{p}$ with constant external solute concentration $\tilde{c}$ and constant external hydrostatic pressure $\tilde{p}$. Choosing the needle length $L$ as the length scale, it is possible to nondimensionalize this system, obtaining

$$
\begin{aligned}
& Q(x)=-A(x) \frac{d p}{d x} \\
& \zeta \frac{d Q}{d x}=2 A(x)\left(\frac{x}{Q(x)}-p(x)+\tilde{\Delta}\right),
\end{aligned}
$$

where we introduced the dimensionless control parameters $\zeta=k r_{0} /\left(\eta L^{2} L_{p}\right)$, the ratio between typical phloem bulk and membrane conductivity, and $\tilde{\Delta}=$ $(k /(R T \Gamma \eta))^{1 / 2} \Delta$, the external pressure normalized by the geometric mean of typical phloem bulk and lateral pressure drop. Using the cylinder approximation $k=\pi r_{0}^{2} / 8$ as well as the numerical values for viscosity $\eta \approx 5 \times 10^{-3}$ Pas, sieve element radius $r_{0} \approx 1 \times 10^{-5} \mathrm{~m}$, needle length $L \approx 0.1 \mathrm{~m}$, and membrane permeability $L_{p} \approx 5 \times 10^{-14} \mathrm{~m} \mathrm{~Pa}^{-1} \mathrm{~s}^{-1}$ [39], we estimate a value of $\zeta \approx 10^{4}$ in realistic conditions. Estimating $\tilde{\Delta}$ is more difficult because the value of $\Gamma$ is unknown. We take $\tilde{\Delta}=-10$ to emphasize that in the absence of sugar in the phloem, water must leave the conductive tissue. The precise numerical value of 
$\tilde{\Delta}$ is not critical for the qualitative results. ${ }^{1}$

We solved the system (9.6) numerically for the two relevant geometries $A(x)=$ 1 and $A(x)=x^{1 / 2}$ with the additional boundary condition $p(0)=0$. The second geometry was chosen because it will arise later as the optimum phloem geometry. In both cases, the concentration profile $c(x)$ is reasonably close to constant far from the tip (see Figure 9.2), such that in the following, we will make the additional approximation $c(x) \equiv c_{0}$. Similar results can also be found in [30, 164].
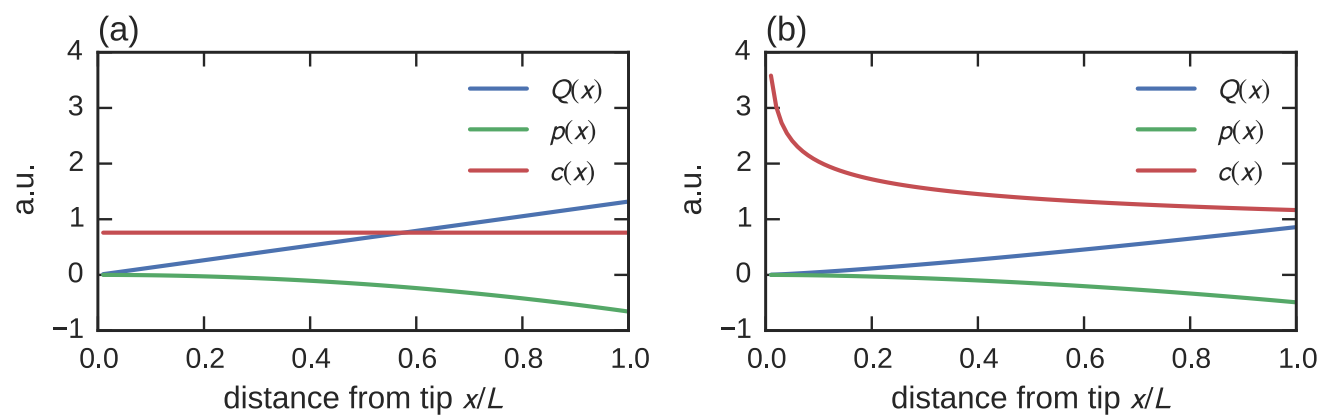

FIGURE 9.2: Numerical solution of the system of ordinary differential equations (9.6). We chose the values $\zeta=10^{4}, \tilde{\Delta}=-10$. The $y$ axis has arbitrary units, showing only qualitative behavior. (a) Solution with constant geometry, $A(x)=1$. The concentration profile is almost perfectly constant. (b) Solution with realistic geometry, $A(x)=x^{1 / 2}$ (see also Section 9.3). The concentration profile is reasonably close to constant for $x>0.2$.

\subsubsection{Optimizing the energetic cost of transport}

There are two factors contributing to the energetic cost of transport through the phloem. First, phloem cells themselves are alive and thus require energy to be sustained. This is an energetic "maintenance cost", which we assume to be proportional to total conductive volume $V_{0}=\int_{0}^{L} A(x) d x$. Second, there is viscous power dissipation due to internal friction in the fluid during transport. High dissipation forces the plant to maintain stronger osmotic gradients between source and sink tissue in order to support a desired flow rate, which translates to higher energetic cost of transport. Viscous power dissipation in a conduit of infinitesimal length is $d P=-(Q d p+p d Q)$. With the approximation $c(x) \equiv c_{0}$, we easily derive $d Q / d x \equiv$ const from equation (9.1). This knowledge allows us to find the total viscous power dissipation along the length of the needle by

\footnotetext{
${ }^{1}$ Additional numerical experiments showed that the solutions are practically independent of $\tilde{\Delta}$ for a wide range of values of either sign. Indeed, it can be shown that the set of equations (9.6) is equivalent to a single second-order differential equation that is independent of $\tilde{\Delta}$. However, this system is numerically more difficult to solve.
} 
integration as

$$
P=Q(L) \Delta p=Q(L) \int_{0}^{L} \frac{d p}{d x} d x=\frac{\eta}{k} \frac{\Gamma^{2}}{c_{0}^{2}} \int_{0}^{L} \frac{x}{A(x)} d x,
$$

where we fixed $p(0)=0$. Using the method of Lagrange multipliers to find the optimum phloem geometry $A(x)$ minimizing power dissipation $P$ under the volume preserving constraint $\int_{0}^{L} A(x) d x \equiv$ const, we obtain the optimization functional

$$
\Pi=\int_{0}^{L} \frac{x}{A(x)} d x+\lambda\left(\int_{0}^{L} A(x) d x-V_{0}\right)
$$

The volume constraint models the assumption that the plant has a fixed "energy budget" to maintain its phloem cells. Equivalently, one can think of a fixed amount of cell material that is available to build the phloem. The functional (9.8) is minimized by

$$
A(x)=\frac{3}{2} \frac{V_{0}}{L}\left(\frac{x}{L}\right)^{1 / 2} .
$$

This is a scaling law with $A(x) \sim x^{1 / 2}$. We note that this law is the same the one found by Zwieniecki et al. who applied similar reasoning to the case of water transport in the xylem of pine needles [71], deriving an optimization principle for the tapering of the xylem bundle. Remarkably, the mathematical form of their optimization functional is identical to ours, even though their ansatz is quite different. This convergence of models points towards an interesting mathematical connection between models of transport of solutes and transport of water in plants. In a sense, it is a lucky coincidence for the plant that the identical vascular geometry optimizes both xylem and phloem transport.

\subsubsection{Generalized constraints}

We note that the constraint $\int_{0}^{L} A(x) d x=V_{0}$ can be modified by allowing a more general dependence on phloem geometry through a parameter by fixing

$$
K=\int_{0}^{L} A(x)^{\gamma} d x .
$$

Minimizing equation (9.7) under this constraint leads to the modified scaling law $A(x) \sim x^{1 /(1+\gamma)}$, which is always sub-linear. However, with this ansatz we lose the intuitive geometrical interpretation of the constraint as the total conductive volume $V_{0}$. 


\subsection{Experimental results}

The scaling behavior of phloem geometry was investigated experimentally by the group of Johannes Liesche and Alexander Schulz at the University of Copenhagen in order to compare to our theoretical results. To this end, needles from four conifer species were collected and measurements taken. The species were selected from three genera of conifers for their high diversity in needle size and habitat (see Figure 9.3). The conifers chosen were Picea omorika, which natu-

(a)

Picea omorika

Pinus palustris (b)

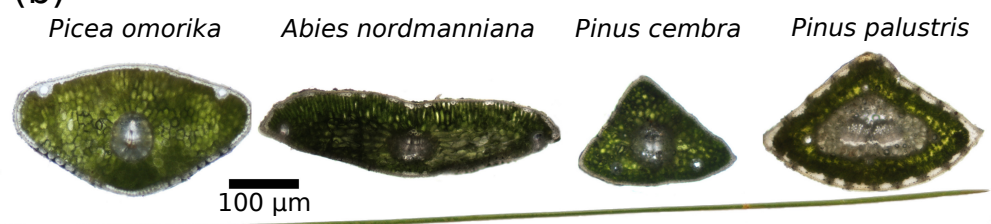

$10 \mathrm{~cm}$

FIGURE 9.3: Conifer needle characteristics. (a) Photographs of representative needle specimens of the species studied. Lengths range between $1 \mathrm{~cm}$ (P. omorika) and $30 \mathrm{~cm}$ (P. palustris). (b) Micrographs showing cross sections of typical needle specimens. The vascular tissue in the center of each needle (light color) is clearly discernible.

rally occurs only in a small region in Serbia (typical needle length $1 \mathrm{~cm}$ ), Abies nordmanniana, which naturally occurs in a region from the Western Caucasus to North-East Turkey (typical needle length $2 \mathrm{~cm}$ ), Pinus cembra, which naturally occurs in the Alps and Carpathians (typical needle length $10 \mathrm{~cm}$ ), and Pinus palustris, which naturally occurs in the South-Eastern United States (typical needle length of a juvenile plant $25 \mathrm{~cm}$ ).

From each species, 3-6 needles were collected, sectioned, live phloem cells chemically stained and the sections imaged. Image analysis was used to extract the total conductive area $A(x)$ for each needle. Figure 9.4 shows both the measured total sieve element areas $A(x)$ as well as the normalized sieve element areas $A(x) / A(L)$ on a logarithmic scale. Clearly, the needle data falls closely within the range predicted by the optimization arguments presented in the previous section.

Not much other work exists investigating phloem geometry in linear leaves such as needles. Evert $e t$ al. measured phloem conductive area in several grass species (maize [166], barley [167], and sugarcane [168]). Out of these, the results for barley are in good agreement with our scaling law, whereas maize and sugarcane only show approximately sub-linear scaling. Since grass leaves are not perfectly one-dimensional, consisting of several parallel, interconnected veins, stronger deviations from our scaling law for ideal one-dimensional leaves are to be expected. However, scaling is still sub-linear and could be explained by a modified constraint such as (9.10). 

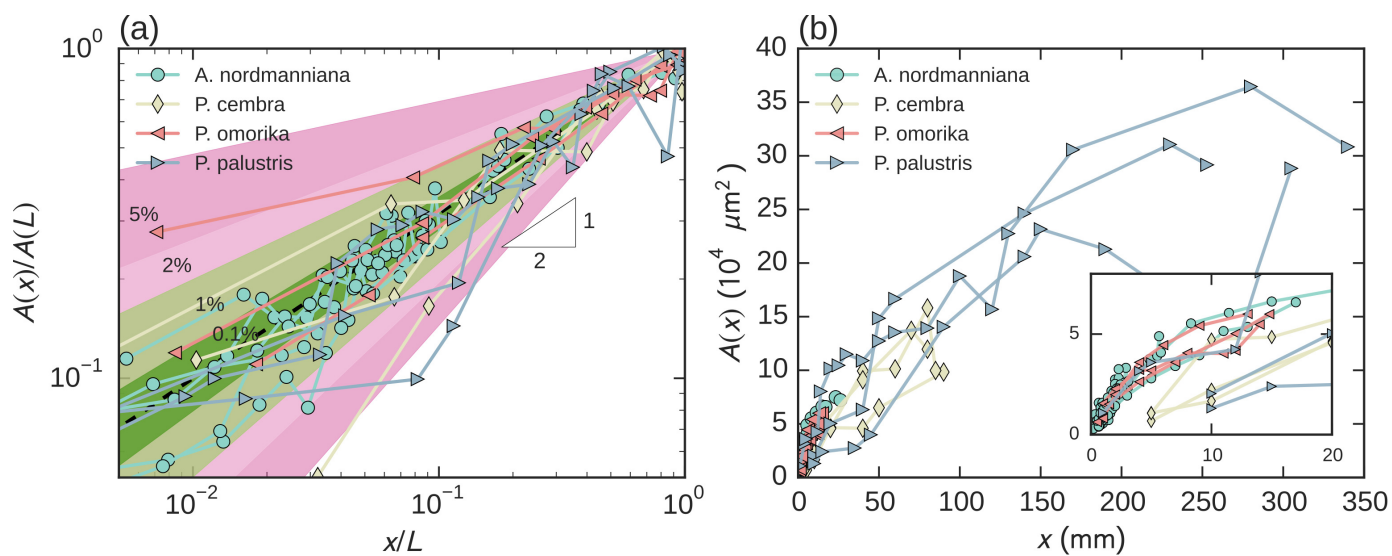

FIGURE 9.4: Comparison of experimental sieve element area data with prediction from optimization. Lines connect data from the same needle. (a) Double-logarithmic plot of normalized total sieve element area $A(x) / A(L)$ against normalized distance from tip $x / L$. The dashed line corresponds to $y=(x / L)^{1 / 2}$. The colored regions correspond to $y_{\alpha}=(x / L)^{\alpha}$ whose power dissipations exceed the optimum at $\alpha=0.5$ by the given percentages. Most of the needles fall within the $1 \%$ range $\alpha \in[0.35,0.65]$. (b) Total conductive phloem area as function of distance from tip for all analyzed needles. The inset shows details near $x=0$ for the shorter species.

\subsection{Alternative objective functions}

Although successful, the model described in Section 9.2 suffers from the same problem as virtually all optimization based explanations of biological form and function. It is in practice nearly impossible to verify that the objective function employed is really the one nature herself had chosen as well. In this section, we investigate a number of alternative but still reasonable optimization models and show that they do not lead to the same prediction for vein scaling as the model (9.8).

The most important alternative model was proposed in [30,39] by deriving a model for long range translocation of solutes in the phloem of whole trees. The authors modeled a whole tree as a system of hydraulic resistors, taking into account contributions from source (leaf), transport (branches and trunk) and sink (fruits, roots) tissue, $R=R_{\text {source }}+R_{\text {trans }}+R_{\text {sink, }}$, and showed that observed scaling behavior of single phloem vessel radius could be explained using a principle of sugar flow rate maximization. This is valid on the level of the whole tree (in contrast to our model, which is valid on the level of a single needle, but takes into account flow through the whole vascular bundle and not just single vessels). Thus, sugar flow rate $j$ or volume flow $J$ are identified as viable candidates for optimization models. In the following, we analyze a single needle model under the assumption of flow rate optimization, keeping the constraint $\int_{0}^{L} A(x) d x=V_{0}$. We show that flow rate optimization can not be used to explain the observed needle geometry. 


\subsubsection{Flow rate and volume flow do not explain needle phloem}

A straightforward, obvious goal for the needle is to maximize flow rate $j(L)$ or volume flow rate $J(L)$ of sugars at the petiole. These are defined as

$$
\begin{aligned}
& J(L)=Q(L) c(L) \\
& j(L)=\frac{Q(L) c(L)}{A(L)} .
\end{aligned}
$$

We note that under the simplifying first order assumptions from Section 9.2, $J(L)=\Gamma L$, which is a constant and does not depend on geometry. Thus, maximizing (9.11) does not explain phloem geometry.

To show that maximizing flow rate (9.12) can not explain phloem geometry either, we note that the family of ansatz functions

$$
A_{\gamma}(x)=\frac{\gamma+1}{L}\left(\frac{x}{L}\right)^{\gamma}
$$

all satisfy the volume fixing constraint. Since $j(L)=J(L) / A(L)=\Gamma L / A(L)$, we can insert our ansatz function and see that flow rate at the petiole $j(L)$ diverges as $\gamma \rightarrow \infty$. Therefore, flow rate maximization at the petiole is not responsible for the observed phloem geometry, either.

Finally, we can consider the average flow rate within the needle,

$$
\langle j\rangle=\frac{1}{L} \int_{0}^{L} j(x) d x=\frac{1}{L} \int_{0}^{L} \frac{J(x)}{A(x)} d x .
$$

Under the assumptions from Section 9.2, we have $J(x)=\Gamma x$, such that $\langle j\rangle$ is formally identical to the functional describing power dissipation in the needle. Therefore, it attains its critical point at $A(x) \sim x^{1 / 2}$. However, it is easy to see by comparing

$$
\frac{\langle j\rangle_{A(x) \sim x^{1 / 2}}}{\langle j\rangle_{A(x) \sim 1}}=\frac{8}{9},
$$

that this critical point in fact represents a minimum of the average sugar flow rate. This result is interesting in its own right. A conifer needle appears to minimize power dissipation due to flow by adjusting its geometry while at the same time minimizing average flow rate.

\subsection{Summary and further work}

We have studied a simple model of nutrient transport in the phloem of pine needles and compared its predictions to direct measurements of phloem geometry in four conifer species. The model is based on the optimization of viscous 
power dissipation during flow. This is biologically sensible because, in contrast to the passive transport of water in the xylem, the plant needs to spend energy in order to keep up an osmotic gradient along the entire length of the transport pathway. Therefore, in this context, minimizing energy expenditure is useful, and potentially leading to a selective advantage.

Analytically optimizing power dissipation in the needle under the constraint of constant total volume leads to the prediction that the total cross-sectional area of all phloem cells should scale as $A(x) \sim x^{1 / 2}$ as a function of distance from the needle tip. This scaling is roughly observed in the experimental data.

We then investigated alternatives, in particular flow rate optimization, which had been observed on the whole-tree-vessel-element scale before. We showed that on the needle-vessel-bundle scale, it cannot hold true.

Our results are not in contradiction to flow rate optimization on the singlevessel scale. Rather, they complement each other, showing how nature has once again chosen a structure for plant organs and tissues that is optimal in multiple ways.

Simple models such as the one from this chapter can serve as a stepping stone for more elaborate ones, providing a more refined description of transport in biological organisms. This includes the network models studied in the next chapter. Because of the mathematical equivalence between the power dissipation and pressure drop objective functions, similar results for xylem and phloem network are expected (and indeed, as noted earlier, xylem and phloem occur together in the vascular bundle).

It is highly interesting that recently, similar scaling relations were found in kelp (a type of brown algae) [169]. Kelp and vascular plants are approximately equally distantly related to animals. Thus, this is an excellent example of convergent evolution (note though that for biological reasons the observed scaling power in kelp is $1 / 3$ instead of $1 / 2$ in conifers).

It may be worthwhile to further study models with different loading functions in order to elucidate the effect of loading on optimal phloem geometry. So far, we only analyzed uniform loading $d J / d x=\Gamma$. However, depending on phloem loading strategy, plants may try to achieve a constant photoassimilate concentration in the phloem more actively. For instance, in [43], "target loading" with $d J / d x=a(c(x)-\sigma)$ with target concentration $\sigma$ and loading rate $a$ was proposed. 



\section{Robust Optimal Transport Networks and Auxin Canalization}

In this chapter, we analyze robust optimal transport networks and dynamical models of venation development in growing plants. Previous work [72, 114] has shown how optimal transport networks that are robust against damage or fluctuations develop a reticulate, nested structure as an evolutionary adaptation. We extend this work in several directions. First, we investigate the question of possible sequences in which loops can develop over the course of evolution. This is done by considering two models of damage strength, assuming that different levels of hierarchical reticulation guard against different strengths of damage (e.g., it is not necessary for the leaf to form large anastomoses if only small damage is expected). Comparison with the fossil record then suggests that reticulate venation may have first developed to guard against embolisms but not against pathogens.

Then, we develop a simple, heuristic method to analyze substructures of the leaf network that had previously been unavailable due to computational limitations. We do this by a clever choice of boundary conditions, effectively modeling parts of one single leaf structure. This allows for "zooming in" to small sections and analyzing the optimal hierarchical topology. We compare our results to real leaves and find good visual agreement.

Finally, we connect our optimal robust networks to development. We show that the simplest possible model of auxin canalization is able to replicate the optimal topology if one allows for spatially correlated fluctuations in auxin sources and sinks. Further, we demonstrate that by tuning the correlation range of spatial fluctuations, it is possible to access a continuous family of reticulate network topologies.

\subsection{Robust optimal transport networks}

In this section we review the theory of optimal robust transport networks. Robustness is understood to refer to resilience against expected damage in the network, as well as against fluctuations in the load. Even though the model is designed to apply to biological networks in leaves, it is mathematically equivalent to models used in the theory of power grids. Thus, the results may be just as applicable there (the theory of damage in power grids is reviewed for instance in $[170,171])$. Mathematicians also extensively study such networks; many results can be found in [172]. 


\subsubsection{Modeling leaf xylem networks}

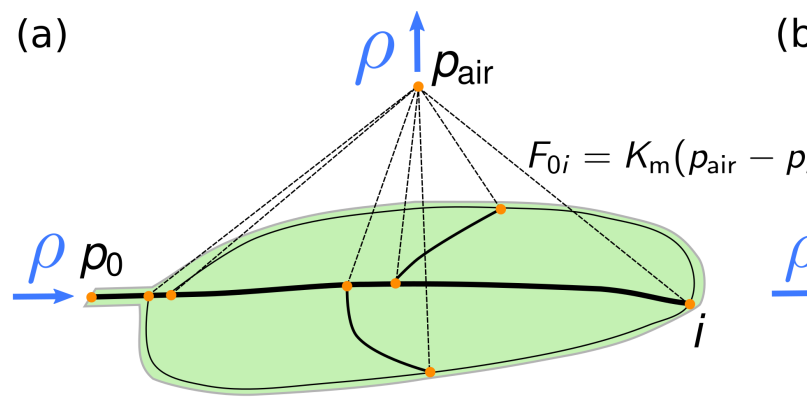

(b)

FIGURE 10.1: Simplifying leaf xylem network models. (a) A network model including the outside-xylem pathways through the mesophyll. The total evaporating water flow $\rho$ enters the network at the petiole and leaves it at the node representing the surrounding air. The flow through each small section of mesophyll surrounding node $i$ is $F_{0 i}=K_{\mathrm{m}}\left(p_{\text {air }}-p_{i}\right)$. (b) Simplified model taking into account the relative hydraulic resistance between xylem and outside-xylem pathways. In the limit $K_{\mathrm{m}} \rightarrow 0$, evaporation is uniform and $\rho_{i}=-\rho / N$ becomes a boundary condition.

We adopt the following simplified model for leaf xylem networks. Venation is modeled as a graph containing $N+2$ nodes. The first $N+1$ nodes represent the leaf venation network itself, with node 0 corresponding to the petiole, where the leaf is attached to the rest of the plant. Thus, the subgraph consisting of these nodes is planar and models water flow through the hierarchical leaf venation network. The nodes $1, \ldots, N$ are assumed to be roughly uniformly distributed on the leaf lamina. Each of them is connected to the outside node $N+1$, representing the ambient air into which water is assumed to evaporate (see Figure 10.1 (a)). Water flow follows the law

$$
F_{i j}=K_{i j}\left(p_{j}-p_{i}\right),
$$

where $K_{i j}=K_{j i}$ is the hydraulic conductivity between nodes $i$ and $j$, and $p_{i}$ is the hydrostatic pressure at node $i$. The water vapor pressure in the ambient air is $p_{N+1} \equiv p_{\text {air. }}$. At each node $i$, the continuity equation

$$
\sum_{j} F_{i j}+\rho_{i}=0
$$

holds, where the sum runs over all neighbors $j$ of $i$. Here, $\rho_{i}$ is the net flow at node $i$. The hydraulic conductivity inside the venation network is related to vein thickness, but because the precise geometry inside the vein is complicated, Poiseuille's law does not hold directly (see the introduction as well as [173]). Still, flow is pressure-driven. For the outside-xylem pathway we assume that all stomata are opened equally and that the hydraulic resistance water encounters on its way out is independent of position on the leaf blade. Thus we have for the 
flows through the mesophyll and stomata

$$
F_{0 i}=K_{\mathrm{m}}\left(p_{\text {air }}-p_{i}\right),
$$

where $K_{\mathrm{m}}$ is the mesophyll-pathway hydraulic conductivity. In addition, the steady-state relation $\rho=\sum_{i} F_{0 i}$ holds, where $\rho$ is the total flow of water entering the leaf network at the petiole. The model is visualized in Figure 10.1 (a).

\subsubsection{Decoupling the hydraulic pathways}

We now derive the decomposition (2.9) from the introduction. The derivation allows us to further simplify the model by neglecting the outside-xylem pathways and provides an intuition for the way water moves through a leaf. We consider the whole leaf as a single hydraulic resistor with conductivity $K_{\text {leaf }}$ and write

$$
\begin{aligned}
\rho & =K_{\text {leaf }}\left(p_{\text {air }}-p_{0}\right) \\
& =K_{\text {leaf }}(\underbrace{p_{\text {air }}-p_{i}}_{=F_{0 i} / K_{\mathrm{m}}}-\left(p_{i}-p_{0}\right)) .
\end{aligned}
$$

Summing the last equation over the internal network nodes $i \in\{1, \ldots, N\}$ and rearranging, we are left with

$$
\begin{aligned}
\frac{1}{K_{\text {leaf }}} & =\frac{1}{N K_{\mathrm{m}}}+\frac{1}{\rho} \frac{1}{N} \sum_{i=1}^{N}\left(p_{i}-p_{0}\right) \\
& =\frac{1}{K_{\mathrm{ox}}}+\frac{1}{K_{\mathrm{x}}} .
\end{aligned}
$$

Here, $K_{\mathrm{ox}}=N K_{\mathrm{m}}$ is the total outside-xylem conductivity and

$$
\frac{1}{K_{\mathrm{x}}}=\frac{1}{\rho} \frac{1}{N} \sum_{i=1}^{N}\left(p_{i}-p_{0}\right)
$$

is the total xylem conductivity. We see that $K_{\mathrm{x}}$ is inversely proportional to the average pressure drop inside the leaf blade.

\subsubsection{The uniform-evaporation approximation}

In a typical leaf network, the number of nodes (vein junctions) is of the order of $N \approx 10^{4}-10^{6}$ (this is supported for instance by our data set from Part II). Because $K_{\mathrm{x}}$ is of the same order of magnitude as $K_{\mathrm{ox}}$ [64], mesophyll conductivity $K_{\mathrm{m}}$ is negligible compared to leaf xylem conductivity $K_{\mathrm{x}}$. Therefore, in this limit, water is subject to a network of $N$ parallel resistors with conductivity $K_{\mathrm{m}}$ connecting petiole and air. By Kirchhoff's laws, the flow will divide equally between the parallel resistors. This means that we can approximate the mesophyll 
conductivities by $K_{\mathrm{m}} \approx 0$ and the mesophyll flows $F_{0 i} \approx \rho / N$.

Because we are interested in the flow on the leaf blade, we are therefore allowed to neglect the outside-xylem pathways and replace them by the boundary condition $\rho_{i}=\rho / N$, see Figure 10.1 (b). This boundary condition can also be understood as modeling uniform evaporation of water from the leaf blade.

\subsubsection{Pressure drop minimization}

Following the preceding arguments, we model the leaf venation network as a weighted flow network. The general mathematics of such networks is reviewed in in Chapter D of the appendix. ${ }^{1}$ The model we use is based on the idea of adjusting the network conductivities such as to achieve minimization of pressure drop in the leaf network (see [70] for the biological underpinnings, and [72] for the initial work in physics; compare also the introduction of this thesis). The objective function is thus (see also (2.12))

$$
\Delta p=\frac{1}{N} \sum_{i=1}^{N}\left(p_{i}-p_{0}\right),
$$

where $p_{i}$ is the hydrostatic pressure at node $i$ in a network of $N+1$ nodes.

\subsubsection{Network cost}

Because the plant has only finite resources to build its network, we need to add a constraint fixing the total cost that venation represents for the plant, similar to the volume-fixing constraint in Chapter 9. Since water comes for free, the major cost is the carbon cost of the xylem vessel cell walls, which we assume to be proportional to cell wall volume $V$. Cell wall width $w$ is assumed to scale with the radius as $w \sim r^{\alpha}$, where $\alpha$ is a parameter. For a single vessel of length $L$, the cost in the thin-wall limit $w / r \ll 1$ is

$$
\begin{aligned}
C & \approx 2 \pi r w L \\
& \sim r^{\alpha+1} L \\
& \sim K^{\frac{\alpha+1}{\beta}} L,
\end{aligned}
$$

where $K$ is the hydraulic conductivity of the vessel that is assumed to scale as $K \sim r^{\beta}$. For Poiseuille flow, $\beta=4$. Introducing the cost parameter $\gamma=\frac{\alpha+1}{\beta}$, the total cost of the network is

$$
C=\sum_{(i j)} K_{i j}^{\gamma} L_{i j}
$$

\footnotetext{
${ }^{1}$ We make a slight change in notation in this chapter, denoting the hydrostatic pressure by the symbol $p$ instead of the general potential $\phi$ used in Chapter D.
} 
where sensible values of $\gamma$ lie in $(0,1)$, defining an economy of scale. This means that it is "cheaper" or more efficient to build one large vessel instead of two smaller ones.

Assuming Poiseuille flow ${ }^{2}(\beta=4)$, the parameter $\gamma$ has a geometrical interpretation for special values. For $\gamma=1 / 2$, the cost is proportional to total xylem volume; for $\gamma=1 / 4$ to total cross-sectional area.

\subsubsection{Equivalence to power dissipation}

We now show that the objective function (10.7) is equivalent to the total viscous power dissipation in the network. The total viscous power dissipation is

$$
\begin{aligned}
P & =\sum_{(i j)} \frac{F_{i j}^{2}}{K_{i j}} \\
& =-\sum_{i, j} p_{i} F_{i j}=\sum_{i>0} p_{i} \rho_{i}-p_{0} \rho \\
& =\rho \Delta p .
\end{aligned}
$$

Combined with (10.6), this is a remarkable result: To first approximation, the leaf network can optimize three physiologically relevant quantities at the same time. First, pressure drop minimization is highly relevant for local function of the leaf, reducing water stress on the cells. Second, leaf hydraulic resistance minimization is highly relevant for global functioning of the whole-plant hydraulic system because leaves provide the bulk of the whole-plant resistance. Third, power dissipation minimization is relevant for the leaf phloem, which exists in the same vascular bundle as the xylem (see also Chapter 9).

Mathematically, the power dissipation function is easiest to handle, therefore we will in the following phrase all optimization problems in the sense of power dissipation minimization.

\subsubsection{The ensemble-averaged objective function}

Using a Lagrange multiplier, we can combine the power dissipation with the network cost as a constraint into one objective function. Being interested in the effects of damage to the network, we consider the damage-averaged version of the power dissipation. The functional is

$$
\xi=\sum_{(i j)} \frac{\left\langle F_{i j}^{2}\right\rangle}{K_{i j}}+\lambda\left(\sum_{(i j)} K_{i j}^{\gamma}-C\right) .
$$

Here, the angle brackets denote an average over the ensemble of all network topologies where a single edge was completely severed. Thus, minimizing

\footnotetext{
${ }^{2}$ Remember that in real leaves this is just a simplifying assumption.
} 
this functional amounts to optimizing the expected power dissipation under the assumption that damage to the leaf network will happen in some edge. By taking partial derivatives with respect to the conductivities, we find for an optimal network the relation

$$
K_{i j}=\frac{\left\langle F_{i j}^{2}\right\rangle^{\frac{1}{1+\gamma}}}{\left(\sum_{(k l)}\left\langle F_{k l}^{2}\right\rangle^{\frac{\gamma}{1+\gamma}}\right)^{\frac{1}{\gamma}}} .
$$

The network perturbation methods developed in Chapter D of the appendix can be used to numerically calculate the expected flow $\left\langle F_{i j}^{2}\right\rangle$. Because the function (10.11) is non-convex (remember that $\gamma \in(0,1)$ ), in general many local minima exist. From evolutionary reasoning we expect that a realistic leaf network would fall into one that is close to the global optimum. Thus, we employ a numerical simulated annealing scheme to refine minima found by iterating the fixed-point equation (10.12) (our method is very similar to the one used in [72]).

One result of numerically optimizing a network using this type of scheme on a triangular lattice was shown in Figure 2.14 in the introduction, demonstrating the remarkable similarity with real leaf networks that the method is able to achieve. In the following, we present some refinements of the method. First, we consider different damage models that represent cases where not the whole network edge is removed but only a part of the conductivity is lost. This could for instance correspond to damage due to plant pathogens such as fungi [50] or embolisms that do not occlude the entire vein $[18,174]$. Then, we increase resolution of the optimizations by choosing particular boundary conditions.

\subsubsection{Effects of partial damage on topology}

In this section we present results for optimal robust transport networks using numerical minimization of the objective function (10.11) for models that include partial damage. All models are defined on a triangular lattice with hexagonal boundary with $E$ edges and $N$ nodes (see Figure 10.2 for examples).

\subsubsection{Two models for partial damage}

Instead of completely severing the veins by setting the conductivity $K_{i j} \rightarrow 0$ for the network edges, we introduce two alternative models that damage the veins only partially. This is inspired by the fact that the xylem cells are equipped with specialized mechanisms to prevent embolisms from spreading (among them the perforation plates, see Figure 2.5), suggesting that embolisms represent an important class of damage $[175,176]$. Another inspiration comes from plant pathogens such as fungi whose hyphae grow inside xylem cells but do not fully occlude them [50]. 
We distinguish two models. The first is multiplicative damage (model $A$ ), where for each damaged edge $(i j)$ we set $K_{i j} \rightarrow(1-\delta) K_{i j}$, with $\delta \in[0,1]$ representing the amount of damage. Thus larger vessels lose more conductivity in absolute terms than smaller vessels. This may represent embolisms due to drought or freeze-thaw cycling where typically a certain fraction of conductivity is lost [175, 176].

The second model is additive damage (model $B$ ), where $K_{i j} \rightarrow K_{i j}-\kappa$, with $\kappa=E^{1 / \gamma} \tilde{\mathcal{K}}$ an absolute damage parameter. In order to render models with different $\gamma$ comparable, we introduced $\tilde{\kappa}$ by normalizing with a typical edge conductivity. This model may represent damage due to plant pathogens that grow inside the xylem but that only reduce conductivity by some fixed value given for instance by hyphae size.

\subsubsection{Results}

(a)

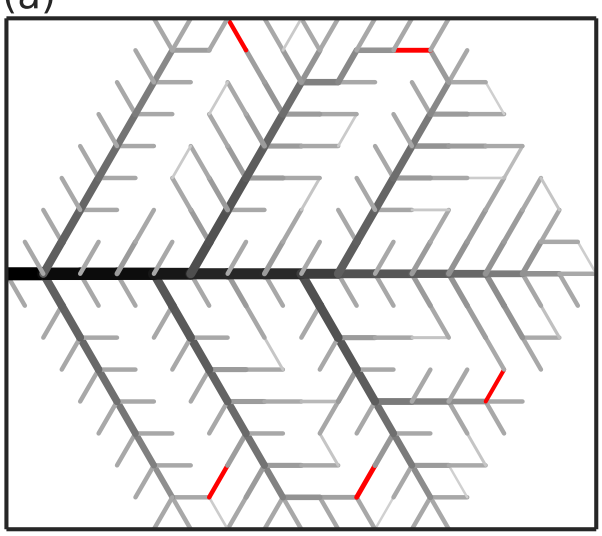

(b)

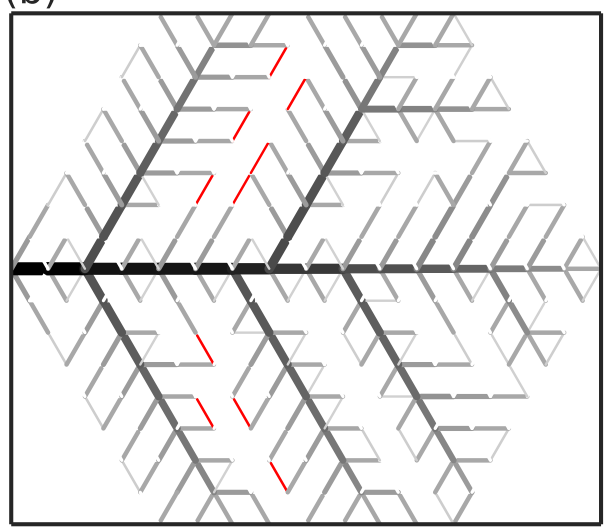

FIGURE 10.2: Formation of first loops in multiplicative and additive damage models at $\gamma=0.5$ on a triangular lattice with $N=217$ nodes and $E=600$ edges. Edge color and width are proportional to $K^{0.15}$. Some anastomoses are marked in red. The leftmost node was chosen as an inlet, all other nodes are uniform outlets. (a) Multiplicative damage, $\delta=0.99$, large anastomoses form first between the secondary veins. (b) Additive damage, $\tilde{\kappa}=0.7$, small anastomoses form first between small veins.

Typical optimal network topology. In both models, the optimal networks contain no loops for small values of the damage parameter. As the damage parameter is increased, loop formation is induced in different ways in the optimal networks depending on the damage model.

For multiplicative damage, large anastomoses form first, connecting the secondary veins. This can be explained by the fact that when the main vein is strongly damaged, a large flow must be rerouted. Since power dissipation is inversely proportional to conductivity, it can be minimized by rerouting through the secondary veins, which possess the largest conductivities apart from the main vein. 
For additive damage, small anastomoses form first, connecting high order veins. The secondary veins remain unconnected. There is no need to build large bypass structures because the damage to the main and secondary veins is negligible. Small veins however are strongly affected such that small, local bypasses need to be built, keeping the cost low.

As the damage parameters are further increased, both optimal network topologies become indistinguishable, as both models become equivalent.

Typical optimal networks for damage models $A$ and $B$ are shown in Figure 10.2 for parameter values above the limit for loop formation.
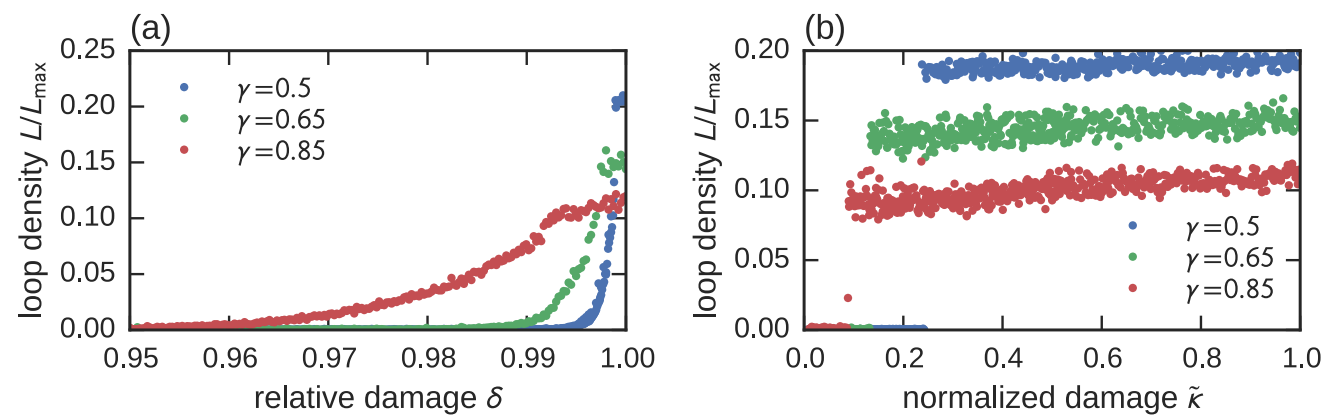

FIGURE 10.3: Density of loops in the network as a function of damage for the two damage models and several values of $\gamma$. Networks had 2437 nodes and 7140 edges. (a) Multiplicative damage $K_{i j} \rightarrow(1-\delta) K_{i j}$. Very large damage $\delta$ is needed before the network starts investing in loops. The number of loops increases smoothly up to a final value. The first loops (small $\delta$ ) are anastomoses between the secondary veins, later $(\delta \approx 1)$ smaller loops form. (b) Additive damage $K_{i j} \rightarrow K_{i j}-E^{1 / \gamma} \tilde{\kappa}$, where $\tilde{\kappa}$ is the typical edge weight. Loop density jumps at a critical $\tilde{\kappa}_{c}$ and then stays approximately constant. The first loops $\left(\tilde{\kappa} \gtrsim \tilde{\kappa}_{\mathcal{c}}\right)$ are many very small ones, later $\left(\tilde{\kappa} \gg \tilde{\kappa}_{c}\right)$ few larger anastomoses form.

How much damage is necessary to induce loops? In order to determine the amount of damage necessary to induce loops, we considered optimal networks in triangular lattice geometry in both models for a range of damage parameters and measured the loop density $L / L_{\max }$, where $L$ is the number of loops in the network and $L_{\max }=E-N+1$ is the maximum possible number of loops in the network according to Euler's formula. To compute $L$, we thresholded the converged network and discarded all edges with conductivity value below the numerical machine accuracy, and then calculated $L=E^{\prime}-N+1$, where $E^{\prime}$ is the number of conducting edges that were not discarded.

The results for both models are shown in Figure 10.3 for some values of $\gamma$. The behavior is strikingly different. In the multiplicative model, a large amount of relative damage is necessary to even make it economical for the network to produce loops (typically, $\delta>0.95$ ). Even then, loop density increases continuously by first adding few large loops connecting large veins and then successively introducing smaller and smaller anastomoses until a characteristic final loop density (depending on $\gamma$ ) is reached. We can distinguish two regimes. First, loop density grows approximately exponentially, then linearly (see Figure 10.3 (a) in 
particular for $\gamma=0.85$ ).

In contrast, the additive model is characterized by a critical damage $\tilde{\kappa}_{c}$ below which there are essentially no loops and above which the loop density grows approximately linearly until it reaches the characteristic final loop density. The critical damage is the conductivity of the smallest veins of which there are many with very similar conductivity. These smallest veins are completely severed at the threshold, triggering the formation of many small anastomoses. As the damage parameter increases, few larger anastomoses are added until the characteristic loop density for given $\gamma$ is reached.

Comparison with the fossil record [68, 113, 177] suggests that early angiosperms already possessed large anastomoses between the secondary veins. If they arose as an adaptation to damage, our models thus suggest that damage due to embolisms may be the dominant driver of natural selection as compared to damage through pathogens.

\subsubsection{Increasing resolution}

So far, we have looked at networks that model the complete venation pattern of a whole leaf. As one wants to increase resolution, computation time becomes prohibitive to exploring higher order structures. For this reason, we now show some ideas that allow us to consider only small parts of the leaf venation pattern by appropriately choosing the lattice on which to optimize and modifying the boundary conditions. These models not only allow us to "zoom in" to small regions of the simulated leaf, but they also provide us with an abstract model for those regions. We consider two main "zoom regimes" and compare to real leaf networks. Each regime is characterized by a particular choice of boundary conditions. In both cases, we switch from the triangular lattice used to model the whole leaf to a square lattice and use the original damage model that completely severs edges with a cost parameter of $\gamma=0.5$. The different lattice is used because we are not interested in approximating a leaf margin anymore, and because the higher order structures of the network are better represented by a square lattice.

\subsubsection{Section adjacent to the main vein}

We consider a $N \times M$ square lattice whose bottom line of $N$ nodes is used to model the main vein. To these nodes we assign the boundary condition $\rho_{i}=-\rho / N, i \in\{1, \ldots N\}$, with $\rho$ the total amount of water transported into the small section of leaf. The other $N-1 \times M$ nodes model uniform evaporation such that for them, $\rho_{j}=\rho /((N-1) M), j \in\{N+1, \ldots, N M\}$. In a real leaf, the main vein's rate of fluid delivery per unit length $\rho_{i}$ depends on the leaf profile (assuming that the same amount of water needs to be delivered per area) such that this model is valid in a region where the distance between leaf margin and main vein remains roughly constant. Such a region exists for many elongated leaves near the center (see, e.g., Figure 2.12). 
(a)

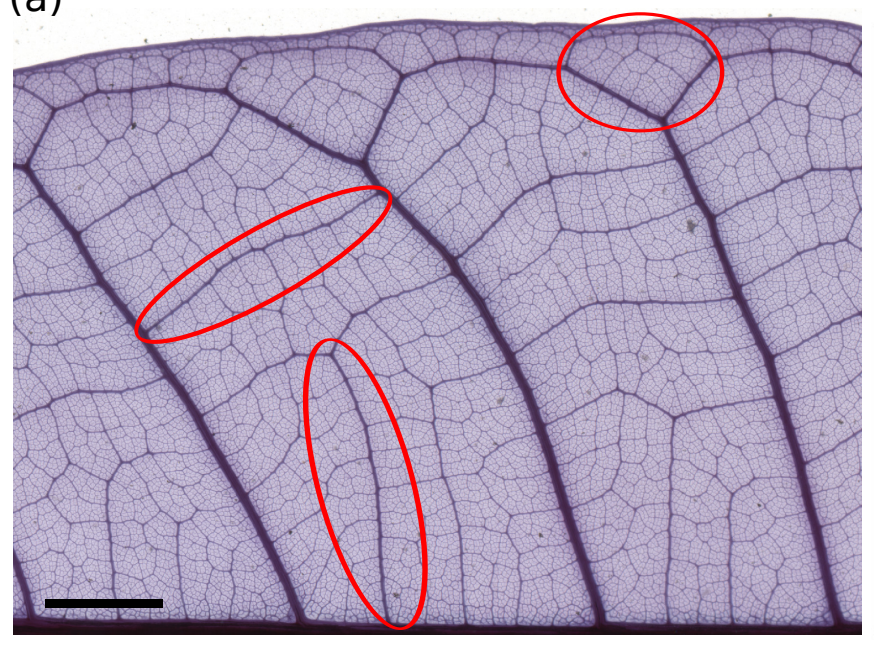

(b)

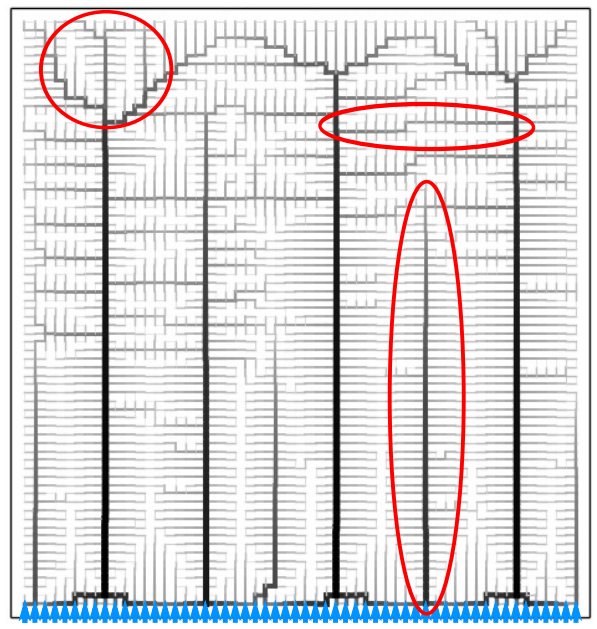

FIGURE 10.4: Boundary condition models reproduce higher order structures found in real leaves near the main vein. (a) Crop of a cleared and stained leaf of Protium paniculatum, showing a typical brochidodromous venation pattern. Three typical sub-patterns are marked in red. The scale bar corresponds to $5 \mathrm{~mm}$. (b) Typical optimal robust transport network on a square lattice with uniform inflow at the bottom (blue arrows). The network reproduces similar sub-patterns as found in the real leaf.

The model clearly reproduces many of the sub-patterns that were not present in the whole-leaf model due to low resolution, in particular those inside large vein-loops formed by the secondary veins (see Figure 10.4). These patterns are commonly found in real leaves with brochidodromous venation such as many of the Burseraceae we analyzed in Part II.

\subsubsection{Section between two large veins}

Again, we consider a $N \times M$ square lattice, but this time the bottom-most and topmost rows are used to model secondary veins. Assuming that both secondaries contribute equally to water delivery into the region between them, we assign them the source net currents $\rho_{i}=-\rho / 2 N, i \in\{1, \ldots 2 N\}$. The nodes in between are uniform sinks with $\rho_{j}=\rho /((N-2) M), j \in\{2 N+1, \ldots, N M\}$. A region with approximately this type of flow pattern exists in real leaves with many long secondary veins, e.g., in some leaves with craspedodromous venation pattern such as hornbeam or birch.

Again, the model reproduces sub-patterns obscured by low resolution or possibly other effects in between the secondary veins of other models. In particular, it reproduces the cross-connections between third order veins the central region between the secondary veins (see Figure 10.5). Because flows in the central region are small, there is little patterning, and veins appear more random.

"Zooming in" even further appears not sensible because the model now becomes self-similar. The region between two third order veins is expected to look roughly like a rescaled version of that between the secondary veins up to a 
(a)

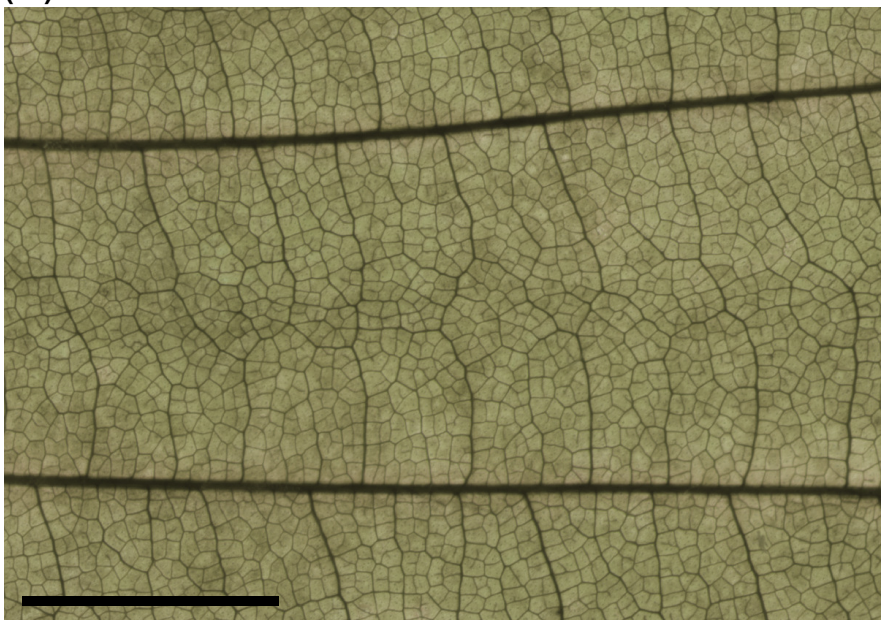

(b)

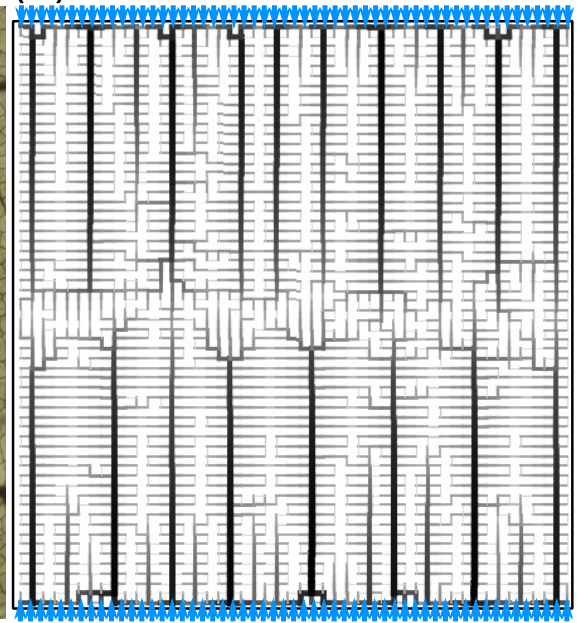

FIGURE 10.5: Boundary condition models reproduce higher order structures found in real leaves between secondaries. (a) Crop of a cleared and stained leaf of Carpinus betulus (European hornbeam). Hornbeams have particularly long, almost parallel secondary veins, approximately satisfying the model conditions. The scale bar corresponds to $5 \mathrm{~mm}$. (b) Typical optimal robust transport network on a square lattice with uniform inflow at the bottom and top (blue arrows). Again, the network reproduces similar sub-patterns as found in the real leaf, in particular in the central region between the two secondaries. (Leaf image (a) courtesy of Torsten Eckstein).

rotation by $90^{\circ}$. The effect of symmetry breaking by the single inlet at the petiole can be neglected. In addition, real leaves tend to possess only up to three welldefined vein orders, and at the scale probed by further increasing the resolution it becomes unclear whether optimization effects still dominate the network. It is likely that effects of mechanical stress on the cells during development take over [93].

\subsubsection{Summary, challenges, and future work}

We have presented a model for damage-robust optimal transport networks. The model minimizes expected power dissipation in a complex flow network under the assumption of damage to the edges. We showed that this optimization is equivalent to both minimizing mean pressure drop on the leaf blade and maximizing the total xylem hydraulic conductivity.

We then extended the model by introducing two different versions of partial damage, one multiplicative the other additive and showed that these lead to different network topologies when increasing the damage parameter. Multiplicatively damaged networks (modeling, e.g., vein embolisms) form large anastomoses first whereas additively damaged networks (modeling, e.g. pathogens) form small anastomoses first. Comparison with the fossil record suggests that embolism damage may be the more important driver of leaf venation evolution.

Next, we introduced two variants of the original damage model with modi- 
fied boundary conditions that represent different regions of the leaf blade. We considered the regions adjacent to the main vein near the center of the lamina and those between long secondary veins. By comparison with real leaves, we showed that the model still applies and reproduces higher order sub-patterns that are found in the real leaves as well.

\subsubsection{Challenges and future work}

Although the evolutionary damage optimization model appears remarkably good at reproducing venation patterns found in real leaves, the global pattern found in craspedodromous leaves poses a challenge. In craspedodromous leaves, the secondary veins do not connect through anastomoses but rather extend further and terminate at the leaf margin, often in serrations.

We have not been able to reproduce this type of venation pattern using an optimal transport network model. It has been suggested that this pattern is correlated to climate, it being prevalent in temperate tree leaves. The pattern is sometimes thought to arise from a principle of parsimony. Because temperate leaves are formed anew each year, they must be produced cheaply and the safety benefit of large anastomoses with respect to cost appears too small [70]. In contrast, non-temperate leaves must remain functional for many years, making a larger investment worthwhile. An example for the craspedodromous pattern is shown in Figure 10.6.

However, we speculate that the craspedodromous venation pattern may be due to developmental constraints arising from folded growth of the lamina within a bud. Such pre-formed leaves are the norm for temperate species and it has been shown that the folds typically occur parallel to the secondary veins [102, 103], thus precluding large (rigid) anastomoses between them because they would prevent efficient unfolding. This hypothesis is further strengthened by observations of tree species that can produce both pre-formed (in a protective bud) and neo-formed (without a bud) leaves. Indeed, in limited studies, pre-formed leaves show craspedodromous venation and neo-formed leaves show brochidodromous venation $[107,108]$.

Thus, an optimal transport network model alone may be inappropriate to explain the transition between the two patterns. Instead, in the future it will

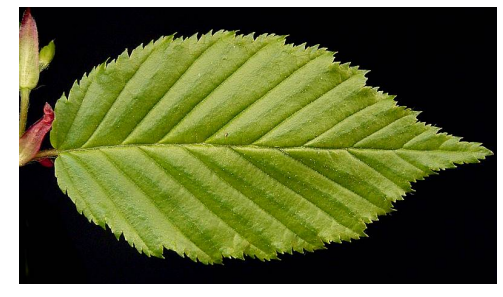

FIGURE 10.6: The craspedodromous venation pattern in Carpinus betulus. Secondary veins terminate in the serrations at the margin. Creases from folded growth can be discerned between the secondary veins. Leaf length is approximately $8-10 \mathrm{~cm}$. We have not been able to reproduce this global pattern. ("Carpinus betulus" by Frank Vincentz, CC BY-SA 3.0 / cropped). be necessary to consider models that can integrate geometrical constraints arising from developmental conditions to the network. In addition, more data is necessary to investigate the correlations between venation pattern, growth habit (pre-formed or neo-formed), and climate. 


\subsection{Auxin canalization models of vein morphogenesis}

Auxin canalization is the most widely accepted theory of vein morphogenesis in plant leaves. It stipulates that a certain phytohormone, auxin, that is produced in the leaf during development and actively transported by certain proteins called PIN is responsible for cell differentiation into vascular tissue. The petiole acts as a sink for auxin. A large number of subtly different models have been proposed [85], some relating vein formation to auxin flux, others to auxin concentration. One fundamental problem in validating these models is the current inability to experimentally measure auxin in the growing leaf; it is only possible to infer transport indirectly [89]. In addition, many models make similar predictions. It must be stressed, though, that the fact that auxin is responsible for vascular differentiation is uncontested and can be clearly demonstrated by experiment [88].

One problem with many models of auxin canalization is explaining the formation of vein loops [178, 179]. Modelers have been forced to resort to either introducing new hypothesized chemicals ("flux-bifurcators") [99] or to manually add and remove auxin sources at specific times [97]. Either way, the resulting networks do not look particularly realistic, or contain only one or two loops that are thought to model the first loops that form in the growing leaf. A more abstract approach was used in [180], producing very realistic networks. However, the model does not use a feedback mechanism but rather a set of geometric rules because it was designed to produce leaf networks for use in computer generated images. The way the model employs stochasticity however suggests its usefulness in explaining venation patterns.

Our aims for this section are not to provide yet another detailed and finetuned model for vein morphogenesis. Instead, we introduce a simple flux-based model for auxin canalization, neglecting even polar auxin transport by PIN. Recently, dynamic expression of auxin-related genes was reported in live imaging experiments [91]. It was demonstrated that expression levels of both PIN proteins and a certain vascular commitment marker show transient behavior, leading us to hypothesize fluctuations in auxin flow during morphogenesis. Thus, we introduce random but spatially correlated dynamics of auxin sources in the flow network.

In addition, we introduce an empirical term to the model that represents how veins tend to grow-large veins grow first, smaller veins grow later [86]. We show that this stochastic model (with no need for hypothetical new chemicals or manual placement of auxin sources) reproduces low order venation patterns of real leaves remarkably well.

Our model is not meant to be biologically accurate but rather to serve as a proof-of-concept. The emergence of reticulate venation patterns does not rely on the often emphasized polar auxin transport by PIN and can be explained by stochastic auxin sources coupled to growth. We emphasize that no fine-tuning of parameters is necessary to achieve natural-looking networks.

We also show that by varying the correlation range (i.e., the spatial extent of 

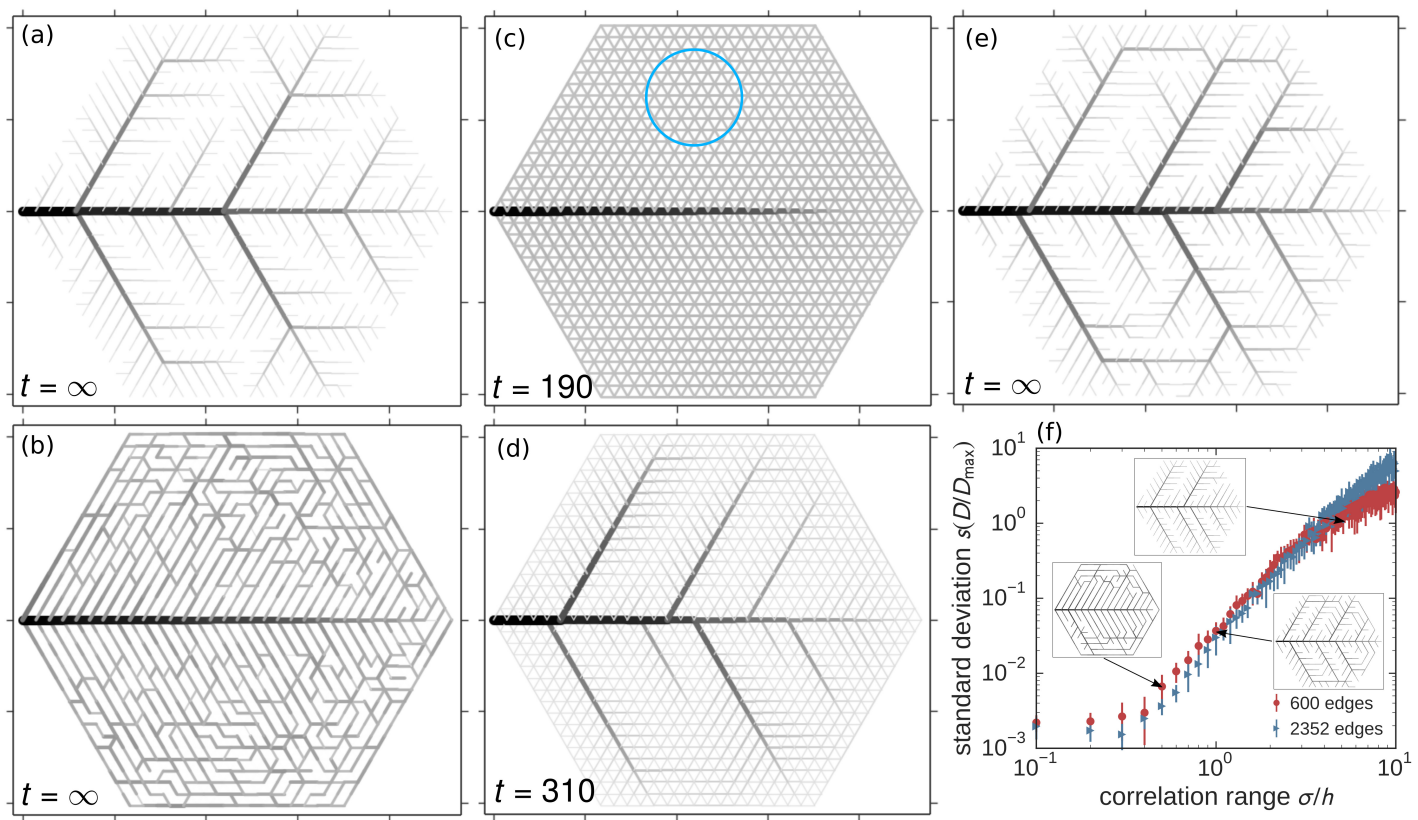

FIGURE 10.7: Typical results from auxin canalization models on a triangular grid. The petiole (leftmost node) acts as a net auxin sink, all networks have 817 vertices and 2353 edges and $\tau=50, \kappa=1$. Edge thickness and color represent the facilitated diffusion coefficient between cells $D_{i j}$. The differential equation was solved using a forward Euler scheme with $d t=0.1$. (a) Steady state network without any fluctuating sources, $\rho_{i} \equiv$ const. The network is a topological tree but shows main vein as well as secondaries. (b) Steady state network with uncorrelated fluctuations, each node is a fluctuating source with zero spatial extent, $\sigma \rightarrow 0$. There is a main vein, but the remaining topology is not leaf-like. (c-e) Time series of network dynamics with spatially correlated fluctuations. We chose $\sigma=3.8 h$, where $h$ is the bond length (blue circle in (c) for one source/sink). There is one auxin source/sink centered at each node with frequencies and shifts chosen uniformly at random, $\omega_{j} \in[0,10], \theta_{j} \in[0,10]$. The main vein forms first, followed by secondaries and higher order veins. The steady state network (e) shows the reticulation pattern typical for real brochidodromous leaves. (f) The standard deviation of the normalized diffusion coefficient distribution shows a continuous transition between non-hierarchical, highly reticulate topologies $(\sigma \ll h)$ and hierarchical, non-reticulate topologies $(\sigma \gg h)$. Results are shown for two system sizes, typical networks are shown for $\sigma / h=0.5,1,5$ in the system with 600 edges.

auxin sources and sinks) it is possible to obtain a continuous family of venation topologies whose individual members differ in loop density and the amount of hierarchical organization.

\subsubsection{Correlated fluctuations lead to realistic networks}

The model for auxin canalization we use is based on the simple model described in [97]. We represent the cells of the growing leaf lamina as nodes of a planar network. Between adjacent cells, there is auxin flow according to

$$
F_{i j}=D_{i j}\left(c_{i}-c_{j}\right)
$$


where $F_{i j}$ is the flow of auxin between cells $i$ and $j, D_{i j}$ is an effective facilitated diffusion constant, and $c_{j}$ is auxin concentration in cell $j$. Thus, this is a linear flow law (see Chapter D of the appendix) for the auxin concentrations.

The network evolves according to a positive feedback mechanism governing the magnitude of the facilitated diffusion constants. This is the core of the canalization hypothesis. High flow increases facilitated diffusion, leading to higher flow; unused channels die out. A large $D_{i j}$ is then interpreted as corresponding to vein precursor tissue. In our model, the diffusion constants evolve according to the nondimensional equation

$$
\frac{d D_{i j}}{d t}=\left(F_{i j}^{2}\right)^{\frac{2}{3}}-D_{i j}+\kappa e^{-t / \tau}
$$

This dynamical system contains three competing terms. The first term can be interpreted as positive feedback to the flow magnitude. The precise form of the nonlinearity is not crucial for the results. Because our model is a simple proof-of-concept, we focus on one particular flow-feedback relationship amongst many that produce realistic looking networks. The second term models decay of unused channels. If it dominates, flow is small and the channel vanishes. The third term models growth. The initial conditions are chosen such that it dominates at the beginning (by an appropriate value of $\kappa$ ) as growth starts out uniform. As the term decays with the time scale $\tau$, the feedback mechanism is able to take over first where flow is largest and consequently the largest veins will grow. As time progresses, the feedback mechanism successively applies to smaller and smaller veins until convergence. This corresponds to the empirical fact that during development, veins appear in order of their relative thickness (compare Figure 2.17). It must be emphasized that the third term in (10.14) is purely phenomenological but produces very realistic networks. This type of growth term has a similar effect on the network growth dynamics as the simulated annealing technique used in [72] and in the previous section has on optimization dynamics.

Cells can be producers or sinks for auxin. Our model assumes spatially correlated, fluctuating sources and sinks. The nondimensional sources and sinks are modeled by

$$
\begin{aligned}
\rho_{i} & =\sum_{j=1}^{N} e^{-\left\|\mathbf{x}_{i}-\mathbf{x}_{j}\right\|^{2} /\left(2 \sigma^{2}\right)} \cos \left(\omega_{j}\left(t-\theta_{j}\right)\right), \quad i>0 \\
\rho_{0} & =-\sum_{i=1}^{N} \rho_{i} .
\end{aligned}
$$

Here, the node 0 corresponds to the petiole, which acts as a sink for the net produced auxin. All other nodes nodes are sources or sinks with spatial extent (correlation range) $\sigma$ and centered at position $\mathbf{x}_{j}$. They alternate between 
source and sink behavior according to the frequencies $\omega_{j}$ and shifts $\theta_{j}$. Simple alternatives to this model include no fluctuations ( $\rho_{i} \equiv$ const), or spatially uncorrelated fluctuations $\left(\rho_{i} \sim \cos \left(\omega_{i}\left(t-\theta_{i}\right)\right)\right.$.

Typical simulation results are shown in Figure 10.7. If fluctuations are absent, the steady state network is a topological tree but still shows the characteristic main and secondary veins. If fluctuations are spatially uncorrelated, a main vein forms but the rest of the topology is not leaf-like (the topology resembles the fluctuating sink models described in $[72,114])$. Only if fluctuations are spatially correlated do characteristic, realistic anastomoses between the secondary and higher order veins form that resemble the results from the damage optimization models from the preceding section and [72], as well as real leaf topologies.

It should be noted that the values of the fluctuation frequencies $\omega_{j}$ and time shifts $\theta_{j}$ need no fine-tuning. The behavior shown in Figure 10.7 is robust over one to two orders of magnitude in the $\omega_{j}$.

\subsubsection{A continuous transition between network topologies}

There exists a continuous transition between strongly differing steady-state topologies as the spatial extent of correlated fluctuations varies in comparison with the edge length $h$ (i.e., cell size). This is quantified by the standard deviation $s\left(D / D_{\max }\right)$ of the normalized steady state distribution of non-zero effective diffusion coefficients. ${ }^{3}$ For $\sigma=0$, the distribution is close to Gaussian and the standard deviation is small. For $\sigma \gg h$, the network becomes more fractal-like and the conductivity distribution is long-tailed. This is shown in Figure 10.7 (f). The transition point where the network begins to "feel" the spatial correlations appears to be at $\sigma \approx h / 2$.

As $\sigma$ becomes comparable with the lattice size, the loops in the network vanish. In general, the loop density decreases as $\sigma$ increases.

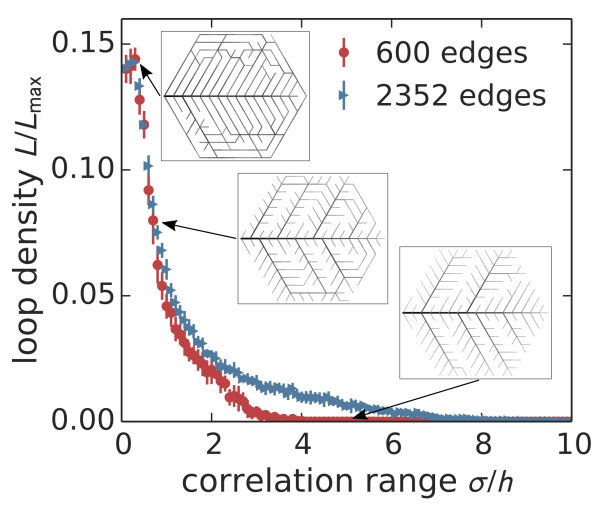

FIGURE 10.8: Loop density shows a continuous transition between topologies as the correlation range varies. The inset shows typical steady state networks at $\sigma / h=$ $0.5,1,5$. Data are taken from the same networks as in Figure 10.7 (f). This behavior is shown in Figure 10.8.

We emphasize that the steady state solutions of (10.14) for $t \rightarrow \infty$ are formally very similar to the minima (10.12) of the evolutionary optimization functional (10.11) (for appropriate choice of boundary conditions, averaging ensemble, and cost parameter $\gamma$, if such a choice is possible; in the case of no fluctuations they can be immediately seen to coincide). For instance, the fluctuating sink model from [72] corresponds to our model with $\sigma=0$. Thus, there is a direct link between dynamical models such as the one explored in this section and

\footnotetext{
${ }^{3}$ All conductivities below a fixed threshold are assumed to have converged to zero and are removed from the distribution.
} 
optimization models such as the one presented in the preceding section. Similar models have been considered in the past finding similar connections where local adaptation rules globally optimize some functional [181, 182], but these were not focused on the peculiarities of leaves. The work [181] found a transition between non-reticulate and reticulate networks as fluctuations are gradually switched on, but apparently missed the transition between different types of topologies that we have pointed out here. We note that the dynamical system (10.14) minimizes pressure drop (or equivalently power dissipation) during the dynamics [181].

\subsubsection{Summary and future work}

In this section, we presented an extremely simple dynamical model for auxin canalization that neglects polar transport by PIN proteins. The model represents the way auxin flows and leaf tissue dynamically adapts during growth and morphogenesis of the leaf blade. We showed that spatially correlated source and sink strength fluctuations during growth, which we modeled using an empirical term in the dynamical equation, lead to realistic, leaf-like topologies with main vein, secondary veins and characteristic anastomoses. This may be related to a connection between optimal flow networks and steady states of dynamical systems such as those in this and the preceding section.

In addition, we uncovered a transition between different reticulate topologies as one moves from spatially uncorrelated to spatially correlated fluctuations that had been previously missed. It is precisely the spatial correlations that lead to realistic leaf networks, and dynamical fluctuations have been observed in leaves during morphogenesis. It is remarkable that a simple local feedback rule such as (10.14) coupled to growth leads to a pattern that optimizes a global functional. Thus, natural selection has had it easy: instead of encoding complicated network patterns, very simple morphogenetic mechanisms suffice to produce highly optimized structures "automatically" in a self-organized fashion.

Our model is extremely simple, and there is no direct justification for the growth term in our dynamical equation. Thus, future work needs to focus on both justifying the growth term (possibly modifying it) and performing simulations on growing networks. Additionally, it would be worthwhile to further investigate connections between optimal transport networks and dynamical systems, possibly finding a "dictionary" allowing one, e.g., given a dynamical system and its steady states, to construct the corresponding optimization functional with the same minima, and vice-versa. It would also be interesting to incorporate more realistic auxin dynamics, i.e., polar transport by PIN proteins. 



\section{Final Summary}

In this final chapter we briefly summarize all the work in this thesis once more, put it into perspective, and discuss in which ways the goals set in the introduction have been met.

\subsection{Biology of Leaves and Leaf Venation}

In Part I, we discussed aspects of function, development, and evolution of the dicotyledonous plant leaf with special focus on physical modeling. We gave a description of the cohesion-tension theory of water movement through the plant. We discussed the xylem as water transport tissue as well as the mathematics behind CT theory in terms of local (vein level) and global (whole tree level) models of hydraulic resistors. Next, we discussed the Münch mechanism of active, osmotic-gradient driven photoassimilate transport through the phloem. We discussed aspects of leaf shape and presented a global view of the leaf venation network and its hydraulics. We especially focused on the fact that the venation network appears to be highly optimized by evolution for its task. In the following section, we discussed development of the dicot and monocot leaf from the shoot apical meristem as well as vein morphogenesis and auxin canalization theory.

\subsection{Topological Phenotypes in Leaf Vascular Networks}

In Part II, we systematically analyzed the topological and geometrical traits of a set of 186 leaves and leaflets, mainly from the Burseraceae family. Our work is the first study of its kind. For each leaf we calculated a "fingerprint" consisting of five well-studied geometrical and three novel topological traits. The resulting feature vectors constituting the phenotypic space of leaf venation were then analyzed by Principal Component Analysis and Factor Analysis, revealing geometry and topology as complementary dimensions.

Our results demonstrate that hierarchical topology consists an entirely new dimension in the phenotypic space of leaf venation that is approximately orthogonal, in the sense of PCA and FA, to geometry. It contains a large amount of additional information about the venation network as evidenced by its ability to significantly improve specimen identification from fragments. Such identification tasks may be important especially when dealing with leaf fossils or leaf litter where only small parts are available. 
Additionally, we showed how this new phenotype may be explained by significant amounts of noise affecting vein morphogenesis at different stages. Our work shows that stochasticity may be a crucial ingredient for the description of vein morphogenesis.

\subsection{Network Modeling}

In Part III, we studied several models of venation optimization and morphogenesis in diverse settings. We started from simple, one-dimensional pine needles and moved on to two-dimensional leaves.

\subsubsection{Optimal Geometry of Pine Needle Phloem}

We studied a simple model of nutrient transport in the phloem of pine needles and compared its predictions to direct measurements of phloem geometry in four conifer species. The model is based on the optimization of viscous power dissipation during flow.

Analytically optimizing power dissipation in the needle under the constraint of constant total volume leads to the prediction that the total cross-sectional area of all phloem cells should scale as $A(x) \sim x^{1 / 2}$ as a function of distance from the needle tip. This scaling is roughly observed in the experimental data.

\subsubsection{Robust Optimal Transport Networks}

We presented a model for damage-robust optimal transport networks. The model minimizes expected power dissipation in an ensemble of complex flow networks under the assumption of damage to the edges. We showed that this optimization is equivalent to both minimizing mean pressure drop on the leaf blade and maximizing the total xylem hydraulic conductivity.

We then extended the model by introducing two different versions of partial damage, one multiplicative the other additive and showed that these lead to different network topologies when increasing the damage parameter. Multiplicatively damaged networks (modeling e.g. vein embolisms) form large anastomoses first whereas additively damaged networks (modeling e.g. pathogens) form small anastomoses first. Comparison with the fossil record suggests that embolism damage may be the more important driver of leaf venation evolution.

Next, we introduced two variants of the original damage model with modified boundary conditions that represent different regions of the leaf blade. We considered the regions adjacent to the main vein near the center of the lamina and those between long secondary veins. By comparison with real leaves, we showed that the model still applies and reproduces higher order sub-patterns that are found in real leaves as well. 


\subsubsection{Auxin Canalization}

Finally, we presented a simple dynamical model for auxin canalization, neglecting polar transport by PIN proteins. The model represents the way auxin moves and leaf tissue dynamically adapts during growth and morphogenesis of the leaf blade. We showed that spatially correlated source and sink strength fluctuations during growth lead to realistic, leaf-like topologies with main vein, secondary veins and characteristic anastomoses. This may be related to a connection between optimal flow networks and steady states of the dynamical model.

In addition, we uncovered a transition between different reticulate topologies as one changes the spatial correlation range of fluctuations. It is precisely the spatial correlations that lead to realistic leaf networks, and dynamical fluctuations have been observed in leaves during morphogenesis.

\subsection{Have we achieved our aims?}

In the introduction, we described our aims very broadly as finding an answer to the questions "What is the connection between geometry and topology of leaf networks?", and "What is the connection between the physics of evolution and development of leaf networks?".

Clearly, we were only able to give partial answers. We showed that empirically, topology and geometry of leaf venation networks are approximately independent (in the sense of PCA), and that a very simple empirical model can explain the variation in topology as measured by our metrics as effects of characteristic stochasticity during development. Additionally, we showed that simple models of evolution and development can produce realistic leaf networks, and that they are linked because the critical points of the evolutionary optimization functional appear formally equivalent to the steady states of the developmental model.

Thus, much work remains to be done, and we have given several examples for possible follow-up research to each of our topics in their respective chapters. Additionally, it appears worthwhile to use the tools developed in Part II for quantitative analysis of the venation patterns generated from the models in Part III, thus connecting the two main avenues followed in this thesis.

We hope that the nesting software package developed for the work in Part II will stimulate other workers to include topology of venation networks in their research. 

Appendix 



\section{A Statistical Techniques for Phenotyping}

The evaluation of topological and geometrical information contained in leaf venation networks necessitates the use of tools from statistics. Most of the algorithms we use can be grouped under the umbrella of machine learning, the study of pattern recognition, classification, and artificial learning. There exists no overarching theory of machine learning; instead it consists of a collection of related algorithms, tools and methodologies which can be used to ask questions of similarity (how similar are these two leaf networks?), classification (which leaf does this fragment belong to?) or validation. The tools from machine learning are datadriven and aimed at mimicking and enhancing human information processing capabilities [183]; they can be used to make sense of large amounts of data for which no or little a priori intuition exists. Therefore, they are ubiquitous in the fields of image analysis, data mining, statistics, and artificial intelligence [184186]. Many different approaches exist. Here, we will mainly follow the notation and algorithms in [183], many of which were implemented in scikit-learn, a machine learning package for the Python programming language [187]. This package was used to analyze the data set and obtain the results presented in Chapter 5. We will not give a comprehensive overview of available techniques or present any novel insights but rather focus on a concise summary of wellestablished methods which we have found to be useful in the study of leaf venation networks.

The rest of this chapter is organized as follows. First, we present a refresher on basic statistics, including the Gaussian distribution, statistical tests, and the notion of likelihood. Then, we present validation techniques for statistical methods. Further, we give a short introduction to information theory and distance measures between probability distributions. Following this, we define data and covariance matrices, and present algorithms extracting information contained therein and providing dimensional reduction (Principal Component Analysis and Factor Analysis). We proceed to discuss clustering algorithms, unsupervised machine learning techniques that detect structure in the data not directly contained in the covariance matrix. Finally, we discuss discriminant analysis, a supervised machine learning technique that, once trained on known data, allows to predict class membership of new data as well as provide a means of dimensional reduction.

\section{A.1 Statistics}

Statistics is the basic tool of any data scientist. It allows one to make quantitative statements about the validity of hypotheses about the data. Since mathemati- 
cal statistics underlies all of the machine learning techniques described in the later sections, and is important in its own right, in this section we will give a short overview over some basic ideas that will be used later both as the basis of machine learning and for its evaluation. Our approach for the most part follows [183] for specific machine learning terminology and is mostly thought of as a refresher. Many good textbooks for general statistics exist, e.g., [188].

\section{A.1.1 The Gaussian distribution}

Arguably the most important probability distribution in all of science and mathematics because any mean of i.i.d. random variables converges to one in probability, the multivariate Gaussian distribution with mean $\mathbf{m}$ and covariance matrix $\Sigma$ is defined as

$$
p(\mathbf{x})=\mathcal{N}(\mathbf{x} \mid \mathbf{m}, \Sigma)=\frac{1}{\sqrt{\operatorname{det}(2 \pi \Sigma)}} \exp \left(-\frac{1}{2}(\mathbf{x}-\mathbf{m})^{T} \Sigma^{-1}(\mathbf{x}-\mathbf{m})\right) .
$$

We note that any projection onto a lower dimensional subspace of a multivariate Gaussian distribution is again a Gaussian.

\section{A.1.2 Statistical tests}

Statistical tests are used as a means to quantitatively discriminate between two hypothesis for a given sample, called the null hypothesis $H_{0}$ and the alternative hypothesis $H_{A}$. A statistic $s$ is some number calculated from the sample for which the conditional distribution $p\left(s \mid H_{0}\right)$ is known or can be estimated. After defining a significance level $\alpha$, it can be used to decide whether or not to reject the null hypothesis given the observed $s_{\text {obs }}$ (one often chooses $p\left(s \geq s_{\text {obs }} \mid H_{0}\right) \leq \alpha$ as the condition) and accept the alternative hypothesis instead.

In the following we describe some simple tests that are we found useful in the study of leaf phenotypes. All of them are well-known.

\section{A.1.2.1 Student's $t$ test}

Student's $t$ test can be used to test whether the means of two random samples $\mu_{1}, \mu_{2}$ coming from Gaussian distributions with equal variance $\sigma$ differ significantly. If the variances are unequal, Welch's test must be used. For the sake of simplicity, we only describe the classical Student's test for equal size samples. One calculates the statistic

$$
t=\sqrt{\frac{n}{2}} \frac{\mu_{1}-\mu_{2}}{s}
$$

where $s=\sqrt{\left(\sigma_{1}^{2}+\sigma_{2}^{2}\right) / 2}$ and $\sigma_{1,2}^{2}$ are unbiased estimators of the sample variances and $n$ is the sample size. If the null hypothesis $H_{0}: \mu_{1}=\mu_{2}$ is true, (A.2) 
now follows a $t$ distribution with $2 n-2$ degrees of freedom.

\section{A.1.2.2 Kolmogorov-Smirnoff test}

The Kolmogorov-Smirnoff (KS) test is used to decide whether two samples are drawn from the same underlying probability distribution. Given the (empirical) sample distributions $p_{1,2}(X=x)$ one calculates the (empirical) cumulative distributions $F_{1,2}(x)=p_{1,2}(X \leq x)$. The $\mathrm{KS}$ statistic is then

$$
D_{\mathrm{KS}}=\sup _{x}\left|F_{1}(x)-F_{2}(x)\right|,
$$

the greatest distance between the cumulative distribution curves. Under the null hypothesis $H_{0}: F_{1}=F_{2}$, the statistic asymptotically follows a distribution which is analytically known. The KS statistic (A.3) can also be used as a simple way of defining a metric on the space of probability distributions (see Section A.2.2).

\section{A.1.2.3 Likelihood and likelihood-ratio test}

Often, one is interested in fitting a statistical model to a sample, e.g., a Gaussian. This means selecting a set of model parameters (e.g., mean and variance) which fit the sample. We define the likelihood of a set of parameters $\theta$ given the sample $x$ as

$$
L(\theta \mid x)=p(x \mid \theta),
$$

i.e., the probability of observing the data given the model and the parameters. If all observations are independent, the likelihood factorizes into

$$
L\left(\theta \mid\left\{x_{1}, \ldots x_{n}\right\}\right)=\prod_{i=1}^{n} p\left(x_{i} \mid \theta\right) .
$$

Because such products are often difficult to handle, one defines the log-likelihood

$$
\Lambda(\theta \mid x)=\log L(\theta \mid x) .
$$

The log-likelihood can be used for discriminating between hypotheses for the parameters. Let $\Theta$ be some subset of the parameter space, then the hypotheses $H_{0}: \theta \in \Theta$ and $H_{A}: \theta \in \Theta^{c}$ can be discriminated by calculating the loglikelihood ratio

$$
D=-2 \Lambda_{0}+2 \Lambda_{A}
$$

Asymptotically, this statistic follows a $\chi^{2}$ distribution with degrees of freedom equal to the difference in dimensionality between $\Theta^{c}$ and $\Theta$. It is useful for testing whether the sample can be modeled using a special case of a more complicated model (i.e., by fixing some parameters). 


\section{A.1.3 Validation techniques}

Predictive statistical methods such as regression or supervised learning algorithms like discriminant analysis (see section A.6.1) need to be validated to guard against overfitting and assess prediction quality. In this section we review some basic techniques aimed at classification algorithms (i.e., the predictions are taken to be discrete classes).

\section{A.1.3.1 Confusion matrix}

Once an algorithm has been trained to classify some data, its performance can be quantified by looking at the classification results on some other, known data. Often, a single data set is split into a training set and a test set. The predictions of a binary classifier (operating on the two classes "positive" and "negative") can be assessed using the confusion matrix:

\begin{tabular}{|c|c|}
\hline true positives (TP) & false positives (FP) \\
\hline false negatives (FN) & true negatives (TN) \\
\hline
\end{tabular}

The confusion matrix can be extended to multi-class classification. More metrics can be defined using its entries. The accuracy $(\mathrm{TP}+\mathrm{TN}) /(\mathrm{TP}+\mathrm{TN}+\mathrm{FP}+\mathrm{FN})$ measures the probability of correctly classifying a sample. This definition can also serve as the generalization to the multi-class case. Similarly, one defines the sensitivity, or true positive rate $\mathrm{TP} /(\mathrm{TP}+\mathrm{FN})$, the specificity, or true negative rate $\mathrm{TN} /(\mathrm{FP}+\mathrm{TN})$ and the fall-out, or false positive rate $\mathrm{FP} /(\mathrm{FP}+\mathrm{TN})=$ 1 - specificity. There exist two ways of generalizing these metrics to multiclass classifiers. First, the confusion matrix is calculated for each class in a one-vs-rest fashion, lumping all other classes together and comparing. Then, macro-averaging computes the metric for each class individually and then takes the average (possibly weighted by the class population). Alternatively, microaveraging first takes the averages of each entry in the confusion matrix, and then uses these to calculate the metrics.

\section{A.1.3.2 Receiver Operating Characteristic}

The Receiver Operating Characteristic (ROC) is obtained by plotting the true positive rate against the false positive rate. For classifiers that depend on some parameter, each point on the plot corresponds to one parameter value. In machine learning one often lets each point correspond to a subset of the sample for which some confidence score (measuring how "certain" the algorithm is in its classification) is above a certain threshold. The details can depend on the algorithm in question. In both cases we obtain a curve between $(0,0)$ and $(1,1)$.

A perfect classifier would end up in at the point $(0,1)$ with only true positives and zero false positives. A random classifier would lie on the diagonal, and a classifier that is worse than random occupies a point below the diagonal. 
To assess a machine learning algorithm one calculates the area under the ROC curve (ROC AUC), with a value of 1 corresponding to a perfect classifier, and a value of $1 / 2$ to a random classifier.

Multi-class generalizations of the ROC AUC are possible via micro- or macro-averaging.

\section{A.1.3.3 Cross validation}

Given a data set $T$, the performance of a classifier can be estimated by randomly partitioning $T=$ $T_{\text {train }} \cup T_{\text {test }}$. Typically, the training set is chosen larger than the test set. The classifier is trained using the data in $T_{\text {train }}$ and tested on $T_{\text {test }}$ by calculating metrics such as accuracy or ROC AUC. This method can be extended to $k$-fold cross validation by partitioning $T$ not into two but $k$ equally sized subsets $\left\{T_{i}\right\}_{i=1}^{k}$. The algorithm is trained for each $T \backslash T_{i}$ and then tested on $T_{i}$. The performance metrics are then averaged, leading to a more robust estimate of performance. A popular choice is $k=10$. A further increase in robustness can be achieved by stratified $k$-fold cross validation. In this method, the relative number of elements from each class is kept roughly constant in each fold, giving a more realistic representation of the data.

\section{A.2 Information theory and statistical distances}

\section{A.2.1 Entropy}

Statistical information theory is based on the seminal work by Shannon [189] who was interested in quantifying the capacity of transmission channels and devising optimal encoding schemes. However, his ideas have found applications in diverse other settings. Given a discrete probability distribution $P_{X}(x)$ for a random variable $X$, Shannon defined the entropy

$$
H(X)=-\sum_{x} P_{X}(x) \log P_{X}(x),
$$

where the sum runs over all events in the probability space. The entropy of a probability distribution $P_{X}(x)$ is the expected information $I(X)=-\log P_{X}(x)$ which is the unique function that satisfies the axioms

1. $I(p) \geq 0$ (non-negativity)

2. $I(1)=0$ (events that always occur do not carry information) 
3. $I(p q)=I(p)+I(q)$ (the information carried by independent events is additive).

Notice the structural similarity to the thermodynamic Gibbs entropy of a statistical mechanical system. The entropy $H(X)$ is often interpreted as the amount of uncertainty or disorder in a probability distribution. An alternative interpretation is as average surprise. A certain event elicits no surprise $(-\log 1=0)$, an impossible event leads to infinite surprise $(-\log 0=\infty)$.

One defines the conditional entropy as

$$
\begin{aligned}
H(X \mid Y) & =H(X Y)-H(Y)=\sum_{y} P_{Y}(y) H(X \mid Y=y) \\
& =\sum_{x, y} P_{X Y}(x, y) \log \frac{P_{Y}(y)}{P_{X Y}(x, y)}
\end{aligned}
$$

where $H(X Y)$ is the entropy of the joint distribution $P_{X Y}(x, y)$ with marginals $P_{X}(x)=\sum_{y} P_{X Y}(x, y)$ and $P_{Y}(y)$. The conditional entropy is $H(X \mid Y)=0$ if and only if $X$ is entirely determined by $Y$ and $H(X \mid Y)=H(X)$ if and only if $X$ and $Y$ are independent (i.e., $P_{X Y}(x, y)=P_{X}(x) P_{Y}(y)$ ).

The information entropy $H(X)$ is the basis of several measures used to characterize the amount with which probability distributions differ from one another.

\section{A.2.2 Distance measures}

In this section we describe several methods allowing one to define a distance (metric) on the space of probability distributions. Some of them are derived from information theory, others follow different ideas. In general, they can be separated into metrics that use the probability distributions directly and those that use the cumulative distribution. Metrics that use the cumulative distribution have the benefit of not relying on binning if the samples are drawn from continuous distributions.

\section{A.2.2.1 Kullback-Leibler divergence}

The Kullback-Leibler divergence of the distribution $P$ with respect to the distribution $Q$ is

$$
D_{\mathrm{KL}}(P \| Q)=-\sum_{x} P(x) \log \frac{P(x)}{Q(x)} .
$$

Intuitively, $D_{\mathrm{KL}}(P \| Q)$ measures the additional number of bits needed to encode samples from $P$ using a code optimized for $Q$ [190]. It is not symmetric and therefore not a metric in the mathematical sense. In order to circumvent this 
problem, one often uses a symmetrized version

$$
D_{\mathrm{KL}}^{\prime}(P \| Q)=D_{\mathrm{KL}}(P \| Q)+D_{\mathrm{KL}}(Q \| P),
$$

but this still does not satisfy the triangle inequality. However, both standard and symmetric Kullback-Leibler divergence vanish if and only if $P$ and $Q$ are identical, and are non-negative.

The entropy $H(P)$ can be interpreted as the special Kullback-Leibler divergence $D_{\mathrm{KL}}(P \| 1 / n)=H(P)-\log n$, where $n$ is the cardinality of the event set and $Q(x)=1 / n$ is the uniform distribution.

\section{A.2.2.2 Mutual information}

The mutual information between two random variables $X, Y$ with distributions $P_{X}, P_{Y}$ is defined as

$$
\begin{aligned}
I(X: Y) & =H(X)+H(Y)-H(X Y) \\
& =H(X)-H(X \mid Y)=H(Y)-H(Y \mid X) \\
& =D_{K L}\left(P_{X Y} \| P_{X} P_{Y}\right) .
\end{aligned}
$$

It is non-negative, symmetric and captures any statistical dependency between the random variables $X$ and $Y$ (not just linear relationships like the correlation coefficient). This can be seen from its expression as the Kullback-Leibler divergence of the joint distribution $P_{X Y}$ with respect to the product distribution $P_{X} P_{Y}$, the mutual information vanishes if and only if $X$ and $Y$ are independent.

The mutual information can be turned into a true metric in various ways [191], one of which is the variation of information

$$
d(X, Y)=H(X Y)-I(X: Y),
$$

which satisfies non-negativity and the triangle inequality, and vanishes if and only if $X=Y$.

\section{A.2.2.3 Jensen-Shannon divergence}

The Jensen-Shannon divergence [192] between two random variables $X, Y$ with distributions $P, Q$ is defined as

$$
\begin{aligned}
J(P \| Q) & =\frac{1}{2} D_{\mathrm{KL}}(P \| M)+\frac{1}{2} D_{\mathrm{KL}}(M \| Q) \\
& =H\left(\frac{1}{2}(X+Y)\right)-\frac{1}{2}(H(X)+H(Y)),
\end{aligned}
$$

where $M=(P+Q) / 2$ is the symmetric mixture distribution of $P$ and $Q . J(P \| Q)$ is non-negative, bounded from above by $\log 2$, vanishes if and only if $P=Q$, 
and its square root satisfies the triangle inequality, making $\sqrt{J(P \| Q)}$ a true metric [193]. It has the added benefit of not needing the distributions $P, Q$ to be absolutely continuous with respect to each other (i.e., the existence of events $x^{*}$ with $P\left(x^{*}\right)>0$ but $Q\left(x^{*}\right)=0$ is allowed), unlike the (symmetric) Kullback-Leibler divergence, which is undefined in such cases. This makes the Jensen-Shannon divergence an excellent tool even when one has only samples to work with and not complete distributions.

\section{A.2.2.4 Bhattacharyya distance}

The Bhattacharyya distance is defined through the Bhattacharyya coefficient between probability distributions $P(x), Q(x)$

$$
B=\sum_{x} \sqrt{P(x) Q(x)}
$$

The distance is then

$$
D_{B}=-\log B
$$

This "distance" does not obey the triangle inequality but the Hellinger distance

$$
D_{H}=\sqrt{1-B}
$$

does.

\section{A.2.2.5 Kolmogorov-Smirnov and Cramér distances}

Unlike the others, the distance measures described here operate on the (empirical) cumulative distribution functions $F(x), G(x)$. They are induced by the $L_{p}$ norms

$$
D_{p}(F, G)=\|F-G\|_{p}=\left(\sum_{x}|F(x)-G(x)|^{p}\right)^{1 / p}
$$

where the sum is replaced by an integral for continuous distributions. The Cramér distance [194] is derived from the Euclidean norm

$$
D_{2}(F, G)=\left(\sum_{x}|F(x)-G(x)|^{2}\right)^{1 / 2},
$$


and the Kolmogorov-Smirnov distance (see Section A.1.2.2) is derived from the supremum norm

$$
\begin{aligned}
D_{\infty}(F, G) & =\lim _{p \rightarrow \infty}\left(\sum_{x}|F(x)-G(x)|^{p}\right)^{1 / p} \\
& =\sup _{x}|F(x)-G(x)| .
\end{aligned}
$$

\section{A.3 Data and Covariance}

The data we are interested in can be viewed as $n$ observations consisting of $m$ features (e.g., lengths, velocities, etc...). These can be written in terms of a $n \times m$ matrix $\mathbf{y}$ whose rows correspond to the individual observations $\mathbf{v}_{i}$. The simplest statistical measures are then the row-wise mean

$$
\mu_{j}=\frac{1}{n} \sum_{i} y_{i j}
$$

and the unbiased variance

$$
\sigma_{j}^{2}=\frac{1}{n-1} \sum_{i}\left(y_{i j}-\mu_{j}\right)^{2}
$$

where $y_{i j}$ are the matrix elements of $\mathbf{y}$. For many applications it is important to center the data such that the mean vanishes. For this, we introduce the shorthand notation $\mathbf{Y}$ where

$$
Y_{i j}=y_{i j}-\mu_{j}
$$

Additionally, often different features contain data with different dimensions (e.g., mass, length, and velocity of a particle) or are measured on vastly different scales. Many algorithms are not scale invariant. In these cases it can be sensible to rescale the data matrix (thus also non-dimensionalizing it). We define the rescaled data matrix $\tilde{\mathbf{Y}}$ with elements

$$
\tilde{Y}_{i j}=\left(y_{i j}-\mu_{j}\right) / \sigma_{j}
$$

which has zero mean and unit variance. Of course, there are cases in which it is important not to rescale or center, as some information is inevitably lost. In the following, we will make extensive use of the notation introduced above.

Mean and variance reflect variation within each feature. The simplest metric that is able to capture interrelations between different features in the data is the unscaled covariance matrix

$$
\operatorname{Cov}(\mathbf{Y})=\mathbf{Y}^{T} \mathbf{Y}
$$


with elements

$$
\operatorname{Cov}(\mathbf{Y})_{i j}=\sum_{k}\left(y_{i k}-\mu_{k}\right)\left(y_{k j}-\mu_{j}\right)
$$

In order to obtain unbiased estimates for the true statistical covariances, $\operatorname{Cov}(\mathbf{Y})$ needs to be scaled by a factor of $1 /(n-1)$. Then, the off-diagonal elements are the pairwise covariances between features, the diagonal contains the variances. The total variance of the data is given by the trace

$$
\operatorname{Var}(\mathbf{Y})=\operatorname{Tr}(\operatorname{Cov}(\mathbf{Y})) .
$$

If we calculate the covariance of the scaled data, we obtain the correlation matrix $\operatorname{Corr}(\mathbf{y})=\operatorname{Cov}(\tilde{\mathbf{Y}})$. Its off-diagonal elements contain the Pearson correlation coefficients between features. Correlation coefficients lie between -1 and 1 and represent linear relationships between features. Nonlinear dependencies are not detected.

\section{A.4 Covariance analysis}

One of the simplest yet highly informative statistics that can be computed is the covariance matrix (A.30). It contains information about the linear relationships between the different data features. Often, the features are highly correlated and it is interesting to try to disentangle them by finding combinations of features that vary together (e.g., because there is some linear functional relationship between them).

In this section, we describe two simple methods which can make use of the information contained in the covariance matrix to find linear combinations of features that explain a large amount of variance in the data. At the same time, these combinations of features can be used for dimensional reduction, discarding unimportant feature combinations.

\section{A.4.1 Principal Component Analysis}

The covariance matrix is symmetric and real, and therefore admits an eigen-decomposition with real

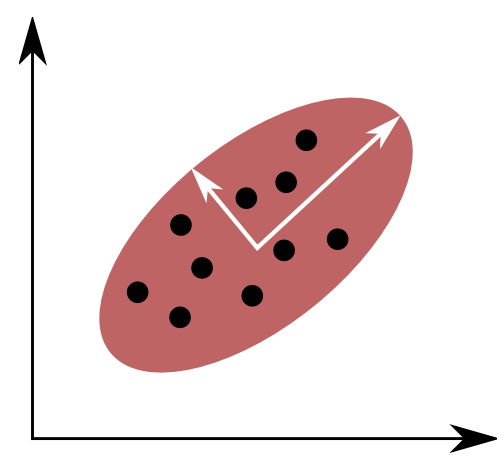

FIGURE A.2: Principal Component Analysis detects orthogonal directions of highest variance (white arrows) in the data. The first principal component (longer arrow) lies in the direction of maximum variance, the second principal component (shorter arrow) is orthogonal to the first. eigenvalues $\lambda_{1} \geq \lambda_{2} \geq \cdots \geq \lambda_{m}$,

$$
\operatorname{Cov}(\mathbf{Y})=\sum_{k} \lambda_{k} \phi_{k} \phi_{k}^{T}
$$


where the set of $\left\{\phi_{k}\right\}_{k}$ form an orthonormal basis of $\mathbb{R}^{m}$. This expression is called Principal Component Analysis (PCA). It was first introduced by Pearson in [195]. Since the total variance is now simply $\operatorname{Var}(\mathbf{Y})=\sum_{k} \lambda_{k}$, the subspaces spanned by the $\phi_{k}$ provide successive orthogonal directions of maximal variance in the data, the first few often containing the most "information" in the sense of total variance (see Figure A.2 for a visual example).

This can be seen as a linear change of variables on the feature space which may have interesting interpretations. The new variables are weighted linear combinations of the features, the weights being given by the eigenvector components $\left(\phi_{k}\right)_{j}$. It can be shown (see [183]) that projecting $\mathbf{Y}$ on the first $k$ such principal components corresponds to an optimal $k$-dimensional approximation of the data in the least squares sense, or equivalently to an optimal fit of the point cloud by a $k$-dimensional ellipsoid.

It should be noted that PCA makes no assumptions whatsoever on the data and solely uses intrinsic information, making it unbiased with regards to any model of the data that might exist. Therefore, PCA is called a non-parametric method.

\section{A.4.2 Factor Analysis}

Closely related to PCA, Factor Analysis (FA) also attempts to find a low dimensional representation of the data [196]. However, it is not entirely intrinsic but makes the assumption that the data can be explained by a linear combination of $k$ latent variables (factors) in conjunction with Gaussian noise. An observation $\mathbf{v}$ (row in $\mathbf{Y}$ ) is written as

$$
\mathbf{v}^{T}=\Gamma \mathbf{z}+\varepsilon .
$$

Here, $\Gamma$ is a $m \times k$ matrix of factor loadings and $\varepsilon \sim \mathcal{N}(0, \Psi)$ is taken from a multivariate Gaussian distribution with zero mean and diagonal covariance matrix $\Psi=\operatorname{diag}\left(\sigma_{1}^{2}, \ldots, \sigma_{m}^{2}\right) .{ }^{1}$ This assumption makes FA a parametric method, in contrast to PCA.

The optimum entries of $\Gamma$ and $\Psi$ for given data can be found using an iterative expectation maximization method [183]. Often, the factor loadings provide similar insights as the PCA components even though they are determined using a different set of assumptions. The covariance matrix as estimated by a factor model is

$$
\Sigma_{\mathrm{FA}}=\Gamma^{T} \Gamma+\Psi,
$$

separating into a term from the factor loadings and Gaussian noise. It is this separation together with a more refined noise model that provides a substantial advantage in comparison with PCA. Whereas PCA simply partitions the

${ }^{1}$ (Probabilistic) PCA can be shown to correspond to a similar model, however with $\Psi=\sigma^{2} \mathbb{I}$. The crucial difference is that in FA, each feature is modeled with independent Gaussian noise. 
variance, FA is able to discard whole features that it explains as noise. Such noise-dominated features can not be detected using PCA. In fact, if the noise variances strongly differ in different features, PCA may wrongly assign a high weight to pure noise.

\section{A.4.2.1 Estimating the number of factors}

The additional assumptions made when using FA allow us to make a probabilistic estimate of the quality of a model with a given number of factors. Given a data set consisting of $n$ i.i.d. observations, the log-likelihood under the assumption of a factor model is [183]

$$
\Lambda_{0}(\mathbf{Y})=-\frac{1}{2} n\left(\log \operatorname{det}\left(2 \pi \Sigma_{\mathrm{FA}}\right)+\operatorname{Tr}\left(\operatorname{Cov}(\mathbf{Y}) \Sigma_{\mathrm{FA}}\right)\right)
$$

We take this assumption as our null hypothesis $H_{0}$. As the alternative hypothesis $H_{A}$ we postulate that the covariance is simply given by its empirical value $\operatorname{Cov}(\mathbf{Y})$. In this case, the log-likelihood is

$$
\Lambda_{A}=-\frac{1}{2} n(\log \operatorname{det}(2 \pi \operatorname{Cov}(\mathbf{Y}))+n) .
$$

We can use the $\chi^{2}$ test to decide whether the factor model must be rejected. The test statistic is $D=-2\left(\Lambda_{0}-\Lambda_{A}\right)$. A factor model with $k$ factors has $k m$ degrees of freedom in the loading matrix $\Gamma$, and $m$ degrees of freedom for the diagonal elements of $\Psi$. The latent variables $\mathbf{z}$ are constrained to be uncorrelated (their correlation matrix is diagonal), and we end up with $d_{0}=k m+m-k(k-1) / 2$ total degrees of freedom. ${ }^{2}$ The alternative matrix is a symmetric matrix, therefore it has $d_{A}=m(m-1) / 2$ degrees of freedom. The log-likelihood ratio $D$ follows a $\chi^{2}$ distribution with $d_{A}-d_{0}$ degrees of freedom, allowing us to calculate a $p$-value. If this $p$-value is less than a pre-set threshold (e.g., 0.05), the factor model must be rejected. Typically, the more factors one chooses, the higher the $p$-value. However, this method of estimating the $p$-value is not without problems when the number of factors in the model exceeds the "true" number of factors [197].

\section{A.5 Clustering algorithms}

Similar to covariance based techniques, clustering methods attempt to uncover structure in a data set by identifying clusters. Data points within one cluster share a high degree of similarity with each other, but are dissimilar to members of different clusters.

In machine learning, clustering algorithms are an example of unsupervised learning, i.e., after choosing some parameter (e.g., the desired number of clusters), ${ }^{2}$ We see that $d_{0}$ can be negative. Such models must be dismissed outright. Thus, there is a constraint on the possible
number of factors that make sense, $d_{0}>0$. 
the algorithm can produce a result directly from the raw data without being trained first.

\section{A.5.1 k-means}

Given a set of $n$ observations $\left\{\mathbf{v}_{i}\right\}$, the goal of the $k$-means algorithm is to find $k$ cluster centroids $\left\{\mathbf{m}_{j}\right\}$ and a partitioning of $\{1, \ldots, n\}$ into $k$ subsets $\left\{S_{j}\right\}$ that minimize the sum of squared distances

$$
\sum_{j=1}^{k} \sum_{i \in S_{j}}\left\|\mathbf{v}_{i}-\mathbf{m}_{j}\right\|^{2} .
$$

Each observation is then assigned to the nearest centroid, forming $k$ clusters in total (see Figure A.3). This is a simple reflection of the rule that intracluster distances should be small (all members of one cluster are close to their centroid).

In general, there are many local minima of the functional (A.38) and finding the globally optimal solution is an NP-hard problem (because it involves the discrete problem of finding an optimum partition of the data points). Given an initial guess for the $\mathbf{m}_{j}$, it can be solved iteratively. However, the quality of the solution strongly depends on the initial guess, and many heuristics to find a good one exist. A possible strategy involves making several guesses for the initial values and then selecting that final converged solution with the smallest value of the functional (A.38).

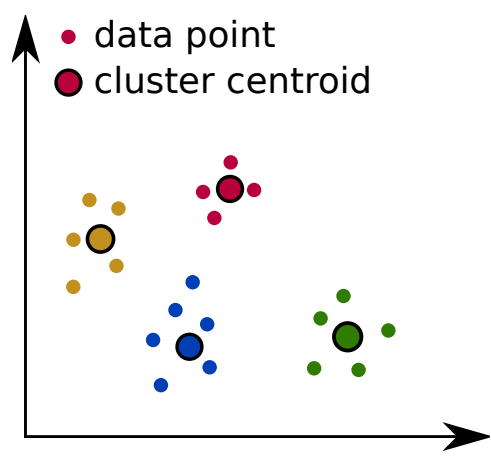

FIGURE A.3: Final configuration of the $k$-means algorithm for $k=$ 4. Cluster centroid positions (large circles) and cluster membership of data points (small circles) are computed such that the functional (A.38) is minimized.

\section{A.5.2 Gaussian Mixing Models}

Instead of partitioning the observations according to distance from a cluster centroid, Gaussian Mixing Models (GMMs) fit a sum of multivariate normal distributions, each corresponding to one cluster. A GMM with $k$ clusters is given by the probability distribution

$$
\begin{aligned}
p\left(\mathbf{v} \mid\left\{\mathbf{m}_{j}, \Sigma_{j}, \lambda_{j}\right\}\right) & =\sum_{j=1}^{k} \frac{1}{\sqrt{\operatorname{det}\left(2 \pi \Sigma_{j}\right)}} \exp \left(-\frac{1}{2}\left(\mathbf{x}-\mathbf{m}_{j}\right)^{T} \Sigma_{j}^{-1}\left(\mathbf{x}-\mathbf{m}_{j}\right)\right) \lambda_{j} \\
& =\sum_{j=1}^{k} \mathcal{N}\left(\mathbf{v} \mid \mathbf{m}_{j}, \Sigma_{j}\right) \lambda_{j}
\end{aligned}
$$




\section{A Statistical Techniques for Phenotyping}

Here, $\Sigma_{i}$ are covariance matrices, $\mathbf{m}_{i}$ are means and $\lambda_{i}$ with $\sum_{i} \lambda_{i}=1$ are weights. The optimum values for these parameters of the model given a set of observations $\left\{\mathbf{v}_{i}\right\}$ can be found using an iterative expectation maximization algorithm (see [183]). As in the case of $k$-means, the algorithm's performance strongly depends on choice of initial values and additionally on constraints placed on the form of the covariance matrices $\Sigma_{i}$ (possible strategies include e.g., constraining $\Sigma_{i}$ to be diagonal or proportional to the unit matrix). Once a set of parameters has been found, an observation $\mathbf{v}$ can be assigned a cluster, e.g., by choosing

$$
C(\mathbf{v})=\underset{j}{\arg \max } \mathcal{N}\left(\mathbf{v} \mid \mathbf{m}_{j}, \Sigma_{j}\right) \lambda_{j}
$$

where $C(\mathbf{v})$ is the cluster index.

\section{A.5.3 Hierarchical clustering}

(a)

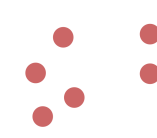

(b)

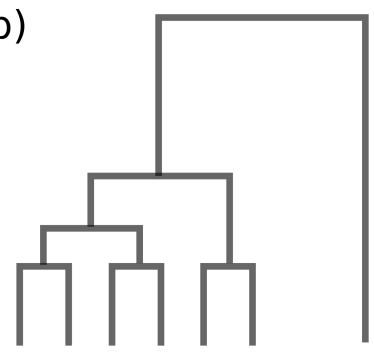

Figure A.4: Hierarchical clustering. (a) Point cloud. (b) Associated dendrogram computed from an agglomerative hierarchical clustering algorithm. The dendrogram reflects distances between points and point clusters.

Instead of specifying the number of clusters, hierarchical clustering algorithms successively combine clusters with minimum distance to new clusters until only one large cluster is left. ${ }^{3}$ The result is a binary tree called a dendrogram that encodes the cluster distance relationships and contains the original data points as leaf nodes (see Figure A.4).

In the case of point clouds the main difficulty lies in determining what a sensible distance between clusters of points is. Popular approaches include centroids between point clouds, the minimum, maximum or average distance between the points belonging to two clusters, as well as Ward's variance based method [144,198].

Some methods (such as Ward's) require an explicit embedding of the data points in an Euclidean space, whereas others (such as the minimum-distance method) only require all pairwise distances between points to be specified.

Hierarchical clustering is very closely related to the hierarchical decomposition algorithm described in Section 4.3.1.

\footnotetext{
${ }^{3}$ This is agglomerative clustering. Divisive clustering exists as well, successively splitting a large cluster into smaller ones.
} 


\section{A.6 Classification algorithms}

Similar to clustering, classification algorithms can reveal structure in unknown data, typically assigning a label to each observation. However, they are an example of supervised learning and need to be trained with data whose class membership is known in advance before they can classify unknown data. Binary (two-class) problems tend to be easier to solve than multi-class problems, but binary classifiers can be converted into multi-class classifiers by calculating class membership probabilities for all pairs of classes (one-vs-one scheme) or by a one-vs-rest scheme.

\section{A.6.1 Linear and Quadratic Discriminant Analysis}

Linear (LDA) and Quadratic Discriminant Analysis (QDA) are amongst the simplest parameter-free multi-class classifiers. First introduced by Fisher [199], they are based on modeling the data in each class by a Gaussian, and then finding an orthonormal projection of the data onto linear subspaces of the feature space such that the between-class variance is maximized while the within-class variance is minimized. If no additional assumptions are made, the decision boundaries (i.e., the loci of points that have equal probability of belonging to either two classes) are quadratic manifolds, and we have QDA. If we add the assumption of homoscedasticity, i.e., the within-class covariance matrices are all equal, the decision boundaries become hyperplanes, and we have LDA. The basic properties of LDA are visualized in Figure A.5.

\section{A.6.1.1 Decision boundaries}

We now derive the equation describing the decision boundary for QDA and LDA. For the sake of simplicity, assume that we are only dealing with a binary classification problem. Each class is modeled by a normal distribution

$$
\begin{aligned}
& p_{1}(\mathbf{v})=\mathcal{N}\left(\mathbf{v} \mid \mathbf{m}_{1}, \Sigma_{1}\right) \\
& p_{2}(\mathbf{v})=\mathcal{N}\left(\mathbf{v} \mid \mathbf{m}_{2}, \Sigma_{2}\right) .
\end{aligned}
$$

The decision boundary is given by the set of points for which $p_{1}(\mathbf{v})=p_{2}(\mathbf{v})$. We take the logarithm of this expression to obtain the equation defining the decision boundary,

$$
\begin{aligned}
(\mathbf{v}- & \left.\mathbf{m}_{1}\right)^{T} \Sigma_{1}^{-1}\left(\mathbf{v}-\mathbf{m}_{1}\right)+\log \operatorname{det} \Sigma_{1} \\
& \quad-\left(\mathbf{v}-\mathbf{m}_{2}\right)^{T} \Sigma_{2}^{-1}\left(\mathbf{v}-\mathbf{m}_{2}\right)-\log \operatorname{det} \Sigma_{2}=0 .
\end{aligned}
$$

Equation (A.42) is quadratic in $\mathbf{v}$, its solution is the decision boundary for QDA. Adding the homoscedasticity assumption $\Sigma_{1}=\Sigma_{2} \equiv \Sigma$, the quadratic terms 
cancel and we obtain the linear decision boundary equation

$$
\left(\mathbf{m}_{2}-\mathbf{m}_{1}\right)^{T} \Sigma^{-1} \mathbf{v}+\mathbf{m}_{1}^{T} \Sigma^{-1} \mathbf{m}_{1}-\mathbf{m}_{2}^{T} \Sigma^{-1} \mathbf{m}_{2}=0,
$$

defining the decision boundary for LDA. We see that LDA is a special case of QDA.

\section{A.6.1.2 Variance maximization}

We now show how to find a projection of the data onto a subspace of the feature space which maximizes between-class variance while minimizing within-class variance. For the sake of simplicity we again restrict ourselves to binary classification. As before, let the data from classes 1,2 be modeled by Gaussians. Let $f_{1,2}$ be the fractions of data points in class 1,2 . We want to find an optimal projection vector $\mathbf{w}$ such that

$$
F(\mathbf{w})=\frac{\mathbf{w}^{T}\left(\mathbf{m}_{1}-\mathbf{m}_{2}\right)\left(\mathbf{m}_{1}-\mathbf{m}_{2}\right)^{T} \mathbf{w}}{\mathbf{w}^{T}\left(f_{1} \Sigma_{1}+f_{2} \Sigma_{2}\right) \mathbf{w}}=\frac{\mathbf{w}^{T} A \mathbf{w}}{\mathbf{w}^{T} B \mathbf{w}}
$$

is maximized. The numerator of this Rayleigh quotient is the projected between-class separation, measured by the squared distance of the projected means. The denominator is the weighted total within-class variance. The optimal projection can be found by differentiating (A.44) with respect to $\mathbf{w}$ and setting the resulting expression to zero. This result can be generalized to multi-class problems. In general, one can find up to $C-1$ projections, where

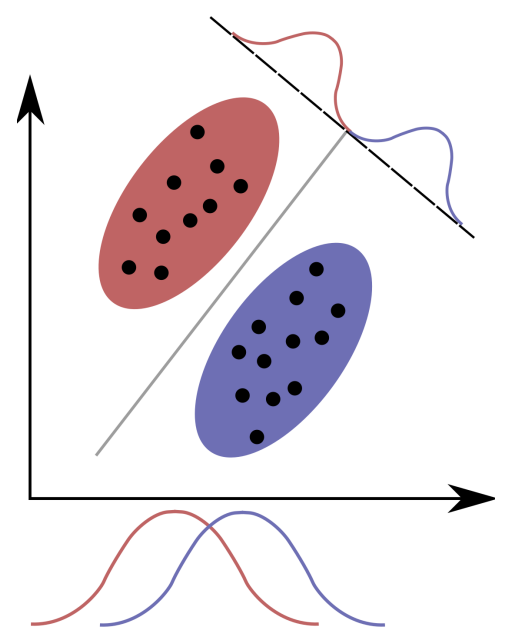

FIGURE A.5: Linear Discriminant Analysis projection and decision boundary. Shown is an example data set consisting of two classes. Projecting on either coordinate axis leads to a difficult to separate distribution. LDA finds a decision boundary (light gray) and an associated projection in which the two classes are optimally separated. $C$ is the number of classes [183].
tion is visualized in Figure A.5.

In order to predict class membership of a new data point, one calculates a score. In the case of binary classification this is simply the signed distance to the separating hyperplane. The sign then tells us on which side of the hyperplane the point falls, and thus how to classify it. This idea can be extended to multi-class predictions as well [200]. 


\section{B The Leaf Data Set}

In this chapter we describe the leaf data set and the vectorization process used to convert leaf scans into weighted graph representations, as well as the preprocessing steps necessary for hierarchical decomposition. The section on vectorization is taken from the supplementary information to [128].

\section{B.1 The data set}

The leaf data set analyzed in Part II consists of 207 vouchered leaves and leaflets collected by Douglas C. Daly, cleared, stained and mounted in resin at the New York Botanical Garden [151]. Most of them belong to the frankincense and myrrh family Burseraceae. They were scanned using a high-resolution film scanner at 6400 dpi by Eleni Katifori. After vectorization by Jana Lasser, we extracted a number of local geometrical quantities for each leaf, as described in Part II. We discarded from the analysis all leaves with fewer than 256 areoles (either very small or badly stained/damaged specimens), leaving us with 186 good quality leaves to analyze.

Among the 186 good quality leaves there are 98 specimens from the genus Protium, 21 from Bursera, 8 from Parkia, and the rest from various other genera. All species' natural habitat is in Southern America.

What follows is a complete list of all specimens.

Protium grandifolium, Protium ovatum, Bursera ovata, Bursera shaferi, Protium dawsonii, Bursera simaruba, Protium aracouchini, Protium altsonii, Bursera gracilipes (2x), Bursera spinescens (3x), Commiphora leptophloeos (3x), Protium calanense, Protium copal, Protium calanense, Bursera ovalifolia, Protium aracouchini, Bursera shaferi, Protium altsonii, Protium leptostachyum, Tetragastris breviacuminata, Crepidospermum atlanticum, Bursera simaruba, Protium brasiliense (2x), Protium ptarianum, Protium rubrum, Protium confusum (2x), Protium gallicum, Protium altsonii, Protium elegans, Protium cuneatum, Protium divaricatum subs. krukofii, Protium glaucescens, Protium guianense, Protium giganteum, Protium attenuatum, Protium trifoliolatum, Protium cubense, Protium apiculatum, Protium camosum, Protium carolense, Protium connarifolium, Dacryodes belemensis, Bursera attenuate, Tetragastris occhionii, Pouteria filipe, Pouteria torta, Pouteria glomerata, Bowdichia nitida, Andira macrothrysa, Apuleia leiocarpa, Diploon cuspidatum, Brosimum guianensis, Hymenaeae parvifolia (2x), Schizolobium amazonicum (2x), Ocotea sp. 1, Apuleia leiocarpa (2x), Dipteryx ferrea, Cedrela odorata, Pouteria coriaceae, Clarisia racemosa, Eschweilera sp., Licania cuspidata, Protium heptaphyllum, Astronium lecointei, Dalbergia miscolobium, Terminalia oblonga (2x), Shefflera morototoni, Dipteryx alata, Dipteryx ferrea, Aspidospenna parvifolium, Diallium guianensis, Batocarpus amazonicus, Walsura sp. 2, Jacaranda copaia, Dalbergia miscolobium, Qualea grandiflora, Parkia nitida (8x), Cedrela odorata, Brosimum guianensis, Ocotea sp. 2, Tabebuia serratifolia, Schizolobium amazonicum, Unknown species 2, Enterolobium sp. 1, Unknown species 3, Nectandra cuspidata, Apuleia leiocarpa, Enterolobium sp. 3, Protium sp. nov. 8, Protium pittieri, Tetragastris hostmanii, 
Protium pallidum, Protium tenuifolium, Protium sp. nov. 3 aff. P. warmingianum, Protium neglectum, Bursera hollikii (2x), Protium madagascariense (2x), Protium subserratum, Protium nitidifolium, Protium nervosum, Canarium planifolium, Bursera aromatica, Protium polybotryum, Protium glabrurm, Protium inodorum, Protium rynchophyllum, Protium tovarense, Protium llanorum, Protium mcleodii, Protium crenatum, Protium multiramiflorum, Protium sp. nov. 7, Protium sp. nov. aff. P. montanum, Protium sp. nov. 10, Protium subserratum, Bursera inversa, Protium strumosum, Protium sagotianum, Protium occultum, Protium demerarense, Protium sp. nov. 9, Protium subserratum "lobo", Protium reticulatum, Protium ravenii, Bursera aromatica, Protium divaricatum, Protium gallosum, Protium krukovii, Protium sp. nov. 6, Protium sp. nov. 4, Protium sp. nov. 11, Protium laxiflorum, Protium hebetatum, Canarium obtusifolium (2x), Protium decandrum, Canarium nitidifolium, Protium sp. nov. 13, Protium morii, Protium urophyllidium, Protium sp. nov. 5, Protium grandifolium, Protium puncticulatum, Protium glabrescens, Canarium scholasticum, Protium divaricatum, Protium heptaphyllum, Protium sp. nov. 13, Protium pittieri, Protium copal, Protium subacuminatum (2x), Protium guianense, Protium icicariba, Protium sprumanum, Protium unifolioiatum, Protium serratum, Protium heptaphyllum, Protium beandou, Protium opacum subs. opacum, Protium sp. nov. 12 (2x), Bursera glabrifolia (4x),

The full leaf venation feature data set can be found in the online supplement to [128].

\section{B.2 Vectorization}

The extraction the networks from the original high-resolution scans can be divided into two main steps: segmentation of the image to create a suitable binary representation and skeletonization of the shapes.

To segment the image we use a combination of Gaussian blurring to reduce noise, local histogram equalization and recombination with the original image to increase contrast, and Otsu thresholding [201] to find the optimal threshold for the creation of the binary image.

For the skeletonization we use a vectorization technique known from optical sign recognition [202, 203]. The approach relies on the extraction and approximation of the foreground features' contours using the Teh-Chin dominant point detection algorithm [204] and subsequent triangulation of the contours via constrained Delaunay triangulation [205]. The foreground is partitioned into triangles that are used to create a skeleton of the shape. Each triangle contributes a central point to the skeleton that is determined by searching for local maxima in the Euclidean distance map [206] of the binary. Together, these central points approximate the skeleton.

By looking at edges shared between two triangles, neighborhood relations can be established and an adjacency matrix can be created. This adjacency matrix defines a graph composed of nodes (the former triangle centers) and edges (the connections between two adjacent triangles). In addition to the topology of the graph the original geometry of the network including coordinates of the nodes and lengths and radii of edges are preserved and stored in the graph.

The processing is done using algorithms implemented in the Python programming language. The package uniting this functionality was written by Jana 
Lasser. It is called NET, and is available at

http://github.com/JanaLasser/network_extraction/.

Earlier digitization work [153] used other techniques and can be limited in terms of resolution [155, 207]. Our vectorization process is visualized in Figure B.1.
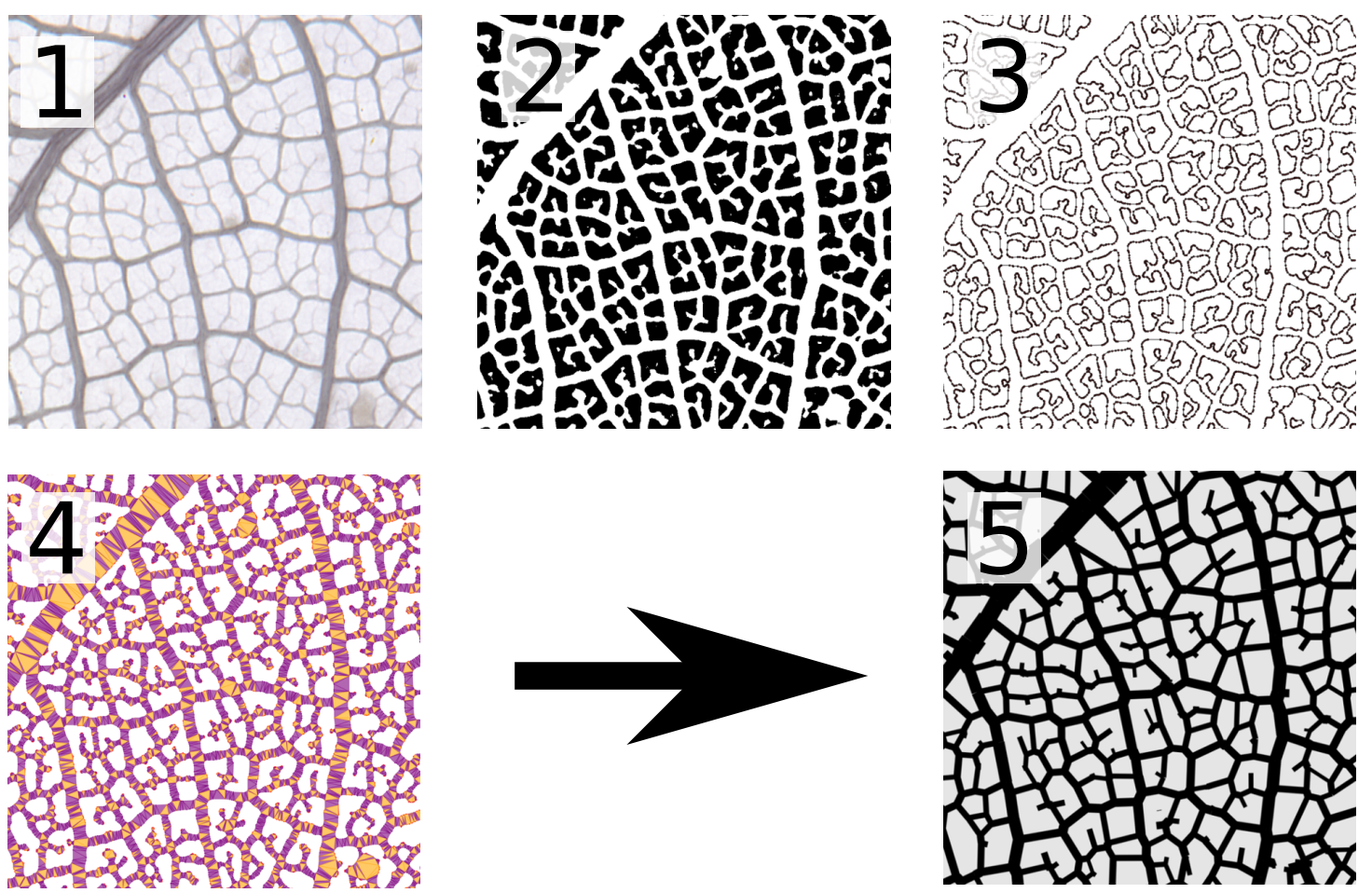

FIGURE B.1: The vectorization process. (1) We start from a high resolution scanned image (6400 dpi) of the leaf. (2) A binary is generated using a combination of blurring, local histogram equalization and finally Otsu thresholding. (3) Teh-Chin dominant point detection is used to obtain a set of contour points. (4) Constrained Delaunay triangulation of the contour points. (5) The final graph representation of the vascular network. Although we are showing a small crop, the process was performed for a whole leaf.

\section{B.3 Automatic pre-processing of the leaf graphs}

Because the vectorization procedure may produce errors in the leaf network graphs, several pre-processing steps are applied to minimize their impact and prepare the networks for hierarchical decomposition. The first step of preprocessing is visual inspection of a plot of the graph and manual removal of obvious artifacts, such as erroneously vectorized dirt and smudges. Next, a set of automatic procedures is applied to remove spurious crossing edges and spurious (almost) collinear edges. Finally, the graph is pruned and the loopy backbone constructed. From this, the fundamental cycle basis is computed. 


\section{B.3.1 Removal of nonplanar artifacts}

It is possible that during vectorization, crossings of some edges appear. This can be due to numerical errors or errors during creation of the graph skeleton. Such artifacts are removed by a heuristic method, iterating over all nodes $n$ of the network and considering their 5-neighborhoods (i.e., the subgraph of all nodes which are at most 5 hops away from $n)$. This is an arbitrary choice which works sufficiently well in practice. ${ }^{1}$ For all pairs of edges in the 5-neighborhood, it is tested whether they intersect, and if they do, both edges are removed from the graph. Typically not more than one or two crossings are detected in each whole leaf network, most networks contain none at all.

\section{B.3.2 Removal of collinear edges}

It is possible that during vectorization, spurious cycles occur in which the edges $(a b),(b c),(a c)$ exist, but $(a b)$ and $(a c)$ are exactly or very nearly collinear. Such edges are removed by testing all edges ending in a certain node for collinearity by calculating the

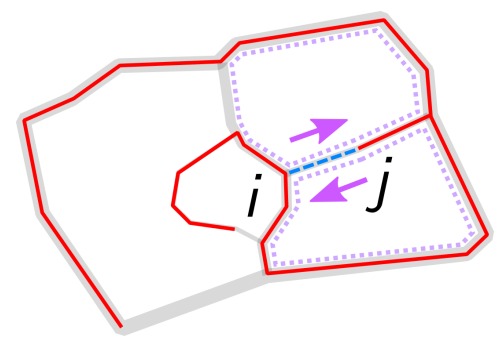

FIGURE B.2: Detecting fundamental cycles (facets) in a planar graph without treelike components. First, a minimum spanning tree $M$ is constructed (red). Then, each edge $(i j) \notin M$ is taken as the starting point of two cyclic traversals, using the embedding to consistently take the left-most (or right-most) direction at every junction. This way, each traversal is guaranteed to end up at the starting edge again having traversed exactly the edges of one facet.

inner product of their normalized associated vectors,

$$
\cos \phi=\overrightarrow{(a b)} \cdot \overrightarrow{(a c)} \text {. }
$$

If collinear edges are found (up to a threshold of $\cos \phi<10^{-3}$ ), the longer one is removed. Typically, up to ten such edge-pairs can occur in a leaf network graph.

\section{B.3.3 Cycle pruning}

Hierarchical decomposition works only on the "loopy" part of a network that makes up the associated planar polygon. Therefore, all edges that are not part of a (topological) cycle are removed. This is done by first computing an arbitrary cycle basis using [208] (which does not necessarily correspond to the basis given by the facets of the polygon but can be computed quickly). Then, all edges of the graph that are not part of at least one cycle in the basis are removed. This is equivalent to removing all "treelike components" of the network (see Figure 4.1). Biologically, the smallest treelike components are crucial for uniform water delivery [209], and in a future version of the method, we intend to include them in some way.

\footnotetext{
${ }^{1}$ To be guaranteed to always work, one would have to search the whole graph instead of a small neighborhood.
} 


\section{B.3.4 Choice of connected component}

If there are several connected components in the network after cycle pruning, we choose the largest one to do hierarchical decomposition. This is done in order to remove artifacts stemming from vectorizing dirt or smudges in the original image, or from small freely ending veinlets which appear disconnected because image resolution was not high enough for the vectorizer to reliably detect a connection to the rest of the network.

\section{B.4 Constructing the fundamental cycle basis.}

The fundamental cycles of a graph are given by the facets of the loopy backbone graph $G$ seen as a planar polygon. A simple way of constructing such a basis is to first construct a minimum spanning tree $M$. Then, starting at each edge $(i j) \notin M$ and the reverse edge $(j i)$, traverse the graph in such a way that at each node $n_{i}$, the next node is chosen to be the left-most (or right-most) as seen from the unit vector $\mathbf{n}_{i-1, i}$ that points from node $i-1$ to node $i$. Let the current edge be $(i j)$, and a decision needs to be made about where to proceed. To do this, first the unit vectors $\mathbf{n}_{i j}$ (pointing from point $n_{i}$ to point $n_{j}$ ) and $\left\{\mathbf{n}_{j k}\right\}_{k}$ for all neighbors $k \neq i$ of $j$ are formed. Then, we calculate the set of $\left\{S_{k}\right\}_{k}$ with $S_{k}=\operatorname{sign}\left(\sin \alpha_{k}\right)\left(1-\cos \alpha_{k}\right)$, where $\alpha_{k}$ is the angle between $n_{i j}$ and $n_{j k}$, chosen on the interval $[-\pi, \pi]$ (see Figure B.3). The maximum value of $S_{k}$ is attained for the left-most direction as seen from $n_{j}$, coming from $n_{i}$. The corresponding node $k^{\star}$ will be visited next, and the next edge is $\left(j k^{\star}\right)$. This way, one can never leave

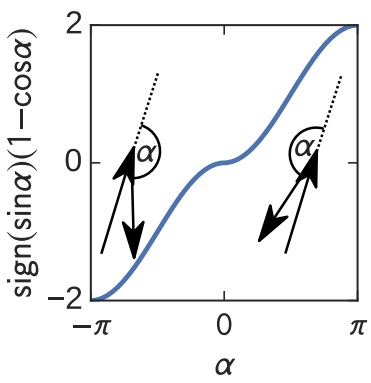

FIGURE B.3: If $\alpha$ is the angle between two vectors, the function $S=\operatorname{sign}(\sin \alpha)(1-\cos \alpha)$ allows one to determine how far the second vector is to the left or right of the first. the cycle corresponding to one facet. The procedure is shown in Figure B.2. Traversal is complete once the same node is encountered that one had started with. 



\section{Nesting Number Analytics}

(a)

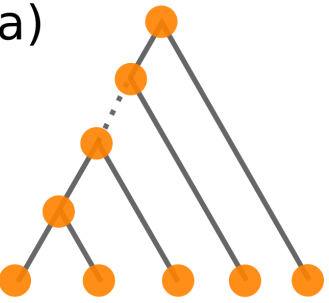

(b)

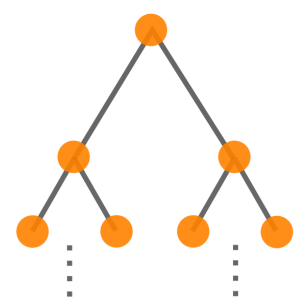

(c)

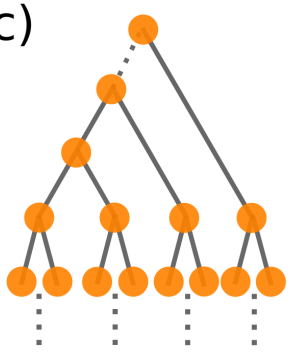

(d)

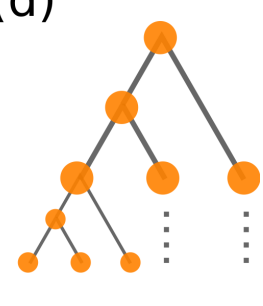

FIGURE C.1: Nesting tree model topologies. (a) Perfectly additive tree. (b) Perfectly multiplicative tree. This topology is also called a fully balanced binary tree. (c) Mixed tree. Here, $n$ perfect multiplicative trees of given size are attached to the leaf nodes of a perfectly additive tree. (d) Iteratively self-similar tree. At each iteration step $k$, copies of the initial tree $T_{0}$ are attached by their roots to the leaf nodes of the tree $T_{k-1}$.

In this chapter, we provide some analytical results for the nesting ratios and nesting numbers of several model topologies. Some of these were published before in [58]. The model topologies we consider are perfectly additive, perfectly multiplicative, mixed, and iteratively self-similar (see Figure C.1). For each type, we calculate the nesting ratios $q_{j}$, the unweighted nesting number $Q_{u}$ and the degree weighted nesting number $Q_{w}$.

(a) Perfectly additive tree. Let the degree of the whole tree be $d$. We label the bifurcating vertices starting with the lowest one. Then it is easy to see that the nesting ratios are

$$
q_{j}=\frac{1}{j}, \quad j=1,2, \ldots, d-1
$$

Similarly, the weights are

$$
\begin{aligned}
w_{j} & =j \\
w & =\sum_{j=1}^{d-1} w_{j}=\frac{1}{2} d(d-1) .
\end{aligned}
$$

Calculating the weighted nesting number, we end up with

$$
Q_{w}=\frac{2}{d(d-1)} \sum_{j=1}^{d-1} j \frac{1}{j}=\frac{2}{d} .
$$


Similarly, the unweighted nesting number is

$$
Q_{u}=\frac{H_{d-1}}{d-1}
$$

where we introduced the harmonic numbers $H_{n}=\sum_{i=1}^{n} i^{-1}$. In the limit $n \rightarrow \infty$, both nesting numbers vanish. ${ }^{1}$

(b) Perfectly multiplicative tree. The nesting ratios are all equal to 1, therefore

$$
Q_{u}=Q_{w}=1 \text {. }
$$

(c) Mixed tree. This tree model consists of $k$ perfectly multiplicative trees with $2^{\ell}$ leaf nodes each chained together by an additive tree. It is supposed to serve as a model for real leaf network hierarchies which consist of many approximately multiplicative elements at the smallest level, chained together in additive fashion at the largest level. First, we calculate the nesting ratios, noting that they are vanishing for all nodes in the perfectly multiplicative subtrees. Enumerating the nodes of the additive tree as in (a), their nesting ratios are

$$
\begin{aligned}
& q_{1}=1 \\
& q_{j}=\frac{2^{\ell}}{2^{\ell} j}=\frac{1}{j}, \quad 2 \leq j \leq k-1 .
\end{aligned}
$$

In all $k$ perfectly multiplicative subtrees, there are $2^{\ell-1}$ nodes with weight $2-1,2^{\ell-2}$ nodes with weight $2^{2}-1$, and so on. The additive nodes have weights $w_{j}=(j+1) 2^{\ell}-1$. Thus we can calculate the total weight

$$
\begin{aligned}
w_{k, \ell} & =k \sum_{s=1}^{\ell} 2^{\ell-s}\left(2^{s}-1\right)+\sum_{j=1}^{k-1}\left((j+1) 2^{\ell}-1\right) \\
& =2^{\ell-1}(k(2 \ell+k-1)-2)+1
\end{aligned}
$$

The weighted nesting number for a $k, \ell$ mixed tree is therefore

$$
\begin{aligned}
Q_{w}^{(k, \ell)} & =1-\frac{1}{w_{k, \ell}} \sum_{j=2}^{k-1} \frac{1-j}{j}\left((j+1) 2^{\ell}-1\right) \\
& =1-\frac{1}{w_{k, \ell}}\left(2^{\ell}(k \ell-1)+k+\left(2^{\ell}-1\right) H_{k-1}\right) .
\end{aligned}
$$

Here, we used the fact that $Q_{w, u}=1-R_{w, u}$, where in $R_{u, w}$ all nesting

\footnotetext{
${ }^{1}$ Asymptotically, the harmonic numbers behave as $H_{n} \sim \log n+\gamma$, where $\gamma$ is the Euler-Mascheroni constant.
} 
ratios are replaced by the subtree asymmetries $a_{j}=1-q_{j}$. This way, all contributions from the perfectly multiplicative trees vanish. As a test, we calculate $Q_{w}^{(k, 0)}=\frac{2}{k}$, reproducing the nesting number of the perfectly additive model. Performing all the summations with trivial weights we find for the unweighted nesting number

$$
Q_{u}^{(k, \ell)}=\frac{H_{k-1}+k\left(2^{\ell}-1\right)}{k-1+k\left(2^{\ell}-1\right)}
$$

again reproducing the correct nesting number in the case when $\ell=0$.

(d) Iteratively self-similar architecture. This tree is constructed through an iterative procedure starting from any given initial tree with $n$ leaf nodes by successively attaching copies of itself to the leaf nodes. It is easy to see that, defining the initial tree as $k=1$, at step $k$ the tree has $n^{k}$ leaf nodes and, defining the initial tree as level $\ell=0$, there are $t_{k, \ell}=n^{\ell}$ copies of the initial tree at level $0 \leq \ell<k$. Labeling each bifurcating node of the initial tree by an index $i=1, \ldots, n-1$, we can label each node by a triple

$$
(\ell, m, i), \quad 0 \leq \ell<k, \quad 1 \leq m \leq t_{k, \ell}, \quad 1 \leq i \leq n-1 .
$$

Here, $m$ counts the tree copies at level $\ell$. A node in a copy at level $\ell$ that had degree $d_{i}$ in the original tree has degree $d_{i, k}=d_{i} n^{k-\ell-1}$. In order to calculate all nesting ratios, we note that if one node had nesting ratio

$$
q_{i}=\frac{s_{i}}{r_{i}}, \quad r_{i} \geq s_{i}
$$

in the initial tree, then at step $\mathrm{k}$ it will have partition ratio

$$
q_{k, \ell=0, m, i}=\frac{s_{i} n^{k-1}}{r_{i} n^{k-1}}=q_{i}
$$

Since any subtree copy is really just a copy of the whole but at an earlier iteration step, we generally find $q_{k, \ell, m, i}=q_{i}$. With the above information, we easily see that the weights are

$$
w_{k, \ell, m, i}=d_{i} n^{k-\ell-1}-1 .
$$

Leading us to the total weight

$$
\begin{aligned}
w_{k} & =\sum_{\ell=0}^{k-1} \sum_{m=1}^{n^{\ell}} \sum_{i=1}^{n-1}\left(d_{i} n^{k-\ell-1}-1\right) \\
& =k n^{k-1}\left(w_{1}-1\right)+(k-1) n^{k}+1 .
\end{aligned}
$$


The weighted nesting number is now

$$
\begin{aligned}
Q_{w}^{(k)} & =\frac{1}{w_{k}} \sum_{\ell=0}^{k-1} \sum_{m=1}^{n^{\ell}} \sum_{i=1}^{n-1} w_{k, \ell, m, i} q_{i} \\
& =\frac{k n^{k-1} Q_{w}^{(1)} w_{1}+\left((k-1) n^{k}-k n^{k-1}+1\right) Q_{u}^{(1)}}{k n^{k-1}\left(w_{1}-1\right)+(k-1) n^{k}+1},
\end{aligned}
$$

where we introduced the unweighted nesting number of the initial tree

$$
Q_{u}^{(1)}=\frac{1}{n-1} \sum_{i=1}^{n-1} q_{i}
$$

Thus we were able to reduce the weighted nesting number of any iteratively self-similar tree at iteration step $k$ to an expression of the nesting numbers of the initial tree. Another form of (C.15) is

$$
Q_{w}^{(k)}=Q_{w}^{(1)}+\frac{Q_{u}^{(1)}-Q_{w}^{(1)}}{1+\frac{w_{1}}{\frac{n}{k}\left(k-1+n^{-k}\right)-1}} .
$$

It is instructive to calculate the limit for infinite trees,

$$
\begin{aligned}
Q_{w}^{(\infty)} & =\lim _{k \rightarrow \infty} Q_{w}^{(k)} \\
& =Q_{w}^{(1)}+\frac{Q_{u}^{(1)}-Q_{w}^{(1)}}{1+\frac{w_{1}}{n-1}} .
\end{aligned}
$$

Whether the nesting number of the self-similar tree decreases or increases with the iteration step depends on the difference $Q_{u}^{(1)}-Q_{w}^{(1)}$. As an example, consider the case of a self-similar tree composed of perfectly additive trees of degree $n$. Then

$$
Q_{u}^{(1)}-Q_{w}^{(1)}=\frac{H_{n-1}}{n-1}-\frac{2}{n}
$$

which is positive for all $n$.

The unweighted nesting index at any iteration step $k$ is simply $Q_{u}^{(k)}=Q_{u}^{(1)}$ because of equation (C.12). 


\title{
D Linear Flow Networks
}

\author{
Everything flows.
}

(Heraclitus of Ephesus, attributed)

In this section we introduce the basic mathematical framework needed to describe flow networks. Flow networks can be used to model optimal venation networks, vein morphogenesis, power grids, and much more.

We sketch a unified theory of linear flow networks that includes the effects of edge weight perturbations. Our theory is based on the idea of the description of flow in terms of either potentials (or pressures) that are defined at the network nodes, or equivalently in terms of cycle flows along the topological cycles of the network. These two descriptions are dual to each other in the sense that they both give the same resulting physical edge flows. The cycle flow description also allows us to look into edge perturbations of the network in the continuum limit in a simple way. We find that they behave similarly to dipoles in electrostatics. This decay behavior is also found in real leaf networks. ${ }^{1}$

We note that some of the ideas in this chapter are new (to the best of our knowledge), in particular the duality of perturbation flows in terms of projection matrices. ${ }^{2}$ This allows us to construct a continuum theory for planar networks and derive a scaling relation for the strength of the perturbation flow as a function of distance from the perturbation. We will use this to compare to simulations in real leaf networks in a later chapter. We note that the duality can be used to speed up calculations, which is relevant for many applications (e.g., in power grids [170]). Unfortunately, these speedup results do not fit well with the rest of this thesis. They are available in [160].

For the basic graph theory, we follow the excellent textbook [210]. We make limited use of concepts from algebraic topology. A very comprehensive resource about this topic is [211]. Some earlier ideas about cycle and cocycle spaces can be found in [212].

\section{D.1 Linear algebra of graphs}

In this section we define the basic concept of a graph and the associated linear algebraic concepts that allow us to model flow networks. We start with the most abstract definition.

\footnotetext{
${ }^{1}$ Unpublished observation in our vectorized leaf graphs.

${ }^{2}$ The fact that equation (D.15) is true has been well-known for a long time. The original idea to decompose a perturbation flow into cycle flows is due to Dirk Witthaut. What is new to the best of our knowledge is the connection between equations (D.24) and (D.25).
} 
A graph $G=(V, E)$ consists of a set of nodes $V=\left\{v_{1}, \cdots v_{N}\right\}$ and a set of edges $E=\left\{e_{1}, \cdots e_{L}\right\}$ where each edge $e \in V \times V$ "connects" two nodes. A subgraph of $G$ is defined by subsets $V^{\prime} \subset V$ and $E^{\prime} \subset E$ such that $G^{\prime}=\left(V^{\prime}, E^{\prime}\right)$ is again a graph. An induced subgraph $G\left[V^{\prime}\right]=G^{\prime}$ of $G$ is defined by a node set $V^{\prime} \subset V$ and its edge set is $E^{\prime}=\left\{\{e, f\} \in E \mid e, f \in V^{\prime}\right\}$. Let $N_{G}(v)=$ $\{w \in V \mid \exists x \exists\{w, x\} \in E\}$ be the set of neighbors of $v$ in $G$. Then we call $d_{G}(v)=\left|N_{G}(v)\right|$ the degree of node $v .{ }^{3}$ We define the line graph $L(G)=(E, F)$ with node set $E$ and edge set $F$ such that two nodes in $L(G)$ are adjacent if and only if the associated edges in $G$ share one node. A path is a nonempty graph with $V=\left\{v_{1}, \cdots, v_{k}\right\}, E=\left\{\left\{v_{1}, v_{2}\right\},\left\{v_{2}, v_{3}\right\}, \cdots,\left\{v_{k-1}, v_{k}\right\}\right\}$ where the $v_{i}$ are pairwise disjoint. A cycle is a path for which $v_{1}=v_{k}$. A graph is called connected if there exists a path between any two of its nodes.

A graph is called planar if there exists an embedding map $\varphi: V \rightarrow \mathbb{R}^{2}$ such that, if lines are drawn on the plane between all adjacent nodes, no two lines cross. The embedding map is generally not unique.

Next, we define and analyze the three fundamental vector spaces that can be associated to a graph. They correspond to the nodes, oriented edges, and oriented cycles of G (see Figure D.1 (a)).

(a)

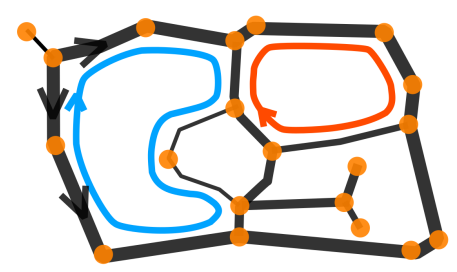

(b)

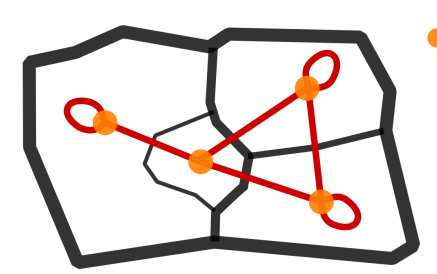

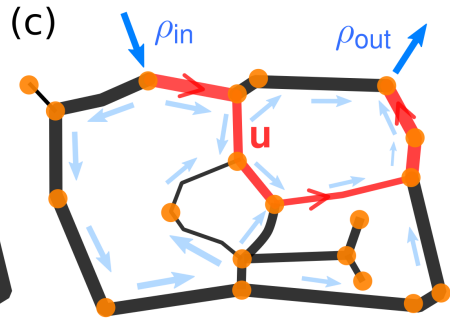

FIGURE D.1: Illustration of oriented graphs (this one is planar) and flows. (a) Nodes (orange dots); oriented edges (gray lines between dots), only some orientations have been indicated; oriented cycles (blue and red), orientation has been indicated. Note that cycle orientation and edge orientation need not coincide. (b) The algebraic dual graph constructed from the graph in (a) (red edges). (c) A flow (light blue arrows) with one source $\rho_{\text {in }}$ and one sink $\rho_{\text {out }}$ (dark blue arrows). One particular choice of $\mathbf{u}$ for this flow is shown in red. The physical flow is a superposition of this particular flow and cycle flows.

\section{D.1.1 The node vector space}

The neighborhood relationships between nodes are encoded in the $|V| \times|V|$ adjacency matrix $A$, with entries

$$
A_{i j}= \begin{cases}1 & \left\{v_{i}, v_{j}\right\} \in E \\ 0 & \text { otherwise }\end{cases}
$$

\footnotetext{
${ }^{3}$ If the context is clear, we drop the subscript $G$ from the notation.
} 
The adjacency matrix maps the node space $A: \mathcal{V} \rightarrow \mathcal{V}$, where $\mathcal{V} \simeq \mathbb{R}^{|V|}$. In the standard basis of $\mathcal{V}$ (where the $i$ th basis vector has a 1 at the $i$ th position and zeros otherwise), the $i$ th basis vector is associated to the respective node $v_{i}$.

A weighted graph is $G=(V, E, K)$, where $K: E \rightarrow \mathbb{R}$ is the weight function. ${ }^{4}$ The weighted adjacency matrix has entries

$$
K_{i j}= \begin{cases}K\left(\left\{v_{i}, v_{j}\right\}\right) & \left\{v_{i}, v_{j}\right\} \in E \\ 0 & \text { otherwise }\end{cases}
$$

Clearly, any graph is completely defined by its adjacency matrix or by its weighted adjacency matrix if all weights are non-zero. A related matrix is given by the (weighted) Laplacian

$$
L=D-A,
$$

where the diagonal matrix with entries $D_{i i}=\sum_{j} A_{i j}$ is the degree matrix of $G$. The (weighted) Laplacian also defines $G$ completely in a similar fashion.

\section{D.1.2 The edge vector space}

More linear structure can be defined by equipping $G$ with an orientation (arbitrary but fixed) of the edges. This allows us to define the edge vector space $\mathcal{E} \simeq \mathbb{R}^{|E|}$ on which the edge-node incidence matrix $E: \mathcal{E} \rightarrow \mathcal{V}$ operates. It is defined by

$$
E_{v, e}= \begin{cases}+1 & v \text { is the head of edge } e \\ -1 & v \text { is the tail of edge } e \\ 0 & \text { otherwise. }\end{cases}
$$

The Laplacian matrix has a decomposition as $L=E E^{T}$. Defining the diagonal edge weight matrix $K: \mathcal{E} \rightarrow \mathcal{E}$ by $K_{i i}=K\left(e_{i}\right)$, the weighted Laplacian is $L=E K E^{T}$.

\section{D.1.3 The cycle vector space}

The null space $\mathcal{C} \simeq$ ker $E$ is called the cycle space of $G$. This is true because any linear combination of vectors representing the edges of an oriented cycle contain each node twice, once as the head and once as the tail of a particular oriented edge. Therefore, they are mapped to zero by $E$. The cycle space thus contains all linear combinations of oriented edges that form a cycle in $G$. We now construct a basis for the cycle space. Without loss of generality, let $G$ be connected. Let $T$ be a minimum spanning tree of $G$. Then $T$ contains exactly $N-1$ edges. For

\footnotetext{
${ }^{4}$ More generally, the range of $K$ can be any set and not just $\mathbb{R}$.
} 
each $e \notin T$, the (unique) oriented path in $T$ from one end-node of $e$ to the other, together with $e$ itself forms a cycle. The corresponding $|E|-|V|+1$ vectors are linearly independent because each of them contains at least one edge (non-zero component) not present in the others. They form a basis of the cycle vector space [210] (see also Figure D.1 (b)).

Let $\left\{c_{1}, \cdots, c_{|E|-|V|+1}\right\}$ be a basis of the cycle space. We define the cycle-edge incidence matrix $C: \mathcal{C} \rightarrow \mathcal{E}$ with entries

$$
C_{e, c}= \begin{cases}+1 & e \in c \text { with positive orientation } \\ -1 & e \in c \text { with negative orientation } \\ 0 & \text { otherwise }\end{cases}
$$

Using this, we define the dual Laplacian $L^{*}=C^{T} K^{-1} C$, where $K^{-1}$ is the inverse edge weight matrix. If the graph is planar and the cycle basis corresponds to the facets of the graph as seen as a planar polygon, $L^{*}$ is the reduced Laplacian of the topological dual graph $G^{*}$, where the line and column corresponding to the outside boundary cycle have been removed.

In general, $L^{*}$ defines the algebraic dual graph with respect to the given cycle basis. In this graph, each basis cycle corresponds to one node, two nodes are connected if their cycles $c_{1}, c_{2}$ share at least one edge, and the weights are $\sum_{e \in c_{1} \cap c_{2}} K_{e}^{-1}$. This definition of edge weights is natural for linear flow networks (see later). The dual Laplacian $L^{*}$ is always invertible because unlike $E$, the matrix $C$ has full rank by construction.

\section{D.1.4 Algebraic topology of graphs}

Consider the three fundamental vector spaces we can associate to a graph together with the homomorphisms that map between them,

$$
0 \rightarrow \mathcal{C} \stackrel{\mathcal{C}}{\rightarrow} \mathcal{E} \stackrel{\tilde{E}}{\rightarrow} \mathcal{V}^{\perp} \rightarrow 0
$$

Here, we define $\mathcal{V}^{\perp}=\left\{\mathbf{v} \in \mathcal{V} \mid\left\langle\mathbf{v},(1,1, \cdots, 1)^{T}\right\rangle=0\right\}$. A basis of this space is given by the vectors $\mathbf{b}_{i}$ with components

$$
\left(b_{i}\right)_{j}=\delta_{1 j}-\delta_{i+1, j}, \quad i \in\{1, \cdots,|V|-1\} .
$$

This definition makes $\tilde{E}: \mathcal{E} \rightarrow \mathcal{V}^{\perp}$, where $\tilde{E}$ equals $E$ expressed in this basis, an epimorphism (it now has full row rank in this basis of $\mathcal{V}^{\perp}$ ). Physically, this means we fix the gauge by measuring all potentials with respect to node 1 . Because $C$ is a monomorphism (it has full column-rank) and

$$
\tilde{E} C=0,
$$


(D.6) is a short-exact sequence. It is also split because we are dealing with vector spaces [211]. A basic result for short-exact sequences [211] is

$$
\begin{aligned}
\mathcal{V}^{\perp} & \simeq \mathcal{E} / \operatorname{im}(C) \\
& \simeq \mathcal{E} / \mathcal{C} \simeq \mathcal{C}^{*}
\end{aligned}
$$

where $\mathcal{C}=\operatorname{im}(C)$ is called the cycle space and $\mathcal{C}^{*}=\mathcal{C}^{\perp}$ is called the cocycle space [210, 212]. Cocycles, or cuts [210], are those sets of edges of $G$ whose removal cuts $G$ into two disconnected parts. There is a direct correspondence between cuts $\mathcal{C}^{*}$ and physical gauge fixed potentials $\mathcal{V}^{\perp}$ which is given by the isomorphism $\left.\tilde{E}\right|_{\mathcal{C}^{*}}: \mathcal{C}^{*} \rightarrow \mathcal{V}^{\perp}$.

\section{D.2 Linear flow networks}

In this section, we present a unified theory of linear flow networks using the mathematical language from the previous chapter. Linear flow networks can be used to model a wide variety of ubiquitous natural and human-made systems. Applications range from electrical networks and power grids [171, 213] to fluid flow in plant vasculature [72], macroscopic pipe systems [214, 215] or microfluidics [216, 217]. The description is fundamentally steady-state.

Given a graph with weighted adjacency matrix $K_{i j}$, the flow $F_{i j}$ from node $i$ to node $j$ is

$$
F_{i j}=K_{i j}\left(\phi_{j}-\phi_{i}\right)
$$

or more compactly

$$
\mathbf{F}=K E^{T} \phi,
$$

where $\mathbf{F} \in \mathcal{E}$ is the vector of flows and $\phi \in \mathcal{V}$ is the vector of potentials. $\mathbf{F}$ may represent volume flows of water or gas in plants or pipe networks, or flow of electrical power in power grids. In the respective cases, $\phi$ represents hydrostatic pressure or voltage angles. In the case of power grids, the linear equation (D.11) is an approximation to a more general non-linear law [170]. In order to be physical, the flow vector needs to satisfy the boundary conditions (Kirchhoff's laws)

$$
\begin{aligned}
E \mathbf{F} & =\rho \\
C^{T} K^{-1} \mathbf{F} & =0 .
\end{aligned}
$$

Equation (D.12) is the junction rule; the sum of all incoming flows at a given node $i$ must equal the net flow $\rho_{i}{ }^{5}$ Equation (D.13) is the mesh rule; the sum of

\footnotetext{
${ }^{5}$ Note that for convenience, we changed the sign convention for $\rho_{i}$ from the one used in Part III.
} 
all potential differences along any cycle must vanish. ${ }^{6}$

Clearly, any flow constructed like equation (D.11) automatically satisfies the mesh rule because of (D.8). In order to satisfy the junction rule, we note that (D.11) can be plugged into (D.12) to obtain the potential vector and from that the flow vector,

$$
\begin{aligned}
\phi & =\left(E K E^{T}\right)^{\dagger} \rho \\
\Rightarrow \mathbf{F} & =K E^{T}\left(E K E^{T}\right)^{\dagger} \rho,
\end{aligned}
$$

where $A^{\dagger}$ is the Moore-Penrose pseudo-inverse of a matrix $A$. It is well known that the nullspace of the weighted Laplacian $L=E K E^{T}$ is spanned by the all-ones vector $\mathbf{1}=(1, \cdots, 1)^{T}$. Therefore we can w.l.o.g. assume $\rho \in \mathcal{V}^{\perp}$ and there exists a (non-unique) vector $\mathbf{u} \in \mathcal{E}$ such that $E \mathbf{u}=\rho$ (because $E$ is an epimorphism). The vector $\mathbf{u}$ is some particular solution to the junction rule (D.12) that does not necessarily satisfy the mesh rule (D.13) as well. Such solutions $\mathbf{u}$ are easy to construct by first writing $\rho$ in the basis given by (D.7) and noting that each basis vector has an expression $\mathbf{b}_{i}=E \mathbf{p}_{1 i}$, where $\mathbf{p}_{1 i} \in \mathcal{E}$ is an oriented path connecting nodes 1 and $i$. See Figure D.1 (c) for an illustration.

This allows us to write the flow projector equation

$$
\begin{aligned}
\mathbf{F} & =K E^{T}\left(E K E^{T}\right)^{\dagger} E \mathbf{u} \\
& =S \mathbf{u} .
\end{aligned}
$$

The matrix $S$ has very interesting properties that can be expressed as a duality between potentials and cycle flows on the network.

Proposition 1. The matrix $S=K E^{T}\left(E K E^{T}\right)^{\dagger} E$ is an oblique (orthogonal if $K=\mathbb{1}$ ) projection on $\mathcal{E}$. Its complementary projection is given by $T=C\left(C^{T} K^{-1} C\right)^{-1} C^{T} K^{-1}$, i.e., we have $S^{2}=S, T^{2}=T, S T=T S=0, S+T=\mathbb{1}$.

Proof. An elementary calculation shows both $S^{2}=S$ and $T^{2}=T$, thus $S$ and $T$ are oblique projectors. If $K=\mathbb{1}, S$ and $T$ are symmetric and therefore orthogonal. We now prove complementarity.

Clearly, $\operatorname{ker} S=\mathcal{C}, \operatorname{im} T \subseteq \mathcal{C}, \operatorname{ker} T=K \mathcal{C}^{\perp}, \operatorname{im} S \subseteq K \mathcal{C}^{\perp}$, where $\mathcal{C}^{\perp}$ is the orthogonal complement of $\mathcal{C}$, the space of cocycles [210]. Because of dimensionality, all the " $\subseteq$ " are actually " $=$ ". Because $E C=0$, we have

$$
S T=0=T S \text {. }
$$

Thus $S$ and $T$ commute and can be diagonalized simultaneously. Because the eigenvalues of projection matrices are either 1 or 0 , and the kernels and images of $S$ and $T$ are exactly complementary, we must have the complementarity relation

$$
S+T=\mathbb{1} \text {. }
$$

\footnotetext{
${ }^{6}$ If $\phi$ represents a set of angles such as in the case of power grids, this rule must be relaxed to allow multiples of $2 \pi$.
} 
We now have two dual methods of calculating the edge flows,

$$
\begin{aligned}
\mathbf{F} & =K E^{T}\left(E K E^{T}\right)^{\dagger} E \mathbf{u} \\
& =\left(\mathbb{1}-C\left(C^{T} K^{-1} C\right)^{-1} C^{T} K^{-1}\right) \mathbf{u} .
\end{aligned}
$$

Note that while the Laplacian $L=E K E^{T}$ is singular and requires the use of a pseudo-inverse, the algebraic dual Laplacian $L^{*}=C^{T} K^{-1} C$ is invertible. The dual form (D.21) has an interesting interpretation. The flow $\mathbf{F}$ is made up out of some particular solution $\mathbf{u}$ of the junction rule that is corrected by cycle flows to satisfy the mesh rule.

\section{D.3 Edge perturbations in linear flow networks}

Consider a solution $\mathbf{F}$ to Kirchhoff's laws on a network with given weights $K$. How does the flow change if we perturb one of the edge weights $K_{e}$ for some edge $\mathbf{e}$ with $K \mathbf{e}=K_{e} \mathbf{e}$ by some amount $\kappa$ while still satisfying the same boundary conditions? Formally,

$$
\begin{aligned}
K_{e} & \rightarrow K_{e}-\kappa \\
\mathbf{F} & \rightarrow \mathbf{F}^{\prime} .
\end{aligned}
$$

We note that the weight matrix changes as $K \rightarrow K-\kappa \mathbf{e e}^{T}$. This is a rank-1 update to the pseudo-inverse of the Laplacian $L^{\dagger}=\left(E K E^{T}\right)^{\dagger} \rightarrow\left(E K E^{T}-\kappa E \mathbf{e e}^{T} E^{T}\right)^{\dagger}$ and we can calculate (D.11) with the new weights using the Sherman-Morrison formula $[218,219]$. After some algebra we find

$$
\mathbf{F}^{\prime}=\mathbf{F} \underbrace{-F_{e} \frac{\kappa}{K_{e}} \frac{(1-S) \mathbf{e}}{1-\kappa R_{e}}}_{=\Delta \mathbf{F}} .
$$

Here, $F_{e}=\mathbf{e}^{T} \mathbf{F}$ and $K_{e}=\mathbf{e}^{T} K \mathbf{e}$ are the original flow and the original weight at the perturbed edge, and $R_{e}=\mathbf{e}^{T} E^{T}\left(E K E^{T}\right)^{\dagger} E \mathbf{e}$ is the resistance distance between the nodes that are connected by $e$. For two arbitrary nodes $i, j$, the resistance distance is defined as $R_{i j}=L_{i i}^{+}+L_{j j}^{+}-2 L_{i j}^{+}$. It is the potential drop measured when injecting a current of strength 1 at node $i$ and of strength -1 at node $j[220,221]$. It is one of the most important network characteristics (see for instance [222]).

We immediately see that this formula has a dual form (using proposition 1),

$$
\Delta \mathbf{F}=-F_{e} \frac{\kappa}{K_{e}} \frac{T \mathbf{e}}{1-\kappa R_{e}} .
$$


The dual form can be obtained alternatively noting that

$$
K^{-1} \rightarrow K^{-1}+\frac{\kappa}{K_{e}\left(K_{e}-\kappa\right)} \mathbf{e e}^{T}
$$

and using the Sherman-Morrison formula with equation (D.21). This also provides an alternative proof of Proposition 1. It is interesting to note that because $S T=0, \mathbf{F}^{T} \Delta \mathbf{F}=0$.

Thus, all possible flows on a weighted flow network and all flow changes due to edge weight perturbations are encoded in the projection matrix $S$ (or equivalently $T$ ). Because for many networks, the number of cycles $|E|-|V|+1$ is much smaller than $|V|$, a considerable computational advantage can be gained from using the dual description of the perturbations in terms of $T$. This is relevant in par-

(a)
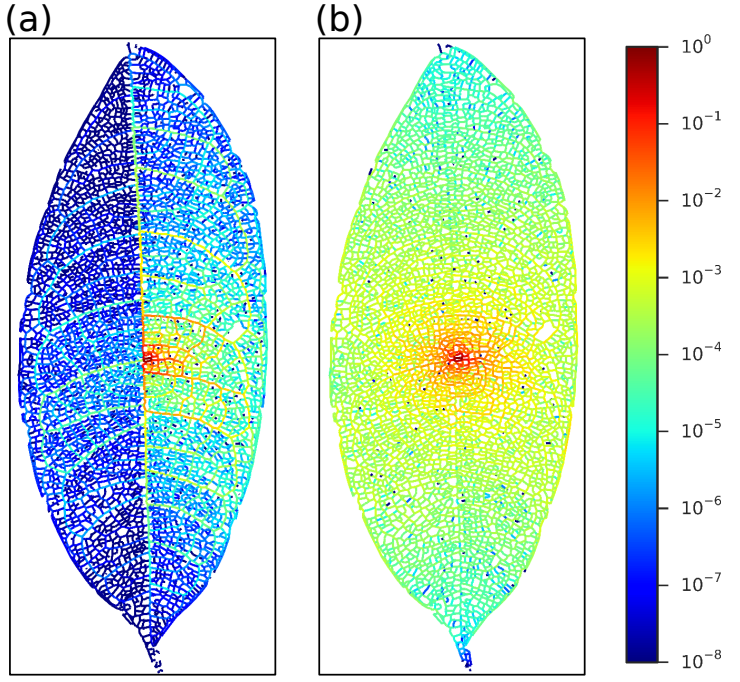

FIGURE D.2: Edge perturbations in a leaf network of Protium grandifolium. Colors correspond to $\left|\Delta \mathbf{F} / \Delta F_{e}\right|$. (a) Realistic leaf network. The hierarchical network localizes the perturbation and reroutes flow through high-conductivity edges. (b) Homogeneous network (all $K_{e}=1$ ). The perturbation appears as a dipole and decays as $r^{-2}$. ticular for power grids [160].

Leaf venation networks are designed in such a way as to efficiently localize perturbations and reroute flow through high-conductivity veins, minimizing the dissipated power due to the flow change (see Figure D.2 (a)).

\section{D.3.1 Power dissipation}

We now show an application of the formalism developed above. In many problems (in particular for fluid flow), one wants to compute the total power dissipation $P$ in the network. It is

$$
P=\mathbf{F}^{T} K^{-1} \mathbf{F} .
$$

In fluid flow networks, this is the power dissipated due to internal viscous friction [223], in electrical resistor networks, it is the thermal dissipation in the resistors. In the perturbed network, we can plug $\mathbf{F}^{\prime}$ and the new $K^{-1}$ into equation (D.27) and obtain

$$
P^{\prime}=P+\underbrace{\frac{\kappa}{K_{e}} \frac{P_{e}}{1-\kappa R_{e}}}_{=\Delta P} .
$$


By expanding $\Delta P$ to order $O(\kappa)$ we also obtain a formula for the partial derivatives

$$
\frac{\partial P}{\partial K_{e}}=-\frac{F_{e}^{2}}{K_{e}^{2}} .
$$

This result is intimately related to Cohn's theorem, but is usually proved in a different way [224].

\section{D.3.2 Cycle flows and decay of perturbations in 2D}

The perturbation flows $\Delta \mathbf{F}$ have two dual descriptions in terms of perturbation potentials $\Delta \phi \in \mathcal{V}$ and cycle flows $\mathbf{f} \in \mathcal{C}$. The expressions are

$$
\begin{aligned}
\mathbf{F}^{\prime} & =\left(K-\kappa \mathbf{e e}^{T}\right) E^{T}(\phi+\Delta \phi) \\
& =\mathbf{F}+\kappa C \mathbf{f},
\end{aligned}
$$

where $\Delta \phi$ and $\mathbf{f}$ satisfy

$$
\begin{aligned}
\left(E K E^{T}\right) \Delta \phi & =\frac{F_{e}}{K_{e}} \frac{E \mathbf{e}}{1-\kappa R_{e}} \\
\left(C^{T} K^{-1} C\right) \mathbf{f} & =-\frac{F_{e}}{K_{e}} \frac{C^{T} \mathbf{e}}{1-\kappa R_{e}} .
\end{aligned}
$$

These expressions can be checked using equations (D.24) and (D.25). We note that the matrices on the left hand side are the Laplacian $L=E K E^{T}$ and the dual Laplacian $L^{*}=C^{T} K^{-1} C$. We now specialize to the case where the network is planar and the choice of basis cycles coincides with the facets of the graph as a planar polygon. Then the right hand sides are dipole source terms.

\section{D.3.3 Decay of perturbations in the continuum approximation}

It is now instructive to construct a continuum approximation for very large networks. The differential equations can be found for a square lattice with lattice spacing $h$ as $h \rightarrow 0$. Because the details are uninteresting, we omit them and directly state the continuum theory. The continuum corresponds to something like a "network density". We write the original flow as a gradient vector field

$$
\mathbf{v}(\mathbf{x})=K(\mathbf{x}) \nabla \varphi(\mathbf{x}),
$$

where $\mathbf{v}(\mathbf{x})$ is the flow, $K(\mathbf{x})$ is a "weight density" and $\varphi(\mathbf{x})$ is the potential. We consider a perturbation of $K(\mathbf{x})$ at position $\mathbf{a}$, changing $\mathbf{v}(\mathbf{x}) \rightarrow \mathbf{v}(\mathbf{x})+\Delta \mathbf{v}(\mathbf{x})$. Assuming constant weights $K(\mathbf{x}) \equiv K$, the continuum version of equation (D.33) 

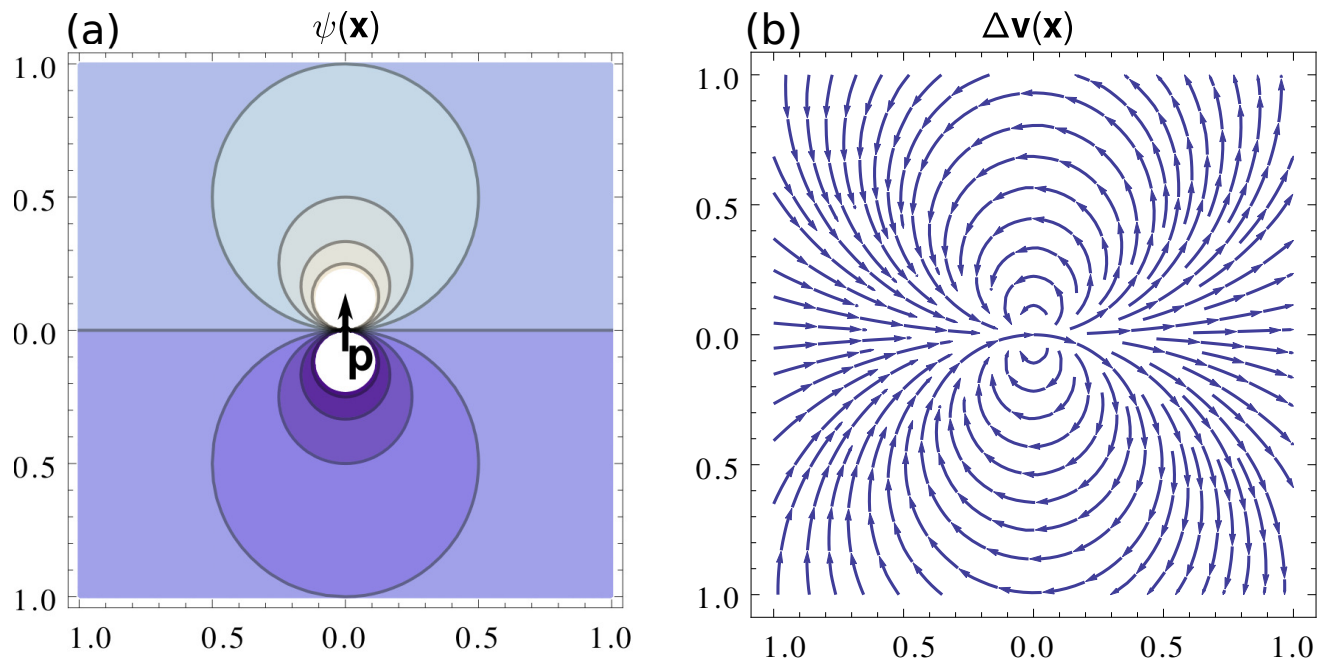

FIGURE D.3: Dipole perturbation in the continuum model on the infinite plane $\mathbb{R}^{2}$. (a) Contour plot of the cycle flow density $\psi(\mathbf{x})$. The vector $\mathbf{p}$ is the cycle dipole moment, which is perpendicular to the perturbed edge and thus to the perturbation flow. (b) Stream plot of the perturbation flow density $\mathbf{v}(\mathbf{x})$.

becomes

$$
\frac{1}{K} \triangle \psi(\mathbf{x})=-\mathbf{p}^{T} \nabla \delta^{(2)}(\mathbf{x}-\mathbf{a}),
$$

where $\psi(\mathbf{x})$ is the "cycle flow density", $K$ is the constant weight density and the right hand side is indeed a dipole source at a with dipole moment $\mathbf{p} \perp \mathbf{v}(\mathbf{a})$. The perturbation flow is

$$
\Delta \mathbf{v}(\mathbf{x})=\left(\begin{array}{cc}
0 & 1 \\
-1 & 0
\end{array}\right) \nabla \psi(\mathbf{x})
$$

and the boundary condition on the cycle potential is such that $\left.\psi\right|_{\partial A}=0$, where $A \subset \mathbb{R}^{2}$. This leads to $\Delta \mathbf{v}$ being parallel to the boundary. These laws are very similar to those of electrostatics (because of the appearance of a $90^{\circ}$ rotation we call them "electrostatics with a twist") and indeed, the solutions are very similar as well. On the infinite plane $\mathbb{R}^{2}$ we find

$$
\begin{aligned}
\psi(\mathbf{x}) & =\frac{\mathbf{p}^{T} \mathbf{x}}{\|x\|^{2}} \sim \frac{1}{r} \\
\|\Delta \mathbf{v}(\mathbf{x})\| & \sim \frac{1}{r^{2}}
\end{aligned}
$$

where $r=\|\mathbf{x}\|$. A plot of both is shown in Figure D.3. Thus, in planar networks that are sufficiently regular and have weights sufficiently close to constant, we expect algebraic decay of the perturbation currents with exponent -2 . This is 
seen even in networks with uniform conductivity but the topology of a leaf network (see Figure D.2 (b)).

As in regular electrostatics, there exists a complex-analytic formulation of the continuum theory. The cycle potential becomes a meromorphic function of $z=x+i y \in \mathbb{C}$. The real potential is given by $\psi(\mathbf{x})=\operatorname{Re}(\psi(z))$ and the physical flow vector can be obtained from the complex potential by

$$
\Delta \mathbf{v}(\mathbf{x})=\left(\begin{array}{l}
+\operatorname{Im}\left(\psi^{\prime}(z)\right) \\
-\operatorname{Re}\left(\psi^{\prime}(z)\right)
\end{array}\right)
$$

This amounts to the vector version of $i \psi^{\prime}(z)$. On the infinite plane, the complex cycle potential is

$$
\psi(z)=\frac{p}{z^{\prime}}
$$

where $p$ is the complex version of the dipole moment $\mathbf{p}$.

\section{D.3.3.1 Boundaries and global topology}

Leaf networks are characterized by being bounded and roughly elliptical in shape. Thus, it is important to study the effects of boundaries and geometry on the decay behavior of perturbations. Similarly, the global topology has an interesting influence on perturbations. Zeros of the perturbation flow appear if the network can be embedded on a sphere or a topological torus. We consider four test cases.

Infinite strip. We consider a network that is an infinite strip in $y$ direction and bounded between $-L / 2$ and $L / 2$ in $x$ direction. Thus, there are two infinite boundaries at $x= \pm L / 2$. Let the perturbation be located at $-L / 2<a<$ $L / 2$, then the cycle potential can be obtained by the method of mirror charges, superposing an infinite number of periodic perturbations. The final result is

$$
\psi(z)=-\frac{\pi}{2 L}\left(p \tan \left(\frac{\pi}{2 L}(z+a)\right)-\bar{p} \cot \left(\frac{\pi}{2 L}(z-a)\right)\right) .
$$

The complex cycle potential has a simple pole at $z=a$ with residue $p$ and its real part vanishes at $z= \pm L / 2+i y$.

Close to the perturbation, $\psi(r) \sim r^{-1}$, where $r$ is the distance to the perturbation. Close to the boundary, $\psi(r) \sim r$, where $r$ is the distance to the boundary. Thus, close to the boundary, the component of $\Delta \mathbf{v}$ parallel to the boundary must tend to a constant. The normal component vanishes because of conservation of total flow. This does not necessarily happen when the flow is not a perturbation because there may be other sources and sinks. 
(a)

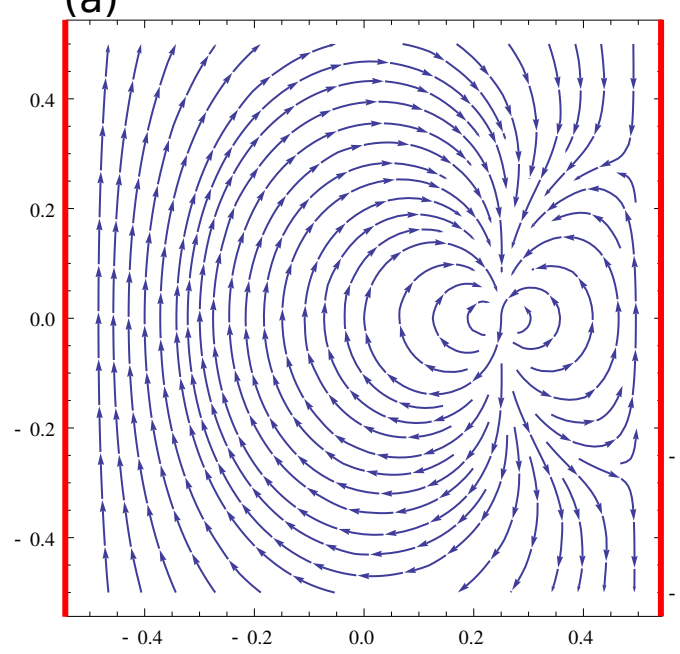

(b)

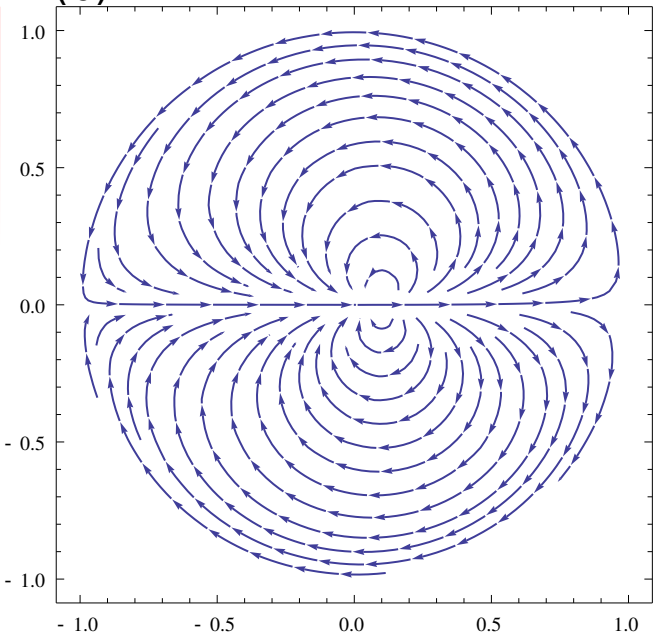

FIGURE D.4: Continuum perturbation flow $\Delta \mathbf{v}(\mathbf{x}) /\|\Delta \mathbf{v}(\mathbf{x})\|$ in two bounded geometries. (a) Infinite strip. Boundaries marked in red. (b) Circular disk. In both cases, it can be seen that the flow is parallel to the boundary.

For a perturbation located at $z=0$ we find

$$
\psi(0, y)=\pi p_{2} \operatorname{csch}(\pi y) .
$$

Thus, in the direction of the infinite strip the perturbation decays exponentially instead of algebraically as on the infinite plane. The appearance of two boundaries has localized the perturbation. A single boundary does not lead to this localization behavior.

Circular disk. The solution for a network with circular boundary is easy to construct from the mirror principle again. The (real) solution for a perturbation located at $a \mathbf{e}_{1}$ and a circle of radius $R$ centered at $(0,0)$ is

$$
\psi(\mathbf{x})=\frac{\mathbf{p}^{T}\left(\mathbf{x}-a \mathbf{e}_{1}\right)}{\left\|\mathbf{x}-a \mathbf{e}_{1}\right\|^{2}}+R^{2} \frac{\mathbf{p}^{T} \mathbf{x}+p_{1} a\|x\|^{2}}{\left\|a \mathbf{x}-R^{2} \mathbf{e}_{1}\right\|^{2}} .
$$

Again, $\psi(r) \sim r^{-1}$ near the perturbation and $\psi(r) \sim r$ near the boundary.

Sphere. The sphere can be stereographically projected onto the complex plane. This is a conformal map, therefore the solution to the Poisson equation on the plane can be simply mapped back to the sphere, resulting in the solution of the corresponding Poisson equation on the sphere. The result in spherical 
(a)

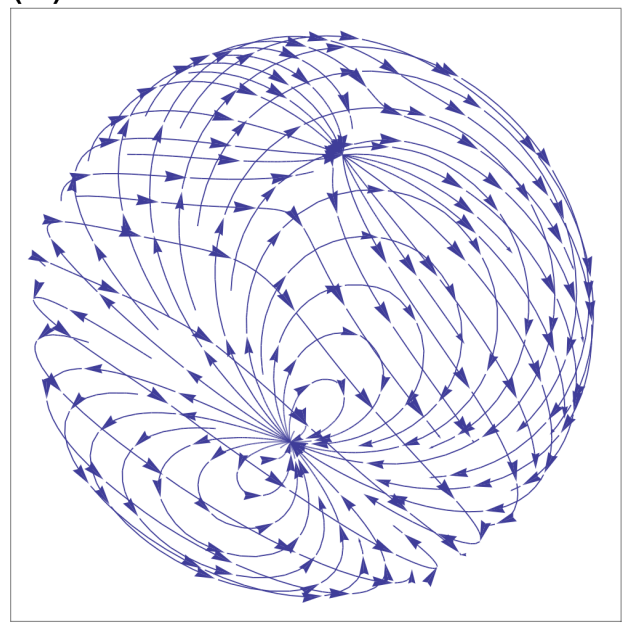

(b)

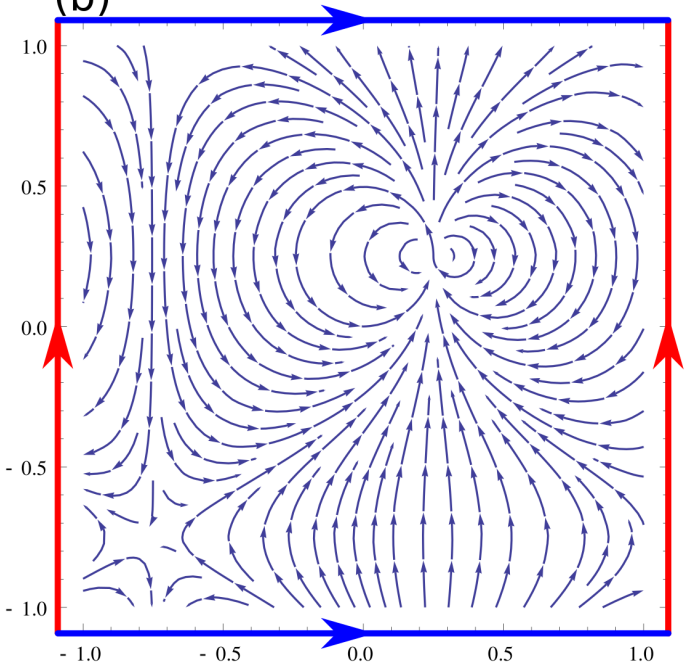

FIGURE D.5: Continuum perturbation flow $\Delta \mathbf{v}(\mathbf{x}) /\|\Delta \mathbf{v}(\mathbf{x})\|$ in two different global topologies. (a) Sphere (genus $g=0$ ). The flow field strength becomes constant at the north pole, opposite of the perturbation site (south pole). (b) Flat torus $(g=1)$ with periods $\omega_{1}=1, \omega_{2}=i$. There is a zero of the flow field in the bottom left. The identified sides of the torus are marked in blue and red.

coordinates for a real dipole moment is

$$
\psi(\theta, \varphi)=4 p \frac{\cos \varphi}{\sin \theta}(1-\cos \theta) .
$$

The perturbation is located at the south pole $(\theta=\pi)$. The factor of 4 comes from the distortion of the area element due to the stereographic projection. The flow field is given by

$$
\Delta \mathbf{v}(\theta, \varphi)=-4 \frac{\sin \varphi}{\sin \theta} \tan \frac{\theta}{2} \mathbf{e}_{\varphi}+2\left(1+\tan ^{2} \frac{\theta}{2}\right) \cos \varphi \mathbf{e}_{\theta} .
$$

It is interesting to note that close to the perturbation, $\psi(\theta, \varphi) \sim \theta^{-1}$ but there is also a zero, $\psi(0, \varphi)=0$. The flow field becomes constant at the north pole, $\|\Delta \mathbf{v}(0, \varphi)\|=p$.

This behavior can be seen on the complex plane, too. Close to the perturbation (corresponding to $\theta=\pi$ ), the complex potential is simply $\psi(z)=p / z$. The neighborhood of infinity $(\theta=0)$ can be reached by the transformation $z \mapsto 1 / z$, resulting in $\psi(z)=p z$.

Torus. Networks that can be embedded on surfaces with nonzero genus (tori with several handles) have been investigated in terms of their hierarchical topology [156], similarly to our work in Part II. The idea is that general networks that do not possess an a priori geometrical embedding can be embedded onto some 
surface of possibly nontrivial genus. The simplest nontrivial such surface is a torus with one hole (A genus $g=1$ surface).

A complex (flat) torus is $\mathbb{C} / \Lambda$, where $\Lambda$ is a lattice spanned by periods $\omega_{1}, \omega_{2} \in$ $\mathbb{C}$ with $\omega_{1} / \omega_{2} \notin \mathbb{R}$. Two points in the complex plane are identified if they differ by a lattice vector.

The naive ansatz for finding the cycle potential would be to try the superposition

$$
\psi(z)=p \sum_{\omega \in \Lambda} \frac{1}{z-\omega}
$$

However, this sum does not converge and is not periodic as required. This can be easily seen by integrating

$$
\oint \psi(z) d z
$$

along the fundamental cell of the torus. Clearly, due to periodicity, the integral must vanish. By the residue theorem it has value $p$ because of the simple pole in the fundamental cell. There is a standard construction to force convergence [225]. One defines

$$
\zeta(z)=\frac{1}{z}+\sum_{\omega \in \Lambda, \omega \neq 0}\left(\frac{1}{z-\omega}+\frac{1}{\omega}+\frac{z}{\omega^{2}}\right) .
$$

This is the Weierstraß $\zeta$-function. Its series representation converges, and it is quasi-periodic. It also possesses the correct poles, and the additional terms vanish under application of the Laplacian. This is the best we can do because in a discrete network with toroidal symmetry, there appears another cycle, in addition to the cycles given by the facet basis, due to the nontrivial topology of the torus (the additional cycle is one of the generators of the torus' fundamental group). This means that the cycle basis of facets on a discrete network with toroidal symmetry is incomplete. This incompleteness is reflected in the fact that no continuous cycle potential exists that satisfies periodicity. The Weierstraß $\zeta$-function is only quasi-periodic $\left(\zeta\left(z+\omega_{i}\right)=\zeta(z)+\eta_{i}\right.$ for some $\eta_{i}$ that depends on the torus periods), similar to the behavior of the complex logarithm.

Note that the physical currents are related to

$$
\zeta^{\prime}(z)=-\wp(z)=-\frac{1}{z^{2}}-\sum_{\omega \in \Lambda, \omega \neq 0}\left(\frac{1}{(z-\omega)^{2}}-\frac{1}{\omega^{2}}\right)
$$

where $\wp(z)$ is the Weierstraß $\wp$-function. This function is periodic and describes the correct physics. Thus, the continuum approximation can still be used to derive interesting facts about the physical flows if one accepts that the continuous cycle potential is unphysical.

One interesting fact is that $\wp(z)$ has zeros. Thus, on a torus, there exist points 
where the flow field vanishes. There is a formula for the location of the zeros [226]. It states that they are located at $\pm z_{0}$, where $z_{0}$ is a generally complicated expression. For $\tau=i$ (i.e., a square lattice torus), the expression is easy to evaluate, yielding $z_{0}=(1+i) / 2$. Because $z_{0} \sim-z_{0}$, this is a double zero.

\section{D.4 Summary}

In this section, we presented a short review of graph theory followed by an account of the theory of linear flow networks. They are used in many applications such as for modeling vascular flow in plants or animals, microfluidics, or power grids. Our variant of the theory features the introduction of a duality between the potential flows (defined at nodes) and the cycle flows (defined on algebraic cycles) making up physical flows (defined on edges). This duality allowed us to derive very compact formulae for the change in flow after perturbing a single edge, the corresponding change in dissipated viscous power as well as its partial derivatives in a particularly easy way. We showed that real leaf networks appear to localize such perturbations by rerouting flow through high-conductivity edges, minimizing dissipated power due to the perturbation. Finally, we sketched the construction of a two-dimensional continuum theory of perturbations (where the continuum represents something like a "network density"), allowing us to derive decay laws for the perturbation strength. The decay laws were then analyzed in two geometrical settings (relevant for leaf venation and similar networks) and two topological settings (relevant for more general networks). 


\section{List of Figures}

2.1 Leaf blade of a dicot . . . . . . . . . . . . . . . . . . . 15

2.2 Leaf interactions with the environment . . . . . . . . . . . . . 16

2.3 A single stoma on a tomato leaf . . . . . . . . . . . . . . . . . . 18

Source: https://commons.wikimedia.org/wiki/File:Tomato_leaf_

stomate_1.jpg

2.4 Gas exchange through a stoma . . . . . . . . . . . . . . . . 19

Source: https://commons.wikimedia.org/wiki/File:HPIM0188-ligusterblad. jpg

2.5 Anatomy of the xylem . . . . . . . . . . . . . . . . . . . 20

Source: https://commons.wikimedia.org/wiki/File:Xylem_cells. svg

2.6 Hydraulic resistor model for a tree . . . . . . . . . . . . . . . . . . . 21

2.7 Phloem anatomy . . . . . . . . . . . . . . . . 23

Source: https://commons.wikimedia.org/wiki/File:Phloem_cells. svg

2.8 Cross section of a dicot leaf . . . . . . . . . . . . . . . 26

Source: https://commons.wikimedia.org/wiki/File:Dicot_leaf_L.

jpg

2.9 Cross section of the vascular bundle . . . . . . . . . . . . 26

Source: https://commons.wikimedia.org/wiki/File:Taraxacum_officinale\% 2C_central_leaf_vein2C_Etzold_green_2.JPG

2.10 Examples of leaf shapes . . . . . . . . . . . . . . . 27

Source: https://commons.wikimedia.org/wiki/File:Leaf_morphology .

svg

2.11 The basic shape of a leaf . . . . . . . . . . . . . . . . 28

2.12 Hierarchical organization of the leaf venation network . . . . . . . 29

2.13 Highest order venation network . . . . . . . . . . . . . . . . . 29

2.14 Simulated damage resistant leaf network . . . . . . . . . . . . 31

2.15 Shoot apical meristem of Coleus sp. . . . . . . . . . . . . . . . 33

Source: https://commons.wikimedia.org/wiki/File:Coleus_stemtip. jpg

2.16 Polar accumulation of PIN in a leaf primordium . . . . . . . . . . . 33

2.17 Development of the vascular pattern in Arabidopsis thaliana . . . . . 34

2.18 Venation types and folded growth . . . . . . . . . . . . . 36

2.19 The angiosperm radiation . . . . . . . . . . . . 38

3.1 Examples of reticulate topology in natural and man-made networks 43 Source: https://commons.wikimedia.org/wiki/File:Physarum_psittacinum. 
3.2 Examples of the diversity of leaf network morphology . . . . . . . 44

3.3 Leaf venation morphogenesis in Arabidopsis thaliana . . . . . . . . . 44

4.1 Partial representation of leaf venation as a weighted planar graph . 48

4.2 Geometric leaf traits . . . . . . . . . . . . . . . . . . . . . . . . 49

4.3 Topological leaf traits . . . . . . . . . . . . . . . . . 50

4.4 Illustration of the pre-processing steps . . . . . . . . . . . . . 51

4.5 Dual graphs for hierarchical decomposition . . . . . . . . . . . . . . 51

4.6 Example hierarchical decomposition . . . . . . . . . . . . . . 52

4.7 Properties of the binary nesting tree . . . . . . . . . . . . . 53

4.8 Topological tapering length and nesting number . . . . . . . . . 55

5.1 Topological degrees of freedom and projection on the first two principal components . . . . . . . . . . . . . . 58

5.2 The first two principal component weights and the first two latent factors loadings for the eight topological and geometrical leaf features 59

5.3 Topological similarity as measured by the KS distance between nesting ratio distributions . . . . . . . . . . . . . . . . 60 60

5.4 Two test leaves together with their two nearest neighbors according to $D_{\mathrm{KS}}$ applied to the nesting ratio statistics . . . . . . . . . . . . 61

5.5 Hierarchical clustering of reduced full leaf data set . . . . . . . . . . 62

5.6 Accuracy estimation of Linear Discriminant Analysis for leaf specimen fragments . . . . . . . . . . . . . . . 63

5.7 Accuracy estimation of Linear Discriminant Analysis for leaf species fragments ......................... 63

5.8 Micro-averaged Receiver Operating Characteristic . . . . . . . . . . 64

6.1 Sketch of the empirical growth model dynamics . . . . . . . . . . 68

6.2 Hierarchical nesting of typical final networks at $N=64 \ldots$. . . . 71

6.3 Phase diagrams of the model without noise . . . . . . . . . . . . . 71

6.4 Phase diagrams of the model with noise . . . . . . . . . . . . . 72

6.5 Comparison of observables in model and data set . . . . . . . . . . 72

6.6 Comparison of statistics between model and a real leaf with high nesting number . . . . . . . . . . . . . . . 73

6.7 Comparison of statistics between model and a real leaf with low nesting number . . . . . . . . . . . . . . . 74

8.1 The levels of coarse-graining appropriate for the analysis of various transportation systems ............... 83

9.1 Phloem geometry in a pine needle . . . . . . . . . . . . . . 88

9.2 Numerical solution of the transport equations . . . . . . . . . . . 90

9.3 Conifer needle characteristics . . . . . . . . . . . . . . . . 92

9.4 Comparison of experimental sieve element area data with prediction from optimization . . . . . . . . . . . . . . 93 
10.1 Simplifying leaf xylem networks . . . . . . . . . . . . . . . . . . 98

10.2 Formation of first loops in damage models . . . . . . . . . . . . 103

10.3 Density of loops as a function of damage . . . . . . . . . . . . . . 104

10.4 Higher order structures near the main vein . . . . . . . . . . . 106

10.5 Higher order structures between two veins . . . . . . . . . . . . 107

10.6 The craspedodromous venation pattern in Carpinus betulus . . . . 108

Source: https://commons.wikimedia.org/wiki/File:Carpinus_betulus2_ ies.jpg

10.7 Typical results from auxin canalization models on a triangular grid 110

10.8 Continuous transition in the loop density . . . . . . . . . . . 112

A.1 Receiver Operating Characteristic curve . . . . . . . . . . . . . . 125

A.2 Principal Component Analysis . . . . . . . . . . . . . . . 130

A.3 The $k$-means algorithm . . . . . . . . . . . . . . . . . . 133

A.4 Hierarchical clustering . . . . . . . . . . . . . . . . . . 134

A.5 Linear Discriminant Analysis . . . . . . . . . . . . . . 136

B.1 The vectorization process . . . . . . . . . . . . . . . 139

B.2 Detecting fundamental cycles . . . . . . . . . . . . . . . . . . 140

B.3 Traversing in the left-most direction . . . . . . . . . . . . 141

C.1 Nesting tree model topologies . . . . . . . . . . . . . . . . 143

D.1 Oriented graphs and flows . . . . . . . . . . . . . . . . . . 148

D.2 Edge perturbations in a real leaf network . . . . . . . . . . . . . 154

D.3 Dipole perturbation in the continuum model . . . . . . . . . . . . 156

D.4 Continuum perturbation flow on an infinite strip and on a circle . . 158

D.5 Continuum perturbation flow on a sphere and on a torus . . . . . . 159 


\section{Bibliography}

[1] F. Hallé. In Praise of Plants. Portland, Oregon: Timber Press, Inc., 2002. ISBN: 978-1-60469262-4.

[2] P. H. Raven, R. F. Evert, and S. E. Eichhorn. Biology of Plants. Eighth. New York, NY: W. H. Freeman, 2013. ISBN: 9780716710073.

[3] S. Vogel. The Life of a Leaf. Chicago, London: University of Chicago Press, 2012. IsBN: 9780226104775. DOI: 10.7208/chicago/9780226859422.001.0001.

[4] A. J. McElrone, B. Choat, G. A. Gambetta, and C. R. Brodersen. "Water Uptake and Transport in Vascular Plants". Nature Education Knowledge 4.5 (2013).

[5] L. Sack and N. M. Holbrook. "Leaf Hydraulics". Annual Review of Plant Biology 57.1 (2006), pp. 361-381. ISSN: 1543-5008. DOI: 10.1146/annurev . arplant . 56.032604. 144141.

[6] I. N. Forseth. "The Ecology of Photosynthetic Pathways". Nature Education Knowledge 3.10 (2010).

[7] G. S. Singhal. Concepts in Photobiology: Photosynthesis and Photomorphogenesis. Kluwer Academic Publishers, 1999. ISBN: 9780792355199.

[8] A. Laisk and V. Oja. Dynamics of Leaf Photosynthesis: Rapid-response Measurements and Their Interpretations. Techniques in Plant Sciences. CSIRO Publishing, 1998. ISBN: 9780643063785.

[9] E. G. Nisbet and R. E. R. Nisbet. "Methane, oxygen, photosynthesis, rubisco and the regulation of the air through time". Philosophical Transactions of the Royal Society B: Biological Sciences 363.1504 (2008), pp. 2745-2754. ISSN: 0962-8436. DOI: 10 . 1098 / rstb. 2008 . 0057.

[10] R. Buick. "When did oxygenic photosynthesis evolve?" Philosophical Transactions of the Royal Society B: Biological Sciences 363.1504 (2008), pp. 2731-2743. ISSN: 0962-8436. DOI: $10.1098 /$ rstb.2008.0041.

[11] D. S. Bendall, C. J. Howe, E. G. Nisbet, and R. E. R. Nisbet. "Photosynthetic and atmospheric evolution. Introduction." Philosophical transactions of the Royal Society of London. Series B, Biological sciences 363.1504 (2008), pp. 2625-2628. ISSN: 0962-8436. DOI: $10.1098 /$ rstb. 2008.0058 .

[12] A. Dhingra, A. R. Portis, and H. Daniell. "Enhanced translation of a chloroplast-expressed $\mathrm{RbcS}$ gene restores small subunit levels and photosynthesis in nuclear RbcS antisense plants". Proceedings of the National Academy of Sciences 101.16 (2004), pp. 6315-6320. ISSN: 0027-8424. DOI: 10.1073 /pnas . 0400981101.

[13] E. Steudle. "The Cohesion-Tension Mechanism and Acquisition of Water by Plant Roots". Annual Review of Plant Physiology and Plant Molecular Biology 52.1 (2001), pp. 847-875. ISSN: 1040-2519. DOI: 10.1146/annurev . arplant.52.1.847.

[14] M. T. Tyree and M. H. Zimmermann. Xylem Structure and the Ascent of Sap. Springer Series in Wood Science. Berlin, Heidelberg: Springer Berlin Heidelberg, 2002. ISBN: 978-3-64207768-5. DOI: $10.1007 / 978-3-662-04931-0$. 
Bibliography

[15] J. S. Sperry, N. M. Holbrook, M. H. Zimmermann, and M. T. Tyree. "Spring Filling of Xylem Vessels in Wild Grapevine". Plant Physiology 83.2 (1987), pp. 414-417. ISSN: 0032-0889. DOI: 10.1104 /pp.83.2.414.

[16] J. Böhm. "Capillarität und Saftsteigen”. Berichte der Deutschen Botanischen Gesellschaft 11 (1893), pp. 203-212.

[17] P. J. Melcher, F. C. Meinzer, D. E. Yount, G. Goldstein, and U. Zimmermann. “Comparative measurements of xylem pressure in transpiring and non-transpiring leaves by means of the pressure chamber and the xylem pressure probe". Journal of Experimental Botany 49.327 (1998), pp. 1757-1760. ISSN: 0022-0957. DOI: 10.1093 / jxb / 49.327 .1757$.

[18] J. S. Sperry and M. T. Tyree. "Mechanism of Water Stress-Induced Xylem Embolism". Plant Physiology 88.3 (1988), pp. 581-587. ISSN: 0032-0889. DOI: 10.1104 /pp. 88.3 .581$.

[19] F. C. Meinzer and K. A. McCulloh. "Xylem recovery from drought-induced embolism: where is the hydraulic point of no return?" Tree Physiology 33.4 (2013), pp. 331-334. ISSN: 0829-318X. DOI: $10.1093 /$ treephys/tpt 022.

[20] T. D. Wheeler and A. D. Stroock. "The transpiration of water at negative pressures in a synthetic tree". Nature 455.7210 (2008), pp. 208-212. ISSN: 0028-0836. DOI: 10.1038 / nature07226.

[21] H. Darcy. Les Fontaines Publiques de la Ville de Dijon. Paris: Dalmont, 1856.

[22] B. J. Kirby. Micro- and Nanoscale Fluid Mechanics: Transport in Microfluidic Devices. Cambridge University Press, 2010. ISBN: 978-0-521-11903-0.

[23] D. J. Ellerby and A. R. Ennos. "Resistances to fluid flow of model xylem vessels with simple and scalariform perforation plates". Journal of Experimental Botany 49.323 (1998), pp. 979-985. ISSN: 0022-0957. DOI: $10.1093 / \mathrm{jxb} / 49.323 .979$.

[24] H. R. Brown. "The Theory of the Rise of Sap in Trees: Some Historical and Conceptual Remarks". Physics in Perspective 15.3 (2013), pp. 320-358. ISSN: 1422-6944. DOI: $10.1007 /$ s00016-013-0117-1.

[25] U. G. Hacke, J. S. Sperry, J. K. Wheeler, and L. Castro. "Scaling of angiosperm xylem structure with safety and efficiency". Tree Physiology 26.6 (2006), pp. 689-701. ISSN: 0829318X. DOI: $10.1093 /$ treephys/26.6.689.

[26] B. Choat, A. R. Cobb, and S. Jansen. "Structure and function of bordered pits: new discoveries and impacts on whole-plant hydraulic function". New Phytologist 177.3 (2008), pp. 608-626. ISSN: 0028646X. DOI: 10.1111/j.1469-8137.2007.02317.x.

[27] V. De Schepper, T. De Swaef, I. Bauweraerts, and K. Steppe. "Phloem transport: a review of mechanisms and controls". Journal of Experimental Botany 64.16 (2013), pp. 4839-4850. ISSN: 0022-0957. DOI: $10.1093 / \mathrm{jxb} / \mathrm{ert} 302$.

[28] A. Schulz and G. A. Thompson. "Phloem Structure and Function". In: Encyclopedia of Life Sciences. Vol. September. Chichester, UK: John Wiley \& Sons, Ltd, 2009. DOI: $10.1002 /$ 9780470015902.a0001290.pub2.

[29] M. Knoblauch and K. Oparka. "The structure of the phloem - still more questions than answers". The Plant Journal 70.1 (2012), pp. 147-156. ISSN: 09607412. DOI: 10.1111 / j . 1365-313x.2012.04931.x.

[30] K. H. Jensen, J. Liesche, T. Bohr, and A. Schulz. "Universality of phloem transport in seed plants". Plant, Cell \& Environment 35.6 (2012), pp. 1065-1076. ISSN: 01407791. DOI: $10.1111 / j .1365-3040.2011 .02472$.x. 
[31] D. L. Mullendore, C. W. Windt, H. Van As, and M. Knoblauch. "Sieve Tube Geometry in Relation to Phloem Flow". The Plant Cell 22.3 (2010), pp. 579-593. ISSN: 1040-4651. DOI: $10.1105 /$ tpc.109.070094.

[32] K. H. Jensen et al. "Modeling the Hydrodynamics of Phloem Sieve Plates". Frontiers in Plant Science 3.July (2012), p. 151. ISSN: 1664-462X. DOI: 10.3389/fpls. 2012.00151.

[33] D. R. Froelich et al. "Phloem Ultrastructure and Pressure Flow: Sieve-Element-OcclusionRelated Agglomerations Do Not Affect Translocation". The Plant Cell 23.12 (2011), pp. 44284445. ISSN: 1040-4651. DOI: $10.1105 /$ tpc.111.093179.

[34] M. Knoblauch, D. R. Froelich, W. F. Pickard, and W. S. Peters. "SEORious business: structural proteins in sieve tubes and their involvement in sieve element occlusion". Journal of Experimental Botany 65.7 (2014), pp. 1879-1893. ISSN: 0022-0957. DOI: 10.1093 / jxb/eruo71.

[35] J. S. Kennedy and T. E. Mittler. "A Method of obtaining Phloem Sap via the Mouth-parts of Aphids". Nature 171.4351 (1953), pp. 528-528. ISSN: 0028-0836. DOI: 10 . 1038/171528a 0.

[36] E. A. Rennie and R. Turgeon. "A comprehensive picture of phloem loading strategies". Proceedings of the National Academy of Sciences 106.33 (2009), pp. 14162-14167. ISSN: 00278424. DOI: $10.1073 /$ pnas. 0902279106.

[37] E. Münch. Die Stoffbewegungen in der Pflanze. Jena: Verlag von Gustav Fischer, 1930.

[38] P. Cabrita, M. Thorpe, and G. Huber. "Hydrodynamics of steady state phloem transport with radial leakage of solute". Frontiers in Plant Science 4.December (2013), p. 531. ISSN: 1664-462X. DOI: $10.3389 / \mathrm{fpls} .2013 .00531$.

[39] K. H. Jensen and M. A. Zwieniecki. "Physical Limits to Leaf Size in Tall Trees". Physical Review Letters 110.1 (2013), p. 018104. ISSN: 0031-9007. DOI: 10.1103 / PhysRevLett. 110.018104.

[40] R. Turgeon. “The Role of Phloem Loading Reconsidered". Plant Physiology 152.4 (2010), pp. 1817-1823. ISSN: 0032-0889. DOI: 10.1104/pp.110.153023.

[41] R. Turgeon. "The Puzzle of Phloem Pressure". Plant Physiology 154.2 (2010), pp. 578-581. ISSN: 0032-0889. DOI: $10.1104 / \mathrm{pp} .110 .161679$.

[42] K. H. Jensen et al. "Optimality of the Munch mechanism for translocation of sugars in plants". Journal of The Royal Society Interface 8.61 (2011), pp. 1155-1165. ISSN: 1742-5689. DOI: $10.1098 /$ rsif.2010.0578.

[43] K. H. Jensen, K. Berg-Sørensen, S. M. Friis, and T. Bohr. "Analytic solutions and universal properties of sugar loading models in Münch phloem flow". Journal of Theoretical Biology 304.0 (2012), pp. 286-296. ISSN: 00225193. DOI: $10.1016 / j \cdot j t b i .2012 .03 .012$.

[44] W. F. Pickard and B. Abraham-Shrauner. "A simplest steady-state Munch-like model of phloem translocation, with source and pathway and sink". Functional Plant Biology 36.7 (2009), p. 629. ISSN: 1445-4408. DOI: 10 .1071/FP 08278.

[45] H. Ronellenfitsch et al. "Scaling of phloem structure and optimality of photoassimilate transport in conifer needles". Proceedings of the Royal Society B: Biological Sciences 282.1801 (2015), p. 20141863. ISSN: 0962-8452. DOI: 10 . 1098 / rspb. 2014.1863. arXiv: 1412 . 1272.

[46] R. Turgeon and R. Medville. "The absence of phloem loading in willow leaves". Proceedings of the National Academy of Sciences 95.20 (1998), pp. 12055-12060. ISSN: 0027-8424. DOI: $10.1073 /$ pnas.95.20.12055. 
Bibliography

[47] J. Liesche, H. J. Martens, and A. Schulz. "Symplasmic transport and phloem loading in gymnosperm leaves". Protoplasma 248.1 (2011), pp. 181-190. ISSN: 0033-183X. DOI: 10.1007/s00709-010-0239-0.

[48] J. H. Jung and C. M. Park. "Vascular development in plants: specification of xylem and phloem tissues". Journal of Plant Biology 50.3 (2007), pp. 301-305. ISSN: 1226-9239. DOI: $10.1007 / \mathrm{BF} 03030658$.

[49] L. Sack and C. Scoffoni. "Leaf venation: structure, function, development, evolution, ecology and applications in the past, present and future". New Phytologist 198.4 (2013), pp. 983-1000. ISSN: 0028646X. DOI: 10.1111/nph.12253.

[50] M. Reusche et al. "Verticillium Infection Triggers VASCULAR-RELATED NAC DOMAIN7Dependent de Novo Xylem Formation and Enhances Drought Tolerance in Arabidopsis". The Plant Cell 24.9 (2012), pp. 3823-3837. ISSN: 1040-4651. DOI: 10 . 1105 / tpc . 112. 103374.

[51] B. Ellis, D. C. Daly, and L. J. Hickey. Manual of Leaf Architecture. Cornell University Press, 2009. ISBN: 978-0801475184.

[52] A. B. Nicotra et al. "The evolution and functional significance of leaf shape in the angiosperms". Functional Plant Biology 38.7 (2011), p. 535. ISSN: 1445-4408. DOI: 10.1071 / FP11057.

[53] G. B. West, J. H. Brown, and B. J. Enquist. "A General Model for the Origin of Allometric Scaling Laws in Biology". Science 276.5309 (1997), pp. 122-126. ISSN: 00368075. DOI: $10.1126 /$ science.276.5309.122.

[54] G. B. West, J. H. Brown, and B. J. Enquist. "A general model for the structure and allometry of plant vascular systems". Nature 400.6745 (1999), pp. 664-667. ISSN: 00280836. DOI: $10.1038 / 23251$.

[55] C. A. Price, B. J. Enquist, and V. M. Savage. "A general model for allometric covariation in botanical form and function". Proceedings of the National Academy of Sciences 104.32 (2007), pp. 13204-13209. ISSN: 0027-8424. DOI: 10.1073 /pnas . 0702242104.

[56] W. A. Green et al. "Reading the Leaves: A Comparison of Leaf Rank and Automated Areole Measurement for Quantifying Aspects of Leaf Venation". Applications in Plant Sciences 2.8 (2014), p. 1400006. ISSN: 2168-0450. DOI: 10.3732/apps . 1400006.

[57] L. J. Hickey. "Classification of the Architecture of Dicotyledonous Leaves". American Journal of Botany 60.1 (1973), p. 17. ISSN: 00029122. DOI: 10.2307/2441319.

[58] E. Katifori and M. O. Magnasco. "Quantifying Loopy Network Architectures". PLoS ONE 7.6 (2012). Ed. by J. Bourdon, e37994. ISSN: 1932-6203. DOI: 10 . 1371 / journal . pone. 0037994 . arXiv: 1110.1412.

[59] Y. Mileyko, H. Edelsbrunner, C. A. Price, and J. S. Weitz. "Hierarchical Ordering of Reticular Networks". PLoS ONE 7.6 (2012). Ed. by J. Bourdon, e36715. ISSN: 1932-6203. DOI: $10.1371 /$ journal.pone.0036715. arXiv: $1110.1413 \mathrm{v} 1$.

[60] I. J. Wright et al. "The worldwide leaf economics spectrum". Nature 428.6985 (2004), pp. 821-827. ISSN: 0028-0836. DOI: 10.1038 / nature 02403.

[61] I. J. Wright et al. "Modulation of leaf economic traits and trait relationships by climate". Global Ecology and Biogeography 14.5 (2005), pp. 411-421. ISSN: 1466822X. DOI: $10.1111 /$ j.1466-822x.2005.00172.x. 
[62] B. Blonder, C. Violle, L. P. Bentley, and B. J. Enquist. "Venation networks and the origin of the leaf economics spectrum". Ecology Letters 14.2 (2011), pp. 91-100. ISSN: 1461023X. DOI: 10.1111/j.1461-0248.2010.01554.x.

[63] L. Sack et al. "Developmentally based scaling of leaf venation architecture explains global ecological patterns". Nature Communications 3.May (2012), p. 837. ISSN: 2041-1723. DOI: $10.1038 /$ ncomms 1835.

[64] A. D. McKown, H. Cochard, and L. Sack. "Decoding Leaf Hydraulics with a Spatially Explicit Model: Principles of Venation Architecture and Implications for Its Evolution". The American Naturalist 175.4 (2010), pp. 447-460. ISSN: 0003-0147. DOI: $10.1086 / 650721$.

[65] C. D. Murray. "The Physiological Principle of Minimum Work: I. The Vascular System and the Cost of Blood Volume". Proceedings of the National Academy of Sciences 12.3 (1926), pp. 207-214. ISSN: 0027-8424. DOI: 10.1073/pnas.12.3.207.

[66] C. D. Murray. “The Physiological Principle of Minimum Work: II. Oxygen Exchange in Capillaries". Proceedings of the National Academy of Sciences 12.5 (1926), pp. 299-304. ISSN: 0027-8424. DOI: $10.1073 /$ pnas.12.5.299.

[67] J. Richter. The notebooks of Leonardo da Vinci. New York: Dover, 1970. ISBN: 978-1453772072.

[68] A. Roth-Nebelsick, D. Uhl, V. Mosbrugger, and H. Kerp. "Evolution and Function of Leaf Venation Architecture: A Review". Annals of Botany 87.5 (2001), pp. 553-566. ISSN: 03057364. DOI: $10.1006 /$ anbo.2001.1391.

[69] K. A. McCulloh, J. S. Sperry, and F. R. Adler. "Water transport in plants obeys Murray's law". Nature 421.6926 (2003), pp. 939-942. ISSN: 0028-0836. DOI: 10.1038 / nature 01444.

[70] V. Mosbrugger and A. Roth. "Biomechanics in fossil plant biology". Review of Palaeobotany and Palynology 90.3-4 (1996), pp. 195-207. ISSN: 00346667. DOI: 10 . $1016 / 0034-$ $6667(95) 00083-6$.

[71] M. A. Zwieniecki, H. A. Stone, A. Leigh, C. K. Boyce, and N. M. Holbrook. "Hydraulic design of pine needles: one-dimensional optimization for single-vein leaves". Plant, Cell and Environment 29.5 (2006), pp. 803-809. ISSN: 0140-7791. DOI: $10.1111 / \mathrm{j} .1365-$ $3040.2005 .01448 . x$.

[72] E. Katifori, G. J. Szöllősi, and M. O. Magnasco. “Damage and Fluctuations Induce Loops in Optimal Transport Networks". Physical Review Letters 104.4 (2010), p. 048704. ISSN: 0031-9007. DOI: 10.1103/PhysRevLett.104.048704. arXiv: 0906.0006.

[73] R. Dawkins. Climbing mount improbable. Penguin Books Limited, 1996, p. 349. ISBN: 0393039307. DOI: $10.5860 /$ CHOICE. 34-4466.

[74] A. Bejan, S. Lorente, and J. Lee. "Unifying constructal theory of tree roots, canopies and forests". Journal of Theoretical Biology 254.3 (2008), pp. 529-540. ISSN: 00225193. DOI: $10.1016 / j . j$ tbi.2008.06.026.

[75] A. Bejan and S. Lorente. "The constructal law of design and evolution in nature." Philosophical transactions of the Royal Society of London. Series B, Biological sciences 365.1545 (2010), pp. 1335-1347. ISSN: 0962-8436. DOI: 10.1098 /rstb. 2009.0302.

[76] A. Bejan. "The constructal law of organization in nature: tree-shaped flows and body size". Journal of Experimental Biology 208.9 (2005), pp. 1677-1686. ISSN: 0022-0949. DOI: $10.1242 /$ jeb.01487.

[77] M. Kleiber. "Body size and metabolism". Hilgardia 6.11 (1932), pp. 315-353. ISSN: 00732230. DOI: $10.3733 / \mathrm{hilg} \cdot \mathrm{v} 06 \mathrm{n} 11 \mathrm{p} 315$. 
Bibliography

[78] J. Dumais and D. Kwiatkowska. "Analysis of surface growth in shoot apices". The Plant Journal 31.2 (2002), pp. 229-241. ISSN: 0960-7412. DOI: $10.1046 /$ j.1365-313X. 2001. $01350 . x$.

[79] S. Stoma, J. Chopard, C. Godin, and J. Traas. "Using mechanics in the modelling of meristem morphogenesis". In: 5th International Workshop on Functional-Structural Plant Models. Napier, New Zealand, 2007, pp. 52-1-4.

[80] J. Dumais and C. R. Steele. "New Evidence for the Role of Mechanical Forces in the Shoot Apical Meristem". Journal of Plant Growth Regulation 19.1 (2000), pp. 7-18. ISSN: 0721-7595. DOI: $10.1007 / \mathrm{s} 003440000003$.

[81] D. Kwiatkowska. "Growth and morphogenesis at the vegetative shoot apex of Anagallis arvensis L." Journal of Experimental Botany 54.387 (2003), pp. 1585-1595. ISSN: 14602431. DOI: $10.1093 / \mathrm{jxb} / \mathrm{erg} 166$.

[82] D. Reinhardt et al. "Regulation of phyllotaxis by polar auxin transport". Nature 426.6964 (2003), pp. 255-260. ISSN: 0028-0836. DOI: 10.1038 / nature02081.

[83] J. A. H. Murray, A. Jones, C. Godin, and J. Traas. "Systems Analysis of Shoot Apical Meristem Growth and Development: Integrating Hormonal and Mechanical Signaling". The Plant Cell 24.10 (2012), pp. 3907-3919. ISSN: 1040-4651. DOI: 10.1105 / tpc . 112. 102194.

[84] T. Bennett, G. Hines, and O. Leyser. "Canalization: what the flux?" Trends in Genetics 30.2 (2014), pp. 41-48. ISSN: 01689525. DOI: 10.1016/j.tig.2013.11.001.

[85] K. van Berkel, R. J. de Boer, B. Scheres, and K. ten Tusscher. "Polar auxin transport: models and mechanisms". Development 140.11 (2013), pp. 2253-2268. ISSN: 0950-1991. DOI: $10.1242 / \mathrm{dev} .079111$.

[86] J. Kang and N. Dengler. "Vein Pattern Development in Adult Leaves of Arabidopsis thaliana". International Journal of Plant Sciences 165.2 (2004), pp. 231-242. ISSN: 1058-5893. DOI: $10.1086 / 382794$.

[87] P. Dimitrov and S. W. Zucker. "A constant production hypothesis guides leaf venation patterning". Proceedings of the National Academy of Sciences 103.24 (2006), pp. 9363-9368. ISSN: 0027-8424. DOI: 10.1073 /pnas . 0603559103.

[88] T. Sachs. "Cell polarity and tissue patterning in plants". Development 113 (1991), pp. 83-93. ISSN: 0950-1991.

[89] E. Scarpella. "Control of leaf vascular patterning by polar auxin transport". Genes $\mathcal{E}$ Development 20.8 (2006), pp. 1015-1027. ISSN: 0890-9369. DOI: $10.1101 / \mathrm{gad} .1402406$.

[90] E. Scarpella, M. Barkoulas, and M. Tsiantis. "Control of Leaf and Vein Development by Auxin". Cold Spring Harbor Perspectives in Biology 2.1 (2010), a001511-a001511. ISSN: 1943-0264. DOI: $10.1101 /$ cshperspect . a001511.

[91] D. Marcos and T. Berleth. "Dynamic auxin transport patterns preceding vein formation revealed by live-imaging of Arabidopsis leaf primordia". Frontiers in Plant Science 5.June (2014), p. 235. ISSN: 1664-462X. DOI: 10.3389 /fpls . 2014.00235.

[92] F. A. Ditengou et al. "The role of auxin flux independent mechanisms in regulation of leaf vein initiation and patterning". In preparation (2015).

[93] M. F. Laguna, S. Bohn, and E. A. Jagla. "The Role of Elastic Stresses on Leaf Venation Morphogenesis". PLoS Computational Biology 4.4 (2008). Ed. by P. E. Bourne, e1000055. ISSN: 1553-7358. DOI: 10.1371 / journal. pcbi. 1000055 . arXiv: 0705.0902. 
[94] S. Bohn, B. Andreotti, S. Douady, J. Munzinger, and Y. Couder. "Constitutive property of the local organization of leaf venation networks". Physical Review E 65.6 (2002), p. 061914. ISSN: 1063-651X. DOI: 10.1103/PhysRevE.65.061914.

[95] Y. Couder, L. Pauchard, C. Allain, M. Adda-Bedia, and S. Douady. "The leaf venation as formed in a tensorial field". The European Physical Journal B 28.2 (2002), pp. 135-138. ISSN: 1434-6028. DOI: $10.1140 /$ epjb/e2002-00211-1.

[96] G. J. Mitchison. "A Model for Vein Formation in Higher Plants". Proceedings of the Royal Society B: Biological Sciences 207.1166 (1980), pp. 79-109. ISSN: 0962-8452. DOI: $10.1098 /$ rspb.1980.0015.

[97] A.-G. Rolland-Lagan and P. Prusinkiewicz. "Reviewing models of auxin canalization in the context of leaf vein pattern formation in Arabidopsis". The Plant Journal 44.5 (2005), pp. 854-865. ISSN: 09607412. DOI: $10.1111 / j .1365-313 x .2005 .02581 . x$.

[98] K. Alim and E. Frey. "Quantitative predictions on auxin-induced polar distribution of PIN proteins during vein formation in leaves". European Physical Journal E 33.2 (2010), pp. 165173. ISSN: 1292-895X. DOI: $10.1140 /$ ep je/i2010-10604-5. arXiv: 1005.3768.

[99] F. G. Feugier and Y. Iwasa. "How canalization can make loops: A new model of reticulated leaf vascular pattern formation". Journal of Theoretical Biology 243.2 (2006), pp. 235-244. ISSN: 00225193. DOI: $10.1016 / j$.jtbi.2006.05.022.

[100] R. M. Merks, Y. Van de Peer, D. Inzé, and G. T. Beemster. "Canalization without flux sensors: a traveling-wave hypothesis". Trends in Plant Science 12.9 (2007), pp. 384-390. ISSN: 13601385. DOI: $10.1016 / \mathrm{j}$.tplants.2007.08.004.

[101] B. F. Wilson. The Growing Tree. University of Massachusetts Press, 1984, p. 138. ISBN: 9780870234248 .

[102] E. Couturier, N. Brunel, S. Douady, and N. Nakayama. "Abaxial growth and steric constraints guide leaf folding and shape in Acer pseudoplatanus". American Journal of Botany 99.8 (2012), pp. 1289-1299. ISSN: 0002-9122. DOI: 10 . 3732 / a jb. 1100325.

[103] E. Couturier, S. Courrech du Pont, and S. Douady. "The filling law: A general framework for leaf folding and its consequences on leaf shape diversity". Journal of Theoretical Biology 289 (2011), pp. 47-64. ISSN: 00225193. DOI: 10.1016/j. jtbi.2011.08.020.

[104] E. Couturier, S. Courrech du Pont, and S. Douady. "A Global Regulation Inducing the Shape of Growing Folded Leaves". PLoS ONE 4.11 (2009). Ed. by S. Humphries, e7968. ISSN: 1932-6203. DOI: 10.1371 / journal.pone.0007968.

[105] N. Dengler and J. Kang. "Vascular patterning and leaf shape". Current Opinion in Plant Biology 4.1 (2001), pp. 50-56. ISSN: 13695266. DOI: 10 .1016/S1369-5266 (00) $00135-$ 7.

[106] H. Fujita and A. Mochizuki. "The origin of the diversity of leaf venation pattern". Developmental Dynamics 235.10 (2006), pp. 2710-2721. ISSN: 10588388. DOI: 10.1002 / dvdy . 20908.

[107] S. B. Schmerler et al. "Evolution of leaf form correlates with tropical-temperate transitions in Viburnum (Adoxaceae)". Proceedings of the Royal Society B: Biological Sciences 279.1744 (2012), pp. 3905-3913. ISSN: 0962-8452. DOI: $10.1098 /$ rspb . 2012.1110.

[108] W. B. Critchfield. "Leaf Dimorphism in Populus Trichocarpa". American Journal of Botany 47.8 (1960), p. 699. ISSN: 00029122. DOI: $10.2307 / 2439521$.

[109] J. S. Sperry. "Evolution of Water Transport and Xylem Structure". International Journal of Plant Sciences 164.S3 (2003), pp. 115-127. DOI: $10.1086 / 368398$. 
Bibliography

[110] M. L. Trivett and K. B. Pigg. "A Survey of Reticulate Venation Among Fossil and Living Land Plants". In: Flowering Plant Origin, Evolution \& Phylogeny. 1970. Boston, MA: Springer US, 1996, pp. 8-31. ISBN: 978-0-585-23095-5. DOI: 10 .1007/978-0-585-23095-5_2.

[111] H. J. de Boer, M. B. Eppinga, M. J. Wassen, and S. C. Dekker. "A critical transition in leaf evolution facilitated the Cretaceous angiosperm revolution". Nature Communications 3.May (2012), p. 1221. ISSN: 2041-1723. DOI: 10.1038 /ncomms 2217.

[112] W. E. Friedman. "The meaning of Darwin's 'abominable mystery'". American Journal of Botany 96.1 (2009), pp. 5-21. ISSN: 0002-9122. DOI: 10.3732 / a jb. 0800150.

[113] J. A. Doyle. "Origin of Angiosperms". Annual Review of Ecology and Systematics 9.1 (1978), pp. 365-392. ISSN: 0066-4162. DOI: 10.1146/annurev.es.09.110178.002053.

[114] F. Corson. "Fluctuations and Redundancy in Optimal Transport Networks". Physical Review Letters 104.4 (2010), p. 048703. ISSN: 0031-9007. DOI: 10 . 1103 / PhysRevLett. 104.048703. arXiv: 0905.4947.

[115] F. Costard, L. Dupeyrat, E. Gautier, and E. Carey-Gailhardis. "Fluvial thermal erosion investigations along a rapidly eroding river bank: application to the Lena River (central Siberia)". Earth Surface Processes and Landforms 28.12 (2003), pp. 1349-1359. ISSN: 0197-9337. DOI: 10.1002 /esp. 592.

[116] D. Bi, J. Zhang, B. Chakraborty, and R. P. Behringer. "Jamming by shear". Nature 480.7377 (2011), pp. 355-358. ISSN: 0028-0836. DOI: 10 .1038/nature10667.

[117] A. J. Zomorodian. Topology for Computing. Cambridge Monographs on Applied and Computational Mathematics. Cambridge: Cambridge University Press, 2005, p. 258. ISBN: 9780511546945. DOI: $10.1017 /$ CBO9780511546945.

[118] S. Bohn, L. Pauchard, and Y. Couder. "Hierarchical crack pattern as formed by successive domain divisions." Physical Review E 71.4 (2005), p. 046214. ISSN: 1539-3755. DOI: 10 . 1103/PhysRevE. 71.046214.

[119] P. M. Donnelly, D. Bonetta, H. Tsukaya, R. E. Dengler, and N. G. Dengler. “Cell Cycling and Cell Enlargement in Developing Leaves of Arabidopsis". Developmental Biology 215.2 (1999), pp. 407-419. ISSN: 00121606. DOI: $10.1006 /$ dbio.1999. 9443.

[120] J. Kang, Y. Mizukami, H. Wang, L. Fowke, and N. G. Dengler. "Modification of cell proliferation patterns alters leaf vein architecture in Arabidopsis thaliana". Planta 226.5 (2007), pp. 1207-1218. ISSN: 0032-0935. DOI: 10.1007/s00425-007-0567-2.

[121] S. Dhondt et al. "Quantitative analysis of venation patterns of Arabidopsis leaves by supervised image analysis". The Plant Journal 69.3 (2012), pp. 553-563. ISSN: 09607412. DOI: $10.1111 / j .1365-313 x .2011 .04803 . x$.

[122] T. J. Brodribb, T. S. Feild, and G. J. Jordan. "Leaf Maximum Photosynthetic Rate and Venation Are Linked by Hydraulics". Plant Physiology 144.4 (2007), pp. 1890-1898. ISSN: 0032-0889. DOI: $10.1104 / \mathrm{pp} .107 .101352$.

[123] C. K. Boyce, T. J. Brodribb, T. S. Feild, and M. A. Zwieniecki. "Angiosperm leaf vein evolution was physiologically and environmentally transformative". Proceedings of the Royal Society B: Biological Sciences 276.1663 (2009), pp. 1771-1776. ISSN: 0962-8452. DOI: 10.1098/rspb.2008.1919.

[124] T. J. Brodribb and T. S. Feild. "Leaf hydraulic evolution led a surge in leaf photosynthetic capacity during early angiosperm diversification". Ecology Letters 13.2 (2010), pp. 175-183. ISSN: 1461023X. DOI: $10.1111 / j .1461-0248.2009 .01410 . x$. 
[125] D. J. Peppe et al. "Sensitivity of leaf size and shape to climate: global patterns and paleoclimatic applications". New Phytologist 190.3 (2011), pp. 724-739. ISSN: 0028646X. DOI: $10.1111 / j .1469-8137.2010 .03615 . x$.

[126] D. Uhl and V. Mosbrugger. "Leaf venation density as a climate and environmental proxy: a critical review and new data". Palaeogeography, Palaeoclimatology, Palaeoecology 149.1-4 (1999), pp. 15-26. ISSN: 00310182. DOI: 10.1016/S0031-0182 (98) 00189-8.

[127] P. Sawangchote, P. J. Grote, and D. L. Dilcher. "Tertiary leaf fossils of Mangifera (Anacardiaceae) from Li Basin, Thailand as examples of the utility of leaf marginal venation characters". American Journal of Botany 96.11 (2009), pp. 2048-2061. ISSN: 0002-9122. DOI: $10.3732 / \mathrm{ajb} .0900086$.

[128] H. Ronellenfitsch, J. Lasser, D. C. Daly, and E. Katifori. “Topological phenotypes constitute a new dimension in the phenotypic space of leaf venation networks". PLoS Computational Biology 11.12 (2015), e1004680. DOI: 10.1371 / journal . pcbi. 1004680 . arXiv: 1507. 04487.

[129] R. W. H. Verwer, J Van Pelt, and H. B. M. Uylings. "An introduction to topological analysis of neurones". In: Quantitative Methods in Neuroanatomy. Ed. by M. G. Steward. John Wiley \& Sons, 1992. Chap. 12, pp. 295-323.

[130] J. Van Pelt, H. B. M. Uylings, and R. W. H. Verwer. “Tree asymmetry - A sensitive and practical measure for binary topological trees". Bulletin of Mathematical Biology 54.5 (1992), pp. 759-784. ISSN: 00928240. DOI: $10.1016 /$ S0092-8240 (05) 80142-9.

[131] H. Uylings and J. van Pelt. "Measures for quantifying dendritic arborizations". Network: Computation in Neural Systems 13.3 (2002), pp. 397-414. ISSN: 0954-898X. DOI: $10.1088 /$ 0954-898X/13/3/309.

[132] S. Boyd and L. Vandenberghe. Convex Optimization. Cambridge University Press, 2004. ISBN: 978-0-521-83378-3.

[133] R. W. Verwer and J. van Pelt. "Descriptive and comparative analysis of geometrical properties of neuronal tree structures". Journal of Neuroscience Methods 18.1-2 (1986), pp. 179-206. ISSN: 01650270. DOI: $10.1016 / 0165-0270$ (86) 90119-6.

[134] R. W. Verwer and J. van Pelt. "A new method for the topological analysis of neuronal tree structures". Journal of Neuroscience Methods 8.4 (1983), pp. 335-351. ISSN: 01650270. DOI: 10.1016/0165-0270(83) 90091-2.

[135] R. E. Horton. "Erosional development of streams and their drainage basins; hydrophysical approach to quantitative morphology". Geological Society of America Bulletin 56.3 (1945), p. 275. ISSN: 0016-7606. DOI: $10.1130 / 0016-7606$ (1945) 56 [275:EDOSAT] 2.0.CO; 2.

[136] A. N. Strahler. "Dynamic Basis of Geomorphology". Geological Society of America Bulletin 63.9 (1952), p. 923. ISSN: 0016-7606. DOI: $10.1130 / 0016-7606$ (1952) 63 [ 923 :DBOG] $2.0 . \mathrm{CO} ; 2$.

[137] R. L. Shreve. "Statistical Law of Stream Numbers". The Journal of Geology 74.1 (1966), pp. 17-37. ISSN: 0022-1376. DOI: $10.1086 / 627137$.

[138] J. W. Kirchner. "Statistical inevitability of Horton's laws and the apparent randomness of stream channel networks". Geology 21.7 (1993), pp. 591-594. ISSN: 0091-7613. DOI: 10.1130/0091-7613(1993)021<0591: SIOHSL>2.3.CO; 2 .

[139] R. Albert and A.-L. Barabási. "Statistical mechanics of complex networks". Reviews of Modern Physics 74.1 (2002), pp. 47-97. DOI: 10.1103/RevModPhys. 74 . 47. 
Bibliography

[140] R. Louf and M. Barthelemy. "A typology of street patterns". Journal of The Royal Society Interface 11.101 (2014), p. 20140924. ISSN: 1742-5689. DOI: 10.1098/rsif. 2014.0924.

[141] L. J. Billera, S. P. Holmes, and K. Vogtmann. "Geometry of the Space of Phylogenetic Trees". Advances in Applied Mathematics 27.4 (2001), pp. 733-767. ISSN: 01968858. DOI: $10.1006 /$ aama. 2001.0759.

[142] P. Ferraro and C. Godin. "A distance measure between plant architectures". Annals of Forest Science 57.5 (2000), pp. 445-461. ISSN: 1286-4560. DOI: 10 . 1051 / forest : 2000134.

[143] P. Ferraro, L. Tichit, and S. Dulucq. "Local similarity between trees". In: Acte de JOBIM '04. 2004.

[144] B. S. Everitt, S. Landau, M. Leese, and D. Stahl. Cluster Analysis. 5th ed. Vol. 62. Wiley Series in Probability and Statistics 3. Chichester, UK: John Wiley \& Sons, Ltd, 2011, pp. 202202. ISBN: 9780470977811. DOI: $10.1002 / 9780470977811$.

[145] A. Weeks, D. C. Daly, and B. B. Simpson. "The phylogenetic history and biogeography of the frankincense and myrrh family (Burseraceae) based on nuclear and chloroplast sequence data". Molecular Phylogenetics and Evolution 35.1 (2005), pp. 85-101. ISSN: 10557903. DOI: $10.1016 / j \cdot y$ mpev.2004.12.021.

[146] D. C. d. B. Daly, P. V. A. Fine, and M. C. Martínez-Habibe. "Burseraceae: a model for studying the Amazon flora". Rodriguésia 63.1 (2012), pp. 021-030. ISSN: 2175-7860. DOI: $10.1590 / \mathrm{S} 2175-78602012000100002$.

[147] T. Nelson. "Leaf Vascular Pattern Formation". The Plant Cell 9.7 (1997), pp. 1121-1135. ISSN: 10404651. DOI: 10.1105/tpc.9.7.1121.

[148] A. Perna, P. Kuntz, and S. Douady. "Characterization of spatial networklike patterns from junction geometry". Physical Review E 83.6 (2011), p. 066106. ISSN: 1539-3755. DOI: 10.1103/PhysRevE.83.066106.

[149] H. Byrne, J. King, D. McElwain, and L. Preziosi. "A two-phase model of solid tumour growth". Applied Mathematics Letters 16.4 (2003), pp. 567-573. ISSN: 08939659. DOI: 10. 1016/S0893-9659(03)00038-7.

[150] T. Bittig, O. Wartlick, A. Kicheva, M. González-Gaitán, and F. Jülicher. “Dynamics of anisotropic tissue growth". New Journal of Physics 10.6 (2008), p. 063001. ISSN: 1367-2630. DOI: $10.1088 / 1367-2630 / 10 / 6 / 063001$.

[151] A. Vasco, M. Thadeo, M. Conover, and D. C. Daly. "Preparation of Samples for Leaf Architecture Studies, A Method for Mounting Cleared Leaves". Applications in Plant Sciences 2.9 (2014), p. 1400038. ISSN: 2168-0450. DOI: 10.3732 / apps . 1400038.

[152] J. Lasser and E. Katifori. “NET: A new framework for the vectorization and examination of network data". In preparation (2015).

[153] C. A. Price, O. Symonova, Y. Mileyko, T. Hilley, and J. S. Weitz. "Leaf Extraction and Analysis Framework Graphical User Interface: Segmenting and Analyzing the Structure of Leaf Veins and Areoles". Plant Physiology 155.1 (2011), pp. 236-245. ISSN: 0032-0889. DOI: $10.1104 / \mathrm{pp} .110 .162834$.

[154] C. A. Price, P. R. T. Munro, and J. S. Weitz. “Estimates of Leaf Vein Density Are Scale Dependent". Plant Physiology 164.1 (2014), pp. 173-180. ISSN: 0032-0889. DOI: 10.1104 / pp.113.224451. 
[155] L. Sack et al. "Leaf Vein Length per Unit Area Is Not Intrinsically Dependent on Image Magnification: Avoiding Measurement Artifacts for Accuracy and Precision". Plant Physiology 166.2 (2014), pp. 829-838. ISSN: 0032-0889. DOI: 10.1104 /pp. 114.237503.

[156] C. D. Modes, M. O. Magnasco, and E. Katifori. "Extracting Hidden Hierarchies in 3D Distribution Networks". To appear (2014). arXiv: 1410.3951.

[157] N. M. Holbrook and M. A. Zwieniecki. Vascular Transport in Plants. Physiological Ecology. Academic Press, 2004. ISBN: 978-0120884575.

[158] G. A. Thompson and A. J. E. van Bel. Phloem: Molecular Cell Biology, Systemic Communication, Biotic Interactions. Ed. by G. A. Thompson and A. J. E. van Bel. Oxford, UK: Wiley-Blackwell, 2012. ISBN: 9781118382806. DOI: $10.1002 / 9781118382806$.

[159] S. Wright. The roles of mutation, inbreeding, crossbreeding and selection in evolution. 1932.

[160] H. Ronellenfitsch, M. Timme, and D. Witthaut. "A Dual Method for Computing Power Transfer Distribution Factors". Under review at IEEE Transactions on Power Systems (2015). arXiv: 1510.04645.

[161] J. Kaplan. "The end of the adaptive landscape metaphor?" Biology \& Philosophy 23.5 (2008), pp. 625-638. ISSN: 0169-3867. DOI: $10.1007 / \mathrm{s} 10539-008-9116-z$.

[162] D. M. McCandlish. “Visualizing fitness landscapes". Evolution 65.6 (2011), pp. 1544-1558. ISSN: 00143820. DOI: $10.1111 / j .1558-5646.2011 .01236 . x$.

[163] L. Horwitz. "Some Simplified Mathematical Treatments of Translocation in Plants." Plant Physiology 33.2 (1958), pp. 81-93. ISSN: 0032-0889. DOI: 10.1104 /pp.33.2 .81.

[164] M. V. Thompson and N. M. Holbrook. "Application of a Single-solute Non-steady-state Phloem Model to the Study of Long-distance Assimilate Transport". Journal of Theoretical Biology 220.4 (2003), pp. 419-455. ISSN: 00225193. DOI: 10.1006 / jtbi.2003.3115.

[165] T. Y. Cath, A. E. Childress, and M. Elimelech. "Forward osmosis: Principles, applications, and recent developments". Journal of Membrane Science 281.1-2 (2006), pp. 70-87. ISSN: 03767388. DOI: $10.1016 / \mathrm{j}$.memsci.2006.05.048.

[166] S. H. Russell and R. F. Evert. "Leaf vasculature in Zea mays L." Planta 164.4 (1985), pp. 448-458. ISSN: 0032-0935. DOI: $10.1007 / \mathrm{BF} 00395960$.

[167] J. M. Dannenhoffer, W. Ebert, and R. F. Evert. "Leaf Vasculature in Barley, Hordeum vulgare (Poaceae)". American Journal of Botany 77.5 (1990), p. 636. ISSN: 00029122. DOI: $10.2307 / 2444810$.

[168] J. T. Colbert and R. F. Evert. "Leaf vasculature in sugarcane (Saccharum officinarum L.)" Planta 156.2 (1982), pp. 136-151. ISSN: 0032-0935. DOI: 10.1007 /BF 00395428.

[169] S. T. Drobnitch, K. H. Jensen, P. Prentice, and J. Pittermann. “Convergent evolution of vascular optimization in kelp (Laminariales)". Proceedings of the Royal Society B: Biological Sciences 282.1816 (2015), p. 20151667. ISSN: 0962-8452. DOI: 10.1098 / rspb. 2015.1667.

[170] A. J. Wood, B. F. Wollenberg, and G. B. Sheblé. Power Generation, Operation, and Control. Third. John Wiley \& Sons, 2014. ISBN: 9781118585955.

[171] G. A. Pagani and M. Aiello. "The Power Grid as a complex network: A survey". Physica A: Statistical Mechanics and its Applications 392.11 (2013), pp. 2688-2700. ISSN: 03784371. DOI: $10.1016 / j$.physa.2013.01.023.arXiv: 1105.3338.

[172] M. Bernot, V. Caselles, and J.-M. Morel. Optimal Transportation Networks. Vol. 1955. Lecture Notes in Mathematics 1955. Berlin, Heidelberg: Springer Berlin Heidelberg, 2009. ISBN: 978-3-540-69314-7. DOI: 10 . 1007 / 978-3-54 0-69315-4. 
Bibliography

[173] D. A. Coomes, S. Heathcote, E. R. Godfrey, J. J. Shepherd, and L. Sack. "Scaling of xylem vessels and veins within the leaves of oak species". Biology Letters 4.3 (2008), pp. 302-306. ISSN: 1744-9561. DOI: 10.1098/rsbl.2008.0094.

[174] T. J. Brodribb. "Xylem hydraulic physiology: The functional backbone of terrestrial plant productivity". Plant Science 177.4 (2009), pp. 245-251. ISSN: 01689452. DOI: 10.1016 / j . plantsci.2009.06.001.

[175] J. S. Sperry and J. E. M. Sullivan. "Xylem Embolism in Response to Freeze-Thaw Cycles and Water Stress in Ring-Porous, Diffuse-Porous, and Conifer Species". Plant Physiology 100.2 (1992), pp. 605-613. ISSN: 0032-0889. DOI: $10.1104 /$ pp .100 .2.605.

[176] J. Pittermann and J. Sperry. "Tracheid diameter is the key trait determining the extent of freezing-induced embolism in conifers". Tree Physiology 23.13 (2003), pp. 907-914. ISSN: 0829-318X. DOI: 10.1093/treephys/23.13.907.

[177] L. J. Hickey and J. A. Doyle. "Early cretaceous fossil evidence for angiosperm evolution". The Botanical Review 43.1 (1977), pp. 3-104. ISSN: 0006-8101. DOI: 10.1007 /BF 02860849.

[178] R. S. Smith and E. M. Bayer. "Auxin transport-feedback models of patterning in plants". Plant, Cell E Environment 32.9 (2009), pp. 1258-1271. ISSN: 01407791. DOI: 10.1111 / j . $1365-3040.2009 .01997 . x$.

[179] M. G. Sawchuk and E. Scarpella. "Polarity, Continuity, and Alignment in Plant Vascular Strands". Journal of Integrative Plant Biology 55.9 (2013), pp. 824-834. ISSN: 16729072. DOI: $10.1111 /$ jipb.12086.

[180] A. Runions et al. "Modeling and visualization of leaf venation patterns". ACM Transactions on Graphics 24.3 (2005), p. 702. ISSN: 07300301. DOI: $10.1145 / 1073204.1073251$.

[181] D. Hu and D. Cai. "Adaptation and Optimization of Biological Transport Networks". Physical Review Letters 111.13 (2013), p. 138701. ISSN: 0031-9007. DOI: 10 . 1103 / Phys RevLett. 111.138701.

[182] K. Sinclair and R. C. Ball. "Mechanism for Global Optimization of River Networks from Local Erosion Rules". Physical Review Letters 76.18 (1996), pp. 3360-3363. ISSN: 0031-9007. DOI: $10.1103 /$ PhysRevLett. 76.3360.

[183] D. Barber. Bayesian Reasoning and Machine Learning. Cambridge: Cambridge University Press, 2011, p. 646. ISBN: 9780521518147 . DOI: $10.1017 /$ CBO 9780511804779.

[184] M. Wernick, Y. Yang, J. Brankov, G. Yourganov, and S. Strother. "Machine Learning in Medical Imaging". IEEE Signal Processing Magazine 27.4 (2010), pp. 25-38. ISSN: 1053-5888. DOI: 10.1109 /MSP . 2010.936730.

[185] J. H. Friedman. "Data Mining and Statistics: What's the Connection?" In: 29th Symposium on the Interface. Ed. by D Scott. 1998.

[186] S. J. Russel and P. Norvig. Artificial Intelligence: A Modern Approach. 3rd ed. Prentice Hall, 2009.

[187] F. Pedregosa et al. "Scikit-learn: Machine learning in Python". Journal of Machine Learning Research 12 (2011), pp. 2825-2830.

[188] G. Casella and R. L. Berger. Statistical Inference. 2nd ed. Duxbury Press, 2001. ISBN: 9780534243128.

[189] C. E. Shannon. "A mathematical theory of communication". ACM SIGMOBILE Mobile Computing and Communications Review 5.1 (2001), p. 3. ISSN: 15591662. DOI: 10.1145 / 584091.584093. 
[190] S. Kullback and R. A. Leibler. "On Information and Sufficiency". The Annals of Mathematical Statistics 22.1 (1951), pp. 79-86. ISSN: 0003-4851. DOI: 10.1214/aoms/1177729694.

[191] A. Kraskov, H. Stögbauer, R. G. Andrzejak, and P. Grassberger. "Hierarchical Clustering Based on Mutual Information" (2003), pp. 1-11. arXiv: q-bio/ 0311039.

[192] J. Lin. "Divergence measures based on the Shannon entropy". IEEE Transactions on Information Theory 37.1 (1991), pp. 145-151. ISSN: 00189448. DOI: $10.1109 / 18.61115$.

[193] D. Endres and J. Schindelin. "A new metric for probability distributions". IEEE Transactions on Information Theory 49.7 (2003), pp. 1858-1860. ISSN: 0018-9448. DOI: 10.1109 / TIT.2003.813506.

[194] H. Cramér. "On the composition of elementary errors". Skandinavisk Aktuarietidskrift 11 (1928), pp. 141-180.

[195] K. Pearson. "LIII. On lines and planes of closest fit to systems of points in space". Philosophical Magazine Series 62.11 (1901), pp. 559-572. ISSN: 1941-5982. DOI: 10 . $1080 /$ 14786440109462720 .

[196] C. Spearman. "'General Intelligence,' Objectively Determined and Measured". The American Journal of Psychology 15.2 (1904), p. 201. ISSN: 00029556. DOI: 10.2307/1412107.

[197] K. Hayashi, P. M. Bentler, and K.-H. Yuan. "On the Likelihood Ratio Test for the Number of Factors in Exploratory Factor Analysis". Structural Equation Modeling: A Multidisciplinary Journal 14.3 (2007), pp. 505-526. ISSN: 1070-5511. DOI: $10.1080 / 10705510701301891$.

[198] J. H. Ward. "Hierarchical Grouping to Optimize an Objective Function". Journal of the American Statistical Association 58.301 (1963), pp. 236-244. ISSN: 0162-1459. DOI: 10.1080 / 01621459.1963 .10500845$.

[199] R. A. Fisher. "The use of multiple measurements in taxonomic problems". Annals of Eugenics 7.2 (1936), pp. 179-188. ISSN: 20501420. DOI: 10.1111 / j.1469-1809.1936. tb02137.x.

[200] R. O. Duda, P. E. Hart, and D. G. Stork. Pattern Classification. 2nd ed. New York: John Wiley \& Sons, 2000. ISBN: 978-0-471-05669-0.

[201] N. Otsu. "A Threshold Selection Method from Gray-Level Histograms". IEEE Transactions on Systems, Man, and Cybernetics 9.1 (1979), pp. 62-66. ISSN: 0018-9472. DOI: 10.1109 / TSMC. 1979.4310076.

[202] K.-C. Fan, D.-F. Chen, and M.-G. Wen. "A new vectorization-based approach to the skeletonization of binary images". In: Proceedings of 3rd International Conference on Document Analysis and Recognition. Vol. 2. IEEE Comput. Soc. Press, 1995, pp. 627-630. ISBN: 0-8186-7128-9. DOI: 10.1109/ICDAR.1995.601974.

[203] J. J. Zou, H.-H. Chang, and H. Yan. "A new skeletonization algorithm based on constrained Delaunay triangulation". In: ISSPA '99. Proceedings of the Fifth International Symposium on Signal Processing and its Applications (IEEE Cat. No.99EX359). Vol. 2. Queensland Univ. Technol, 1999, pp. 927-930. ISBN: 1-86435-451-8. DOI: 10.1109 / ISSPA. 1999.815823.

[204] C.-H. Teh and R. Chin. "On the detection of dominant points on digital curves". IEEE Transactions on Pattern Analysis and Machine Intelligence 11.8 (1989), pp. 859-872. ISSN: 01628828. DOI: $10.1109 / 34.31447$.

[205] L. P. Chew. "Constrained Delaunay triangulations". In: Proceedings of the third annual symposium on Computational geometry - SCG '87. Vol. 4. New York, New York, USA: ACM Press, 1987, pp. 215-222. ISBN: 0897912314. DOI: 10.1145/41958. 41981. 
Bibliography

[206] P.-E. Danielsson. "Euclidean distance mapping". Computer Graphics and Image Processing 14.3 (1980), pp. 227-248. ISSN: 0146664X. DOI: $10.1016 / 0146-664$ X (80 ) 90054-4.

[207] A. Das, A. Bucksch, C. A. Price, and J. S. Weitz. "ClearedLeavesDB: an online database of cleared plant leaf images". Plant Methods 10.1 (2014), p. 8. ISSN: 1746-4811. DOI: 10 . 1186/1746-4811-10-8.

[208] K. Paton. "An algorithm for finding a fundamental set of cycles of a graph". Communications of the ACM 12.9 (1969), pp. 514-518. ISSN: 00010782. DOI: 10 . $1145 / 363219$. 363232 .

[209] L. Fiorin, T. J. Brodribb, and T. Anfodillo. "Transport efficiency through uniformity: organization of veins and stomata in angiosperm leaves". New Phytologist 209.1 (2015), pp. 216-227. ISSN: 0028646X. DOI: $10.1111 / \mathrm{nph} .13577$.

[210] R. Diestel. Graph Theory. Vol. 173. Graduate Texts in Mathematics. Berlin, Heidelberg: Springer Berlin Heidelberg, 2010. ISBN: 978-3-642-14278-9. DOI: 10 . 1007/978-3-642$14279-6$.

[211] A. Hatcher. Algebraic Topology. Cambridge University Press, 2001. ISBN: 978-0521795401.

[212] M. Polettini. "Cycle/Cocycle Oblique Projections on Oriented Graphs". Letters in Mathematical Physics 105.1 (2015), pp. 89-107. ISSN: 0377-9017. DOI: 10.1007 /s11005-014$0732-$ z. arXiv: $1405.0899 \mathrm{v} 2$.

[213] K. T. S. Oldham. "The doctrine of description: Gustav Kirchhoff, classical physics, and the "purpose of all science" in 19th-century Germany". PhD thesis. University of California, Berkeley, 2008. ISBN: ISBN 0-549-83131-2.

[214] N. Hwang and R. Houghtalen. Fundamentals of Hydraulic Engineering Systems. Upper Saddle River, NJ: Prentice Hall, 1996. ISBN: 9780195107326.

[215] A. J. Osiadacz. Simulation and analysis of gas networks. E. \& F. N. Spon Ltd, 1987. ISBN: 0-419-12480-2.

[216] M. Schindler and A. Ajdari. "Droplet Traffic in Microfluidic Networks: A Simple Model for Understanding and Designing". Physical Review Letters 100.4 (2008), p. 044501. ISSN: 0031-9007. DOI: 10.1103/PhysRevLett.100.044501.

[217] D. A. Sessoms et al. "Droplet motion in microfluidic networks: Hydrodynamic interactions and pressure-drop measurements". Physical Review E 80.1 (2009), p. 016317. ISSN: 15393755. DOI: $10.1103 /$ PhysRevE. 80.016317.

[218] W. H. Press, S. Teukolsky, W. T. Vetterling, and B. P. Flannery. Numerical recipes 3rd edition: The art of scientific computing. Cambridge University Press, 2007. ISBN: 9780521880688.

[219] C. D. Meyer, Jr. "Generalized Inversion of Modified Matrices". SIAM Journal on Applied Mathematics 24.3 (1973), pp. 315-323. ISSN: 0036-1399. DOI: 10.1137/ 0124033.

[220] W. Xiao and I. Gutman. "Resistance distance and Laplacian spectrum". Theoretical Chemistry Accounts: Theory, Computation, and Modeling (Theoretica Chimica Acta) 110.4 (2003), pp. 284-289. ISSN: 1432-881X. DOI: 10.1007/s00214-003-0460-4.

[221] I. Gutman and W. Xiao. "Generalized inverse of the Laplacian matrix and some applications". Bulletin: Classe des sciences mathématiques et naturelles 129.29 (2004), pp. 15-23. ISSN: 0561-7332. DOI: 10.2298 /BMAT $0429015 \mathrm{G}$.

[222] S. Redner. "Fractal and Multifractal Scaling of Electrical Conduction in Random Resistor Networks". In: Encyclopedia of Complexity and Systems Science. Ed. by R. A. Meyers. New York, NY: Springer New York, 2009, pp. 3737-3754. ISBN: 978-0-387-75888-6. DOI: 10 . 1007/978-0-387-30440-3_220. arXiv:0710.1105. 
[223] H. H. Winter. C7.4 Viscous Dissipation Term in Energy Equation. Vol. Series C. Volume 7. American Institute of Chemical Engineers, 1987, pp. 27-34. ISBN: 0-8169-0415-4.

[224] R. M. Cohn. "The resistance of an electrical network". Proceedings of the American Mathematical Society 1.3 (1950), pp. 316-316. ISSN: 0002-9939. DOI: 10 . 1090 / S0 $002-9939-$ $1950-0036696-4$.

[225] L. Ahlfors. Complex Analysis. 3rd ed. McGraw-Hill, 1979. ISBN: 978-0070006577.

[226] W. Duke and Ö. Imamoğlu. "The zeros of the Weierstrass $\wp$-function and hypergeometric series". Mathematische Annalen 340.4 (2008), pp. 897-905. ISSN: 0025-5831. DOI: $10.1007 /$ s00208-007-0174-3. 



\title{
Curriculum vitæ
}

\section{personal}

\author{
name Henrik Michael Ronellenfitsch \\ born 19 August 1987 in Homburg, Germany \\ nationality German \\ e-mail henrik.ronellenfitsch@gmail.com
}

\section{education and academia}

10/2012-present PhD student, Max Planck Institute for Dynamics and SelfOrganization, Göttingen,

Physics of Biological Organization, supervised by Eleni Katifori

03/2012-06/2012 Research assistant, ETH Zürich, Quantum Field Theory and Strings (Gaberdiel)

10/2010-03/2012 ETH Zürich, MSc Physics ETH "with distinction," thesis "Twisted Twining Genera in Mathieu Moonshine" supervised by Matthias R. Gaberdiel

09/2007-10/2010 ETH Zürich, BSc Physics ETH

\section{awards, scholarships, grants}

04/2013 Max Planck Excellence Fellowship (2+1 years), IMPRS Physics of Biological and Complex Systems, Göttingen

$03 / 2012$ Willi Studer Prize for best ETH Master's diploma in Physics

2013, 2014, 2015 IMPRS Physics of Biological and Complex Systems Göttingen Travel Grant 


\section{teaching}

2014 supervision of Bachelor's thesis "Investigation of optimisation models for the vascular network of dicotyledonous plant leaves" by Stephan Monecke, University of Göttingen

2014 co-supervision of Bachelor's thesis "Measuring the conductivity profile of a leaf vascular network" by Torsten Eckstein, University of Göttingen

2013 teaching assistant "Principles of Self-Organization in Biophysics," "Mathematical Methods of Physics," University of Göttingen

2010-2012 teaching assistant for undergraduate mathematics (Linear Algebra, Numerical Methods, MATLAB), ETH Zürich

\section{skills}

natural languages

programming languages

computing
German (native), English (fluent), French, Russian (basic knowledge)

Python, Mathematica, MATLAB (good knowledge); $C$ (basic knowledge)

Oracle Grid Engine, git (basic knowledge)

\section{list of publications}

H. Ronellenfitsch and E. Katifori. "Stochasticity, growth, and the emergence of loops in auxin canalization models". In preparation (2015)

H. Ronellenfitsch, D. Manik, and D. Witthaut. "Dual theory of transmission line outages". In preparation (2015)

T. Eckstein, H. Ronellenfitsch, J. Lasser, and E. Katifori. "Scaling of xylem vessels, sap flow speed and hydraulic conductivity of leaves". In preparation (2015)

H. Ronellenfitsch, M. Timme, and D. Witthaut. "A Dual Method for Computing Power Transfer Distribution Factors". Under review at IEEE Transactions on Power Systems (2015). arXiv: 1510.04645

H. Ronellenfitsch, J. Lasser, D. C. Daly, and E. Katifori. “Topological phenotypes constitute a new dimension in the phenotypic space of leaf venation networks". PLoS Computational Biology 11.12 (2015), e1004680. DOI: $10.1371 /$ journal.pcbi.1004680. arXiv: 1507.04487 
H. Ronellenfitsch, J. Liesche, K. H. Jensen, N. M. Holbrook, A. Schulz, and E. Katifori. "Scaling of phloem structure and optimality of photoassimilate transport in conifer needles". Proceedings of the Royal Society B: Biological Sciences 282.1801 (2015), p. 20141863. ISSN: 0962-8452. DOI: $10.1098 / \mathrm{rspb} .2014 .1863$. arXiv: 1412.1272

M. R. Gaberdiel, D. Persson, H. Ronellenfitsch, and R. Volpato. “Generalized Mathieu Moonshine". Communications in Number Theory and Physics 07 (2013), pp. 145-223. DOI: $10.4310 /$ CNTP.2013.v7.n1.a5. arXiv: 1211.7074

\section{contributed talks and posters}

APS March Meeting 2013 "Optimal Nutrient Uptake in Biological Transport Networks"

Dynamics Days Europe 2013 "Physical properties of the phloem constrain size and shape of leaves"

DPG Spring Meeting 2014 "Structure and Topology of Optimal Transport Networks in Plant Leaves"

APS March Meeting 2015 "Topological Phenotypes in Leaf Vascular Networks"

DPG Spring Meeting 2015

"Topological Phenotypes in Leaf Vascular Networks" (poster) 